\title{
Guidelines for the Treatment of Pulmonary Hypertension (JCS 2017/JPCPHS 2017)
}

Keiichi Fukuda; Hiroshi Date; Shozaburo Doi; Yoshihiro Fukumoto; Norihide Fukushima;

Masaru Hatano; Hiroshi Ito; Masataka Kuwana; Hiromi Matsubara; Shin-ichi Momomura;

Masaharu Nishimura; Hitoshi Ogino; Toru Satoh; Hiroaki Shimokawa; Keiko Yamauchi-Takihara;

Koichiro Tatsumi; Hatsue Ishibashi-Ueda; Norikazu Yamada; Shunji Yoshida; Kohtaro Abe;

Aiko Ogawa; Takeshi Ogo; Takatoshi Kasai; Masaharu Kataoka; Takashi Kawakami;

Shigetoyo Kogaki; Mashio Nakamura; Tomotaka Nakayama; Mari Nishizaki; Koichiro Sugimura;

Nobuhiro Tanabe; Ichizo Tsujino; Atsushi Yao; Takashi Akasaka; Motomi Ando; Takeshi Kimura;

Takayuki Kuriyama; Norifumi Nakanishi; Toshio Nakanishi; Hiroyuki Tsutsui

on behalf of the Japanese Circulation Society and the Japanese Pulmonary Circulation and Pulmonary Hypertension Society Joint Working Group

\section{Table of Contents}

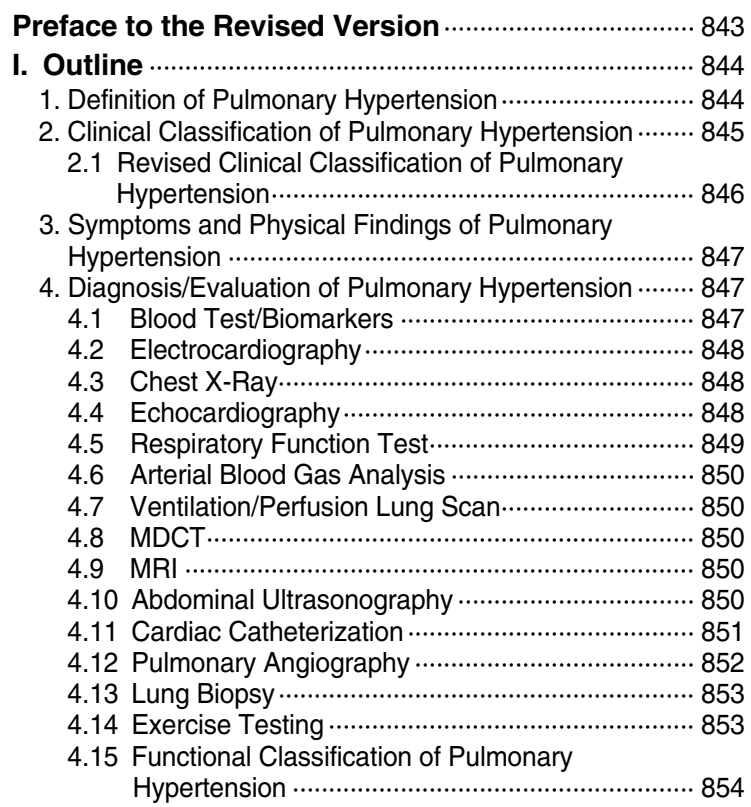

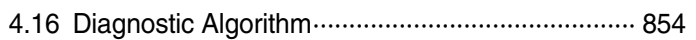

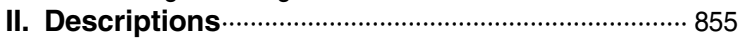

1. Pulmonary Arterial Hypertension ……............................. 855

1.1 Idiopathic Pulmonary Arterial Hypertension (IPAH)/Heritable Pulmonary Arterial Hypertension (HPAH) ……………………………… 855

1.2 Pulmonary Arterial Hypertension Associated With Connective Tissue Disease (CTD-PAH) …..... 870

1.3 Pulmonary Arterial Hypertension Associated With Portal Hypertension (PoPH) ………………... 875

1.4 Pulmonary Arterial Hypertension Associated With Adult Congenital Heart Disease ……………... 878

1.5 Drug- and Toxin-Induced Pulmonary Arterial Hypertension ………............................................. 881

1.6 PAH Associated With HIV Infection (HIV-Associated PAH) ……………………………... 881

1.7 Pulmonary Veno-Occlusive Disease (PVOD) and/or Pulmonary Capillary Hemangiomatosis $(\mathrm{PCH})$ …......................................................... 882 2. Pulmonary Hypertension Due to Left Heart Disease .... 884 2.1 Definition ……...................................................... 884

2.2 Mechanism ……............................................... 884

2.3 Epidemiology …………………………………... 885

2.4 Etiology/Diagnosis ………..................................... 885

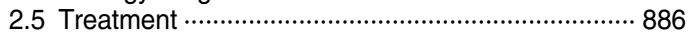

J-STAGE Advance Publication released online March 9, 2019

Mailing address: Scientific Committee of the Japanese Circulation Society, 18F Imperial Hotel Tower, 1-1-1 Uchisaiwai-cho, Chiyoda-ku, Tokyo 100-0011, Japan. E-mail: meeting@j-circ.or.jp

This English language document is a translated version of Guidelines for the Treatment of Hypertension (JCS 2017/JPCPHS 2017) reported at the Japanese Circulation Society Joint Working Groups performed in 2017. (Website: http://www.j-circ.or.jp/guideline/ pdf/JCS2017_fukuda_h.pdf).

Refer to Appendix 1 for the details of members.

The Japanese Circulation Society (JCS) and the Japanese Pulmonary Circulation and Pulmonary Hypertension Society (JPCPHS) Joint Working Group: The Japanese Circulation Society / Japanese Pulmonary Circulation and Pulmonary Hypertension Society / The Japanese Respiratory Society / Japanese College of Cardiology / The Japanese Heart Failure Society / The Japanese Society for Cardiovascular Surgery / The Japanese Association for Thoracic Surgery / The Japanese Society for Vascular Surgery / Japanese College of Angiology / The Japanese Society for Transplantation / Japan College of Rheumatology / Japanese Society of Pediatric Cardiology and Cardiac Surgery / Japanese Society for Adult Congenital Heart Disease / The Japanese Society of Pathology / Japanese Society of Phlebology / Japanese Association of Cardiovascular Intervention and Therapeutics / Japanese Society of Sleep Research / Japanese Society of Pediatric Pulmonary Circulation / Japanese Society of Vascular Pathology / The Ministry of Health, Labour and Welfare "Intractable Respiratory Disease/Pulmonary Hypertension Study Group under the Intractable and Other Disease Policy Study Program" / The Ministry of Health, Labour and Welfare "Autoimmune Disease Study Group under the Intractable Disease Policy Study Program"

ISSN-1346-9843 All rights are reserved to the Japanese Circulation Society. For permissions, please e-mail: cj@j-circ.or.jp 
3. Pulmonary Hypertension Due to Lung Diseases and/or Hypoxia ……………………………………….... 886

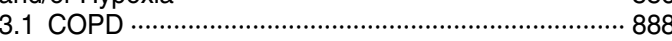

3.2 Interstitial Lung Disease ........................................... 891

3.3 Other Lung Diseases Involving Mixture of

Restrictive and Obstructive Disorders …………...... 893

3.4 Alveolar Hypoventilation Syndrome (AHS) ……...... 895

3.5 Sequelae to Pulmonary Tuberculosis....................... 895

3.6 Sleep-Disordered Breathing ……………………….. 896

4. Chronic Thromboembolic Pulmonary Hypertension

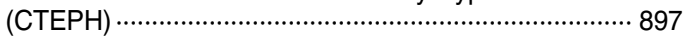

4.1 Epidemiology/Etiology ……...................................... 897

4.2 Symptoms/Clinical Findings ……............................. 899

4.3 Diagnosis and Treatment of CTEPH …………........ 899

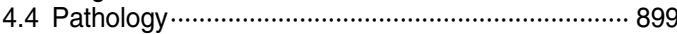

4.5 Surgical Treatment ................................................. 900

4.6 Drug Therapy and Oxygen Therapy …….................. 902

4.7 Balloon Pulmonary Angioplasty (BPA) …………..... 903
4.8 Other Diseases Requiring Distinction From CTEPH …............................................................ 904

5. Pulmonary Hypertension Due to Unclear

Multifactorial Mechanisms ……....................................... 905

6. Pulmonary Hypertension in Children ............................. 906

6.1 Idiopathic/Heritable Pulmonary Arterial Hypertension (IPAH/HPAH) …………………….. 906

6.2 Pulmonary Hypertension Due to Congenital Heart Disease …………………………………….... 910

6.3 Persistent Pulmonary Hypertension of the Newborn (PPHN) …………………………………. 915

III. Definition of Pulmonary Hypertension

Expert Referral Center ………………………...... 918

IV. Pulmonary Hypertension Under the

Measures Against Intractable Diseases ……..... 919

References ………………………................................... 926

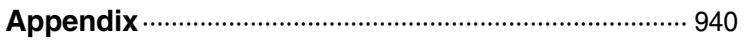

\section{Preface to the Revised Version}

Pulmonary hypertension remained an unexplained intractable disease with poor prognosis. In the last 2 decades, however, the diagnosis and treatment methods for this disease changed markedly. Depending on the location of lesions, this disease is divided into Group 1 to Group 5, and each group is subdivided according to the etiology and other factors. The genes responsible for this disease were investigated in patients with heritable pulmonary arterial hypertension. Regarding the treatment of this disease, development of prostaglandin $\mathrm{I}_{2}$ resulted in remarkable improvement of its prognosis, and subsequent introduction of endothelin receptor antagonists, and phosphodiesterase-5 inhibitors triggered further marked advances in the treatment methods for this disease. In addition, new drugs and diverse dosing methods have been developed during the past several years, making this field quite important. Regarding chronic thromboembolic pulmonary hypertension, Japanese researchers remarkably advanced balloon pulmonary angioplasty, resulting in noteworthy changes in the treatment algorithm and resulting in very favorable outcomes. Under such circumstances, a growing voice pointed out the necessity of large-scale revision of the conventional guidelines on treatment of pulmonary hypertension.

The Japanese Circulation Society, with the cooperation of the Japanese Respiratory Society, the Japan College of Rheumatology, the Japanese Association for Thoracic Surgery and other professional societies of related fields, prepared the first version of the Guidelines on Treatment of Pulmonary Hypertension in 1999 and 2000 and partially revised the Guidelines in 2006 and 2012, respectively, thus contributing to improvement of clinical management of pulmonary hypertension. Now, 5 years after the last revision, as the situations surrounding clinical management of pulmonary hypertension have changed markedly, we decided to conduct full-scale revision of the Guidelines. This time, reflecting the recent increase in the number of medical fields involved in the management of pulmonary hypertension, representatives from a very large number of professional societies and study groups participated in the revising procedure, resulting in preparation of this revised version. During the 3 sessions of the entire conference and many sessions of the group-wise conference, active and deep discussions were held by leaders of these fields in Japan. Usually, the guidelines on diagnosis and treatment of specific diseases are prepared on the basis of the stateof-art clinical evidence. In recent years, many papers have been published also in Japan, but because pulmonary hypertension is a rare disease, large-scale case registration and clinical studies are often difficult to implement in Japan. Thus, the evidence available does not seem to be sufficient in preparing guidelines unique to the Japanese situation. In Western countries, a large-scale symposium on pulmonary hypertension has been held at intervals of 5 years, and guidelines on diagnosis and treatment of this disease have begun to be prepared on the basis of the results and data from the large-scale case registration and randomized multicenter comparative studies available at a given point of time. The latest one of such guidelines is the Pulmonary Hypertension Diagnosis/Treatment Guidelines prepared by the European Society of Cardiology (ESC) and the European Respiratory Society (ERS) on the basis of the Fifth World Symposium on Pulmonary Hypertension held in Nice, France in 2013 (Nice Conference).,1,2 Our Guidelines on Treatment of Pulmonary Hypertension (revised version 2017) are fundamentally based on these latest Western guidelines, thereby incorporating much recent evidence and adding the latest treatment methods employed during clinical practice, so that these guidelines can be considerably advanced guidelines. For this reason, the statements constituting these guidelines are not always based on randomized comparative study data or objective evidence comparable to randomized comparative study data, and there are many statements that should be used only as "reference information related to treatment." These limitations need to be considered when these guidelines are utilized.

In recent years, guidelines have tended to be prepared in accordance with "Minds Handbook for Clinical Practice Guideline Development" These guidelines assumed a form consistent with the conventional guidelines prepared by the Japanese Circulation Society and adopted the "evidence levels" (Table 1) and "recommendation classes" (Table 2) based on the ESC/ERS Guidelines. The evidence levels and the recommendation classes were judged by the authors on 
the basis of the previous domestic and overseas papers and such a judgment was finally adopted by consensus among the group members and outside evaluation committee members. The revised version consists of two parts (Outline and Descriptions). Outline (Chapter I) includes explanation about the definition of pulmonary hypertension, clinical classification, symptoms/physical findings, and diagnosis methods. Descriptions (Chapter II) include explanation about the epidemiology, etiology, diagnosis methods, treatment methods, prognosis, and future perspective concerning each clinical class of pulmonary hypertension. After these two chapters, Definition of Pulmonary Hypertension Expert Referral Center (Chapter III) and Pulmonary Hypertension under the measures against intractable diseases (Chapter IV) have been added to facilitate smooth treatment of pulmonary hypertension in Japan.

Although these guidelines present standard treatment methods for pulmonary hypertension, clinical features of this disease vary among individual cases and treatment is provided under various restrictions. Therefore, treatment is not always done in accordance with these guidelines, and the statements given in the guidelines should be regarded as the "greatest common devisor" for treatment of pulmonary hypertension. The attending physician should judge a treatment method tailored to the conditions of individual cases and, if treatment is judged difficult, the patients should be referred to the hospitals affiliated with the Pulmonary Hypertension Center without delay. Furthermore, since pulmonary hypertension is an intractable orphan disease still at present, it is not easy to conduct its differential diagnosis or to select an appropriate treatment method. Treatment of this disease should be performed by or jointly with specialists having adequate experience.

Major modifications made for this version in comparison to the previous version (revised version 2012) are listed below.

1) Regarding treatment of pulmonary artery hypertension and chronic thromboembolic pulmonary hypertension, we have prepared a chart of therapeutic strategy tailored to the current status of treatment in Japan on the basis of the latest evidence and treatment alternatives.

2) "Pulmonary Hypertension in Children" (Chapter II 6.) has been set as a large section, containing all descriptions related to the pediatric field.

3) Descriptions about pathology had been made in the section on each disease in the Chapter Descriptions.

4) After the last revision of the guidelines, the number of drugs available in the treatment of pulmonary hypertension has increased. Reflecting such a change, the descriptions have been improved. Also regarding lung transplantation, the descriptions have been improved, reflecting the experience of lung transplantation in Japan accumulated after the last revision of the guidelines.

These guidelines have been prepared by cooperation of pulmonary hypertension specialists in many specialties, including not only the department of cardiology but also

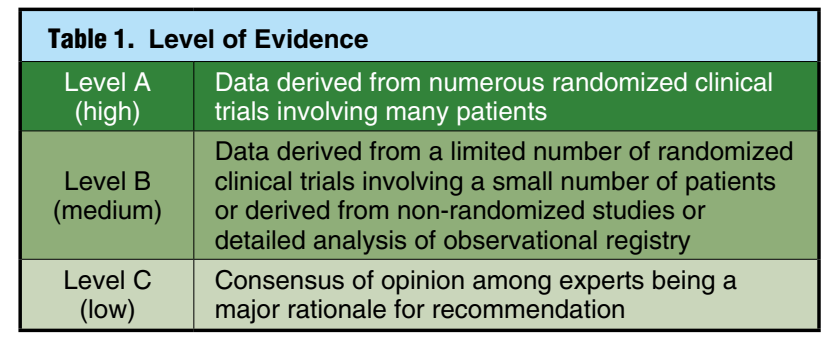

\begin{tabular}{|c|l|}
\hline \multicolumn{2}{|c|}{ Table 2. Classes of Recommendation } \\
\hline Class I & $\begin{array}{l}\text { Evidence and/or general agreement that a given } \\
\text { treatment or procedure is beneficial, useful, and } \\
\text { effective (Recommended/Indicated) }\end{array}$ \\
\hline Class II & $\begin{array}{l}\text { Conflicting evidence and/or a divergence of } \\
\text { opinion about the usefulness/efficacy of the given } \\
\text { treatment or procedure }\end{array}$ \\
\hline Class IIa & $\begin{array}{l}\text { Weight of evidence/opinion is in favor of useful- } \\
\text { ness/efficacy (Should be considered) }\end{array}$ \\
\hline Class IIb & $\begin{array}{l}\text { Usefulness/efficacy is less well established by } \\
\text { evidence/opinion (May be considered) }\end{array}$ \\
\hline Class III & $\begin{array}{l}\text { Evidence or general agreement that the given } \\
\text { treatment or procedure is not useful/effective, and } \\
\text { in some cases may be harmful (Not recommended) }\end{array}$ \\
\hline
\end{tabular}

the departments of respirology, rheumatology/collagen disease, pediatrics, cardiovascular surgery, and transplantation as well as the Ministry of Health, Labour and Welfare "Intractable Respiratory Disease/Pulmonary Hypertension Study Group under the Intractable and Other Disease Policy Study Program," and Ministry of Health, Labour and Welfare Study "Autoimmune Disease Study Group under the Intractable Disease Policy Study Program". We hope that these guidelines will be utilized by clinicians of all specialties engaged in the management of pulmonary hypertension.

During the Sixth World Symposium on Pulmonary Hypertension held in Nice, France (Nice Conference 2018), immediately before publication of these guidelines, numerous discussions were made about the diseases added or deleted to/from pulmonary hypertension although the clinical classification ( 5 groups) of this disease remained unchanged. Furthermore, during the symposium, a proposal was made to reduce the mean pulmonary artery pressure for the diagnostic criteria of pulmonary hypertension from $\geq 25 \mathrm{mmHg}$ to $\geq 20 \mathrm{mmHg}$. However, the mean pulmonary artery pressure for diagnosis of Group 4 is to be kept unchanged at $\geq 25 \mathrm{mmHg}$. Considering that no consensus has yet been reached about the proposals (including the one on the diagnostic criteria for pulmonary hypertension) made during the Nice Conference 2018, their reflection into these guidelines was avoided on the basis of consensus among the leader and all members of this committee.

\section{Outline}

\section{Definition of Pulmonary Hypertension}

In Western countries, a large-scale symposium on pulmo- nary hypertension has been held at intervals of 5 years (Table 3). During the Dana Point Conference in 2008, pulmonary hypertension was defined as mean pulmonary artery pressure (mean PAP) $\geq 25 \mathrm{mmHg}$ when measured at 


\begin{tabular}{|c|c|l|}
\hline \multicolumn{3}{|c|}{ Table 3. World Symposia on Pulmonary Hypertension } \\
\hline Year & Place & \multicolumn{1}{c|}{ Name } \\
\hline 1973 & $\begin{array}{c}\text { Geneva } \\
\text { (Switzerland) }\end{array}$ & $\begin{array}{l}\text { International Expert Conference on } \\
\text { Primary Pulmonary Hypertension }\end{array}$ \\
\hline 1998 & $\begin{array}{c}\text { Evian } \\
\text { (France) }\end{array}$ & $\begin{array}{l}\text { The Second World Symposium on } \\
\text { Pulmonary Hypertension }\end{array}$ \\
\hline 2003 & $\begin{array}{c}\text { Venice } \\
\text { (Italy) }\end{array}$ & $\begin{array}{l}\text { The Third World Symposium on } \\
\text { Pulmonary Arterial Hypertension }\end{array}$ \\
\hline 2008 & $\begin{array}{c}\text { Dana Point } \\
\text { (USA) }\end{array}$ & $\begin{array}{l}\text { The Fourth World Symposium on } \\
\text { Pulmonary Hypertension }\end{array}$ \\
\hline 2013 & $\begin{array}{c}\text { Nice } \\
\text { (France) }\end{array}$ & $\begin{array}{l}\text { The Fifth World Symposium on } \\
\text { Pulmonary Hypertension }\end{array}$ \\
\hline
\end{tabular}

rest by right heart catheterization. This definition was adopted also at the Nice Conference in 2013.1,3 According to the previous definition, mean $\mathrm{PAP} \geq 30 \mathrm{mmHg}$ at exercise was also included in pulmonary hypertension. The definition at and after the Dana Point Conference, however, did not adopt this criterion. Furthermore, among all cases of pulmonary hypertension, those presenting with pulmonary artery wedged pressure (PAWP) $\leq 15 \mathrm{mmHg}$ were defined as pulmonary arterial hypertension $(\mathrm{PAH})$ at and after the Dana Point Conference. Healthy individuals at rest have been reported to have mean PAP of $14 \pm 3 \mathrm{mmHg}$, with the upper limit of the normal range being $20 \mathrm{mmHg}{ }^{4}$ The clinical significance of cases showing mean PAP between 21 and $24 \mathrm{mmHg}$ remains to be clarified. Due to recent advances in echocardiography, it is now possible to conduct morphological evaluation of the right and left ventricles as well as estimation of the PAP and cardiac output by Doppler echocardiography. Echocardiography allows simple and noninvasive estimation of the presence of pulmonary hypertension and may be considered as a tool quite useful in the diagnosis of this disease. However, the Dana Point Conference proposed a view that direct measurement of pulmonary hemodynamics by right heart catheterization is needed at least at the time of first diagnosis or modification of treatment methods. This view now prevails as a global standard.

\section{Clinical Classification of Pulmonary Hypertension}

At the Second World Symposium on Primary Pulmonary Hypertension in 1998 co-sponsored by the World Health Organization (WHO), it was proposed to summarize cases of pulmonary hypertension resembling in etiology and pathophysiology into 5 groups (Evian Clinical Classification). This classification was later modified slightly at the Third World Symposium on Pulmonary Arterial Hypertension (PAH) held in 2003 with the support of National Institutes of Health, USA (NIH), to yield the Venice Classification.5,6 At the Fifth World Symposium on Pulmonary Hypertension in 2013 (Nice Conference), the Dana Point Classification was revised again, although slightly (reassigning neonatal persistent pulmonary hypertension to Group 1 subtype and chronic hemolytic anemia to Group 5). This classification was established in the form of Revised Clinical Classification of Pulmonary Hypertension (Dana Point Classification) at the Fourth World Symposium on Pulmonary Hypertension (Dana Point Conference) in 2008. ${ }^{7}$ To date, this classification has been adopted in
Table 4. Clinical Classification of Pulmonary Hypertension (Nice Classification [2013])

1. Pulmonary arterial hypertension

1.1 Idiopathic $\mathrm{PAH}$

1.2 Heritable $\mathrm{PAH}$

1.2.1 BMPR2

1.2.2 ALK-1, ENG, SMAD9, CAV1, KCNK3

1.2.3 Unknown

1.3 Drug and toxin induced

1.4 Associated with:

1.4.1 Connective tissue disease

1.4.2 HIV infection

1.4.3 Portal hypertension

1.4.4 Congenital heart diseases

1.4.5 Schistosomiasis

1'. Pulmonary veno-occlusive disease and/or pulmonary capillary hemangiomatosis

1". Persistent pulmonary hypertension of the newborn (PPHN)

2. Pulmonary hypertension due to left heart disease

2.1 Left ventricular systolic dysfunction

2.2 Left ventricular diastolic dysfunction

2.3 Valvular disease

2.4 Congenital/acquired left heart inflow/outflow tract obstruction and congenital cardiomyopathies

3. Pulmonary hypertension due to lung diseases and/or hypoxia

3.1 Chronic obstructive pulmonary disease

3.2 Interstitial lung disease

3.3 Other pulmonary diseases with mixed restrictive and obstructive pattern

3.4 Sleep-disordered breathing

3.5 Alveolar hypoventilation disorders

3.6 Chronic exposure to high altitude

3.7 Developmental lung diseases

4. Chronic thromboembolic pulmonary hypertension (CTEPH)

5. Pulmonary hypertension with unclear multifactorial mechanisms

5.1 Hematologic disorders: chronic hemolytic anemia, myeloproliferative disorders, splenectomy

5.2 Systemic disorders: sarcoidosis, pulmonary histiocytosis, lymphangioleiomyomatosis

5.3 Metabolic disorders: glycogen storage disease, Gaucher disease, thyroid disorders

5.4 Others: tumoral obstruction, fibrosing mediastinitis, chronic renal failure, segmental $\mathrm{PH}$

(Source: Simonneau G, et al. 20138)

the Pulmonary Hypertension Diagnosis and Treatment Guidelines prepared by the European Society of Cardiology (ESC)/European Respiratory Society (ERS) ${ }^{1,2}$ and also in the classification of pulmonary hypertension among the intractable respiratory diseases officially listed by the Japanese Ministry of Health, Labour and Welfare. Furthermore, on the basis of this classification, a large number of clinical studies have been conducted, leading to accumulation of numerous papers and findings. Because understanding of the classification is important to understand pulmonary hypertension, this revised version of the Clinical Classification of Pulmonary Hypertension (Nice Classification [2013]) (Table 4) ${ }^{8}$ is presented in detail below. 


\subsection{Revised Clinical Classification of Pulmonary Hypertension}

According to the Dana Point classification, pulmonary hypertension is divided into five groups: Group 1 (PAH), Group 2 (pulmonary hypertension due to left heart disease), Group 3 (pulmonary hypertension due to lung disease or hypoxia), Group 4 (chronic thromboembolic pulmonary hypertension; CTEPH), and Group 5 (pulmonary hypertension due to unclear multifactorial mechanisms). This basic structure of classification was maintained also in the subsequent revised version of the Clinical Classification of Pulmonary Hypertension (Nice Classification 2013).

\subsubsection{Group 1: PAH}

This is a disease group presenting with most typical clinical features of pulmonary hypertension. PAH is subdivided into idiopathic PAH (IPAH), heritable PAH (HPAH), drug- and toxin-induced PAH, PAH due to various diseases (associated PAH), pulmonary veno-occlusive disease (PVOD) and/or pulmonary capillary hemangiomatosis $(\mathrm{PCH})$, and persistent pulmonary hypertension in newborn. Associated PAH can be subdivided into connective tissue disease (CTD), human immunodeficiency virus (HIV) infection, portopulmonary hypertension $(\mathrm{PoPH})$, congenital heart disease, and schistosomiasis.

\section{a. IPAH/HPAH}

The disease concept "PAH" was first introduced at the Evian Conference in 1998. At the Venice Conference in 2003, patients with no underlying disease began to be called cases of IPAH, and patients with family history of $\mathrm{PAH}$ began to be called cases of familial PAH. At the next conference in Dana Point, "familial PAH" was renamed as "HPAH." This change of name was based on the detection in 2000 of cases of familial PAH with $B M P R 2$ gene mutation $^{9}$ as well as the detection in 2001 of patients with mutation of $A C V R L 1$ gene ( $A L K 1)$ of the transforming growth factor (TGF)- $\beta$ superfamily (identical to the family to which $B M P R 2$ gene belongs). ${ }^{10}$ At present, a diagnosis of HPAH is made in cases newly found to have gene mutation (BMPR2, ACVRL1, ENG, SMAD9) among cases clinically diagnosed as having IPAH or in cases of familial PAH according to the old classification (regardless of the presence/absence of gene mutation found). The Dana Point Conference Report is accompanied by a comment that the new category "HPAH" does not require implementation of genetic diagnosis in cases of IPAH or other cases of PAH with family history.

\section{b. Drug- and Toxin-Induced PAH}

Although the mechanism for onset of PAH remains to be clarified, cases of PAH due to some specific risk factors are known. Drugs, such as appetite suppressors, are also a risk factor. According to a report in 2006 from France, about $10 \%$ of all cases of PAH were associated with appetite suppressors, ${ }^{11}$ whereas the percentage of such cases is not high according to reports in Japan. Aminorex and fenfluramine derivatives are highly probable to trigger the onset of PAH.

\section{c. PAH Due to Connective Tissue Disease (CTD-PAH)}

The description "PAH due to collagen disease" in the Evian Classification and Venice Classification was changed to "CTD-PAH" in the Dana Point Classification. The number of patients with CTD-PAH is considered to be particularly large in Japan, making this a clinically important PAH subgroup. In Western countries, PAH due to systemic scleroderma ( $\mathrm{SSc}$ ) is predominant, with its prevalence reported to be $7-12 \% \mathbf{1 2 , 1 3}$ and with poor prognosis known in comparison to IPAH. In Japan, on the other hand, a high incidence of complication by pulmonary hypertension is found not only in cases of SSc but also in cases of mixed connective tissue disease (MCTD) or systemic lupus erythematosus (SLE). ${ }^{14}$ CTD-PAH can be characterized by the involvement of pulmonary hypertension attributable to pulmonary fibrosis, pulmonary thrombosis, or left ventricular diastolic dysfunction in addition to PAH. Because CTD-PAH cannot be considered as pure $\mathrm{PAH}$ in all cases, careful pathophysiological evaluation is needed to determine treatment methods.

\section{d. PAH Due to HIV Infection}

According to a report from France, PAH due to HIV infection accounts for about $6 \%$ of all PAH cases. ${ }^{11}$ The prevalence of PAH among patients with HIV is estimated to be about $0.5 \%,{ }^{15}$ indicating that the likelihood for HIV patients to develop PAH is not low.

\section{e. PoPH}

In foreign countries, the percentage of $\mathrm{PoPH}$ among all cases of PAH is reported to be $10 \%$ or less. ${ }^{11}$ About $5 \%$ of the patients indicated for liver transplantation have pulmonary hypertension. ${ }^{16}$ There are many unresolved questions about the mechanism for onset of PoPH. The presence of portal hypertension has been considered as a more decisive factor for onset of pulmonary hypertension than the presence of an underlying liver disease. ${ }^{17}$

\section{f. PAH Due to Congenital Heart Disease}

PAH due to congenital heart disease of systemic/pulmonary shunt type is now better understood than in the past. According to the reports from Europe and North America, the frequency of complication by PAH among patients with congenital heart disease (congenital heart disease-PAH; $\mathrm{CHD}-\mathrm{PAH}$ ) is 1.6-12.5 out of one million population, and $25-50 \%$ of the patients with this complication are estimated to lead to the severest form of CHD-PAH, i.e., Eisenmenger's syndrome. ${ }^{18}$ In patients with Eisenmenger's syndrome, right-to-left shunt is usually seen as a result of increased pulmonary vascular resistance (PVR), and the features of this disease differ from those of simple PAH.

\section{g. РAH Due to Schistosomiasis}

This is a disease added as a subgroup of PAH to the Dana Point Classification. Its similarities to IPAH in terms of clinical and pathological features have been reported. ${ }^{19}$ In countries where schistosomiasis is endemic, PAH due to schistosomiasis accounts for a high percentage of all patients with PAH, but there are few reports of this condition in Japan.

\section{h. PVOD and/or PCH}

Clinical features of these two conditions are common in many aspects with PAH, but there are some differences. In the Nice Classification (2013), PVOD and PCH were classified as a subtype of Group 1 (Group 1'). There are many histological similarities between PCH and PVOD, ${ }^{20}$ and some investigators consider them as two different phenotypes of one disease. Response to medical treatment and 
prognosis differ between $\mathrm{PAH}$ and $\mathrm{PVOD} / \mathrm{PCH}$, with the percentage of patients with poor prognosis reported to be higher among patients with $\mathrm{PVOD} / \mathrm{PCH}$.

\section{i. Persistent Pulmonary Hypertension in the Newborn}

Newborns can develop persistent hypertension, a condition called "persistent pulmonary hypertension of newborn (PPHN)." This disease was included in Group 1 (PAH) according to the Dana Point Classification, but it was regrouped as a subtype of Group 1 i.e. Group 1" in the Nice Classification (2013).

\section{2.1.2 Group 2: Pulmonary Hypertension Due to Left Heart Disease}

This was called "pulmonary hypertension with heart disease" in the Evian Classification and the Venice Classification. It was renamed as "pulmonary hypertension due to left heart disease" in the Dana Point Classification. The number of patients belonging to this group is larger than that of any other group of pulmonary hypertension. According to the etiology, this group has been divided into three subclasses: systolic dysfunction, diastolic dysfunction, and valvular disease (of the left ventricle). In the Nice Classification (2013), congenital/acquired left inflow tract/ outflow tract obstruction was added. In this group of patients, increased PAWP accompanies pulmonary hypertension, and the pulmonary vascular resistance does not show marked elevation in many cases. Thus, the pathophysiology of this group basically differs from that of PAH associated with lesions in the pulmonary vessels.

\section{2.1.3 Group 3: Pulmonary Hypertension Due to Lung Diseases and/or Hypoxia}

This group of pulmonary hypertension originates primarily of lung disease. It is accompanied by hypoxia or various lung diseases such as chronic obstructive pulmonary disease (COPD), interstitial lung disease, sleep apnea and chronic high-altitude syndrome. In cases of pulmonary hypertension due to lung parenchymal disorder, marked pulmonary hypertension has been reported to be infrequent. ${ }^{21}$

\subsubsection{Group 4: CTEPH}

$\mathrm{CTEPH}$ is a disease caused by organic thrombosis in the pulmonary artery. In Japan, it was previously called idiopathic chronic pulmonary thromboembolism (pulmonary hypertension type). At the Dana Point Classification, CTEPH was adopted as a formal term.

\section{2.1.5 Group 5: Pulmonary Hypertension Due to Unclear Multifactorial Mechanisms}

Diseases conventionally known to be complicated by pulmonary hypertension were recently incorporated into the classification. These diseases include systemic disorders (sarcoidosis), metabolic disorders (glycogen storage disease type Ia), and other conditions (tumor, dialysis). In the Nice Classification (2013), chronic hemolytic anemia, such as sickle cell anemia, thalassemia, hereditary spherocytosis, stomatocytosis, and microangiopathic hemolytic anemia, were moved from Group 1 to this group.

\section{Symptoms and Physical Findings of Pulmonary Hypertension}

The most characteristic symptom of pulmonary hyperten- sion is shortness of breath on exertion. This symptom appears early and is visible even at the stage of pulmonary vascular disease without pulmonary hypertension. Fatigue, chest pain, and syncope are also seen. Palpitation, cough, and hemoptysis are seen occasionally. If accompanied by right-sided heart failure, this condition presents with gastrointestinal symptoms, such as abdominal swelling (due to hepatic congestion or gastrointestinal edema), premature feeling of abdominal fullness, and anorexia, as well as lower leg edema.

Physical findings include accentuated pulmonary second sound, parasternal pulsation pansystolic murmur at the lower part of the left sternal edge (associated with tricuspid insufficiency; occasionally showing Rivero-Carvallo sign in which the murmur increases during inspiration), early diastolic murmur at the left sternal edge (Graham Steel murmur, associated with pulmonary valve insufficiency), and fourth right heart sound. Of these findings, accentuated pulmonary second sound and the appearance of fourth right heart sound have been reported to be seen particularly frequently. ${ }^{22}$ In the presence of right-sided heart failure, jugular vein distention, third right heart sound, hepatomegaly, lower leg edema, and ascites are observed. Physical findings associated with the diseases responsible for pulmonary hypertension include clubbed finger (seen in cases of Eisenmenger's syndrome, pulmonary veno-occlusive disease, pulmonary disease), various physical findings of each collagen disease or hepatic disease, and pulmonary artery stenotic murmur (seen in cases of CTEPH).

\section{Diagnosis/Evaluation of Pulmonary Hypertension}

\subsection{Blood Test/Biomarkers}

Pulmonary hypertension cannot be diagnosed solely on the basis of blood tests. Blood tests are conducted for evaluation of the severity and clinical course of pulmonary hypertension and diagnosis of underlying disease in patients diagnosed as having pulmonary hypertension. In mild cases of pulmonary hypertension, blood test results are often normal. As pulmonary hypertension becomes severe, resulting from loads on the right heart system or rightsided heart failure, elevation is seen in the levels of brain natriuretic peptide (BNP, N-terminal fragment of BNP precursor (NT-proBNP) and uric acid. ${ }^{23-25}$ If congestive liver develops, hepatic function becomes abnormal. Blood tests are useful in the diagnosis of underlying diseases that can lead to pulmonary hypertension. Cases of CTD-PAH require measurement of autoantibodies, inflammation markers (e.g., C-reactive protein: CRP), whereas cases of pulmonary hypertension secondary to portal hypertension require assessment of hepatic function and measurement of platelet count and bile acid level, and cases suspected of pulmonary hypertension associated with HIV require measurement of HIV antibody. ${ }^{1}$ It is not uncommon that pulmonary hypertension is complicated by thyroid disease during its course, thus requiring thyroid function tests as well. ${ }^{26}$ If chronic thromboembolic pulmonary hypertension (CTEPH) is suspected, autoantibodies involved in thrombus formation (e.g., clotting system markers, such as D-dimer, thrombus-predisposing factors, such as protein $\mathrm{C}$ or $\mathrm{S}$, lupus anticoagulants, and anti-cardiolipin antibodies) need to be examined. In patients with compromised respiratory function and patients with pulmonary hypertension sec- 


\begin{tabular}{|c|c|c|}
\hline $\begin{array}{l}\text { Peak tricuspid } \\
\text { regurgitation velocity }\end{array}$ & $\begin{array}{l}\text { Presence of other echo } \\
\text { "PH signs" }\end{array}$ & $\begin{array}{c}\text { Probability of pulmonary } \\
\text { hypertension }\end{array}$ \\
\hline$\leq 2.8 \mathrm{~m} / \mathrm{sec}$ or not measurable & No & Low \\
\hline$\leq 2.8 \mathrm{~m} / \mathrm{sec}$ or not measurable & Yes & \multirow{2}{*}{ Intermediate } \\
\hline $2.9-3.4 \mathrm{~m} / \mathrm{sec}$ & No & \\
\hline $2.9-3.4 \mathrm{~m} / \mathrm{sec}$ & Yes & \multirow{2}{*}{ High } \\
\hline$>3.4 \mathrm{~m} / \mathrm{sec}$ & Present/Absent & \\
\hline
\end{tabular}

(Source: Prepared based on Galiè N, et al. 2016')

ondary to congenital heart disease involving reverse shunt, complication by polycythemia can occur. Blood tests are needed also for the diagnosis of adverse reactions to the drugs used for treatment. Hepatic dysfunction and anemia can be induced by endothelin receptor antagonists. In cases treated with epoprostenol, platelet count can decrease, thus requiring continued measurement.

\section{| 4.2 Electrocardiography}

Electrocardiography (ECG) provides information useful in the evaluation of the severity of pulmonary hypertension. In mild cases, a normal ECG pattern is not uncommon. In severe cases, changes in ECG due to right ventricular loads appear. These changes include pulmonary $\mathrm{P}$ wave, right axis deviation, right ventricular hypertrophy (increased $R$ wave amplitude with $\mathrm{V} 1, \mathrm{R} / \mathrm{S}$ ratio $>1$ ), right ventricular strain (ST depression toward right from V1 to V3), and corrected QT (QTc) interval elongation. If right ventricular strain, QRS width enlargement, or QTc elongation are present, pulmonary hypertension may become severer. ${ }^{27,28}$ If the pulmonary artery pressure can be reduced by treatment, the load on the right ventricle/right atrium will alleviate, and this change can be used for judgment of the patient's response to treatment.

Patients with pulmonary hypertension may develop supraventricular tachycardia such as atrial flutter or fibrillation. Atrial flutter or fibrillation develops in $25 \%$ of all patients with pulmonary hypertension in 5 years. These arrhythmias reduce cardiac output triggering exacerbation of hemodynamics. In patients complaining of palpitation, the diagnosis of arrhythmia needs to be established by Holter ECG.

\subsection{Chest X-Ray}

Common findings include dilatation of the central segment of pulmonary artery, marked narrowing of the peripheral pulmonary artery, and cardiomegaly associated with right atrium/ ventricular enlargement. Abnormal chest X-ray findings include protrusion of the left second arch associated with dilatation of the main trunk of the pulmonary artery and protrusion of the left fourth arch due to right ventricular enlargement. When the right ventricular pressure increases, the right ventricular outflow tract dilates, resulting in protrusion of the left third arch. The right atrium load leads to protrusion of the right second arch. When the peripheral pulmonary arteries undergo thinning towards the tip (tapering), permeability through the peripheral lung field is increased. Pleural effusion can be detected in association with severe pulmonary hypertension. However a nor- mal chest X-ray does not exclude pulmonary hypertension.

\subsection{Echocardiography}

Echocardiography, which provides noninvasive estimation of the pulmonary artery pressure, is used as a gate keeper for the diagnosis of pulmonary hypertension. It is recommended to perform echocardiography in patients suspected of having pulmonary hypertension or who may develop pulmonary hypertension. However, a definite diagnosis of pulmonary hypertension is not possible with echocardiography alone. Right heart catheterization is indispensable to establish the diagnosis of pulmonary hypertension and to consider subsequent treatment.

Pulmonary artery systolic pressure can be estimated from the peak flow rate of tricuspid regurgitation, using simplified Bernoulli equation.

Estimated pulmonary artery systolic pressure $=$ $4 \times(\text { tricuspid regurgitation peak flow rate })^{2}+$ estimated right atrial pressure

In the past, a fixed value ( 5 or $10 \mathrm{mmHg}$ ) of estimated right atrial pressure was used for this equation. To increase the accuracy of estimated right atrial pressure, it is now recommended to estimate the right atrial pressure from the inferior vena cava diameter and its respiration-related change. Thus, if the inferior vena cava diameter is less than $21 \mathrm{~mm}$ and its diameter is reduced by $50 \%$ or more during sniffing, the estimated right atrial pressure is $3 \mathrm{mmHg}$ (normal right atrial pressure). If the inferior vena cava diameter is $21 \mathrm{~mm}$ or more and its sniffing-related change is $50 \%$ or less or its respiration-related change at rest is less than $20 \%$, the estimated right atrial pressure is $15 \mathrm{mmHg}$. In other cases falling under none of these cases, the intermediate level $(8 \mathrm{mmHg})$ is adopted as the estimated right atrial pressure. ${ }^{29}$

However, the estimated pulmonary artery pressure involves problems related to measurement of tricuspid regurgitation peak flow rate, error arising from squaring the peak flow rate, and error in estimation of the right atrial pressure. To reduce these errors, it has been recommended to use the tricuspid regurgitation peak flow rate itself for screening (Table 5). ${ }^{1}$ If this flow rate exceeds $3.4 \mathrm{~m} / \mathrm{sec}$, pulmonary hypertension is suspected. ${ }^{1}$

In cases in which tricuspid regurgitation is mild and the peak flow rate is difficult to measure, one possible solution is intravenous infusion of a contrast material to enhance the Doppler signals. There are also problems in the step of diagnosing pulmonary hypertension from the tricuspid regurgitation flow rate. The flow rate tends to be underestimated if the continuous wave Doppler beam and the 


\begin{tabular}{|c|c|}
\hline $\begin{array}{c}\text { Site } \\
\text { observed }\end{array}$ & Sign \\
\hline $\begin{array}{l}\text { Right and left } \\
\text { ventricles }\end{array}$ & $\begin{array}{l}\text { Right ventricle/left ventricle basal diameter ratio } \\
>1.0 \\
\text { Flattening of the interventricular septum } \\
\text { (particularly left ventricular eccentricity index at } \\
\text { systole }>1.1 \text { ) }\end{array}$ \\
\hline Right atrium & $\begin{array}{l}\text { End-systolic right atrium area (apical four- } \\
\text { chamber cross-section) }>18 \mathrm{~cm}^{2 *}\end{array}$ \\
\hline $\begin{array}{l}\text { Inferior vena } \\
\text { cava }\end{array}$ & $\begin{array}{l}\text { Inferior vena cava diameter }>21 \mathrm{~mm} \text { with } \\
\text { decreased inspiratory collapse }(<50 \% \text { with a } \\
\text { sniff or }<20 \% \text { with quiet inspiration) }\end{array}$ \\
\hline $\begin{array}{l}\text { Pericardial } \\
\text { effusion }\end{array}$ & Pool present \\
\hline $\begin{array}{l}\text { Pulmonary } \\
\text { artery }\end{array}$ & $\begin{array}{l}\text { Right ventricular outflow tract systolic accelera- } \\
\text { tion time }<105 \mathrm{msec} \text { or two-peak pattern } \\
\text { Early diastolic pulmonary regurgitation velocity } \\
>2.2 \mathrm{~m} / \mathrm{sec} \\
\text { Pulmonary artery diameter }>25 \mathrm{~mm}\end{array}$ \\
\hline
\end{tabular}

${ }^{*}$ Data in Western countries; possible difference in the criterion level for Japanese with smaller physiques. (Source: Prepared based on Galiè N, et al. 2016'1)

regurgitation jet are angled. In cases of severe tricuspid regurgitation, the pressure gradient related to the regurgitation flow rate is occasionally underestimated or overestimated. The echocardiographic findings following increases in pulmonary hypertension are also useful in the diagnosis. For example, the echocardiographically demonstrated right ventricular dilatation, compression of the ventricular septum by the dilated right ventricle, enlargement of the right atrium size, and pulmonary artery blood flow rate pattern are useful (Table 6). ${ }^{1}$

If a judgment of "low" is made on the basis of general assessment of these echocardiographic findings, pulmonary hypertension can be basically ruled out (Table 5). However, even in such cases, follow-up by echocardiography is recommended if the risk for CTD or CTEPH is present. In cases in which the probability of pulmonary hypertension is rated as "intermediate," detailed examination is needed about the underlying disease as the risk factor for pulmonary hypertension (e.g., collagen disease and CTEPH), and follow-up by echocardiography should be performed. For a more accurate diagnosis, it is advisable to perform additional examination tailored to individual patients, such as exercise echocardiography, and, if its results suggest pulmonary hypertension, to conduct detailed examination including right heart catheterization. Exercise echocardiography is useful in the diagnosis of early stage pulmonary hypertension or pulmonary hypertension occurring only during exertion or exercise, but there are no established criteria for such diagnosis. If the probability for pulmonary hypertension is rated as "high," right heart catheterization is recommended for a definite diagnosis.

For patients with heart disease complicated by pulmonary hypertension, echocardiography is useful also in the diagnosis of the underlying heart disease. Care is needed to avoid overlooking shunt-involving heart disease among congenital heart diseases. If pulmonary arteriovenous shunt is suspected, a definite diagnosis by multiple-detector CT (MDCT) or MRI is needed. If these noninvasive modalities do not allow a definite diagnosis of pulmonary hypertension, catheterization should be performed.
Among cases of pulmonary hypertension associated with left ventricular failure, attention is needed to the fact that cases of heart failure with preserved ejection fraction (HFpEF) have been recently increasing, and echocardiography is used for evaluation of left ventricular diastolic function. Also in such cases, right-sided cardiac catheterization is indispensable for a definite diagnosis and pathophysiological evaluation of pulmonary hypertension.

The indicators that can be evaluated by echocardiography, such as right atrial area index and indicators of right ventricular function (reduction of right ventricular function (tricuspid annular plane systolic excursion: TAPSE) and right ventricular free wall strain), are useful also as prognostic factors for pulmonary hypertension. ${ }^{30}$ Because drug therapy for pulmonary hypertension has advanced rapidly, improvement in these indicators are expected following alleviation of pulmonary hypertension by treatment even when the indicators are poor before the start of treatment. Therefore, treatment should not be discontinued even when the indicators rated by echocardiography are unfavorable. Although rarely, there are cases in which the echocardiographic indicators poorly improve despite reduction in the pulmonary artery pressure in response to treatment. Such cases need careful follow-up and management.

\section{4.5 Respiratory Function Test}

Respiratory function tests are useful in the differential diagnosis of pulmonary hypertension or ruling out other lung diseases. Among cases of pulmonary hypertension accompanied by lung disease (Group 3), respiratory function tests are useful in identification of cases in which the degree of pulmonary artery lesions is estimated to be large. In cases of mild idiopathic/congenital pulmonary arterial hypertension, spirometric parameters (vital capacity and forced expiratory volume one second percent: FEV1\%) are often normal. In the other cases of Group 1 pulmonary hypertension and all cases of Groups 2 to 5, various abnormalities are noted, ranging from restrictive disorders to obstructive disorders and from mild to severe abnormalities. In cases of combined pulmonary fibrosis and emphysema (CPFE), spirometric findings remain normal or mildly abnormal by off-setting the influence on pulmonary function between the pulmonary emphysema (of upper lung field) and interstitial lesions (of lower lung field), but frequent complication by pulmonary hypertension occurs. ${ }^{31}$

Carbon monoxide (CO) diffusing capacity (DLco) test is designed not only to determine the capacity of diffusing $\mathrm{CO}$ gas from alveoli to capillary blood in the narrower sense of the term but also to assess the process of $\mathrm{CO}$ gashemoglobin binding in the capillaries. ${ }^{32}$ This parameter depends on the magnitude of the intrapulmonary capillary bed area and blood volume. In the presence of many diseases responsible for pulmonary hypertension, the capillary blood volume decreases and DLco is thus reduced, with its level associated with poor prognosis according to a report. ${ }^{33}$ In cases of pulmonary hypertension having complicated pulmonary veno-occlusive disease (PVOD) or scleroderma and cases of pulmonary hypertension accompanied by parenchymal lung disease, DLco is often low. In cases of disease involving left-to-right shunt, on the other hand, DLco increases, reflecting the increase in pulmonary blood flow. 


\section{4.6 Arterial Blood Gas Analysis}

Because patients with pulmonary hypertension usually develop mild hypoxemia due to an abnormal ventilation/ blood flow ratio, arterial blood gas analysis is an indispensable test. Evaluation of hypoxemia is particularly important for diagnosis of Group 3 pulmonary hypertension such as chronic obstructive pulmonary disease (COPD) and interstitial pneumonia. Because hypoxemia of any type can be an aggravating factor for pulmonary hypertension mediated by pulmonary artery constriction, long-term oxygen therapy is indicated in cases in which arterial oxygen tension $\left(\mathrm{PaO}_{2}\right)$ at rest $<60 \mathrm{mmHg}$. Furthermore, since even mild hypoxemia can cause tissue hypoxia if cardiac output is reduced ${ }^{34}$ long-term oxygen therapy is covered by health insurance in Japan even when the requirement of $\mathrm{PaO}_{2}$ at rest $<60 \mathrm{mmHg}$ is not satisfied. Depending on the patient, it is necessary to evaluate hypoxemia during sleep or exercise using a pulse oximeter. In cases presenting with hypercapnia due to low alveolar ventilation, complication by pulmonary hypertension is often seen. ${ }^{35}$

\section{| 4.7 Ventilation/Perfusion Lung Scan}

A ventilation/perfusion lung scan is useful to clarify the etiology of pulmonary hypertension, particularly in diagnosing acute pulmonary thromboembolism and CTEPH. Although ventilation/perfusion lung scans are useful for detecting impaired blood flow and diagnosing the site of such disturbance, blood flow defects can occur also in lung parenchyma affected by atelectasis, pulmonary emphysema, or pneumonia. For this reason, it is recommended to conduct ventilation scan simultaneously or to use some other modalities of diagnostic imaging (chest X-ray, CT) to detect lung parenchymal lesions. In patients with stenosis or obstruction of pulmonary blood vessels (e.g., cases of CTEPH, angiitis, vasculitis), only the area of impaired blood flow is visualized as a wedged blood flow defective area, and no abnormality is visible on images other than ventilation/ perfusion lung scan. In cases of pulmonary arterial hypertension (PAH), the ventilation/perfusion lung scan is often normal, but small peripheral blood flow defects (accompanied by ventilation-blood flow mismatch) or uneven mottled patterns of blood flow are occasionally visible.

\subsection{MDCT}

Contrast-enhanced MDCT has been reported to have a diagnostic ability comparable to pulmonary angiography (PAG) also in the diagnosis of $\mathrm{CTEPH}^{36}$ and has been shown to be useful in detection of segmental thrombus or larger thrombus needed for determination of the indications for surgery according to the results of meta-analysis in comparison to ventilation/perfusion lung scans or pulmonary angiography. ${ }^{37}$ Contrast-enhanced MDCT is useful also in evaluation of the cardiovascular system in patients with pulmonary hypertension associated with congenital heart disease and in detection of pulmonary arteriovenous shunt. Thin slice MDCT without contrast material, on the other hand, allows the visualization of very small lesions in the lung field. A characteristic of CTEPH is the mosaic pattern (a pattern consisting of a mixture of ground glass opacity and low density areas). This mosaic pattern is absent in cases of $\mathrm{PAH}{ }^{38}$ In patients with pulmonary hypertension associated with lung disease, characteristic features of individual lung diseases may be observed. In patients with PVOD or pulmonary capillary hemangiomatosis $(\mathrm{PCH})$, ground glass opacity, interlobular septal thickening, and mediastinal lymph node enlargement are characteristic findings. ${ }^{39}$ The pulmonary artery diameter is used for evaluation of pulmonary hypertension. It has been reported that the inner diameter of the pulmonary artery trunk $\geq 29 \mathrm{~mm}$ (when determined on contrastenhanced CT) and its outer diameter $\geq 33 \mathrm{~mm}$ (on noncontrast CT) is useful in the diagnosis of $\mathrm{PAH}$, and that a correlation is noted between the ratio of pulmonary artery to aortic diameter and the severity of pulmonary hypertension or prognosis in patients with COPD. ${ }^{40}{ }^{42}$ In cases of pulmonary emphysema, a decrease in pulmonary small vessels can predict pulmonary hypertension and, for this reason, the percentages of total cross sectional area of small pulmonary vessels less than $5 \mathrm{~mm}^{2}(\% \mathrm{CSA}<5)$ to the total lung area have been shown to have a close inverse correlation with mean PAP. ${ }^{43}$

\subsection{MRI}

With MRI, it is possible to measure the volume of right and left ventricles (end-diastolic volume, end-systolic volume), myocardial weight of right and left ventricles, and the short-axial area of the pulmonary artery. From these parameters, stroke volume, cardiac output, right/left ventricular ejection fraction, and pulmonary artery stiffness can be calculated. These parameters are useful in judging the response of pulmonary hypertension to treatment and predicting the outcome. ${ }^{44} 47$ The visualization of pulmonary vessels by MR angiography and evaluation of lung field blood flow by perfusion MRI are useful in the diagnosis of CTEPH..$^{48}$ They are useful also in morphological evaluation of the cardiovascular system in patients with congenital heart disease.

Contrast-enhanced MRI using gadolinium occasionally reveals late gadolinium enhancement (LGE) at the right ventricle insertion points. This has been reported to correlate closely with right ventricular dilatation/hypertrophy, increased pulmonary artery pressure (PAP), and clinically aggravating events in cases of pulmonary hypertension..$^{4,50}$ Recently, new methods of myocardial strain evaluation, such as T1-mapping based right ventricular histological diagnosis, displacement encoding with stimulated echoes (DENSE), and strain encoded (SENC) method, have been developed and have begun to be performed for evaluation of right ventricular function. ${ }^{51}$

\subsection{Abdominal Ultrasonography}

Abdominal ultrasonography is used to detect complication by liver cirrhosis or portal hypertension when clarifying the etiology of pulmonary hypertension. This examination is indispensable in patients suspected of having portopulmonary hypertension $(\mathrm{PoPH})$. In cases complicated by liver cirrhosis, abdominal ultrasonography reveals morphological changes in the liver (right lobe atrophy, compensatory hypertrophy of the left lobe and caudate lobe, liver surface irregularities, and nodular changes). In cases complicated by portal hypertension, this examination reveals splenomegaly, well-developed collaterals, and ascites. Doppler abdominal ultrasonography of these cases revealed portal regurgitation and blood flow through the left gastric vein, paraumbilical vein, and splenorenal shunt. 
If right-sided heart failure due to pulmonary hypertension progression, signs of hepatic congestion (dilatation of the hepatic vein and the hepatic segment of inferior vena cava, disappearance of respiratory collapsibility) and ascites are revealed by this examination.

\subsection{Cardiac Catheterization}

Cardiac catheterization is required to confirm the diagnosis of pulmonary hypertension (PH), the classification of pulmonary hypertension, and to assess the severity of hemodynamic impairment and the therapeutic efficacy. Although the risk from right heart catheterization (RHC) in expert centers is low, it should be carefully performed especially in patients with symptoms of the New York Heart Association (NYHA)/WHO Functional Class IV. A previous study reported 76 severe complications related to RHC (1.1\%) among 7,218 sessions of RHC, including 29 events related to venous puncture, 22 events related to manipulation for RHC, 15 events related to vascular reaction test, and 6 events related to pulmonary angiography. Also, 4 fatal cases $(0.055 \%)$ have been reported, 2 cases were directly associated with RHC procedures. ${ }^{52}$ Recently, the internal jugular vein is frequently used for RHC in patients with pulmonary hypertension, in which $1.7 \%$ (6/349 sessions) of complications have been reported as follows: carotid artery puncture in 3 cases, sinus bradycardia in 2 cases, complete atrioventricular block in 1 case. ${ }^{53} \mathrm{In}$ addition, the risk from catheterization is higher in children than in adults. It has been reported that cardiopulmonary resuscitation is required in $6 \%(4 / 70)$ of the pediatric patients with pulmonary hypertension when undergoing RHC under general anesthesia. ${ }^{54}$ The threshold to perform left heart catheterization in addition to RHC varies depending on the magnitude of risk for coronary artery disease or heart failure with preserved ejection fraction (HFpEF). Especially in patients with echocardiographic signs of left ventricular systolic and/or diastolic dysfunction, left heart catheterization to measure left ventricular enddiastolic pressure is required to avoid misclassification of PH. ${ }^{55} \mathrm{It}$ is important to understand that cardiac catheterization is an invasive method for hemodynamic evaluation, and should be performed after sufficient evaluation by other non-invasive modalities (diagnostic imaging) to avoid excess risk.

The important points on performing RHC and interpretation of hemodynamic parameters, and acute pulmonary vascular reaction tests used for decision of therapeutic strategy will be described below.

\section{| 4.11.1 Right Heart Catheterization}

Zero value should be decided by setting a pressure transducer at midpoint between precordial level and bed surface level in the supine position. PAP, pulmonary arterial wedge pressure (PAWP), right ventricular pressure (RVP), and right atrial pressure (PAP) should be measured for comprehensive evaluation of hemodynamics. A balloon catheter should be used for pressure measurement during RHC, and the balloon should be inflated during advancement of the catheter. Repeated balloon deflation and inflation within pulmonary artery should be avoided, because this has been associated with rupture of pulmonary arteries.

\subsubsection{PAP}

As definition of pulmonary arterial hypertension, mean
PAP at rest $\geq 25 \mathrm{mmHg}$ has been used. ${ }^{56}$ Although the upper limit of the normal range for mean PAP is $20 \mathrm{mmHg}$, there is no sufficient evidence on the clinical significance of the PAP range from 21 to $24 \mathrm{mmHg}$. In the high risk group for PAH including family members of heritable PAH patients or those with connective tissue disease, careful follow-up is required even if their mean PAP is $21-24 \mathrm{mmHg}$. In CTEPH patients, the value of PAP can vary by the measuring sites, therefore, measuring PAP at several sites, such as bilateral main pulmonary arteries or main trunk of pulmonary artery, should be considered.

\section{411.3 PAWP}

Although the term "pulmonary capillary wedge pressure (PCWP)" had been previously used, the term pulmonary artery wedge pressure (PAWP) has been used uniformly since the Nice Conference in 2013. ${ }^{56} \mathrm{PAWP}$ is $15 \mathrm{mmHg}$ or less in patients with pre-capillary pulmonary hypertension (Groups 1, 3, 4, and 5 of the Clinical Classification of Pulmonary Hypertension), and higher than $15 \mathrm{mmHg}$ in Group 2 (pulmonary hypertension associated with left heart disease: LHD). However, in patients with pulmonary hypertension, accurate pressure measurement is sometimes difficult, because pulmonary arteries dilated by pulmonary hypertension lead to incompletely wedging into the pulmonary artery, resulting in overestimation of PAWP and abnormal pressure patterns. In these patients, accurate PAWP can be obtained with balloon size adjustment by gas volume reduction in the balloon catheter and wedging into the segmental pulmonary artery or more peripheral pulmonary artery. In CTEPH patients, it is occasionally difficult to obtain accurate PAWP at the stenotic pulmonary arterial lesions; therefore, PAWP should be measured by selecting a less severely affected vessel. It is necessary to understand that inaccurate values of PAWP lead to inappropriate clinical judgments, and make efforts to determine the accurate value of PAWP. It is recommended to measure PAWP at the end-expiratory phase and to calculate the average of three measurements.

To interpret obtained PAWP values sufficiently, we need to consider the influence of other clinical factors. For example, in patients with LHD, PAWP is often $<15 \mathrm{mmHg}$ because of the use of diuretics, in which differential diagnosis from PAH is important. It has been reported that rapid intravenous infusion of $500 \mathrm{~mL}$ normal saline is useful to distinguish LHD-associated pulmonary hypertension from PAH..$^{57}$

\section{4.11.4 Cardiac Output}

The gold standard for cardiac output measurement is the direct Fick method using practical measurement of oxygen consumption $\left(\mathrm{V}_{2}\right)$ and calculation based on the Fick principle. However, this method is not commonly used, because the process of $\mathrm{VO}_{2}$ measurement is complicated. In clinical practice, the indirect Fick method using estimated $\dot{\mathrm{V}} \mathrm{O}_{2}$ or the thermodilution method has been used as simplified methods. ${ }^{58}$ In the ESC/ERS Guidelines 2015, the thermodilution method is recommended based on the reports that the cardiac output measured by the thermodilution method in various settings significantly correlated with the value measured by the direct Fick method. However, several previous studies indicated that the cardiac output measured by the thermodilution method is misestimated in patients with low cardiac output or severe tricuspid insufficiency, in whom careful interpretation with reference to clinical symp- 
toms is required. Also, the cardiac output measured by the thermodilution method is incorrect in patients with intracardiac shunt. Basically, cardiac output is equal to pulmonary blood flow $(\mathrm{Qp})$. However, in patients with intra-cardiac shunt, cardiac output is not equal to Qp. Therefore, Qp is usually used to evaluate hemodynamic parameters of the pulmonary circulation in the field of pediatrics.

\subsubsection{Pulmonary Vascular Resistance (PVR)}

Wood units calculated from pressure $(\mathrm{mmHg})$ divided by flow rate $(\mathrm{L} / \mathrm{min})$ or the meter unit dynes $\cdot$ second $\cdot \mathrm{cm}^{-5}$ (Wood unit $\times 80$ ) have been used as a unit of pulmonary vascular resistance (PVR). At the Nice Conference in 2013, use of the Wood unit was recommended. Mean PAP is used for the definition of pulmonary hypertension, whereas the definition of pre-capillary PH employs PVR $>3.0$ Wood units in addition to PAWP $\leq 15 \mathrm{mmHg}$. PVR can be sensitively affected by both blood flow and pressure, and may not accurately reflect pulmonary circulation. On the other hand, the diastolic pressure gradient (DPG; pulmonary artery end-diastolic pressure-PAWP) is less affected by blood flow and pressure and may be more suitable for evaluation of pulmonary circulation in patients with LHD-associated pulmonary hypertension. However, the usefulness of DPG to predict outcome is still controversial. ${ }^{\mathbf{5 9}, 60}$

\section{4.11.6 Oxygen Saturation}

According to the Fick principle, when arterial oxygen saturation $\left(\mathrm{SaO}_{2}\right)$ and $\dot{\mathrm{VO}}_{2}$ are constant, mixed venous oxygen saturation $\left(\mathrm{SvO}_{2}\right)$ correlates with cardiac output, and $\mathrm{SvO}_{2}$ decreases with a reduction in cardiac output. Therefore, $\mathrm{SvO}_{2}$ has been used as a surrogate marker to estimate cardiac output, and has been used for risk classification of IPAH/HPAH patients (see Table 8). $\mathrm{SvO}_{2}$ is basically determined by measurement of blood oxygen saturation in pulmonary artery. In patients with left-to-right intra-cardiac shunt, $\mathrm{SvO}_{2}$ is higher than the normal value. If $\mathrm{SvO}_{2}$ is higher than $75 \%$, blood oxygen saturation of superior vena cava, inferior vena cava, and pulmonary artery needs to be measured to clarify the cause of $\mathrm{SvO}_{2}$ elevation.

\section{4.11.7 Acute Pulmonary Vasoreactivity Test}

The acute pulmonary vasoreactivity test is recommended to determine the indications of treatment with high-dose calcium $(\mathrm{Ca})$ channel blockers in patients with IPAH, HPAH, or drug-induced PAH.61 The usefulness of the acute pulmonary vasoreactivity test in other types of pulmonary hypertension remains unclear. For the acute pulmonary vasoreactivity test, inhaled nitrogen monoxide (NO) (10-20 ppm, 5 minutes) is recommended. Also, epoprostenol serial intravenous infusion $(2-12 \mathrm{ng} / \mathrm{kg} / \mathrm{min}$, dose increasing by $2 \mathrm{ng} / \mathrm{kg} / \mathrm{min}$ at intervals of 10 minutes), adenosine intravenous infusion $(50-350 \mu \mathrm{g} / \mathrm{kg} / \mathrm{min}$, dose increasing by $50 \mu \mathrm{g} / \mathrm{kg} / \mathrm{min}$ at intervals of 2 minutes), and iloprost inhalation (5-20 $\mu \mathrm{g}, 15$ minutes) are recommended as alternatives in the ESC/ERS Guidelines. ${ }^{1}$ In the acute pulmonary vasoreactivity test, a positive response is defined as a reduction of the mean PAP $\geq 10 \mathrm{mmHg}$ and decreasing absolute value of the mean PAP $\leq 40 \mathrm{mmHg}$ with an increased or unchanged cardiac output. ${ }^{61}$ The patients who meet these criteria are rare. According to the ESC/ERS Guidelines, the positive cases can be treated with Ca channel blockers, however, those can show good response to other vasodilators. Thus, positive cases can be treated with Ca channel blockers, which are lower in price than other pulmonary vasodilators. Furthermore, this test is potentially useful to predict the response to other pulmonary vasodilators prior to initiation of treatment.

\subsection{Pulmonary Angiography}

Pulmonary angiography (PAG) is performed for a definite diagnosis of CTEPH and determination of indications for pulmonary endarterectomy (PEA) or balloon pulmonary angioplasty (BPA). It is also useful in distinction from pulmonary artery disease originating from Takayasu's disease (a disease analogous to CTEPH) or from peripheral pulmonary artery stenosis. However, PAG is not generally indicated in PAH.

\subsubsection{Venous Puncture}

Puncture sites include the internal jugular vein, femoral vein, brachial vein, and subclavicular vein. Of these veins, the right internal jugular vein or the right femoral vein is often selected. Venous puncture under ultrasound guidance can reduce complications such as hematoma, erroneous arterial puncture, and pneumothorax.

\section{4.12.2 Catheter Manipulation}

The pigtail catheter or the balloon-tip catheter is advanced into the proximal segment of the right and left pulmonary arteries to separately conduct angiography. The French size of the catheter is important in achieving catheter stability and high-quality imaging. The guide wire is inserted into the pulmonary artery main trunk and along this wire the pigtail catheter is advanced into the pulmonary artery main trunk. As a precaution in this step, if the guide wire is inserted too deeply into the peripheral pulmonary artery, injury may be occur, resulting in reduced oxygenation due to bloody sputum/hemoptysis, occasionally requiring non-invasive positive airway pressure ventilation (NPPV) or mechanical ventilation. There are also cases in which the guide wire erroneously enters the coronary sinus instead of the right ventricle via the right atrium.

\subsubsection{Imaging}

After the pigtail is advanced into the proximal segment of bilateral pulmonary arteries and its position is adjusted, digital subtraction angiography (DSA) or digital angiography (DA) is performed during deep inhalation. If respiration can be adjusted appropriately, DSA provides high resolution imaging with a small volume of contrast material, and the consistency rate of diagnosis among different examiners has also been reported to be high. In comparison to DSA, DA requires approximately $20-30 \%$ more contrast material and more experience of imaging but is superior in terms of spatial and temporal resolution, and judging the texture of pulmonary artery lesions and the delay in contrast enhancement. Because DA can be a reference for BPS, an increasing number of facilities have adopted DA after introduction of BPA as a new treatment alternative. Imaging is often performed in two directions, i.e., frontal and lateral (90 degrees). As needed, oblique imaging is added to these directions to isolate the segmental branches of the pulmonary artery. The volume of contrast material used for DA is $12-15 \mathrm{~mL}$ per second and about $30-35 \mathrm{~mL}$ in total.

Selective PAG can reduce the load on the heart caused by the contrast material and is therefore considered for 


\begin{tabular}{|l|l|}
\hline \multicolumn{2}{|c|}{ Table 7. Functional Classification of Pulmonary Hypertension } \\
\hline NYHA Functional Classification \\
\hline Class I & No symptoms in ordinary physical activity \\
\hline Class II & $\begin{array}{l}\text { Symptoms and slight limitation during ordinary } \\
\text { physical activity }\end{array}$ \\
\hline Class III & $\begin{array}{l}\text { Symptoms and marked limitation during less-than- } \\
\text { ordinary physical activity }\end{array}$ \\
\hline Class IV & Symptoms during any physical activity and even at rest \\
\hline WHO Functional Classification of Pulmonary Hypertension \\
\hline Class I & $\begin{array}{l}\text { Patients with pulmonary hypertension in whom there } \\
\text { is no limitation of usual physical activity; ordinary } \\
\text { physical activity does not cause increased dyspnea, } \\
\text { fatigue, chest pain, or presyncope }\end{array}$ \\
\hline Class II & $\begin{array}{l}\text { Patients with pulmonary hypertension who have mild } \\
\text { limitation of physical activity. There is no discomfort } \\
\text { at rest, but normal physical activity causes increased } \\
\text { dyspnea, fatigue, chest pain, or presyncope }\end{array}$ \\
\hline Class III & $\begin{array}{l}\text { Patients with pulmonary hypertension who have a } \\
\text { marked limitation of physical activity. There is no } \\
\text { discomfort at rest, but less than ordinary activity } \\
\text { causes increased dyspnea, fatigue, chest pain, or } \\
\text { presyncope }\end{array}$ \\
\hline $\begin{array}{l}\text { Patients with pulmonary hypertension who are unable } \\
\text { to perform any physical activity at rest and who may } \\
\text { have signs of right ventricular failure. Dyspnea and/ } \\
\text { or fatigue may be present at rest and symptoms are } \\
\text { increased by almost any physical activity }\end{array}$ \\
Class IV
\end{tabular}

(Source: Barst RJ, et al. 2004,80 Rich S. Primary pulmonary hypertension: executive summary. Evian, France: World Health Organization, 1998)

pulmonary hypertension associated with right-sided heart failure (NYHA/WHO Functional Class IV). A pigtail catheter or a balloon-tip catheter is advanced into each lobe artery or the segmental branch to perform selective angiography. Angiography of one branch requires about $5 \mathrm{~mL}$ contrast material. For selective PAG, anatomical understanding is essential. If possible, prior determination of the location should be performed by CT scan to facilitate smooth implementation of this procedure.

\subsubsection{Complications}

In the PIOPED Study involving 1,111 patients, the mortality rate from PAG was $0.5 \%$, and many of the patients who died were reported to have had reduced function in the right heart system. ${ }^{62}$ At present, nonionic contrast material is extensively used, improving the safety of this procedure. Other possible complications include allergy, renal dysfunction, puncture site hematoma, heart failure, and arrhythmia. PAG can cause transient right bundle branch block, requiring caution in patients with left bundle branch block.

\subsection{Lung Biopsy}

Pathological findings are important in a definite diagnosis of PVOD/PCH. However, from the risk-benefit point of view, lung biopsy for the purpose of a definite diagnosis or differential diagnosis of pulmonary hypertension is not usually recommended. ${ }^{63,64}$ It has been reported that the pulmonary vessel remodeling at the time of lung biopsy during surgery for CTEPH cases correlates with the postoperative prognosis or with the long-term postoperative pulmonary hemodynamics. ${ }^{\mathbf{6 5}, 66}$

For patients with congenital heart disease exhibiting a shunt in the borderline range of indications for surgery, there is a view that the pathological findings from lung biopsy are valuable in determining the indications. ${ }^{1,20}$ In Japan, Yamaki et al. in $1976^{67}$ devised the "index of pulmonary vascular disease (IPVD)" as an indicator of pathological findings of pulmonary arterioles correlating with the severity of pulmonary hypertension on the basis of the pathological findings of pulmonary arterioles in autopsied cases of complete transposition of the great arteries and ventricular septal defect. This index differs from the severity grading of pulmonary arterioles associated with congenital heart disease proposed by Heath and Edwards in 1958. ${ }^{68}$ Yamaki et al. found that, there was no difference in clinical severity among Grades 4 to 6 of the Heath-Edwards Classification and conflicting evaluation was performed on the most advanced lesion in patients with a mixture of lesions at different grades, and then demonstrated the usefulness of using IPVD, an average score calculated on the basis of pathological findings of pulmonary arterioles, for evaluation of the indications for surgery in borderline cases of severe pulmonary hypertension. ${ }^{69}$ They also reported results using IPVD of pulmonary vascular changes in leftto-right shunt diseases such as ASD, PDA and complete atrio-ventricular septal defect, and several CHDs associated with Down's syndrome. In 1987, they showed the usefulness of evaluation by IPVD to decide the indications for surgery in ASD with severe pulmonary hypertension. ${ }^{69}$ In Japan, even at present, the pediatric cardiologists and pediatric cardiovascular surgeons often perform lung biopsy for borderline cases and make a final judgment from pathological findings of pulmonary arterioles when determining the indications of radical surgery for children with congenital heart disease associated with shunt and severe pulmonary hypertension. ${ }^{70}$

\subsection{Exercise Testing}

Many clinical studies demonstrated that the improvement of exercise capacity is associated with the improvement of prognosis. Therefore, exercise capacity has been examined as a noninvasive surrogate indicator of the conventional hemodynamic parameters.

\subsubsection{6-Minute Walk Test}

It has been reported that the 6-minute walk distance (6MWD) is closely related to hemodynamic parameters at the baseline, ${ }^{71}$ and has been used as a primary endpoint in many clinical studies. This is a very simple indicator and the method for measurement is standardized; it remains to be one of the major variables even at present. However, meta-analysis of the data from 22 clinical studies demonstrated that changes in 6MWD following treatment have not predicted the occurrence of clinical events. ${ }^{72}$ Evaluation of the therapeutic effects using the changes in 6MWD is insufficient in patients with preserved exercise capacity at baseline because of low sensitivity of 6MWD. It is also known that 6MWD is affected by learning effects, patient's characteristics, willingness, and comorbidities, thus, careful attention to interpret the values of 6MWD is required. Currently, the cut-off value of $6 \mathrm{MWD}>440 \mathrm{~m}$, which is proposed based on the results of REVEAL registry, has been used as a treatment goal, because it included the largest patients number among this kind of registry studies. ${ }^{73}$ However, this value may be inappropriate for young patients. Furthermore, since previous studies indicated several different cut-off values for prediction of the out- 
Symptoms, disease history, physical findings suggesting $\mathrm{PH}$

Possibility of PH suggested by echocardiography

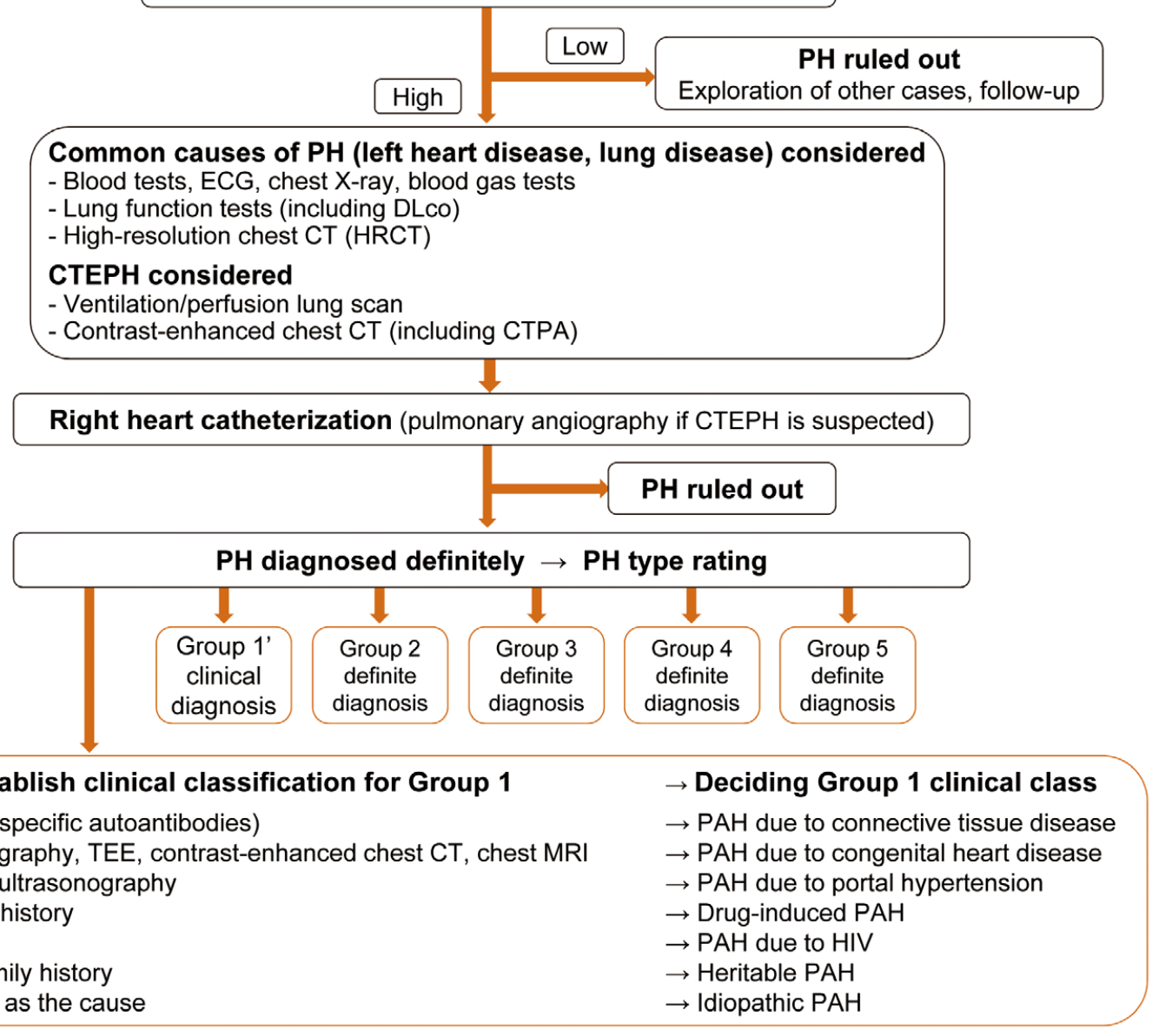

Figure 1. Diagnostic algorithm for pulmonary hypertension.

come, it might be difficult to decide which values are appropriate as a prognostic indicator. ${ }^{74-76}$

\section{4.14.2 Cardiopulmonary Exercise Testing}

It has been reported that patients with maximum oxygen consumption $\leq 10.4 \mathrm{~mL} / \mathrm{min} / \mathrm{kg}$ and maximum systolic blood pressure $\leq 120 \mathrm{mmHg}$ in the cardiopulmonary exercise testing (CPX) showed poor prognosis. ${ }^{77}$ It has also been reported that the appearance of right-to-left shunt during exercise affects the prognosis. ${ }^{78}$ Various reports have demonstrated that CPX is useful to predict the outcome of patients with pulmonary hypertension. At the Nice Conference (2013), maximum oxygen consumption $>15 \mathrm{~mL} / \mathrm{min} /$ $\mathrm{kg}$ and $\dot{\mathrm{VE}} / \mathrm{V}^{\mathrm{V} C O} \mathrm{O}_{2}$ at the anaerobic threshold $<45$ were proposed as the goals of treatment. ${ }^{79}$

\subsection{Functional Classification of Pulmonary Hypertension}

Both the NYHA Heart Functional Classification and the WHO Functional Classification of Pulmonary Hyperten- sion $^{80}$ shown in Table 7 have been used for severity grading based on clinical symptoms of pulmonary hypertension. Individual severity levels are approximately consistent between these two classifications. In these guidelines, the NYHA Heart Functional Classification was combined with the WHO Functional Classification of Pulmonary Hypertension to yield "NYHA/WHO Functional Class" which was then used as a functional classification of pulmonary hypertension, as in the previous guidelines.

\subsection{Diagnostic Algorithm}

The diagnosis of pulmonary hypertension begins when pulmonary hypertension is suspected on the basis of disease history and physical findings (Figure 1). Echocardiography, which is noninvasive and simple, is used to examine the possibility of pulmonary hypertension (see 4.4 Echocardiography for Detailed Findings).

If the probability of pulmonary hypertension is suggested to be high by echocardiography, examinations, such as blood tests, electrocardiography, chest X-ray, blood gas 
tests, lung function tests (including DLco), high-resolution computed tomography (HRCT), ventilation/perfusion lung scan, and contrast-enhanced chest CT (including pulmonary angiography (PAG) by CT: CTPA), are carried out (see another section for details of test findings).

Subsequently, hemodynamics is evaluated by right heart catheterization to make a definite diagnosis of pulmonary hypertension. If mean PAP at rest is $\geq 25 \mathrm{mmHg}$, a diagnosis of pulmonary hypertension is made. For the diagnosis of $\mathrm{PAH}$, the requirement PAWP $\leq 15 \mathrm{mmHg}$ needs to be additionally satisfied. If Group 4 pulmonary hypertension (CTEPH) is suspected on the basis of the results of ventilation/perfusion lung scan, pulmonary angiography is performed at the time of right heart catheterization and if it yields characteristic radiographic findings on PAG, a definite diagnosis is possible. In addition, the therapeutic strategy, such as indications for surgical treatment, is determined from the location and morphology of the lesions.

In addition to the results of right heart catheterization, the results of echocardiography (in case of Group 2 pulmonary hypertension) or lung function test including DLco (in case of Group 3 pulmonary hypertension) are additionally used to make a definite diagnosis.

For Group 1 pulmonary hypertension (PAH), differential diagnosis for distinction from the underlying disease is conducted as well. The diagnosis of CTD-PAH is possible on the basis of disease history, physical findings, and blood tests (various specific autoantibodies). The diagnosis of CHD-PAH is possible on the basis of echocardiography, transesophageal echocardiography, contrast-enhanced chest CT, and chest MRI. The diagnosis of PAH and other disease associated with portal hypertension is possible on the basis of abdominal ultrasonography. In addition, medication history and HIV testing are needed for differential diagnosis of PAH. In cases in which the above-mentioned diseases have been ruled out, detailed family history is taken and genetic tests (BMPR2) are carried out for diagnosis of HPAH, and a diagnosis of IPAH is made if no underlying disease as a cause is found.

The possibility of Group 1' PVOD/PCH (a subtype of $\mathrm{PAH}$ ) also needs to be considered. PVOD/PCH exhibits hemodynamics similar to PAH, but DLco is low and characteristic features are also visualized by HRCT. Although a definite diagnosis is based on histopathological findings, the clinical diagnosis is important since $\mathrm{PVOD} / \mathrm{PCH}$ differs from $\mathrm{PAH}$ in terms of therapeutic strategy.

Pulmonary hypertension complicating hematological disease (e.g., myeloproliferative disease), systemic disease (e.g., sarcoidosis and vasculitis), or metabolic disease is classified as Group 5. Further differential diagnosis of each group is discussed in the itemized statements.

\section{Descriptions}

\section{Pulmonary Arterial Hypertension}

\section{I1.1 Idiopathic Pulmonary Arterial Hypertension (IPAH)/ Heritable Pulmonary Arterial Hypertension (HPAH) \\ Summary}

Idiopathic pulmonary arterial hypertension (IPAH) and heritable pulmonary arterial hypertension (HPAH) are quite rare diseases without an apparent underlying disease and are characterized by severe pulmonary hypertension. They are seen more frequently in females, with the male/ female ratio being 1:1.7. The age upon their onset is young and they frequently develop in females at childbearing age. ${ }^{81}$ The incidence of these diseases is 1 to 2 out of 1 million population. In patients receiving no therapeutic intervention, the mean survival period after diagnosis was 2.8 years, indicating very poor prognosis. ${ }^{82}$

There was no established means of treatment in the past. In the 1990 s, however, prostaglandin analogues began to be administered clinically, and new drugs for treatment were developed one after another after 2005. At present, three drugs with different mechanisms are available, and the prognosis of patients with these diseases has been improved by treatment of one or a combination of these drugs. In cases in which drug resistance has developed, however, lung transplantation needs to be considered at an appropriate timing. Among all types of pulmonary arterial hypertension (PAH), HPAH is the only type showing known genetic mutations or familial onset, and is thus classified as a disease with genetic involvement. On the other hand, the etiology remains unknown for IPAH.,10,83 85

\subsubsection{Epidemiology and Etiology}

\section{a. Epidemiology}

In Japan, the disease previously called "primary pulmonary hypertension" (corresponding to IPAH/HPAH according to the current nomenclature) was listed as a disease covered by the specific disease treatment study program (so-called "intractable disease") in 1998, and an epidemiological survey using the clinical survey form for individual cases was started in that year. This survey is the only source of official and nationwide epidemiological information in Japan. "Primary pulmonary hypertension" listed as a disease covered by the specific disease treatment study program was renamed in October 2009 as PAH (a disease concept of global standard). At that time, registration as cases of intractable disease PAH was started to include not only patients with IPAH/HPAH but also patients with $\mathrm{PAH}$ associated with other PAH such as connective tissue disease, congenital heart disease. According to the statistics in 2015, the number of registered patients with PAH was 2,999 . If the disease responsible for PAH in these cases is analyzed over time, the number of patients with IPAH/ HPAH in Japan will be clarified.

Regarding the overseas epidemiological information, the first attempt of registration of cases of primary pulmonary hypertension in the United States reported the incidence of this disease to be 1 to 2 out of 1 million population. ${ }^{86}$ According to a study of national registration in France, 674 patients with $\mathrm{PAH}$ aged 18 and over were registered in 2002 , including 290 patients with IPAH/HPAH ( $43 \%$ of all PAH cases). This allowed a calculation that the prevalence of PAH was 15.0 out of 1 million population, including 5.9 cases of IPAH. ${ }^{11}$ According to the REVEAL registry study in the United States, 2,525 cases of PAH were registered during the 1.5-year period from 2006 to 2007, including 


\begin{tabular}{|c|c|c|c|}
\hline $\begin{array}{l}\text { Prognostic factor (estimated } \\
\text { mortality rate at } 1 \text { year) }\end{array}$ & $\begin{array}{l}\text { Low risk } \\
(<5 \%)\end{array}$ & $\begin{array}{l}\text { Medium risk } \\
(5-10 \%)\end{array}$ & $\begin{array}{l}\text { High risk } \\
(>10 \%)\end{array}$ \\
\hline $\begin{array}{l}\text { Clinical sign of right-sided } \\
\text { heart failure }\end{array}$ & Absent & Absent (or present) & Present \\
\hline Symptom exacerbation & Absent & Slow & Rapid \\
\hline Syncope & Absent & Incidental & Repeated \\
\hline NYHA/WHO functional class & I, II & III & IV \\
\hline 6-minute walk distance & $>440 m$ & $165-440 m$ & $<165 \mathrm{~m}$ \\
\hline $\begin{array}{l}\text { Cardiopulmonary exercise } \\
\text { load test }\end{array}$ & $\begin{array}{c}\text { peak } \dot{\mathrm{VO}}_{2}>15 \mathrm{~mL} / \mathrm{min} / \mathrm{kg} \\
(>65 \% \text { predicted) } \\
\dot{\mathrm{V} E} / \mathrm{VCO}_{2} \text { slope }<36\end{array}$ & $\begin{array}{c}\text { peak } \dot{\mathrm{VO}}_{2} 11-15 \mathrm{~mL} / \mathrm{min} / \mathrm{kg} \\
(35-65 \% \text { predicted) } \\
\dot{\mathrm{V} E} / \mathrm{VCO}_{2} \text { slope } 36-44.9\end{array}$ & $\begin{array}{c}\text { peak } \dot{\mathrm{V}} \mathrm{O}_{2}<11 \mathrm{~mL} / \mathrm{min} / \mathrm{kg} \\
(<35 \% \text { predicted) } \\
\dot{\mathrm{V} E} / \dot{V}^{\prime} \mathrm{CO}_{2} \text { slope } \geq 45\end{array}$ \\
\hline $\begin{array}{l}\text { BNP } \\
\text { NT-proBNP }\end{array}$ & $\begin{array}{c}\text { BNP <50ng/L } \\
\text { NT-proBNP <300ng/L }\end{array}$ & $\begin{array}{c}\text { BNP 50-300 ng/L } \\
\text { NT-proBNP } 300-1,400 \mathrm{ng} / \mathrm{L}\end{array}$ & $\begin{array}{c}\text { BNP }>300 \mathrm{ng} / \mathrm{L} \\
\text { NT-proBNP }>1,400 \mathrm{ng} / \mathrm{L}\end{array}$ \\
\hline $\begin{array}{l}\text { Imaging (echocardiography, } \\
\text { heart MRI) }\end{array}$ & $\begin{array}{l}\text { Right atrium area }<18 \mathrm{~cm}^{2} \\
\text { No endocardial fluid }\end{array}$ & $\begin{array}{l}\text { Right atrium area } 18-26 \mathrm{~cm}^{2} \\
\text { No or little endocardial fluid }\end{array}$ & $\begin{array}{c}\text { Right atrium area }>26 \mathrm{~cm}^{2} \\
\text { Endocardial fluid present }\end{array}$ \\
\hline $\begin{array}{l}\text { Hemodynamics } \\
\text { (catheterization) }\end{array}$ & $\begin{array}{c}\text { Mean right atrial pressure }<8 \mathrm{mmHg} \\
\text { Cardiac index } \geq 2.5 \mathrm{~L} / \mathrm{min} / \mathrm{m}^{2} \\
\mathrm{SvO}_{2}>65 \%\end{array}$ & $\begin{array}{c}\text { Mean atrial pressure } 8-14 \mathrm{mmHg} \\
\text { Cardiac index } 2.0-2.4 \mathrm{~L} / \mathrm{min} / \mathrm{m}^{2} \\
\mathrm{SvO}_{2} 60-65 \%\end{array}$ & $\begin{array}{c}\text { Mean atrial pressure }>14 \mathrm{mmHg} \\
\text { Cardiac index }<2.0 \mathrm{~L} / \mathrm{min} / \mathrm{m}^{2} \\
\mathrm{SvO}_{2}<60 \%\end{array}$ \\
\hline
\end{tabular}

(Source: Prepared based on Galiè N, et al. 2016¹)

1,166 cases of IPAH (46\% of all PAH cases). ${ }^{\mathbf{8 7}}$ Because the entire population in the USA at that time was about 300 million, the prevalence of PAH was calculated to be 8.4 out of 1 million population, including 3.9 cases of IPAH.

\section{b. Etiology}

Factors reported to date to be possibly associated with IPAH include inflammation, growth factor, Ca signaling, bone morphogenic protein (BMP) receptor/transforming growth factor $\beta$ (TGF- $\beta$ ) pathway abnormality, neuroendocrine abnormality, abnormal angiogenesis, abnormal vascular metabolism, abnormal mitochondrial function, abnormal extracellular matrix, and vasoactive material anomaly. ${ }^{88-90}$ A question is whether these abnormalities are the cause or outcome of IPAH. In cases of IPAH/HPAH, abnormality of the genes encoding the BMP receptor/ TGF- $\beta$ pathway has been seen reported, ${ }^{\mathbf{1 0 , 8 5 , 9 1}}$ and it has been shown that a decrease in BMP matrix can be a cause for IPAH/HPAH even in the absence of abnormality in these genes.92 Furthermore, treatment with drugs that increase or stimulate the BMP matrix has begun on a trial basis. ${ }^{93,94}$

\section{1.1.2 Diagnosis}

Diagnosis of IPAH/HPAH follows the procedure described in the Outline (see Figure 1). Briefly, the diagnosis of pulmonary hypertension consists of three factors (presence diagnosis, differential diagnosis, and severity rating). In many cases, IPAH/HPAH develops with initial symptoms of dyspnea on exertion, generalized malaise, chest pain, and syncope.

If the presence of pulmonary hypertension is suspected on the basis of ECG and/or chest X-ray, echocardiography/ Doppler ultrasonography is carried out for a definite diagnosis and semi-quantitative evaluation of pulmonary hypertension. For distinction among different types of pulmonary hypertension, the presence of left heart disease and lung disease (including hypoxemia) is first examined. If these diseases are ruled out, the probability for the presence of Group 1 (PAH) or Group 4 (chronic thromboembolic pulmonary hypertension: CTEPH) is judged to be high. For evaluation of the latter possibility, ventilation/ perfusion lung scan is useful, whereas accurate judgment is difficult with conventional contrast-enhanced CT. If the possibility of PAH is high, blood tests and diagnostic imaging are used to rule out $\mathrm{PAH}$ due to connective tissue disease (CTD-PAH), portopulmonary hypertension (PoPH), PAH due to congenital heart disease (CHD-PAH), and pulmonary veno-occlusive disease (PVOD)/pulmonary capillary hemangiomatosis $(\mathrm{PCH})$. Interpretation of the results of these tests is often difficult and it is necessary to obtain an accurate diagnosis at well-experienced facilities.

Pulmonary hemodynamics are measured by right heart catheterization for the purpose of a definite diagnosis and severity rating of $\mathrm{PAH}$. To determine therapeutic strategy, evaluation of exercise tolerance is also useful. For distinction between IPAH and HPAH, detailed disease history is first taken during clinical practice. Regarding genetic diagnosis, there are several facilities that conduct tests for this purpose in Japan and a definite diagnosis is also possible at these facilities. However, since the finding of carrier (without disease onset) status may trigger anxiety about disease onset in the patient, genetic diagnosis should be carried out after arranging a system for providing genetic counseling.

Regarding the PAH diagnostic criteria and the criteria for official registration of intractable diseases, reference should be made to "IV. Pulmonary hypertension under the measures against intractable diseases; (1) Pulmonary arterial hypertension (Listed Intractable Disease No. 86)" (see Document 1) of these guidelines.

\subsubsection{Prognosis/Severity Rating \\ a. Prognosis}

The natural course of IPAH/HPAH is quite poor. According to a report from the United States before 2000 when no effective drug for treatment was available, the mean survival period after onset of these diseases was 2.8 years in untreated adults, with the frequent cause of death being sudden death, right-sided heart failure and hemoptysis. ${ }^{\mathbf{8 2}}$

According to the nationwide data in Japan concerning the natural course of IPAH/HPAH, the 5-year survival rate of the 201 patients registered between 1980 and 1990 was $42.5 \% .^{95}$ According to the results of recent analyses of 
large-scale case registry data in Western countries, the prognosis has been improving, with the one-year, threeyear, and five-year survival rates of patients with IPAH/ HPAH/drug-induced PAH being $89 \%, 77 \%$, and $69 \%$, respectively, in a report from France ${ }^{96}$ and the one-, three-, five-, and seven-year survival rates being $91 \%, 74 \%, 65 \%$, and $59 \%$, respectively, in the RVEAL registry study in the USA. ${ }^{97}$ In a recent multicenter retrospective study in Japan, the one-, three-, five-, and ten-year survival rates of patients with IPAH/HPAH were $97.9 \%, 92.1 \%, 85.8 \%$, and $69.5 \%$, respectively, higher than the rates reported from other countries. ${ }^{98}$ This difference may be attributable to the recent development of specific pulmonary vasodilators, rapid and sufficient dose increase of epoprostenol, and other reasons.

\section{b. Severity/Prognosis Rating}

In the past, the rating of severity/prognosis of IPAH/ HPAH laid emphasis on pulmonary hemodynamic parameters, and an equation for prediction of the outcome based on these parameters was proposed. ${ }^{\mathbf{8 2}}$ In recent years, however, a predominant approach to severity/prognosis rating and therapeutic strategy determination involves severity evaluation and therapeutic efficacy judgment based on general assessment of the degree of disease progression, presence/absence of clinical symptoms (history of syncope, right-sided heart failure), New York Heart Association (NYHA)/World Health Organization (WHO) Functional Classification of Pulmonary Hypertension, exercise testing (6-minute walk test/cardiopulmonary exercise testing), ${ }^{71,99}$ physiological indicators, such as the presence/absence of pericardial fluid and tricuspid annular plane systolic excursion (TAPSE) rated by echocardiography, ${ }^{100}$ and biomarkers such as brain natriuretic peptide (BNP) ${ }^{101}$ and blood uric acid level. ${ }^{34}$ The Guidelines for the Diagnosis and Treatment of Pulmonary Hypertension 2015 prepared by the European Society of Cardiology (ESC)/European Respiratory Society (ERS) refer to these parameters and information when determining the severity, prognosis, and therapeutic strategy (Table 8), ${ }^{1}$ classifying the prognosis into low risk, medium risk, and high risk (in the descending order of the number of parameters known as favorable prognostic indicators). ${ }^{2}$ Furthermore, in the REVEAL registry study in the USA, a new risk score calculation method for prediction of the outcome of PAH was published. ${ }^{102}$ Concerning indicators of pulmonary circulation, these approaches of evaluation lay greater emphasis on the right atrial pressure, cardiac index, and pulmonary vascular resistance than on the pulmonary arterial pressure itself, and mixed venous oxygen saturation $\left(\mathrm{SvO}_{2}\right)$ may be useful in the evaluation of severity. In Japan, however, the relationship between mean pulmonary arterial pressure and prognosis has been given importance, and the prognosis has been improved markedly by adopting this as an indicator of treatment. ${ }^{103}$ In practice, it has been shown that lower mean pulmonary arterial pressure is associated with more favorable prognosis.

\subsubsection{Genetics in PAH}

The plexiform lesion seen in the pathological specimens of the lungs from PAH patients shows signs of proliferation of apoptosis-resistant vascular wall cells. Factors reported to underlie such abnormal proliferation of cells include abnormality of the cell proliferation regulation pathway, such as the gene $B M P R 2$ encoding the BMP receptor type
II (a member of the TGF- $\beta$ receptor superfamily), the gene $A C V R L 1$ encoding the activin receptor-like kinase (ALK)1 , and the gene encoding the intracellular signal transmitter SMAD, as well as abnormal gene polymorphism of the promoter region of serotonin transport protein (5-HTT) (a modifying gene) and of the angiotensin-converting enzyme (ACE) or endothelial nitrogen monoxide synthetase (eNOS). Furthermore, in cases of PAH accompanied by hereditary hemorrhagic telangiectasia (HHT), mutation of gene $A C V R L 1$ or $E N G$ has been reported, and mutation of these genes has been found also in PAH patients without HHT symptoms or family history. These findings suggest that a vasoproliferative disease in which mutation of the gene encoding BMPR 2 or ALK1 causes monoclonal proliferation of cells, resulting in resistance to apoptosis. ${ }^{104}$

In PAH patients with a family history, mutation of either $B M P R 2$ gene or $A C V R L 1$ gene is often detected. In patients with no mutation of these genes, mutation of SMAD8 gene has been detected. ${ }^{105}$ In recent years, reports have been published concerning the association of caveolin-1 ( $C A V 1)$ gene mutation and onset of $\mathrm{PAH}^{83}$ and onset of PAH triggered by Notch3 signals. ${ }^{106}$ There is a report also concerning the mechanism for cell proliferation following increased $\mathrm{Ca}^{2+}$ levels in vascular smooth muscle cells due to mutation of the transient receptor potential canonical 6 (TRPC6) channel. ${ }^{107}$ The theory attributing this disease to human herpes virus 8 (HHV-8) has been negatively considered. ${ }^{108}$

Regarding BMPR2 gene mutation, numerous studies have been published since the report of the association with PAH onset in $2000,{ }^{9}$ including a report that $B M P R 2$ gene mutation is present in about $70-80 \%$ of all patients with familial PAH and about $20-30 \%$ of patients with solitary PAH. ${ }^{109}$ In Japan, the prevalence of $B M P R 2$ gene mutation in Japanese patients is comparable to that found in overseas reports. ${ }^{110}$ It has also been reported that $B M P R 2$ gene mutation is not limited to the one inherited from parents but can also develop as a new mutation despite absence in the parents, leading to onset of PAH. ${ }^{111}$

According to the meta-analysis in Asia, Europe, and North America reported in 2016, BMPR2 gene mutation was seen in about $30 \%$ of PAH patients, and the group of patients possessing this mutation was lower in terms of the age upon diagnosis of the disease and severer in terms of hemodynamics, accompanied by the finding that the incidence of events (lung transplantation or death after diagnosis) was significantly higher in the $B M P R 2$ gene mutation group than in the mutation-free group of patients if the age upon diagnosis was less than 50.91 However, according to a report in Japan, the analysis limited to cases requiring continuous treatment with prostacyclin $\left(\mathrm{PGI}_{2}\right)$ after 2005 (the year when multiple-drug combination therapy became predominant for PAH treatment) revealed significantly better prognosis after the start of treatment in the group with $B M P R 2$ gene mutation. ${ }^{112}$ This result appears contradictory to the finding from the abovementioned meta-analysis in Asia, Europe, and North America (higher incidence of events in the group with $B M P R 2$ gene mutation), but it may reflect the influence of various factors, including ethnic difference between Japan and other countries and difference in the timing of start and dose level of continuous $\mathrm{PGI}_{2}$ preparations. Thus, it is necessary to manage of this disease tailored to individual patients based on clinical differences related to the presence/ absence of gene mutation by accumulation of further 


\begin{tabular}{|l|c|c|}
\hline \multicolumn{2}{|c|}{ Table 9. Recommendation and Evidence Level Concerning } \\
General Measures for IPAH/HPAH
\end{tabular}

evidence.

Care needs to be taken of the fact that a genetic test is not indispensable and should not be required. This test should not be carried out in a careless manner because its results can affect the life course of the patients and their families. Performing this test should be considered after preparing for its implementation, involving a specialist team consisting of a physician specializing in genetics and a genetic councilor.

\subsubsection{Therapy}

\section{a. General Measures}

General measures for IPAH/HPAH patients is summarized in Table 9. For patients with IPAH/HPAH, appropriate advice about daily living is needed, and support for their family members and patient support groups are also important.

In the ESC/ERS Guidelines for the Diagnosis and Treatment of Pulmonary Hypertension 2015, supervised exercise training is considered to treat physical deconditioning of PAH patients. ${ }^{1}$ This recommendation was adopted on the basis of the randomized controlled trials (RCTs), which demonstrated significant improvement or alleviation of exercise capacity, physical activity, and fatigue in the group having received rehabilitation primarily consisting of exercise training. ${ }^{113-116}$ Other than these RCTs, there are a few reports on the effectiveness of exercise training, although most of them are based on small-scale evaluation. ${ }^{117-120}$ Adverse events arising from exercise training reported to date include syncope, presyncope, and supraventricular tachycardia. ${ }^{120}$ In an animal study using a rat model of severe pulmonary hypertension, exercise resulted in inflammatory cell infiltration of the right ventricle and remodeling of the pulmonary artery, leading to a lower survival rate. ${ }^{\mathbf{1 2 1}}$ Further studies are needed about the longterm influence of exercise training on pulmonary circulation and prognosis (survival). Furthermore, none out of the appropriate style, frequency, intensity, and duration of the exercise training for patients has been established. In patients who have been hemodynamically stable with drug therapy, it is reasonable to consider exercise training.

\begin{tabular}{|c|c|c|}
\hline \multicolumn{3}{|c|}{$\begin{array}{l}\text { Table 10. Recommendation and Evidence Level Concerning } \\
\text { Supportive Therapy for IPAH/HPAH }\end{array}$} \\
\hline Recommendation & $\begin{array}{c}\text { Recommendation } \\
\text { Class }\end{array}$ & $\begin{array}{l}\text { Evidence } \\
\text { Level }\end{array}$ \\
\hline $\begin{array}{l}\text { Diuretics for treatment of right- } \\
\text { sided heart failure and fluid } \\
\text { retention }\end{array}$ & 1 & $\mathrm{C}$ \\
\hline $\begin{array}{l}\text { Long-term oxygen supply in } \\
\text { cases of arterial oxygen pressure } \\
\text { less than } 60 \mathrm{mmHg}\end{array}$ & 1 & $\mathrm{C}$ \\
\hline $\begin{array}{l}\text { Oral anticoagulant therapy } \\
\text { (However, warfarin is not } \\
\text { recommended during use of } \\
\text { epoprostenol) }\end{array}$ & Ilb & $\mathrm{C}$ \\
\hline $\begin{array}{l}\text { Correction of anemia and/or iron } \\
\text { deficiency }\end{array}$ & $\mathrm{llb}$ & B \\
\hline $\begin{array}{l}\text { Administration of angiotensin- } \\
\text { converting enzyme inhibitors, } \\
\text { angiotensin II receptor antago- } \\
\text { nists and } \beta \text {-blockers } \\
\text { (However, careful determination } \\
\text { of indications is needed in } \\
\text { patients with accompanying } \\
\text { disease such as hypertension, } \\
\text { coronary artery disease, and } \\
\text { left-sided heart failure.) }\end{array}$ & III & $\mathrm{C}$ \\
\hline
\end{tabular}

However, exercise training should be carried out carefully while monitoring vital signs at facilities well experienced with treatment and rehabilitation of patients with pulmonary hypertension.

The mortality rate during pregnancy or delivery is still high (although showing a tendency for reduction) in wellcontrolled IPAH/HPAH patients, ${ }^{122}$ indicating that pregnancy is still a contraindication. Elective surgery for IPAH/ HPAH involves a high risk (mortality rate $3.5 \%$ ) and, when such surgery is performed, epidural anesthesia rather than general anesthesia is recommended. Furthermore, the risk factors for death from this surgery have been shown as 6-minute walk distance (6MWD) less than $400 \mathrm{~m}$, and emergency surgery. ${ }^{123}$ Because pneumonia affects the prognosis of $\mathrm{PAH}$ patients, ${ }^{\mathbf{8 1}}$ prior vaccination (influenza vaccine and pneumococcal vaccine) is recommended. Consideration is also needed about psychosocial supports, genetic counselling, and advice as to medication and travel for IPAH/HPAH patients.

There is no study concerning the need for supplemental oxygen during prolonged flight in IPAH/HPAH patients. However, the physical influence of hypoxemia is evident, and in-flight oxygen supply should be considered for IPAH/HPAH patients of NYHA/WHO Functional Class III/IV with arterial oxygen pressure less than $60 \mathrm{mmHg}{ }^{124}$

\section{b. Supportive Therapy}

Supportive therapy is summarized in Table $\mathbf{1 0}$ and is outlined below.

\section{i. Anticoagulant Therapy}

In three retrospective studies of IPAH/HPAH, as well as PAH patients receiving appetite-suppressants, the prognosis was better in the patients receiving warfarin than in the patients without warfarin treatment. ${ }^{\mathbf{1 2 5}-\mathbf{1 2 7}}$ For this reason, anticoagulant therapy with warfarin may be considered for IPAH/HPAH patients. In Japan, the warfarin dosage is often set at a level achieving the prothrombin time-international normalized ratio (PT-INR) in 1.5 to 2.5 range. 


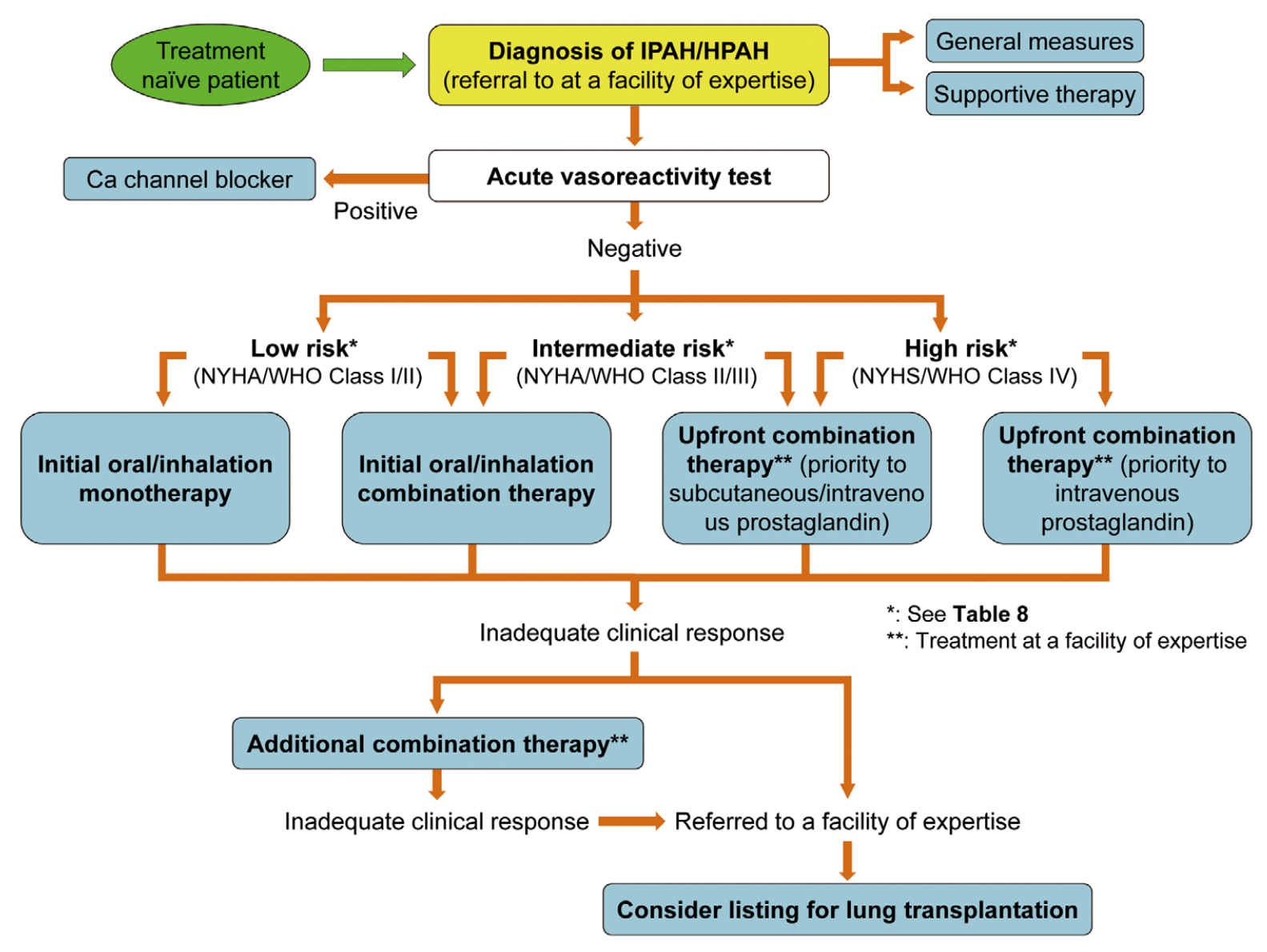

Figure 2. Therapeutic strategy for IPAH/HPAH. When a method of treatment is selected, the mean pulmonary arterial pressure (the most important prognostic determinant) should always be taken into consideration. (Source: Prepared based on Galiè N, et al. 2016')

However, all of the results were obtained in an era when epoprostenol was not available. When continuous intravenous infusion of epoprostenol is performed, thrombosis of a catheter origin may arise, and the risk for hemorrhagic complication due to the marked suppression of platelet aggregation also needs to be considered. A study of Japanese patients by Ogawa et al. demonstrated poorer prognosis following a combination epoprostenol and warfarin therapy. ${ }^{\mathbf{9 8}}$ For this reason, in Japan the use of warfarin for IPAH/HPAH patients receiving epoprostenol is not recommended. Usefulness of direct oral anticoagulants (DOAC) in IPAH/HPAH patients remains unknown.

\section{ii. Diuretics}

Right-sided heart failure causes fluid retention, and diuretics are used for its control. Loop diuretics, thiazide diuretics and aldosterone antagonists are used alone or in combination. When these drugs are used, adequate care needs to be taken of electrolyte anomalies and hypotension possibly arising from intravascular dehydration as well as lowering cardiac output. In recent years, the usefulness of tolvaptan in the management of IPAH/HPAH was reported in Japan. ${ }^{128}$

\section{iii. Long-Term Oxygen Therapy (Ambulatory Oxygen Therapy)}

In patients with PAH, oxygen supply has an acute effect in reducing mean pulmonary arterial pressure and pulmonary vascular resistance. ${ }^{\mathbf{1 2 9}, 130} \mathrm{With}$ the expectation of prognosisimproving effects similar to those demonstrated in patients with chronic obstructive pulmonary disease, oxygen therapy is usually performed with a goal set at maintaining arterial oxygen pressure at $60 \mathrm{mmHg}$ or higher. However, in the only randomized comparative study performed to date in patients with CHD-PAH, the mortality rate was not reduced in the group that received 2-year nocturnal oxygen therapy. ${ }^{131}$

\section{iv. Digitalis Preparations}

Digitalis has been shown in an animal study to exert weak pulmonary vasoconstrictive activity. ${ }^{132}$ In evaluation of the acute effects in PAH patients, cardiac output increased but pulmonary vascular resistance remained unchanged. ${ }^{\mathbf{1 3 3}}$ However, chronic effects of digitalis preparations remain obscure, and digitalis is thus positioned as a drug to be considered when heart rate control in the presence of atrial fibrillation is needed. 


\begin{tabular}{|c|c|c|c|c|c|}
\hline $\begin{array}{l}\text { Recommendation } \\
\text { Class }\end{array}$ & $\begin{array}{l}\text { Evidence } \\
\text { Level }\end{array}$ & $\begin{array}{c}\text { NYHA/WHO } \\
\text { Class I }\end{array}$ & $\begin{array}{c}\text { NYHA/WHO } \\
\text { Class II }\end{array}$ & $\begin{array}{l}\text { NYHA/WHO } \\
\text { Class III }\end{array}$ & $\begin{array}{l}\text { NYHA/WHO } \\
\text { Class IV }\end{array}$ \\
\hline 1 & A or $B$ & - & $\begin{array}{l}\text { ERA po } \\
\text { PDE5 inhibitor po } \\
\text { sGC stimulator po } \\
\text { Selexipag po }\end{array}$ & $\begin{array}{c}\text { ERA po } \\
\text { PDE5 inhibitor po } \\
\text { sGC stimulator po } \\
\text { Epoprostenol iv } \\
\text { Treprostinil sc } \\
\text { Iloprost inhalation } \\
\text { Selexipag po }\end{array}$ & Epoprostenol iv \\
\hline Ila & C & - & - & $\begin{array}{l}\text { Upfront combination therapy } \\
\text { Treprostinil iv }\end{array}$ & $\begin{array}{l}\text { Upfront combination } \\
\text { therapy }\end{array}$ \\
\hline \multirow[b]{2}{*}{ Ilb } & B & - & - & Beraprost po & - \\
\hline & C & $\begin{array}{l}\text { Beraprost po } \\
\text { ERA (Ambrisentan) po } \\
\text { sGC stimulator po } \\
\text { Selexipag po }\end{array}$ & $\begin{array}{l}\text { Beraprost po } \\
\text { Iloprost inhalation }\end{array}$ & - & $\begin{array}{c}\text { Beraprost po } \\
\text { ERA (excluding Ambrisentan) po } \\
\text { PDE5 inhibitor po } \\
\text { lloprost inhalation } \\
\text { Treprostinil sc/iv }\end{array}$ \\
\hline
\end{tabular}

ERA: Macitentan, ambrisentan, bosentan. PDE5 inhibitors: Tadalafil, sildenafil. sGC stimulators: Riociguat. po: oral, iv: intravenous, sc: subcutaneous.

v. Other Cardiotonic Agents and Cardiovascular Agonists Catecholamines are sometimes used in the treatment of severe right-sided heart failure and the introduction of drug therapy for treatment of pulmonary hypertension. Dobutamine is selected for cases in which systemic blood pressure is relatively preserved. In cases in which systemic blood pressure is not preserved, dopamine is used alone or in combination with dobutamine. Before weaning from catecholamines, oral drugs for treatment of heart failure, such as pimobendan, docarpamine, and denopamine, are sometimes useful. There are no reports on the usefulness or safety in IPAH/HPAH patients concerning ACE inhibitors, angiotensin II receptor antagonists (ARB), or $\beta$-blockers.

\section{vi. Anemia and Iron Status}

Iron deficiency is often seen in IPAH/HPAH patients, and may be associated with reduction of exercise tolerability and increased mortality regardless of the severity of anemia. ${ }^{134}$ For this reason, regular monitoring of the iron status should be considered in IPAH/HPAH patients and, if iron deficiency is detected, potential abnormalities should be clarified. Because oral iron absorption is sometimes reduced in IPAH/HPAH patients, the intravenous administration of iron should also be considered.

\section{c. Specific Drug Therapy}

The strategy for IPAH/HPAH treatment is outlined in Figure 2. First, a definite diagnosis of IPAH/HPAH and its severity rating are carried out. Accuracy is essential in the diagnosis of PAH and IPAH/HPAH, and referral to a specialist is necessary at this step because diagnosis may be inaccurate when made at a facility without specialists. Then, the aforementioned general management/supportive therapy are started. In parallel to this step, it is recommended to perform an acute pulmonary vasoreactivity test using nitrogen monoxide (NO) inhalation or intravenous epoprostenol injection at the time of right heart catheterization for IPAH/HPAH patients. Details of the acute vasoreactivity test are given in the Section "Diagnosis/ evaluation of Pulmonary Hypertension" of Chapter I.

Historically, the acute pulmonary vasoreactivity test has been conducted for the purpose of determining the indica- tions of Ca channel blockers in individual cases. ${ }^{125}$ According to the recent study by Sitbon et al., which analyzed the percentage of patients with IPAH, HPAH, or drug-induced PAH testing positive for this test, $12.6 \%$ of all patients with IPAH were positive. About half of the positive cases $(6.8 \%$ of all subjects) showed stabilization of clinical symptoms in response to $\mathrm{Ca}$ channel blockers treatment for one year or longer, suggesting a longer survival period. ${ }^{61}$ In Japan, the percentage of patients with IPAH or HPAH testing positive in this test has been reported to be quite low, but there are some positive cases. In positive cases, treatment with Ca channel blockers should be first considered. If the vasoreactivity test is positive, pulmonary vasodilators should be administered in combination with $\mathrm{Ca}$ channel blockers.

In cases testing negative for acute vasoreactivity, treatment with pulmonary vasodilators should be started immediately. At present, pulmonary vasodilators of three different families are available, i.e., prostacyclin and its derivatives (belonging to the prostacyclin pathway), endothelin receptor antagonists (ERA) belonging to the endothelin pathway, and phosphodiesterase type-5 inhibitors (PDE5) and guanylate cyclase stimulators belonging to the NO family.

In Western countries, many clinical studies were carried out before and after marketing of these drugs concerning their effects as pulmonary vasodilators. At the Dana Point World Symposium in 2008, preparations were made for Pulmonary Hypertension Treatment Guidelines summarizing the evidence related to treatment in those days. At the Nice Conference in 2013, the Guidelines were completed by the addition of data on many other drugs, and published as the ECS/ERS Pulmonary Hypertension Diagnosis and

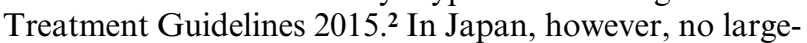
scale clinical study of pulmonary vasodilators has been carried out, and it is difficult to prepare PAH treatment guidelines unique to Japanese patients on the basis of evidence in Japanese. For this reason, although these guidelines were prepared based on these Western guidelines as a rule, the drugs used in Western countries but not yet marketed in Japan were excluded from the Guidelines, reflecting the evidence and experience in Japan.

In these guidelines, therapeutic strategy is shown pri- 
marily in accordance with the NYHA/WHO functional class (Figure 2, Table 11). NYHA/WHO functional class at the time of diagnosis is one of the prognostic factors. In the REVEAL registry study in the USA, the five-year survival rate was $86 \%$ for the $\mathrm{PAH}$ patients rated as NYHA/WHO functional class I at the time of diagnosis. The mean pulmonary arterial pressure for this group at the time of registration was as high as $50 \mathrm{mmHg} .{ }^{135} \mathrm{On}$ the other hand, in a study of 130 IPAH/HPAH patients at 3 Japanese facilities, the prognosis was significantly favorable in cases in which the mean pulmonary arterial pressure dropped to $46 \mathrm{mmHg}$ or less during follow-up. ${ }^{98}$ The NYHA/WHO functional class is based on the patient's symptoms and the examiner's subjective judgment and is therefore not always associated with severity level. Because the data collected to date in Japan indicate that the prognosis of PAH depends on the pulmonary arterial pressure, these guidelines recommend consideration of the use of pulmonary vasodilators in patients with high mean pulmonary arterial pressure even when the NYHA/WHO functional class is I.

As described above, there are three families of pulmonary vasodilators available. Treatment is started using one of these drugs. Most drugs are useful in cases rated as NYHA/ WHO functional class III and some drugs have been shown to be effective also in Class II cases (Table 11). In severe cases possessing many other indicators of high severity associated with poor prognosis (e.g., rapid progression of symptoms) among the cases rated as NYHA/WHO functional class III, the indications for epoprostenol should be considered. NYHA/WHO functional Class IV is indicated for epoprostenol.

In cases in which the acute vasoreactivity test is negative, the severity is graded in accordance with Figure 2, and the therapeutic strategy is determined on the basis of the thus rated severity level. For low-risk cases and medium-risk mild cases, oral-dose or inhalational drugs are selected from Table 11, taking into consideration possible complications. Depending on severity level and degree of improvement, drugs are used alone or in combination. For medium-risk severe cases (NYHA Class III, presenting with shortness of breath while walking on level ground or mPAP $40 \mathrm{mmHg}$ ), upfront combination therapy (mentioned later) is performed with priority given to intravenous or subcutaneous injection of prostaglandins. Because experience is needed for intravenous or subcutaneous use of prostaglandins and for upfront combination therapy, it is necessary to refer the patient to a facility with specialists. For high-risk cases, upfront combination therapy is performed with priority given to intravenous epoprostenol treatment and, if intravenous injection is difficult, subcutaneous injection is included in the upfront combination therapy. If such treatment does not lead to satisfactory improvement, lung transplantation is considered. In Figure 2, addition of some other drugs to the combined treatment is shown as an alternative for cases poorly responding to the upfront combination therapy. This alternative refers primarily to cases finally referred to a facility with specialists because of unsatisfactory improvement in response to the insufficient upfront combination therapy provided at a facility without specialists. In such cases, it is not uncommon that the treatment at the facility with specialists dealing with the referred patient involves difficulties. The therapeutic strategy mentioned above has been actually adopted at Japanese facilities specializing in pulmonary hypertension. It is still a strategy unique to
Japan, without being supported by strong evidence. However, the survival rate analyzed as an outcome measure was superior to that in Western countries where different strategies have been adopted. ${ }^{\mathbf{1 3 6}}$ For this reason, this strategy was adopted in these guidelines.

After publication of the previous version "Pulmonary Hypertension Treatment Guidelines 2012 Revised Version," the following pulmonary vasodilators became available in Japan: treprostinil, iloprost, and selexipag (prostaglandins); macitentan (ERA); riociguat (soluble guanylate cyclase stimulator of the NO family). Treprostinil has a half-life longer than epoprostenol and can be administered subcutaneously and intravenously. For this drug to show efficacy comparable to epoprostenol, the dosing level 1.3 times to twice the level of epoprostenol is needed (varying among individual cases). Although the vasodilative potential is higher with epoprostenol, treprostinil is less likely to induce thrombocytopenia. For these reasons, switching epoprostenol to treprostinil has been attempted in cases showing marked adverse reactions (e.g., thrombocytopenia) to epoprostenol despite improvement seen in response to epoprostenol treatment. When treprostinil is administered subcutaneously, local pain needs to be controlled with an analgesic.

Iloprost is the only prostaglandin $\mathrm{I}_{2}\left(\mathrm{PGI}_{2}\right)$ derivative for inhalational use among the pulmonary vasodilators available at present. It is a preparation for inhalation by the patient using a portable nebulizer. It is to be inhaled 6-9 times a day at intervals of 2 hours or more. Because this is an inhalational drug preparation, there is a limitation in dosing level (i.e., in the efficacy). However, unlike the preparations for intravenous or subcutaneous injection, this preparation is simple to administer.

Selexipag is a drug first demonstrated as effective in a global-scale prospective study among the oral-dose pulmonary vasodilators of the prostaglandin family. Although having a chemical structure (non-prostanoid structure) differing from conventionally available drugs (PGI 2 or its analogs), this drug stimulates the $\mathrm{PGI}_{2}$ receptor. In the GRIPHON study, a prospective double-blind study involving 1,156 patients with IPAH, symptom aggravation/ death (primary endpoint) was reduced by $40 \%$ (incidence: $27.0 \%$ in the selexipag group vs $41.6 \%$ in the placebo group). ${ }^{137}$

Macitentan is the third ERA introduced in Japan (marketed in 2015). It is less likely to cause adverse reactions and reported to have high efficacy. Its structure is a modification of the bosentan's structure and can be characterized by high tissue transfer and affinity. In a prospective clinical study adopting clinical aggravation as a primary endpoint (different from the design of conventional clinical trials of pulmonary vasodilators), the macitentan $10 \mathrm{mg}$ treatment group showed significant improvement compared to the placebo group and the macitentan $3 \mathrm{mg}$ treatment group. ${ }^{138}$

Riociguat is a drug exerting vasodilative activity by stimulating guanylate cyclase and increasing cyclic guanosine monophosphate (cGMP). Its clinical efficacy was confirmed in PATENT-1 and PATENT-2 studies. ${ }^{139,140}$ In the PATENT-1 study, 443 patients were allocated to the placebo group and the drug group (treated with riociguat $2.5 \mathrm{mg}$ three times daily). Three months after the start of treatment, the drug treatment group showed a significant increase in $6 \mathrm{MWD}$ to $30 \mathrm{~m}$, accompanied by significant improvement or alleviation of pulmonary vascular resistance (PVR), BNP precursor N-terminal fragment (NT- 


\begin{tabular}{|c|c|c|c|}
\hline & & Contraindicated & Caution needed \\
\hline \multirow{3}{*}{$\begin{array}{l}\text { Endothelin } \\
\text { receptor } \\
\text { antagonists }\end{array}$} & Ambrisentan & None & Cyclosporine \\
\hline & Bosentan & $\begin{array}{l}\text { Cyclosporine } \\
\text { Tacrolimus } \\
\text { Glibenclamide }\end{array}$ & $\begin{array}{l}\text { HMG-CoA reductase inhibitors } \\
\text { Warfarin } \\
\text { Oral contraceptives } \\
\text { Rifampicin } \\
\text { Ketoconazole, fluconazole } \\
\text { PDE5 inhibitors } \\
\text { Anti-HIV drugs }\end{array}$ \\
\hline & Macitentan & $\begin{array}{l}\text { Strong CYP3A inducing agents (rifampicin, } \\
\text { carbamazepine, phenytoin, phenobarbital, } \\
\text { rifabutin) }\end{array}$ & $\begin{array}{l}\text { Strong CYP3A inhibitors (ketoconazole, anti-HIV drugs), } \\
\text { CYP3A inducing drugs (efabirenz, modanifil, rufin- } \\
\text { amide) }\end{array}$ \\
\hline \multirow{2}{*}{$\begin{array}{l}\text { PDE5 } \\
\text { inhibitors }\end{array}$} & Sildenafil & $\begin{array}{l}\text { Nitric acid preparations } \\
\text { CYP3A inhibitors (ritonavir, darunavir, indinavir, } \\
\text { itraconazole, amiodarone, telaprevir, } \\
\text { cobicistat-containing preparations) } \\
\text { sGC stimulators (riociguat) }\end{array}$ & $\begin{array}{l}\text { CYP3A4 inhibitors (saquinavir, erythromycin, cimetidine, } \\
\text { atazanavir, clarithromycin, telithromycin, nelfinavir) } \\
\text { CYP3A4-inducing drugs (dexamethasone, phenytoin, } \\
\text { rifampicin, carbamazepine, phenobarbital) } \\
\text { Bosentan } \\
\text { Hypotensors (amlodipine, metoprolol, enalapril, } \\
\text { candesartan) } \\
\text { a-blockers } \\
\text { Carperitide } \\
\text { Warfarin }\end{array}$ \\
\hline & Tadalafil & $\begin{array}{l}\text { Nitric acid preparations } \\
\text { Strong CYP3A4 inhibitors (itraconazole, ritonavir, } \\
\text { atazanavir, indinavir, nelfinavir, saquinavir, } \\
\text { darunavir, clarithromycin, telithromycin, } \\
\text { telaprevir) } \\
\text { CYP3A4-inducing drugs (phenytoin, rifampicin, } \\
\text { carbamazepine, phenobarbital) } \\
\text { sGC stimulators (riociguat) }\end{array}$ & $\begin{array}{l}\text { CYP3A4 inhibitors (fosamprenavir, diltiazem, } \\
\text { erythromycin, fluconazole, verapamil) } \\
\text { Hypotensors (amlodipine, metoprolol, enalapril, } \\
\quad \text { candesartan) } \\
\text { Bosentan } \\
\text { CYP3A4-inducing drugs } \\
\text { a-blockers } \\
\text { Carperitide } \\
\text { Warfarin }\end{array}$ \\
\hline
\end{tabular}

proBNP), NYHA/WHO functional class, time until aggravation, and Borg scale. In the PATENT-2 study (an open-label extension study after PATENT-1), 396 patients were allocated to the drug treatment group, resulting in a mean increase in 6MWD by $51 \mathrm{~m}$ and improvement in the NYHA/WHO class seen in $33 \%$ of all patients one year after the start of treatment.

In recent years, discussions were made about how the goal of IPAH/HPAH treatment should be set. According to the ESC/ERA Guidelines for the Diagnosis and Treatment of Pulmonary Hypertension 2015 and other publications, there is a view in Western countries that the prognosis can be improved by achieving the goal of treatment set as "improvement noted as stabilization and satisfaction" on the basis of a general assessment of prognostic factors, ${ }^{141}$ and under this view it is often avoided to set normalization of pulmonary hemodynamics as an absolute goal of treatment. In Japan, on the other hand, there is a prevailing view that normalization of pulmonary hemodynamics should be set as a goal of treatment, and it has been recommended to perform dose increase and combined use of drugs for treatment, resulting in improvement of prognosis as a result of treatment under such a strategy..$^{\mathbf{9}}$ However, in cases of IPAH/HPAH, normalization of pulmonary hemodynamics by comprehensive treatment has been achieved in some cases, but it is often quite difficult to achieve in cases of CHD-PAH such as Eisenmenger's syndrome. Many of the clinical studies described here involved patients with IPAH/HPAH among Group 1 (PAH) cases, and the number of patients with CTD-PAH, or CHD-PAH participating in the studies was not large. It was recently reported that the etiology, pathophysiology, clinical features, and prognosis varied greatly among diseases of the same Group 1 (PAH) such as IPAH/HPAH, CTD-PAH, and CHD-PAH. ${ }^{138}$ We may therefore IPAH/ HPAH treatment strategy is not equally applicable to all types of PAH and that the treatment of each type of PAH should be designed in a manner tailored to the individual. The step of assessing the disease characteristics of each type of PAH and setting the goal of treatment are assigned at present to individual attending physicians. For this reason, the therapeutic strategy should be decided at facilities with sufficient experience.

\section{d. Combination Therapy}

There are many cases in which satisfactory responses to treatment cannot be obtained with treatment with a single drug. Combination therapy with 2 or 3 drugs with different action mechanisms is now extensively performed. According to a report from a facility with specialists in Japan, 103 $(79.2 \%)$ of the 141 patients with IPAH/HPAH who had been started their treatment before August 2012 received combination therapy, and their 5-year survival rate was $85.8 \% .98$

Combination therapy for PAH includes sequential combination therapy (sequential addition of pulmonary vasodilators to achieve the treatment goal) and upfront combination therapy (use of a combination of multiple drugs from the early stage of treatment with little time lag). In the first study of sequential combination therapy, bosentan was used as the initial drug and sildenafil and iloprost (inhalational, intravenous) were added sequentially as needed, but the 3-year survival rate did not increase over $79.9 \%,{ }^{143}$ indicating that the outcome of this therapy was 
not superior to the outcome reported in Japan.

Evidence for upfront combination therapy was provided by the AMBITION study using ambrisentan and tadalafil. The upfront combination therapy using these two drugs reduced the risk of death/PAH aggravation and hospitalization/disease progression (the composite endpoint) by $50 \%$ compared to each mono treatment. ${ }^{144}$ There is also a report that the upfront combination therapy with three drugs (bosentan, sildenafil, and epoprostenol) improved the hemodynamics and exercise tolerance of 19 patients with IPAH/HPAH whose NYHA/WHO Class IV, allowing achievement of a $100 \%$ three-year survival rate. ${ }^{145}$ In Japan, where the regulation of treatment methods under health insurance is minimal, it may be better to select upfront combination therapy rather than sequential combination therapy. However, since the evidence available at present is limited to the extent mentioned above, there is not sufficient reference information when deciding which one of multiple drugs with identical action mechanisms should be selected or how many kinds of drug should be selected. Because treatment of this disease uses multiple high-cost drugs, it is necessary to ask opinion at wellexperienced facilities.

\section{e. Drug Interactions}

Pulmonary vasodilators are often used in combination with other pulmonary vasodilators or drugs of other categories. Some of the pulmonary vasodilators are degraded by enzymes, such as CYP or induce drug-degrading enzymes, and there are known interactions among these drugs. Sufficient care is needed when using EPA ${ }^{146}$ and PDE5 inhibitors (closely involved in drug-degrading enzymes) for combination therapy.

Bosentan $^{147}$ is a derivative of CYP3A4 and CYP2C9. When this drug is used in combination with drugs degraded by these enzymes, the blood level of these drugs can decrease. Furthermore, since bosentan is degraded by CYP3A4 and CYP2C9, its use in combination with drugs degraded by these enzymes can lead to higher bosentan levels. For example, when used in combination with bosentan, sildenafil149,150 (a PDE5 inhibitor degraded by CYP3A $4{ }^{148}$ ) can show a decrease in blood level, requiring care of possible clinical aggravation due to poor drug efficacy. Furthermore, when used in combination with sildenafil, bosentan can exhibit a higher blood concentration. ${ }^{151,152}$ Tadalafil ${ }^{153}$ is also degraded by CYP3A4 and its blood level is reduced by combined use of bosentan, but the interaction is less than that seen following combined bosentan+sildenafil treatment. It is relatively rare that ambrisentan (an ERA) ${ }^{\mathbf{1 5 4}}$ affects the drugs that are the substrates for these enzymes. ${ }^{\mathbf{1 5 5 , 1 5 6}}$

Because sildenafil ${ }^{157}$ is degraded primarily by CYP3A4, drug interactions mediated by CYP3A4 can occur if used in combination with other drugs. Drugs inhibiting CYP3A4, such as erythromycin and cimetidine, can increase the blood sildenafil level. Tadalafil is also degraded by CYP3A4, hence requiring caution when used in combination with drugs involved in degradation by CYP3A4. Riociguat, an sGC stimulator, ${ }^{\mathbf{1 5 8 , 1 5 9}}$ has been reported to induce adverse reactions, such as hypotension and syncope, when used in combination with PDE5 inhibitors, and it is contraindicated to use riocinguat in combination with PDE5 inhibitors. Table 12 summarizes the drugs contraindicated for combined use and drugs requiring caution in combined use, with emphasis on ERA and PDE5 inhibitors. ${ }^{1}$

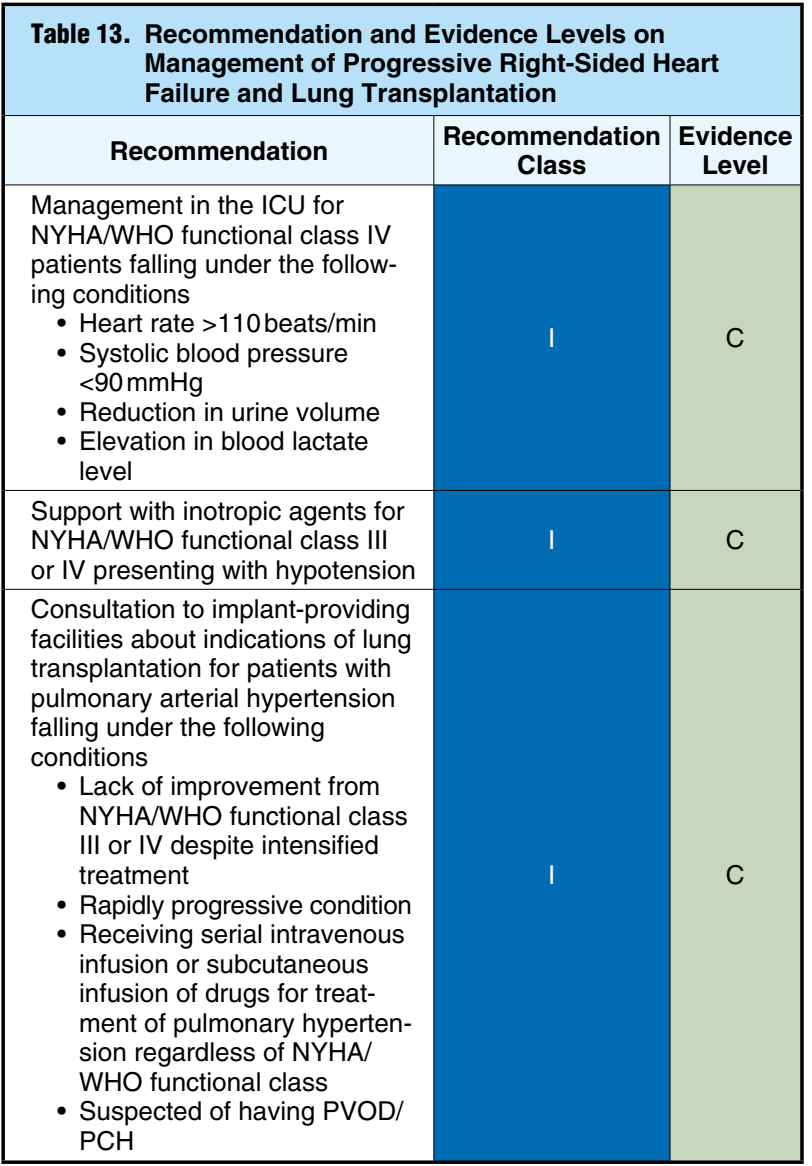

\section{f. Management of Progressive Right-Sided Heart Failure i. Management in the ICU}

In cases in which right-sided heart failure has advanced severely, management in the ICU should be considered. According to the ESC/ERS Guidelines for the Diagnosis and Treatment of Pulmonary Hypertension 2015, management in the ICU is recommended if the patient at the WHO Functional Class IV has reached the following conditions. ${ }^{1}$ A similar recommendation is made also in these guidelines (Table 13).

- Heart rate $>110$ beats $/ \mathrm{min}$

- Systolic blood pressure $<90 \mathrm{mmHg}$

- Reduction in urine volume

- Elevation in blood lactate level

Patients at WHO functional class III or IV presenting with hypotension should be supported by inotropic agents.

The goal of ICU management should be to achieve a heart rate and systolic blood pressure not falling under any of the above-described ICU admission criteria, accompanied by maintaining sufficient urine volume and achieving central venous oxygen saturation $\left(\mathrm{ScvO}_{2}\right)>70 \%$ or mixed venous oxygen saturation $\left(\mathrm{SvO}_{2}\right)>65 \%$ and blood lactate level $<2.0 \mathrm{mmol} / \mathrm{L} .{ }^{160}$ Because the prognosis of $\mathrm{PAH}$ patients is known to be poor if the right atrial pressure (RAP) is $15 \mathrm{mmHg}$ or higher, diuretics are used as needed to achieve RAP $\leq 14 \mathrm{mmHg}$ and control of bodily fluid volume. Volumetric loading should be avoided in patients with increased RAP because it can cause further right ventricular dilation and a shift of the ventricular septum to the left ventricle, resulting in further deterioration of right- 


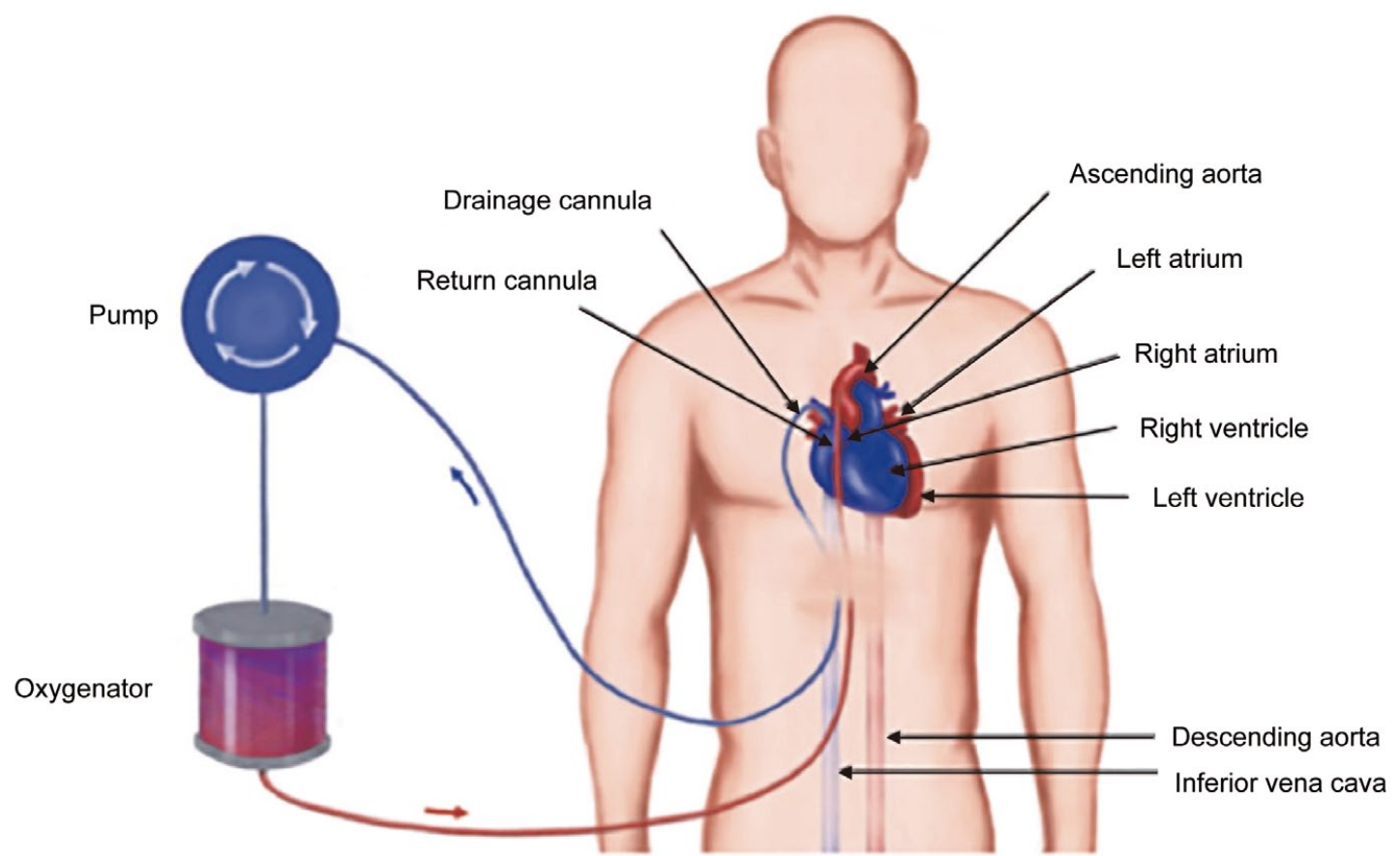

Figure 3. Diagram of central ECMO. Under thoracotomy, the blood is drained via the right atrium and transferred through the pump and the artificial lung into the ascending aorta. (Source: Pavlushkov E, et al. 2017166)

sided heart function and even impaired left ventricular filling. As an inotropic agent, dobutamine is usually used. However, in cases in which tachycardia is aggravated by dobutamine, phosphodiesterase-3 (PDE3) inhibitors (milrinone, olprinone) are sometimes used. PDE3 inhibitors have pulmonary vasodilator effects and can therefore reduce the right ventricular afterload, but care is needed of the frequent need of combined use of vasopressors since they reduce systemic vascular resistance. Dopamine and noradrenaline are primarily used as vasopressors. Although noradrenalin and high-dose dopamine can increase PVR, preservation of systemic blood pressure is important in maintaining blood flow and cardiac function. Vasopressin can increase systemic blood pressure without causing PVR elevation and is sometimes used instead of noradrenaline. However, the experience with its use in PAH patients is insufficient, ${ }^{161-163}$ thus requiring careful judgment as to its use. The accompanying diseases covered by the recommendation and are factors aggravating right-sided heart failure (anemia, infection, arrhythmia) also need to be treated appropriately. Hypoxemia, hypercapnia, and acidosis need to be corrected as much as possible since they can cause PVR elevation. Intrathoracic pressure elevation should also be avoided since it can aggravate pulmonary blood flow. Tracheal intubation should be avoided as much as possible because it often causes collapse of hemodynamics. If it is performed for inevitable reasons, auxiliary circulation (described later) should be available before such a procedure.

\section{ii. Auxiliary Circulation}

In cases in which circulation has failed, the use of venoarterial extracorporeal membrane oxygenation (V-A
ECMO) should also be considered. Veno-venous (V-V) ECMO is usually not performed because it does not lead to reduction of right ventricular loads. The purpose of ECMO is to provide either a bridge to recovery (BTR) or bridge to transplantation (BTT). In Japan, where the waiting period until transplantation is quite long, the use of ECMO as BTT is not realistic, but this should be considered for patients ranked high on the waiting list, considering the overseas report of cases in which transplantation was performed 30 days or more after ECMO support. ${ }^{164}$ Furthermore, since PAH is basically a chronic and progressive disease, patients can be seldom weaned from the ECMO. However, weaning from ECMO is sometimes possible in cases in which the accompanying disease, which is the aggravating factor for right-sided heart failure, has become treatable or cases in which drug therapy for PAH has failed to show satisfactory efficacy, ${ }^{165}$ and administration of ECMO should be considered in such cases.

Peripheral ECMO, which is a conventional type of ECMO with an approach from the inguinal region, involves problems such as leg ischemia, insufficient flow rate, bleeding from puncture site, infection, central hypoxia, and increased left ventricular after loads. Central hypoxia is a condition in which due to poor oxygenation by the patient's own lungs, blood without sufficient oxygenation is released from the left ventricle, entering the coronary artery and the cervical branches. Furthermore, since peripheral ECMO causes an increase in left ventricular after loads, lung edema may be induced, leading to central hypoxia. In such cases, central ECMO, which is conducted under thoracotomy by removing blood from the right atrium and transferring it to the ascending aorta, is used (Figure 3). ${ }^{166}$ If a left ventricular apical vent (for guiding 
blood out) is used simultaneously, the left ventricular afterloads can be reduced. Its use should be considered in cases in which lung edema may develop after ECMO administration.

\section{g. Lung Transplantation}

Lung transplantation may be indicated in NYHA/WHO functional class III/IV cases failing to respond to every medical treatment. The age for bilateral lung transplantation is less than 55 years at the time of registration and that for unilateral lung transplantation is less than 60 years at the time of registration, as a rule. In cases of pulmonary hypertension, bilateral lung transplantation is usually needed to ensure a sufficient lung vascular bed and ages less than 55 may be considered as indicated. As of the end of 2017, brain-dead donor lung transplantation has become possible at 10 facilities (facilities certified for lung transplantation) in Japan, including Okayama University, Kyoto University, Osaka University, Tohoku University*, National Cerebral and Cardiovascular Center**, Dokkyo Medical University, Fukuoka University, Nagasaki University, Chiba University, and University of Tokyo (in the order of the year of certification) (*simultaneous heartlung transplantation possible, **only simultaneous heartlung transplantation possible). Patients are enrolled on the lung transplantation waiting list in the following steps. First, the indications for lung transplantation are assessed at the facility certified for lung transplantation. This is followed by review at the Central Lung Transplantation Indications Review Committee and, if approved at the Committee, the patient is registered with the Japan Organ Transplantation Network. In cases in which registration with the waiting list is considered, consultation is first sought at the facility providing brain-dead donor lung transplantation. According to the guidelines prepared by the International Society for Heart \& Lung Transplantation, consultation should be considered in the following cases $^{\mathbf{1 6 7}}$ (Table 13).

- Lack of improvement from NYHA/WHO functional class III or IV despite intensified treatment

- Rapidly progressive condition.

- Receiving serial intravenous infusion or subcutaneous infusion of drugs for treatment of pulmonary hypertension regardless of NYHA/WHO functional class

- Suspected of having PVOD/PCH

According to the same guidelines, registration with the waiting list is recommended for the following cases.

- Lack of improvement from NYHA/WHO Functional Class III/IV despite at least 3-month combination therapy including non-oral prostanoid

- Cardiac index $<2 \mathrm{~L} / \mathrm{min} / \mathrm{m}^{2}$

- Mean RAP > $15 \mathrm{mmHg}$

- 6MWD $<350 \mathrm{~m}$

- Aggravation of hemoptysis, pericardial effusion, increased signs of right-sided heart failure (renal impairment, bilirubin increased, BNP increased, intractable ascites).

For live donor lung transplantation, registration with the Japan Organ Transplant Network is not indispensable. However, because the Guidelines on Live Donor Partial Lung Transplant prepared by the Japan Society for Transplantation recommend to implement this kind of transplantation at facilities providing brain-dead donor lung transplantation, it is advisable to consult a brain-dead donor lung transplantation providing facility also when live donor lung transplantation is considered.
As of end-January 2017, 309 patients were on the lung transplantation waiting list and $76(24.6 \%)$ of these patients had pulmonary hypertension as the underlying disease (the most frequent underlying disease). As of the end of 2015, the mean waiting period for the 283 patients on the lung transplantation waiting list was long, being 1,164 days when calculated including the patients whose registration was temporarily suspended (OFF cases showing improvement or stabilization of the condition in response to medical or other treatment) and 743 days when calculated only for the registration $\mathrm{ON}$ cases. Of the total of 1,019 registered patients, including patients with underlying disease other than pulmonary hypertension, 391 patients $(38.4 \%)$ died during the waiting period. In view of such a current status, it is necessary that patients for whom lung transplantation should be considered are enrolled on the waiting list as soon as possible.

According to the report by the International Society for Heart \& Lung Transplantation in 2016, ${ }^{168} 1,435$ (2.9\%) of the 50,002 patients having undergone lung transplantation were cases of IPAH. Most of these patients had undergone brain-dead donor bilateral lung transplantation, with the perioperative mortality rate being about $20 \%$ and the five-year survival period being about 55\%. In Japan, 535 patients underwent lung transplantation by the end of January 2017, including 75 cases of IPAH (14\%). The operative procedure for IPAH cases was brain-dead donor bilateral lung transplantation in 48 cases and live donor lung transplantation in 27 cases. The perioperative mortality rate $(13.3 \%)$ and the five-year survival rate $(82.4 \%)$ were favorable.

\section{h. Cardiopulmonary Transplantation}

\section{i. Criteria for Indications}

The indications established by the Cardiopulmonary Transplantation Indications Review Committee of the Japanese Circulation Society are: (1) Lung disease indicated for lung transplantation including pulmonary hypertension accompanied by compromised cardiac function, (2) congenital heart disease accompanied by pulmonary hypertension (Eisenmenger's syndrome) difficult for surgical repair or accompanied by compromised cardiac function, (3) congenital heart disease accompanied by pulmonary hypoplasia difficult for surgical repair or accompanied by compromised cardiac function, and (4) other conditions acknowledged by the review committee.

The requirements to be satisfied for application of cardiopulmonary transplantation include: (1) cases of advanced lung disease possibly indicated for lung transplantation, accompanied by congenital heart disease or severely compromised cardiac function difficult for surgical repair and unable to resolve clinical symptoms (corresponding to NYHA Class III/IV) despite maximum medical treatment, (2) cases of severe heart failure possibly indicated for heart transplantation, accompanied by drug-resistant irreversible pulmonary hypertension (transpulmonary gradient [TPG] $\geq 15 \mathrm{mmHg}$ or PVR $\geq 8.0$ Wood units following nitrogen monoxide inhalation or intravenous prostacyclin injection), (3) age $\leq 55$, and (4) sufficient understanding and cooperation of the patient and family members about/with cardiopulmonary transplantation. In cases of Eisenmenger's syndrome, transplantation is considered if frequent massive hemoptysis or drug-resistant ventricular arrhythmia/cardiac impairment becomes apparent. ${ }^{169}$

Patients with preserved cardiac function and correctable 
congenital heart disease are indicated for bilateral or unilateral lung transplantation combined with surgical repair of congenital heart disease. Cardiopulmonary transplantation is indicated in cases of complex heart malformation (e.g., double outlet right ventricle, complete transposition of great arteries) anticipated to undergo one-hour or longer aortic block during intracardiac repair or cases of congenital heart disease allowing only Fontan operation (e.g., univentricular heart). However, cases of double outlet right ventricle accompanied by subaortic ventricular septal defect may be judged as indicated for bilateral/unilateral lung transplantation and intracardiac repair at the discretion of the surgeon. ${ }^{170}$ If the number of donated organs available is ignored, the criteria for indications of this surgery will alter over time, in view of the report that the prognosis of patients with Eisenmenger's syndrome has been reported to be better following cardiopulmonary transplantation than following lung transplantation even in patients with ventricular septal defect (VSD).

Absolute contraindications against cardiopulmonary transplantation include irreversible liver/kidney dysfunction, local/systemic infection, drug addition, malignant tumor, and positive HIV antibody test. Relative contraindications include reversible liver/kidney dysfunction, peptic ulcer, complicated insulin-dependent diabetes mellitus, marked thoracic deformation, and extensive pleural adhesion/scarring, marked neuromuscular disease, extreme malnutrition or obesity, patient unable to receive rehabilitation expected to have such ability, and critical psychosocial disorders preventing understanding/cooperation of patient or family members.

\section{ii. Donor-Recipient Compatibility and Selection of Recipients} Because the lungs are often damaged before brain death of the donor or resection of the lungs from the brain-dead donor, only about $1 / 4$ of all heart donors are suitable as the donors for cardiopulmonary transplantation. The acceptable safe storage period of cardiopulmonary grafts is known to be within 4 hours when simply stored by immersion, as is the case with heart grafts. Furthermore, the criteria for both the heart donors and the lung donors need to be satisfied by cardiopulmonary donors.

Patients desiring to receive cardiopulmonary transplantation are registered with both the heart transplantation list and the lung transplantation list. Patients selected as the first-ranked recipient with one of the recipient selection criteria for individual organs are selected as the candidate recipient.

\section{iii. Cardiopulmonary Transplantation in Japan}

In April 2003, the application for evaluation of the indications for cardiopulmonary transplantation began to be received by the Japanese Circulation Society. The indications include congenital heart disease (accompanied by Eisenmenger's syndrome), complex heart malformation (accompanied by pulmonary artery hypoplasia), restrictive cardiomyopathy (RCM) (accompanied by marked pulmonary vascular resistance, including left ventricular hypoplasia), and dilated cardiomyopathy. After that, cardiopulmonary transplantation began to be covered by national health insurance in April 2006 together with national health insurance coverage of heart transplantation, lung transplantation, and other transplantations, although no case had undergone this transplantation by that time. One patient with pulmonary hypertension accompanied by dilated cardiomyopathy is now alive after having undergone heart transplantation in Germany because of reduction in PVR. The presence of this case suggests that the diagnosis of reversibility of pulmonary hypertension originating from heart failure is not always easy.

In January 2009, the first cardiopulmonary transplantation in Japan was performed for a male in his $30 \mathrm{~s}$ with Eisenmenger's syndrome and double outlet right ventricle, and the second and third such operations were performed in December 2013 and June 2016, respectively, in patients with $\mathrm{RCM} /$ marked pulmonary hypertension (one male and one female in their $20 \mathrm{~s}$ ). As of 2017, all three of these patients are alive.

\section{i. Diagnosis and Management of Complications \\ i. Arrhythmia}

Arrhythmia is a significant clinical problem for $\mathrm{PAH}$ patients. Supraventricular tachycardia develops at a frequency of about $2.8 \%$ per year. ${ }^{171}$ The frequency of atrial flutter and atrial fibrillation is close to this rate, both triggering aggravation of right-sided heart failure. ${ }^{172}$ There is a report that maintenance of sinus rhythm is associated with long-term survival of PAH patients and that the two-year mortality rate of $\mathrm{PAH}$ patients complicated by persistent atrial fibrillation exceeded $80 \%{ }^{172}$ In another study that followed 231 patients with PAH or CTEPH for 6 years, fatal ventricular arrhythmia was not seen in any case. Thus, the frequency of ventricular tachycardia, ventricular flutter, and fatal ventricular arrhythmia, such as ventricular fibrillation, is quite low.

Atrial fibrillation is indicated for warfarin or DOAC treatment as anticoagulant therapy. In treatment-resistant cases, electrical defibrillation or catheter ablation should be considered, but there is no sufficient data supporting their efficacy in PAH patients. Also concerning the efficacy of antiarrhythmic drugs (such as oral-dose amiodarone), further accumulation of evidence is needed.

\section{ii. Hemoptysis}

Hemoptysis is a complication that can lead directly to death, with the incidence varying greatly (1-6\%) among reports. It is often associated with not only HPAH but also CHD-PAH and CTEPH. ${ }^{173}$

Bronchial artery embolization is an urgent measure to treat serious hemoptysis. Anticoagulant therapy should be avoided in patients presenting with hemoptysis.

\section{iii. Mechanical Complications}

In patients with advanced PAH, the pulmonary artery can be dilated by chronic exposure to high pressure. This can induce aneurysms, rupture, or dissection of pulmonary arteryies, and can mechanically compress the coronary artery, pulmonary vein, and recurrent nerve. ${ }^{174-176}$ These complications can lead to sudden death or various symptoms such as compression-caused chest pain and lung edema. Contrast-enhanced CT scan is recommended for diagnosing pulmonary aneurysm. As a rule, surgical treatment is performed, but there are no well-defined diagnostic criteria or standard treatment methods for pulmonary aneurysm. In cases presenting with symptoms caused by mechanical compression, lung transplantation or cardiopulmonary transplantation should also be considered. If anginal pain due to compression of the left coronary artery develops repeatedly, percutaneous stent therapy should also be considered. ${ }^{177}$ 


\section{iv. Indwelling Catheter Infection}

In patients with $\mathrm{PAH}$ receiving serial drip infusion of epoprostenol or treprostinil, infection via the indwelling catheter is a serious problem. In many cases, this kind of infection is detected with symptoms such as subcutaneous redness and pain caused by local infection of the catheterinvaded area. If it progresses to sepsis, there is a risk of death. Thus, early actions are essential. In a follow-up survey of 192 PAH patients, Staphylococcus aureus and Micrococcus spp. were isolated as the pathogens for sepsis at a rate of 0.15 cases $/ 1,000$ days of medication. ${ }^{178}$ Treatment with antibiotics is a basic approach. In patients developing abscess around the catheter or severe sepsis, debridement around the catheter and catheter withdrawal are needed. If subcutaneous infection has developed following subcutaneous treprostinil treatment, the puncture site is changed and antibiotics are administered. For prevention of this kind of infection, it is necessary to provide education to patients at the start of treatment and assess the catheter-created wounds by the outpatient care team primarily consisting of nurses.

\section{j. Terminal Care and Ethical Consideration}

Terminal care for cardiovascular disease is when the patient is facing imminent death despite continuation of appropriate healthcare. ${ }^{179}$ Due to advances in drugs specific to PAH treatment and other measures, the prognosis of PAH patients has improved markedly during the past approximately two decades, ${ }^{97}$ but $\mathrm{PAH}$ remains a disease with poor prognosis, involving major open issues such as how to treat the negative impact of terminal stage painful symptoms on the QOL of patients and how to provide palliative care.

PAH progresses with repeating cycles of aggravation (due to non-compensatory heart failure) and remission, eventually leading to the terminal stage by relatively rapid steps. There is also a risk of sudden death during the course of this disease, making accurate prediction of the outcome very difficult. ${ }^{1}$ For this reason, emphasis tends to be put on comprehensive treatment, including invasive treatment, and as a result the chance for discussion with the patient as to the desired manner of daily living during the terminal stage tends to be lost, accompanied by frequent loss of the timing for the patient to receive the benefit of palliative care. In practice, a report from the United States demonstrates that $52-83 \%$ of the PAH patients who died at medical facilities died while in the ICU. ${ }^{\mathbf{1 8 0 , 1 8 1}}$ According to the "Guidelines on the Process of Deciding Healthcare the Terminal Stage of Life" prepared by the Ministry of Health, Labour and Welfare, Japan, ${ }^{\mathbf{1 8 2}}$ healthcare and other care at the terminal stage of life should be based on the patient's own decision made by discussion with the healthcare providers, and its medical validity and appropriateness should be judged by a multidisciplinary team. It is therefore important that during introduction of palliative care, the course of PAH anticipated is shared by the healthcare team and the patient/family members in advance and the process of repeating dialogues is continues to allow the patient to make a choice best suited to his/her sense of value at the terminal stage (advance care planning: $\mathrm{ACP})$.

Palliative care is not synonymous with terminal care. Unlike the terminal care provided during the last several days to several weeks, palliative care is an approach aimed at alleviating the pain from early stages of care and main- taining/improving the QOL of the patient/family members. Pain should not be considered simply as physical pain but should be assessed also from mental, social, and spiritual aspects. ${ }^{183}$ Furthermore, the sense of value, the view of life, and the preferences of individual patients, which vary from individual to individual, should also be understood and respected. To this end, it is necessary to provide support during palliative care by a multidisciplinary team consisting primarily of physicians, nurses, and pharmacists. Because appropriate treatment itself can alleviate symptoms, treatment of PAH should be continued until the end. Effective communication between the healthcare provider and the patient is the base for palliative care, and it leads to the patient's deeper understanding of the disease knowledge, course of sickness, and awareness of prognosis. Opportunities for discussing prognosis should be arranged at the time of PAH diagnosis and at later appropriate points of time depending on the course of the disease, accompanied by explanation about treatment alternatives and risks involved. ${ }^{184}$ It is necessary to make efforts so that ACP is implemented in a manner allowing the patient to make the best possible choice, instead of focusing on the negative image about the prognosis. ${ }^{185}$ The survival prediction score, such as the REVEAL risk score, ${ }^{73}$ may be useful in judging the timing of palliative care intervention. In cases in which expert judgment is needed depending on the healthcare environments surrounding individual cases, it is necessary to seek consultation with a specialist in palliative care, but this kind of consultation is not covered by health insurance in Japan at present.

PAH causes marked symptoms during the terminal stage, markedly reducing the QOL of patients as seen in patients with chronic obstructive pulmonary disease (COPD), renal failure, and therapy-resistant cancer. ${ }^{186}$ Representative symptoms include dyspnea at rest and on exertion, malaise, tendency to fatigue, chest discomfort, lower leg edema, abdominal flatulence, and depression. In PAH patients at the terminal stage, dyspnea is the most frequent and severe symptom accompanied by the sensation of fear. When intervention is considered, not only physical factors but also psychological and social factors need to be taken into consideration. Low-dose opioids have been reported to alleviate therapy-resistant dyspnea in an effective and safe manner. ${ }^{187,188}$ Because adverse reactions to specific pulmonary vasodilators and problems, such as pain and infection arising from the drug administration route (central vein or subcutaneous tissue for injection), also markedly affect the QOL, ${ }^{\mathbf{1 8 9}}$ palliative care is useful also in achieving best care possible during the course of the disease. At the terminal stage, meal digestion sometimes decreases, but unnecessary intravenous fluid therapy can aggravate the symptoms of congestion, thus requiring caution before its application. PAH causes major mental and socioeconomic burdens on the patient and caregivers, ${ }^{190}$ with symptoms of anxiety reported to be experienced by about half of them and depressive symptoms in one-third. ${ }^{191}$ The ESC/ERS Guidelines for the Diagnosis \& Treatment of Pulmonary Hypertension 2015 recommend psychological/social support to PAH patients as Class I (Evidence Level C) recommendation, ${ }^{1}$ urging a system for providing such support or referral to an expert.

In cases in which complaint of intolerable pain is resistant to every treatment and death is expected within several days to a few weeks, sedation by medications, such as midazolam, should be considered. ${ }^{192}$ Sedation provided 

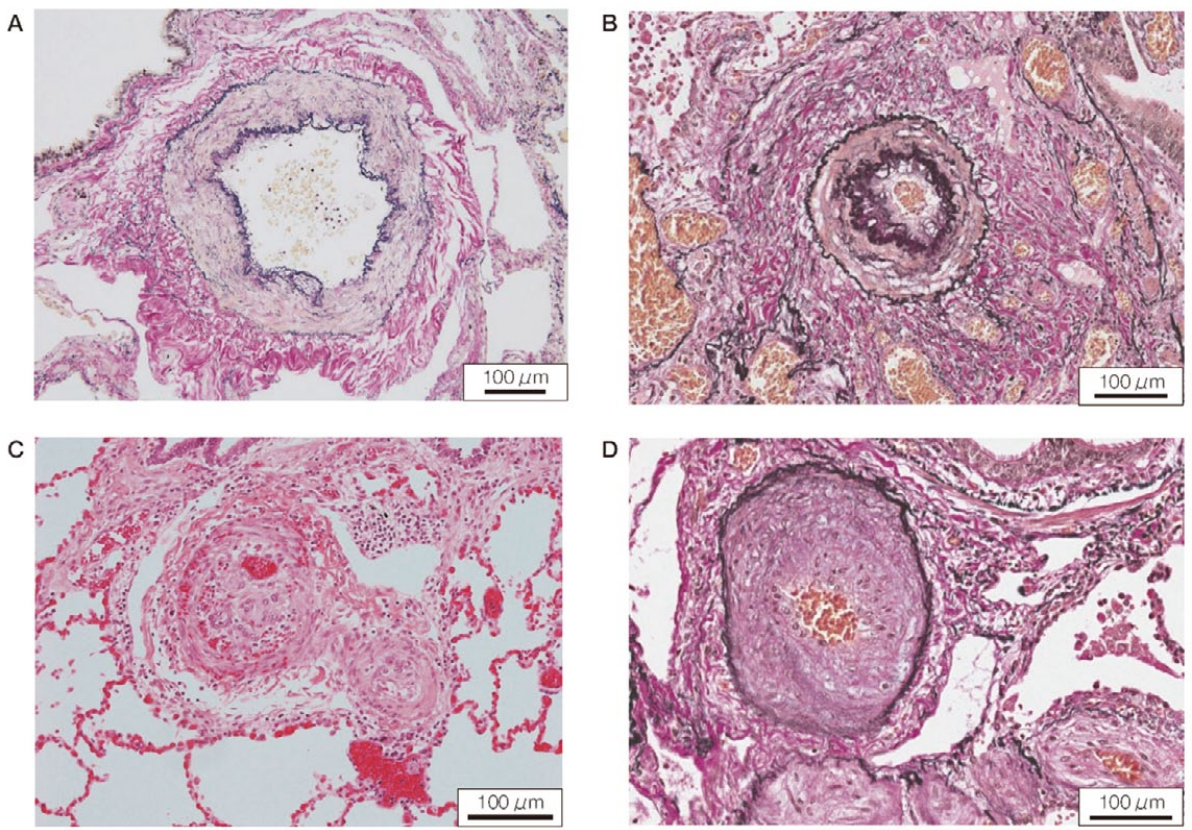

Figure 4. Reversible lesions of the pulmonary artery (isolated medial hypertrophy $[\mathbf{A}, \mathbf{B}]$ and a combination of medial hypertrophy and intimal thickening $[\mathbf{C}, \mathbf{D}])$. (A) Heath-Edwards grade 1. Hypertrophy of tunica media of muscular pulmonary artery (EVG staining). (B) Heath-Edwards grade 2. Hypertrophy of tunica media of muscular pulmonary artery accompanied by slight intimal thickening (EVG staining). (C) Heath-Edwards grade 3. Stenosis caused by medial hypertrophy accompanied by cellular intimal thickening (HE staining). (D) Heath-Edwards grade 3. Intimal thickening due to fibrosis as a primary element in addition to cellular elements (fibrocellular intimal thickening) (EVG staining).

under appropriate supervision does not shorten the survival period. ${ }^{193}$ Sedation needs to be provided carefully by a multidisciplinary team, on the basis of the wishes of the patient/family members, while avoiding overdose of medication or inappropriate medical measures, such as the use of muscle relaxant, which can shorten the survival period.

\section{k. Molecule-Targeted Therapy}

Treatment with 3 categories of drugs for PAH treatment (ERA, PGI2, and PDE5 inhibitors) markedly improved the prognosis of PAH patients. However, because the prognosis of advanced $\mathrm{PAH}$ remained poor despite treatment with these drugs, a new category of drugs targeting a different molecular mechanism has been developed.

Imatinib, a platelet-derived growth factor (PDGF) receptor antagonist, improves the pathologic tissue (i.e., tunica media hypertrophy) of animal models of hypoxiaand monocrotaline-induced disease, thus exerting excellent efficacy against pulmonary hypertension. ${ }^{194}$ In an international cooperative clinical study involving $202 \mathrm{PAH}$ patients including 25 Japanese (Imatinib in Pulmonary Arterial Hypertension, a Randomized, Efficacy Study; IMPRES), percent change in PVR at 24 weeks after the start of treatment aggravated to +12 dyne $\cdot \mathrm{sec} \cdot \mathrm{cm}^{-5}$ in the placebo group, but it improved significantly to -366 dyne $\cdot \mathrm{sec} \cdot \mathrm{cm}^{-5}$ in the imatinib group. There was no significant improvement in the NYHA/WHO functional class or survival rate, and epidural hematoma was reported as a serious complication in 8 patients having received imatinib and anticoagulant therapy. ${ }^{195}$ Following these results, the regulatory authority in the United States refused approval of the drug, pointing out: "It is difficult to judge that imatinib has appropriate risk-benefit balance when performed for PAH." Other than this drug, drugs such as vasoactive intestinal peptides, Rho kinase inhibitors, vascular endothelial growth factor receptor inhibitors, angiopoetin-1 inhibitors, and elastase inhibitors have been shown to be effective in many animal models, ${ }^{196}$ with their future clinical application for PAH patients promising.

\subsubsection{Pathology ${ }^{197,198}$}

In cases of PAH, it has been reported that morphological changes in the arterial wall can occur as histological phenotypes of lesions depending on the severity of pulmonary hypertension, instead of corresponding to the etiology of pulmonary hypertension. The lesions of PAH commonly appear in small pulmonary arteries with a diameter $500 \mu \mathrm{m}$ or less. If PAH persists, arteriosclerotic atheroma can develop also in the main pulmonary artery. For pathological evaluation of pulmonary hypertension, the Grades 1 to 6 of the Heath-Edwards grading system are often used although this is a classical grading system. ${ }^{68}$ Grades 1 to 3 lesions are known to be reversible by treatment of pulmonary hypertension, whereas Grades 4 to 6 differ little in terms of clinical severity and lesions of several grades occasionally coexist.

\section{a. Isolated Medial Hypertrophy}

(Medial hypertrophy: Heath-Edwards grade 1 (Figure 4A), medial hypertrophy accompanied by cellular intimal growth: corresponding to grade 2 (Figure 4B))

In the muscular arteries with a diameter up to 300 

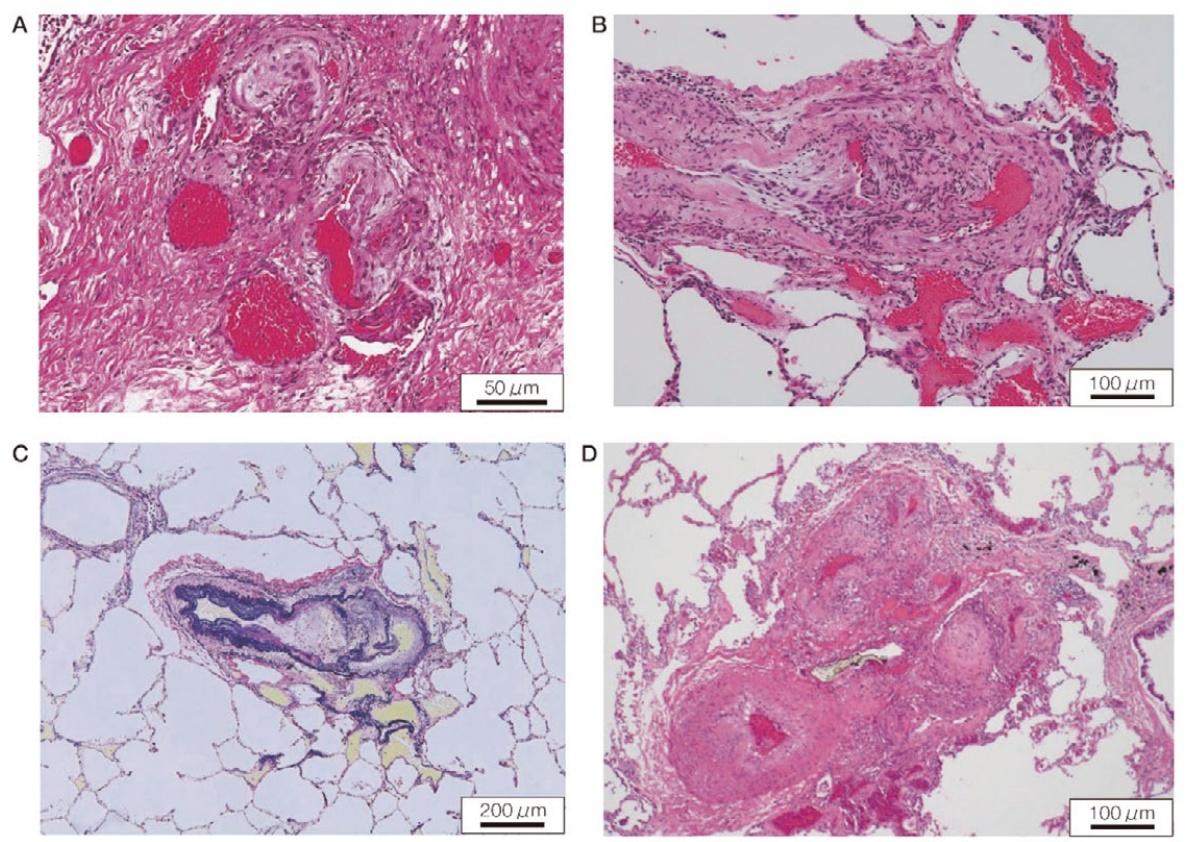

Figure 5. Composite vascular lesion. (A,B) Plexiform lesion (Heath-Edwards grade 4); Aneurysm-like or angioma-like branching from the main trunk of pulmonary artery accompanied by glomeruli-like vascular growth at the periphery or vascular dilatation around the tunica externa (HE staining). (C) Dilatation lesion (Heath-Edwards grade 5); Aneurysmal dilatation of capillaries around the abnormal branches of the artery (EVG staining). (D) Arteritis (Heath-Edwards grade 6); Inflammatory cell infiltration of pulmonary artery wall resulting in partial wall destruction (HE staining).

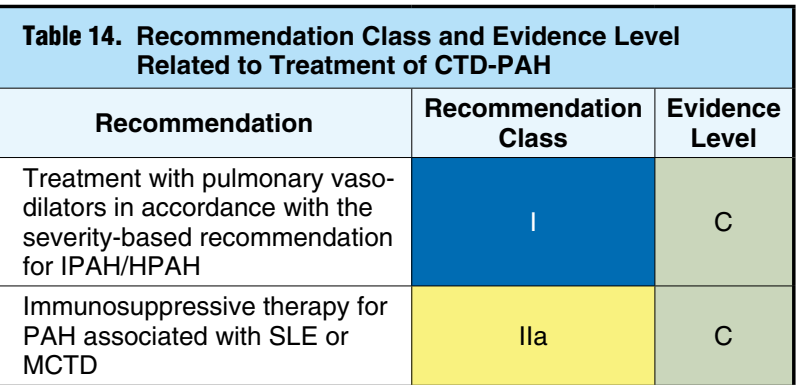

$500 \mu \mathrm{m}$, increases in pulmonary arterial pressure causes hypertrophy of medial smooth muscle cells (SMCs) and hypertrophy of the pulmonary artery medial wall due to an increase in SMCs. Furthermore, muscular layers are formed even at the arteriolar level $(20-30 \mu \mathrm{m})$ at which hardly any smooth muscle layer is normally present (muscularization of arteriole). ${ }^{199}$ In the muscular arteries of diameter about $300-500 \mu \mathrm{m}$, medial hypertrophy is marked. This hypertrophy of the tunica media is considered to reflect vascular contraction/dilatation adapted to increases in the pulmonary arterial pressure and is reversible if pulmonary hypertension is alleviated.

\section{b. With Intimal Thickening and Medial Hypertrophy}

(Corresponding to Heath-Edwards grade 3 (Figure 4C, Figure 4D))

The above-mentioned medial hypertrophy of the pulmo- nary artery is accompanied by intimal thickening. Depending on the features of tunica intima involved, this change can be divided into: (1) intimal thickening due to an increase in cellular components, such as $\alpha$-actin positive SMCs and myofibroblasts (cellular intimal thickening), and (2) intimal thickening due to an increase in elastic fibers, collagen fibers, and extracellular matrix (fibrous intimal thickening). Cellular thickening is reversible if pulmonary hypertension is alleviated, whereas fibrous intimal thickening with cellular features having been lost does not completely disappear.

\section{c. Complex Lesion}

Histological changes seen in advanced case of pulmonary hypertension include not only the above-mentioned medial hypertrophy and intimal thickening but also the following lesions: (1) plexiform lesion (Heath-Edwards grade 4, (2) dilatation lesion (grade 5), (3) and arteritis (grade 6, occasionally accompanied by fibrinoid necrosis). These lesions often appear in combination, rather than separately. In addition, the plexiform lesion is known to be irreversible.

(1) Plexiform lesion: A lesion branching approximately rectangularly from the main trunk of the muscular pulmonary artery at the periphery, with growth of capillaries similar to renal glomeruli in the knob-shaped blood vessel (Figure 5A, Figure 5B). This is also called an "angioma-like lesion" and is considered as representing the outcome of a pulmonary artery-vein shunt. Cells constituting the plexiform lesion include endothelial cells, smooth muscle cells, and fibroblasts. The plexiform lesion appears in various diseases, including 
IPAH, PoPH, PAH associated with CTD and PAH associated with HIV infection.

(2) Dilatation lesion: This indicates a blood vessel with a dilated and tortuous vein-like arrangement. This is often seen distal to the plexiform lesion. This type of lesion is also considered as a result of pulmonary artery-vein shunt (Figure 5B, Figure 5C).

(3) Arteritis: This often develops together with the plexiform lesion or the dilatation lesion. It is considered as a lesion occurring before the plexiform lesion. However, arteritis is occasionally seen also in CTD-PAH, and it also may be possible that other composite lesions can develop after the onset of arteritis (Figure 5D).

\section{I1.2 Pulmonary Arterial Hypertension Associated With Connective Tissue Disease (CTD-PAH)}

\section{Summary}

Patients with connective tissue disease (CTD) have a higher risk for developing pulmonary hypertension compared to the general population. The incidence of pulmonary hypertension is particularly high $(2-10 \%)$ in patients with systemic sclerosis (SSc), mixed connective tissue disease (MCTD) or systemic lupus erythematosus (SLE). Pulmonary hypertension of diverse clinical classifications is seen in patients with CTD, including not only PAH but also PVOD, pulmonary hypertension due to left heart disease, pulmonary hypertension due to lung disease (e.g., interstitial lung disease), CTEPH, pulmonary hypertension due to pulmonary arteritis, and some patients have a mixed form of these classifications of pulmonary hypertension. For early detection, screening by transthoracic echocardiography is recommended in patients with CTD at risk for PAH. Comprehensive evaluation about the clinical classification of pulmonary hypertension is essential before treatment is begun.

Both supportive therapy and pulmonary vasodilator therapy for CTD-PAH are performed in a manner similar to the therapeutic strategy for IPAH/HPAH. Immunosuppressive therapy is sometimes effective in cases of PAH due to SLE, MCTD, or Sjōgren's syndrome. In patients with the mixed form of pulmonary hypertension due to SSc, pulmonary vasodilators need to be used carefully. Although the survival of patients with CTD-PAH has been improved by use of pulmonary vasodilators, it is still poor compared to that of patients with IPAH/HPAH. Selection of optimum therapy tailored to individual cases is necessary.

The recommendation class and evidence level related to the treatment of CTD-PAH are shown in Table 14.

\subsubsection{Epidemiology and Etiology \\ a. Prevalence of Pulmonary Hypertension Among Patients With CTD}

The prevalence of pulmonary hypertension is higher among patients with CTD than among the general population. ${ }^{200}$ According to the survey conducted by "the Mixed Connective Tissue Disease Panel of the Skin/Connective Tissue Survey and Study Group under the Ministry of Health and Welfare Specific Disease Study Program" in 1998 (a survey covering only moderate to severe cases), the prevalence of pulmonary hypertension was $7.0 \%$ in MCTD, $5.0 \%$ in SSc, and $1.7 \%$ in SLE, and pulmonary hypertension was absent in polymyositis (PM) or dermatomyositis (DM). ${ }^{201}$ According to reports in which evaluation by right heart catheterization was indispensable, the prevalence of $\mathrm{PAH}$ among patients with SSc was $7-12 \% \mathbf{1 2 , 2 0 2 , 2 0 3}$ and was $9 \%$ in the meta-analysis of data from 3,818 patients. ${ }^{204}$ The prevalence among patients with SLE is low (about 2-4\%). ${ }^{205,206}$ Although no report has been published concerning evaluation of MCTD patients by right heart catheterization, the above-mentioned Japanese survey data in $1998^{201}$ suggest a prevalence comparable to or slightly higher than that among SSc patients. $\mathrm{PAH}$ is seen also in patients with primary Sjōgren's syndrome, although the actual prevalence is unknown. ${ }^{207}$

The distribution of underlying disease among the patients diagnosed as having CTD-PAH differs between Western countries and Japan. In Western countries, SSc is the underlying disease in more than $60 \%$ of all cases, ${ }^{87,208}$ whereas SSc, SLE, and MTCD account for an approximately same share (more than $90 \%$ in total) among patients with CTD-PAH according to the reports in Japan. ${ }^{209}$

\section{b. Clinical Classification of Pulmonary Hypertension Associated With CTD}

Diverse clinical classifications of pulmonary hypertension can occur in patients with CTD, ranging from PAH to PVOD (Group 1'), pulmonary hypertension due to left heart disease (Group 2), pulmonary hypertension due to lung diseases such as interstitial lung disease (ILD) (Group 3), CTEPH (Group 4), pulmonary hypertension due to pulmonary arteritis (Group 5). 208,210-212 Furthermore, a mixture of these clinical classifications is also seen frequently. Pulmonary hypertension observed in patients with SSc are classified as not only PAH but also pulmonary hypertension due to left heart disease and pulmonary hypertension due to ILD. ${ }^{211,212}$

\section{1.2.2 Evaluation of Prognosis/Severity a. Prognosis}

The survival of patients with CTD-PAH is poor compared to patients with IPAH/HPAH, ${ }^{208}$ and this trend remains unchanged also in recent years despite extensive application of combination therapy of pulmonary vasodilators. ${ }^{213,214}$ When analyzed by underlying disease, the prognosis is poorer for patients with SSc than for patients with SLE or MCTD. ${ }^{208,215}$

\section{b. Severity Scale}

Although there is no severity scale specific for CTD-PAH, a severity classification for pulmonary hypertension associated with SSc was proposed in the "Systemic Scleroderma Diagnosis Criteria/Severity Classification/Clinical Management Guidelines" prepared by the Ministry of Health, Labour and Welfare Scientific Study Program in 2016.216 This scale is based on the NYHA/WHO functional class. Because patients with CTD have various factors that can cause dyspnea on exertion (not only ILD and heart disease but also musculoskeletal disorders, and anemia), these factors need to be taken into consideration during functional evaluation. Numerous cohort studies have demonstrated that the underlying $\mathrm{SSc}$ and NYHA/WHO functional class III/IV are poor prognostic factors. ${ }^{209,217}$ Studies of patients with PAH associated with SSc (SSc$\mathrm{PAH}$ ) revealed hemodynamic indicators, such as cardiac index (CI), stroke volume index (SVI) and PVR, as factors for predicting outcome, similar to their roles in patients with IPAH. ${ }^{218,219}$ Older age and male are also poor prognostic factors. ${ }^{218}$ Although there is a report showing lack of the association of autoantibodies with prognosis, ${ }^{220}$ 


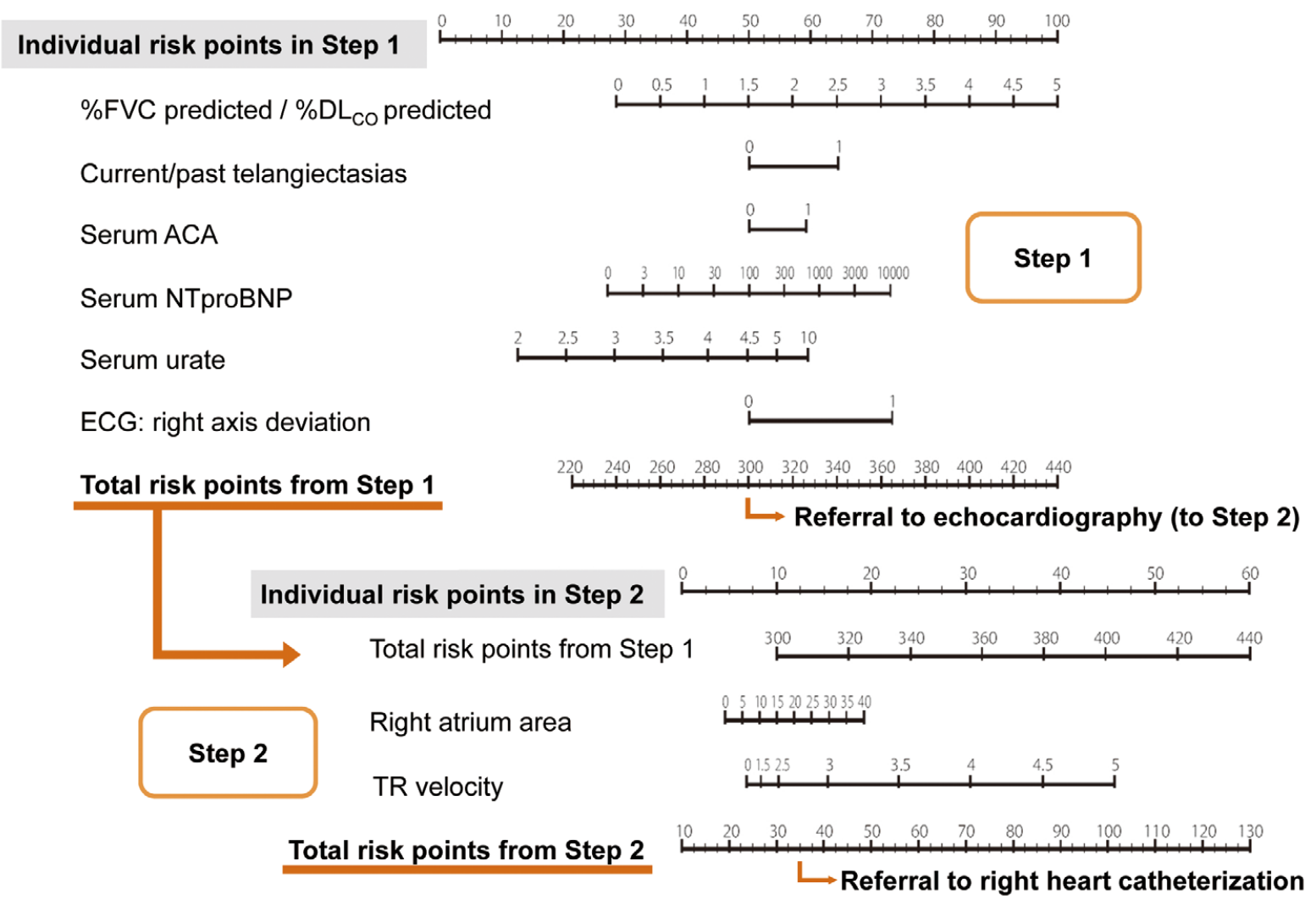

Figure 6. Two-step nomogram for selection of SSc patients indicated for right heart catheterization (DETECT). Subjects are SSc patients aged 18 and above, with disease duration of 3 -year or longer and \%DLco $<60 \%$. If the total risk point- 1 score during Step 1 is 300 or higher, Step 1 is advanced to Step 2. If the total risk point-2 score after transthoracic echocardiography is 35 or higher, right heart catheterization is recommended. (Source: Coghlan JG, et al. 2014226)

favorable prognosis has been shown in anti-U1RNP antibody positive cases of SSc-PAH. ${ }^{221}$ In the survey of prognosis conducted by "the Mixed Connective Tissue Disease Panel of the Skin/Connective Tissue Survey and Study Group under the Ministry of Health and Welfare Specific Disease Study Program" in 1997, polyarthritis, increased myogenic enzyme levels, satisfaction of PM/DM classification criteria, and skin sclerosis were identified as independent factors associated with poor prognosis of PAH due to MCTD. ${ }^{222}$

\subsubsection{Diagnostic Procedure}

\section{a. Diagnosis of CTD}

For each type of CTD, there are international classification criteria or domestic diagnostic criteria for the intractable disease, and diagnosis are made with reference to such criteria. However, these criteria include exclusion criteria, and the specificity is about $90 \%$ at most, with false-positive cases sometimes seen. Some cases of CTD, such as early cases and mild cases, fail to satisfy these criteria. For these reasons, consultation with rheumatologists is recommended when making diagnosis of this disease.

\section{b. Risk Factors for PAH}

Risk factors for PAH vary depending on the underlying disease. Among patients with SSc, PAH is often seen in patients with long-lasting limited cutaneous SSc (lcSSc), and more than $50 \%$ of all SSc-PAH cases are positive for anticentromere antibody. ${ }^{223-226}$ PAH often develops after a long duration of SSc, mostly at older age (60 and over). Other risk factors include telangiectasia that often affects fingers and lips. 227,228 Positive anti-U1RNP antibody is a risk factor for PAH regardless of underlying disease. In patients with SLE or MCTD, PAH often develops or aggravates simultaneously with onset or increased disease activity of the underlying CTD, and the duration of CTD before onset of PAH is often short.

\section{c. Screening}

In CTD patients at high risk for PAH, annual screening should be performed regardless of the presence/absence of symptoms for the purpose of facilitating early detection of PAH. In practice, screening of SSc cases has been shown to increase the percentage of mild cases (NYHA/WHO functional class I/II) at the time of diagnosis of PAH and to improve the survival. ${ }^{217}$ The ESC/ERS Guidelines for the Diagnosis and Treatment of Pulmonary Hypertension 2015 recommend screening with a combination of TTE and biomarkers, such as pulmonary carbon monoxide diffusing capacity (DLco) and BNP, at least once year in patients with $\mathrm{SSc}$ or SSc spectrum disease (including MCTD) even when the patients are symptom-free. ${ }^{1}$ In 


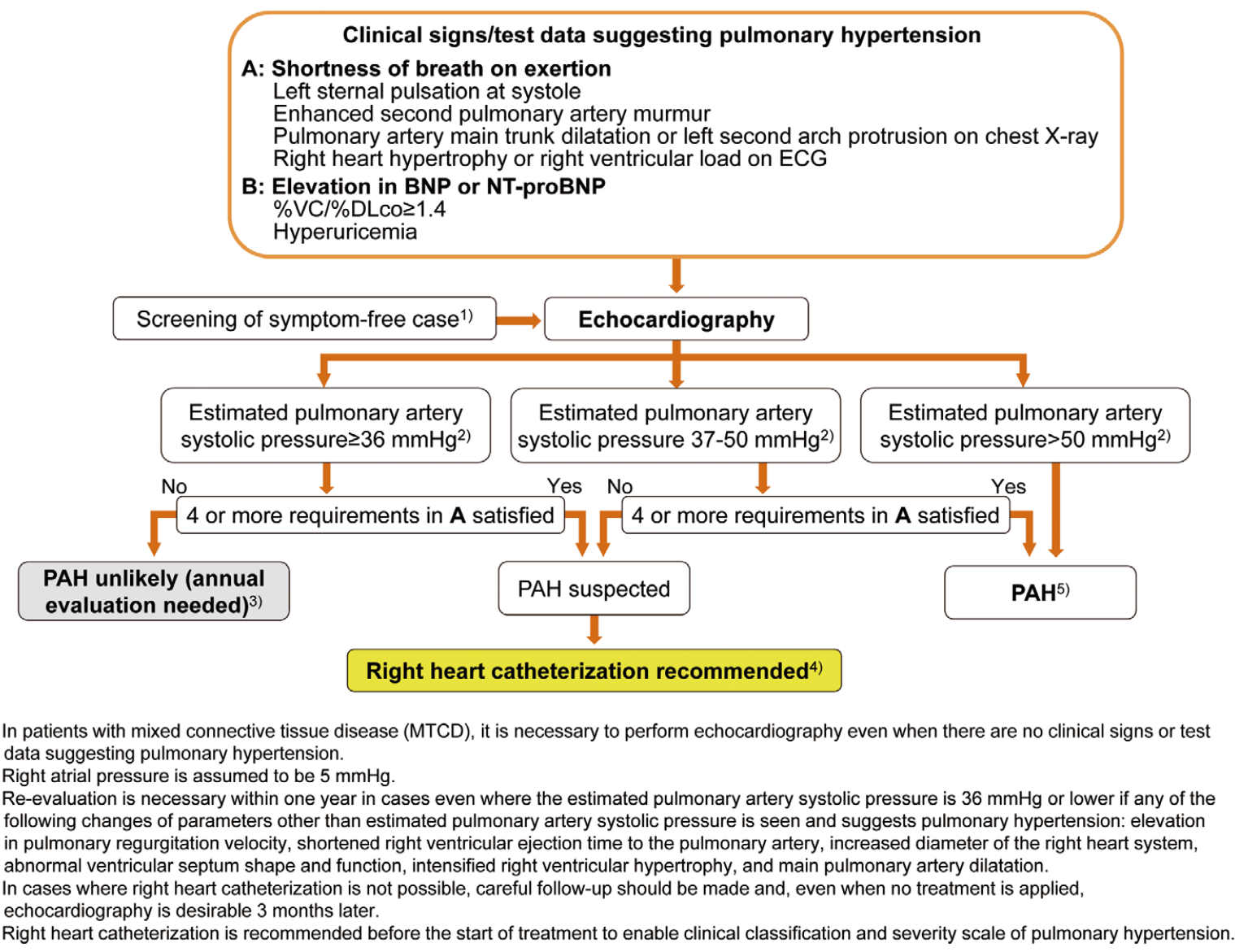

Figure 7. Guidelines to diagnosis of PAH in MCTD patients. (Note) Although right heart catheterization is not mandatory in this guide, it must be carried out before the start of pulmonary vasodilator therapy. (Source: Yoshida S, et al. 2011235)

cases in which the tricuspid regurgitation velocity (TRV) determined by Doppler ultrasound is $>3.4 \mathrm{~m} / \mathrm{sec}$ or the estimated right ventricular systolic pressure (eRVSP) calculated by adding RAP (estimated at $5 \mathrm{mmHg}$ ) to the tricuspid regurgitation pressure gradient (TRPG) is $>50 \mathrm{mmHg}$, the probability for presence of pulmonary hypertension is high and right heart catheterization for a definite diagnosis is recommended. ${ }^{1}$ However, even when TRV is $\leq 3.4 \mathrm{~m} / \mathrm{sec}$ or eRVSP is $\leq 50 \mathrm{mmHg}$, pulmonary hypertension may be present if the patient shows increases in the pulmonary valve regurgitation velocity, shortening of the acceleration time from the right ventricle to the pulmonary artery, dilatation of the right heart, including right atrium, flattening of the ventricular septum, right ventricular hypertrophy, or dilatation of the pulmonary artery main trunk. ${ }^{1}$ In a study of 137 patients with SSc designed to analyze the relationship of eRVSP (measured by Doppler ultrasound) to the mean pulmonary artery pressure (mPAP) determined by right heart catheterization, positive correlation was noted between these two parameters and there were about $10 \%$ false-negative cases (lack of TRPG elevation despite the presence of pulmonary hypertension) in addition to false-positive cases rated by Doppler ultrasound. ${ }^{229}$ Considering these cases, right heart catheterization should be performed in cases presenting with unexplained shortness of breath on exertion, physical signs suggesting pulmonary hypertension, or TTE-revealed abnormalities even when TRV is $\leq 3.4 \mathrm{~m} / \mathrm{sec}$ or eRVSP is $\leq 50 \mathrm{mmHg}$. The findings other than TTE findings shown to be useful in the screening of PAH include reduction in $\mathrm{DLCO}^{\mathbf{2 1 2 , 2 3 0 , 2 3 1}}$ as well as increases in blood BNP or NT-proBNP level and in serum uric acid level. ${ }^{232-234}$ Particularly in cases of SSc-PAH, reduction in DLco relative to forced vital capacity (FVC) is a characteristic sign (see 4.4 Echocardiography for Details Related to Echocardiography).

\section{d. Diagnosis of Pulmonary Hypertension}

For the diagnosis of pulmonary hypertension, measurement of pulmonary artery pressure (PAP) by right heart catheterization is indispensable, with the diagnosis of pulmonary hypertension made if mPAP at rest is $\geq 25 \mathrm{mmHg}$. Although right heart catheterization is not indispensable according to the "Guidelines to Diagnosis of Pulmonary Arterial Hypertension due to Mixed Connective Tissue Disease (MCTD)" in 2011 and the "Guidelines for diagnosis, severity classification, and treatment of systemic scleroderma" in 2016, both prepared in Japan, ${ }^{\mathbf{2 1 6}, 235}$ this test must be carried out before pulmonary vasodilators are prescribed.

Patients undergoing right heart catheterization should be selected by a combination of PAH risk factors and 


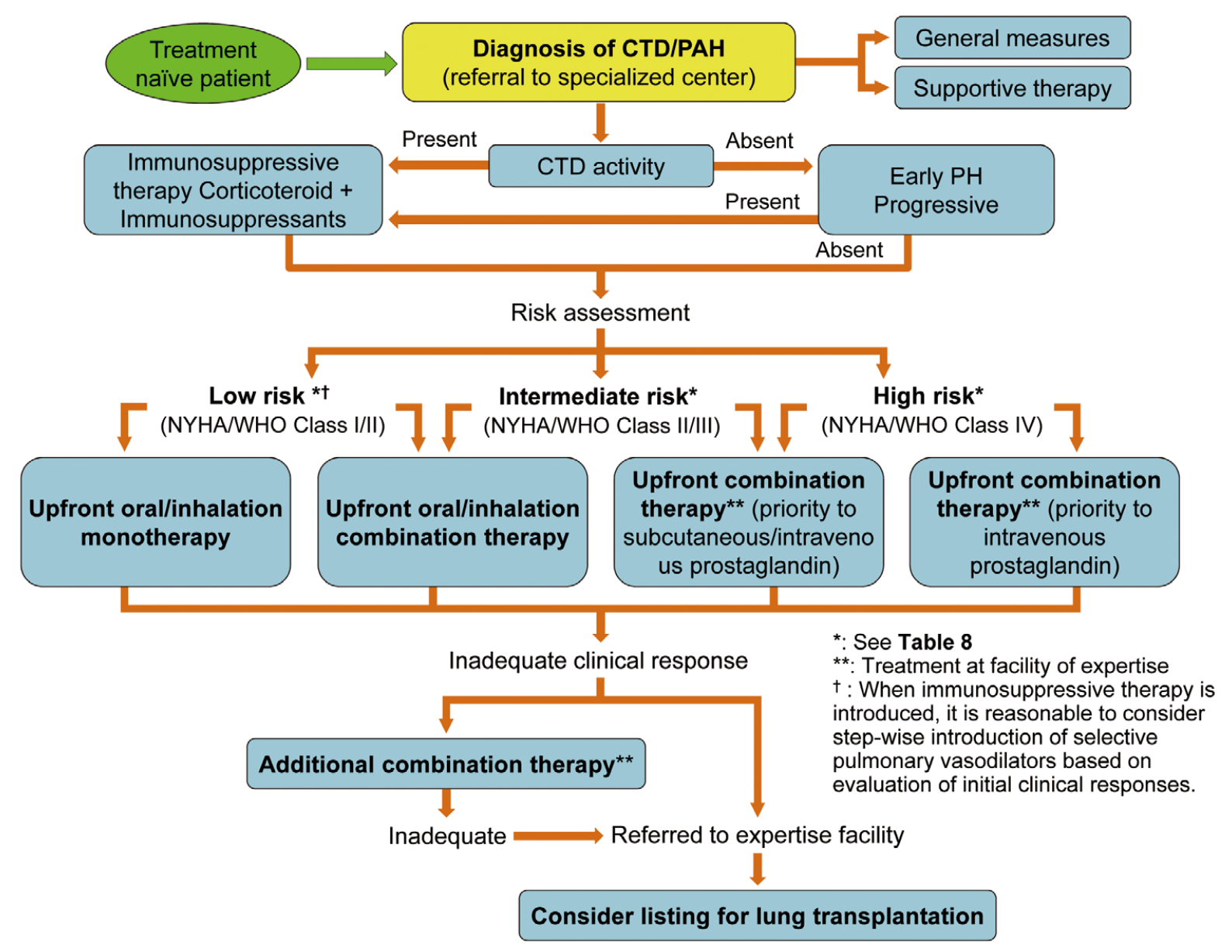

Figure 8. Treatment algorithm for CTD-PAH.

screening test results. In the multicenter study DETECT, carried out for the purpose of preparing criteria for optimal selection tool of patients for right heart catheterization, the eligibility criteria included disease duration of 3-year or longer and percentage of DLco relative to the predicted level (\%DLCO) $<60 \%$. ${ }^{226}$ During this study, the following parameters were selected from many noninvasive tests as useful means of SSc-PAH screening: increases in the ratio of $\% \mathrm{FVC}$ (percentage of FVC relative to predicted FVC) to $\% \mathrm{DLco}$, increases in serum NT-proBNP, and uric acid, and right axis deviation on ECG. Furthermore, taking into account also the TTE findings, the study proposed a twostage nomogram for selection of SSc cases requiring right heart catheterization (Figure 6). ${ }^{226}$ Screening using this nomogram has been shown to be more sensitive and less likely to overlook abnormalities compared to screening with TTE alone. In addition, the revised version of the "Guidelines to Diagnosis of Pulmonary Arterial Hypertension due to Mixed Connective Tissue Disease (MCTD)" prepared within the framework of Ministry of Health, Labour and Welfare Scientific Research Program in 2011 contains a diagnostic flow chart made of a combination of risk factors and screening test results (Figure 7). ${ }^{235}$ These guidelines, designed to include MCTD patients, assumes application also to patients with the other CTDs, although confirmation has not yet been completed.

\section{e. Clinical Classification of Pulmonary Hypertension}

Because patients with CTD can develop pulmonary hypertension of diverse clinical classifications, all patients diagnosed as having pulmonary hypertension require detailed pathophysiological evaluation. Patients satisfying all of the following requirements should be classified as having PAH: (1) PVR $\geq 3.0$ Wood Unit, (2) pulmonary artery wedge pressure (PAWP) $\leq 15 \mathrm{mmHg}$ (excluding pulmonary hypertension due to left heart disease), (3) absence of moderate or severer lung disease $(\% \mathrm{FVC} \geq 70 \%$ and percentage relative to predicted $\mathrm{FEV}_{1}\left[\% \mathrm{FEV}_{1}\right] \geq 60 \%$ ) (excluding pulmonary hypertension due to lung disease), and (4) lack of perfusion defect revealed by ventilation/perfusion lung scan consistent with a finding of pulmonary thromboembolism (excluding CTEPH). It has been shown that particularly in cases of SSc, the clinical classifications other than PAH cannot be completely ruled out by these criteria alone and it is not possible to conduct sufficient evaluation of mixed conditions ( $\mathrm{PAH}+$ other clinical classifications). ${ }^{\mathbf{2 1 0}}$

Evaluation of the lung histological findings from patients with SSc-PAH during lung transplantation or postmortem examination revealed a high frequency of pulmonary congestion at the capillary level compatible with the 
known features of PVOD. ${ }^{236}$ Furthermore, high-resolution CT (HRCT) of SSc-PAH patients frequently revealed PVOD-characteristic features, such as hilar/bronchial lymph node swelling, centrilobular ground glass opacity, and interlobular septum hypertrophy. The patients with these characteristics are often complicated by pulmonary edema following pulmonary vasodilator therapy, and their 3-year survival rate is poor (30\% or less). ${ }^{237}$ However, since PVOD-like HRCT features become apparent after long disease duration or after pulmonary vasodilator therapy it is difficult to detect occult PVOD at the early stages of the disease. Thus, some patients with SSc-PAH may have the complication of PVOD-like lesions, but detailed evaluation of such lesions at the time of diagnosis of PAH is difficult at present.

Even in symptom-free cases, patients with SSc are often found to have the complications of diastolic dysfunction of myocardium and myocardial fibrosis when examined by TTE or cardiac MRI. ${ }^{238-240}$ It is known that post-ischemic reperfusion injury due to vasoconstriction can induce minute necrosis, resulting in random distribution of small fibrotic foci across the myocardium..$^{241,242}$ It has been shown that pulmonary hypertension due to left heart disease with or without accompanying PAH is sometimes seen among the patients judged to be without pulmonary hypertension due to left heart disease in accordance with the criterion of PAWP (measured by right heart catheterization) $\leq 15 \mathrm{mmHg}$. Among patients with SSc, there are cases in which PAWP is discrepant from the left ventricular end-diastolic pressure (LVEDP) measured by left heart catheterization or LVEDP increases following fluid challenge with physiological saline even when the baseline LVEDP is $\leq 15 \mathrm{mmHg}$. In a study involving LVEDP measurement and evaluation following fluid challenge with physiological saline, features of pulmonary hypertension due to subclinical left heart disease were noted in 38\% of the cases ruled out as to pulmonary hypertension due to left heart disease and judged to have PAH on the basis of PAWP measured by right heart catheterization. ${ }^{243}$

\section{1.2.4 Treatment Algorithms}

\section{a. Treatment Outlined (Table 14)}

According to the ESC/ERS Guidelines for the Diagnosis and Treatment of Pulmonary Hypertension 2015, it is recommended to treat CTD-PAH in accordance with the IPAH/HPAH Treatment Guidelines. ${ }^{1}$ Algorithms of treatment are shown in the 2011 revised version of the "Guidelines to Diagnosis of MCTD Depending on Features in Individual Cases" ${ }^{244}$ and the "Systemic Scleroderma Diagnosis Criteria/Severity Classification/Clinical Management Guidelines" in $2016^{216}$ both prepared in Japan. This time, a new chart of therapeutic strategy for CTD-PAH was prepared in view of subsequent changes in therapeutic strategy and addition of new drugs available (Figure 8). Basic views and drugs used are identical to those for IPAH/ HPAH treatment, and their details are given in the Section on IPAH/HPAH. This section will focus on features specific to CTD-PAH. Calcium channel blockers recommended for treatment of IPAH/HPAH have been excluded from the chart because the percentage of long-term responders to calcium is less than $1 \%$ of all cases of CTD-PAH. ${ }^{1}$

\section{b. Immunosuppressive Therapy}

Immunosuppressive therapy is the mainstream for treatment of CTD in general. In addition to corticosteroids
(CS), CS pulse therapy and immunosuppressive agents are used depending on the severity of the disease. Since PAH is one of the organ manifestations of CTD, and it is highly likely that immunological mechanism is involved in its pathophysiology. In practice, effectiveness of immunosuppressive therapy for CTD-PAH has been shown in numerous reports, including case reports, case series, and retrospective studies. ${ }^{207,245-248}$ In these reports, the regimens of immunosuppressive therapy used and the treatment response criteria were inconsistent, and there was no randomized controlled trial. Therefore, the evidence for effectiveness of immunosuppressive therapy is insufficient. However, all patients who responded to the immunosuppressive therapy within 3 months of the treatment had favorable long-term prognosis and low relapse rate. ${ }^{245}$ Patients with SLE, MCTD or Sjōgren's syndrome often respond to immunosuppressive therapy. However, cases of SSc responding to immunosuppressive therapy are limited to case reports and there is no report of SSc cases judged as responders to immunosuppressive therapy in a retrospective study involving a larger number of patients. ${ }^{245,247}$ Therefore, immunosuppressive therapy is effective in a subset of patients with CTD-PAH, excluding cases of SSc. It has been reported that factors that predict a favorable response to immunosuppressive therapy include NYHA/WHO functional class I/II, preserved cardiac index, and early stage of PAH. ${ }^{246}$ To evaluate the treatment response, it is advised that right heart catheterization should be performed within a short period of time (within one month after the start of immunosuppressive therapy). In patients with NYHA/WHO functional class I or II, immunosuppressive treatment may be conducted without pulmonary vasodilators, but if clinical responses are insufficient at one month, introduction of pulmonary vasodilators should be initiated immediately.

There is no uniform regimen of immunosuppressive therapy. A treatment regimen employed in retrospective studies involving a relatively large number of subjects is a combination of moderate-to-high dose corticosteroid and cyclophosphamide (CYC). ${ }^{245-248}$ CYC was administered as an intermittent intravenous injection (a method extensively used for treatment of lupus nephritis), with the dosage at $500-1,000 \mathrm{mg} /$ dose or $600 \mathrm{mg} / \mathrm{m}^{2}$ (body surface area)/dose, and the frequency of dosing being 3-10 doses monthly. Because CYC involves risk for severe adverse reactions, such as malignancy and irreversible reproductive failure, further evaluation is needed about the dosage, dosing interval/period, and possibility of switching to other immunosuppressants.

\section{c. Pulmonary Vasodilators}

To date, no randomized placebo-controlled study has been conducted in patients with CTD-PAH or SSc-PAH. The only comparative study conducted to date was a study involving 111 patients with SSc-PAH allocating to the epoprostenol group and the conventional therapy group. ${ }^{249}$ At 12 weeks after the start of treatment, 6MWD and hemodynamics were significantly better in the epoprostenol group than in the conventional therapy group. In a retrospective study in patients with CTD-PAH, the survival was improved significantly in the epoprostenol group compared to the historical control group with identical background variables. ${ }^{250}$ Later, in randomized placebo-controlled studies in patients with $\mathrm{PAH}$, including about $20-30 \%$ of patients with CTD-PAH, sub-analysis was conducted on the CTDPAH cases. When sub-analysis of the data from the CTD- 


\begin{tabular}{|l|c|c|c|c|}
\hline Table 15. PoPH Severity Rating and Treatment & Normal & Mild & Moderate & Severe \\
\hline NYHA functional class & & I, II & II, III & III, IV \\
\hline Mean pulmonary artery pressure $(\mathrm{mmHg})$ & $15-24$ & $25-34$ & $35-44$ & $>45$ \\
\hline Cardiac index $\left(\mathrm{L} / \mathrm{min} / \mathrm{m}^{2}\right)$ & $2.5-4.0$ & $>2.5$ & $>2.5$ & $<2.0$ \\
\hline Pulmonary vascular resistance $\left(\right.$ dyne $\left.\cdot \mathrm{sec} \cdot \mathrm{cm}^{-5}\right)$ & $<240$ & $240-500$ & $500-800$ & $>800$ \\
\hline Right atrial pressure $(\mathrm{mmHg})$ & $0-5$ & $0-5$ & $5-8$ & $>8$ \\
\hline Prognosis & & Good & Unknown \\
\hline Pulmonary vasodilator therapy & & Unknown & Considered & Noor \\
\hline Improvement after liver transplantation & & Good & Unknown \\
\hline
\end{tabular}

(Use of pulmonary vasodilators for treatment of $\mathrm{PoPH}$ in a manner similar to the recommendation based on IPAH/HPAH severity level: Recommendation Class I, Evidence Level C) (Source: Prepared based on Hoeper MM, et al. 2004269)

PAH patients was carried out by setting the 6MWD as a primary endpoint, there was no significant difference in 6MWD between the drug treatment group (treated with bosentan or riociguat) and the placebo group..$^{251,252}$ In the sub-analysis of the data from CTD-PAH patients treated with sildenafil, the result as to the primary endpoint differed significantly between the three times daily sildenafil $20 \mathrm{mg}$ treatment group and the placebo group, but the difference was not dose-dependent and was not significant at higher doses. ${ }^{253}$ In sub-analysis of the data from a placebo-controlled study of CTD-PAH patients with the morbidity and mortality event as a primary endpoint, there was no significant difference in the event between the macitentan treatment group and the placebo group, ${ }^{\mathbf{1 3 8}}$ whereas a significant difference was noted between the selexipag group and the placebo group. ${ }^{137}$ However, when these results are interpreted, it needs to be considered that comparison among these studies is difficult because of difference in eligibility criteria and background variables of patients among individual studies. For example, in the above-mentioned study using sildenafil, the percentage of poor prognosis SSc-PAH cases was $45 \%$, markedly lower than the percentage in the other studies ( $60 \%$ or more). ${ }^{253}$ In the study on selexipag, the number of CTD-PAH patients enrolled was 334 cases, larger than that in any other past study, and patients with a predominant $\mathrm{PAH}$ feature (PVR > 5.0 Wood Unit) were selectively enrolled. ${ }^{137}$ In a randomized study comparing the initial combination therapy (ambrisentan plus tadalafil treatment) with monotherapy using each drug, the primary endpoint (morbidity and mortality event) was reduced significantly in the initial combination therapy group compared to the monotherapy group. ${ }^{\mathbf{1 4 4}}$ The significant difference was reproduced also in sub-analysis of the data limited to patients with CTD-PAH or SSc-PAH, ${ }^{254}$ but this study excluded patients with pulmonary hypertension due to left heart disease. ${ }^{144}$

On the basis of these results, it is that pulmonary vasodilators are effective against CTD-PAH but that the response to this therapy varies depending on not only the underlying disease but also on the extent of combined other clinical classifications of pulmonary hypertension. As in patients with IPAH/HPAH, patients with CTD-PAH often respond to initial combination therapy, but there are also some cases of CTD-PAH showing aggravation of pulmonary congestion or hypoxemia following addition or dose escalation of pulmonary vasodilators. ${ }^{255}$ Accumulating data indicate that the long-term survival is favorable in cases in which pulmonary vasodilator therapy is started early and can be continued without any problem with tolerability. ${ }^{\mathbf{2 5 6}, 257}$

\section{d. Conclusions}

In patients with CTD, the risk of pulmonary hypertension is high and early detection is necessary by a screening program corresponding to the risk factors of individual patients. Patients with CTD can have disease of diverse clinical classifications, including PAH, PVOD, pulmonary hypertension due to left heart disease, pulmonary hypertension due to lung disease (e.g., interstitial lung disease), CTEPH, and pulmonary hypertension due to pulmonary arteritis. In addition, patients with SSc frequently have a condition consisting of a mixture of several clinical classifications of pulmonary hypertension. Treatment of CTDPAH patients is performed using the guidelines for IPAH/ HPAH treatment. Immunosuppressive therapy is sometimes effective in cases of PAH associated with SLE, MCTD, or primary Sjōgren's syndrome. Careful treatment with pulmonary vasodilators is needed in cases of a mixture of several classifications of pulmonary hypertension due to SSc.

\subsection{Pulmonary Arterial Hypertension Associated With Portal Hypertension (PoPH)}

\section{Summary}

The liver cross-talks with various organs. There are two characteristic disease groups classified on the basis of association with liver disease and pulmonary circulation: (1) hepatopulmonary syndrome (HPS), which involves reduction in PVR due to marked pulmonary artery dilatation and presents with severe hypoxia as a major sign, and (2) portopulmonary hypertension (PoPH), which involves increases in PVR and presents with pulmonary hypertension. ${ }^{17,258,259} \mathrm{PoPH}$ is a type of PAH that develops due to portal hypertension regardless of the severity level of liver disease. ${ }^{\mathbf{6}, 260}$ There are rare cases in which these two conditions overlap, e.g., cases of PoPH arising from HPS. ${ }^{261}$ The pathophysiology of $\mathrm{PoPH}$ has not been fully clarified although genetic predisposition to this condition has also been reported. The pathological state of the pulmonary artery in patients with advanced PoPH resembles that of other types of PAH including idiopathic PAH. Typically, high cardiac output is seen at the early stages of this disease, followed gradually by reduction in cardiac function and an increase in PVR. The prognosis is poor unless treated. Treatment of PoPH is basically performed in a manner similar to that for PAH. Regarding use of the drugs for treatment of pulmonary hypertension, there are several reports of effectiveness in a small number of cases. However, in conventional randomized controlled trials of drugs specific to treatment of $\mathrm{PAH}$, cases of $\mathrm{PoPH}$ are often 
excluded completely or only a small number of PoPH cases are included. For this reason, adequate data on efficacy or safety of such drugs in the treatment of PoPH cannot be collected in such studies, thus requiring careful judgment about selection of drugs for treatment and their dosing methods. Liver transplantation is considered in cases indicated for liver transplantation, but the prognosis after liver transplantation is poor in patients with severe pulmonary hypertension. If mPAP is improved by the drugs for treatment of pulmonary hypertension, the safety of liver transplantation may be improved. The severity rating and treatment of PoPH are shown in Table 15.

\section{1.3.1 Epidemiology/Etiology}

As its name suggests, $\mathrm{PoPH}$ is a type of $\mathrm{PAH}$ associated with portal hypertension. The presence of portal hypertension is related to the features of this disease but hepatic dysfunction is not always seen. However, cases complicated by liver cirrhosis account for the highest percentage of PoPH patients. ${ }^{260}$ According to a study in France, PoPH is the fourth most frequent type of PAH next to IPAH, CTD-PAH, and CHD-PAH, ${ }^{11}$ and has been reported to be seen in 2-6\% of all patients with portal hypertension. . $^{16,260,262}$ Because of its onset mechanism, PoPH was classified as Group 1 (PAH) in the Nice Classification (2013). Regarding the etiology of $\mathrm{PoPH}$, high cardiac output increases from the appearance of a shunt and dilatation of systemic vessels, and that PVR is initially normal. Later, the shear stress to the pulmonary vessels increases, followed by pulmonary artery tunica intima hypertrophy and pulmonary artery remodeling, possibly leading to increases in PVR by obstruction/stenosis of the pulmonary artery. Histological features of $\mathrm{PoPH}$ include hypertrophy of vascular tunica intima and smooth muscle of vascular tunica media, plexiform lesions in vascular lumen, necrotic vasculitis/fibrinoid necrosis, and microthrombus. There is also a view that the formation of shunt due to liver disease and the reduction in hepatic metabolism lead to inflow of metabolites (serotonin) from enterohepatic circulation to pulmonary circulation, possibly leading to pulmonary hypertension by constriction of pulmonary vessels. ${ }^{259}$ Regarding the involvement of genetic factors in the onset of $\mathrm{PoPH}$, single nucleotide polymorphism (SNP) of estrogen receptor 1, calcium-bound protein A4, aromatase, PDE5, and angiopoietin 1 genes has been reported, suggesting the involvement of genetic mechanism in estrogen signal and cell proliferation regulation. ${ }^{263}$

\section{1.3.2 Diagnosis}

The symptoms of PoPH are basically similar to those of the other types of PAH. However, in cases accompanied by symptoms of liver disease, the diagnosis of PAH may be difficult. If symptoms develop in patients with liver disease or portal hypertension and patients indicated for liver transplantation, it is recommended to conduct screening of pulmonary hypertension by echocardiography.,16 The diagnosis of $\mathrm{PoPH}$ is conducted by processes similar to those for diagnosis of PAH associated with other underlying disease. A precaution needed is that the coexistence of portal hypertension and pulmonary hypertension does not immediately lead to the diagnosis of PoPH. Sufficient distinction from other related diseases is essential. Right heart catheterization is beneficial because it allows simple estimation of portal pressure by measurement of portal vein wedge pressure in addition to its role indispensable in diagnosis and severity rating. Furthermore, some other tests including diagnostic imaging may allow estimation of portal hypertension. In general, patients with PoPH (at least early-stage cases) tend to have high cardiac output and low PVR compared to patients with IPAH. ${ }^{264}$

\section{1.3.3 Prognosis and Severity Rating}

At early stages of $\mathrm{PoPH}$, reduction in systemic vascular resistance and the resultant increase in cardiac output may be seen. In such cases, PAP increases by increased cardiac output, leading to reduction in PVR. Although progression of the disease in some cases is not as rapid as that of the other types of PAH, the function of the right heart system decreases with time, leading to increases in PVR and aggravation of the condition. PoPH has poor prognosis. According to data from the Mayo Clinic, the five-year survival rate of patients with PoPH was $14 \%$ when left untreated, with $54 \%$ of patients dying within 1 year after diagnosis. ${ }^{265}$ If only PoPH treatment was performed without liver transplantation, the five-year survival rate was $45 \%$, and the five-year survival rate was improved to $67 \%$ in cases in which liver transplantation was possible to perform. ${ }^{266}$ Right heart catheterization is very important in rating the severity of $\mathrm{PoPH},{ }^{267}$ and treatment should be considered depending on the severity level ${ }^{268,269}$ (Table 15).

\section{1.3.4 Treatment}

As a rule, PoPH is treated by the methods usually performed in cases of $\mathrm{PAH}$, thereby taking into consideration the severity level of the underlying liver disease. ${ }^{1}$ In cases of mild PoPH without symptoms or signs of vascular lesions and presenting with high cardiac output as a major symptom (particularly in patients with PVR $<3.0$ Wood Unit), no treatment is usually needed, and there is no definite evidence for the significance of early intervention with drugs for treatment of pulmonary hypertension. However, in view of the nature of PAH, periodical observation is necessary even in cases in which the disease is mild and therapeutic intervention is judged as unnecessary.

Diuretics need to be used carefully. Blood flow through vessels increases in the presence of right heart failure or chronic liver disease, and diuretics exert efficacy by reducing blood flow. However, this effect can reduce pre-loads and hence reduce cardiac output. Anticoagulant therapy is effective against PAH or CTEPH, but it is usually not recommended for patients with liver cirrhosis because they often develop complication by thrombocytopenia (associated with reduced hepatic synthesis clotting factors and splenoma) and gastrointestinal varices that can be fatal if bleeding occurs. ${ }^{270}$ It is also not recommended to use $\beta$-blockers that reduce portal pressure, on the grounds that hemodynamics and exercise tolerance can be aggravated by them in patients with PoPH. ${ }^{271}$

Evidence for intervention with pulmonary vasodilators at present primarily pertains to IPAH, and patients with $\mathrm{PoPH}$ are often excluded from randomized controlled trials. As a result, there is little evidence as to whether the efficacy and safety of pulmonary vasodilators are seen also in patients with PoPH. Thus, caution is needed when making a judgment. However, in small-scale studies, effectiveness of pulmonary vasodilators in patients with $\mathrm{PoPH}$ were demonstrated. ${ }^{272-275}$ Regarding the endothelin pathway, there is a report that increases in blood endothelin-1 level was noted in patients with $\mathrm{PoPH},{ }^{276}$ suggesting the effectiveness of ERA from the aspect of mechanisms. There is 


\begin{tabular}{|c|c|c|c|c|c|c|}
\hline \multicolumn{3}{|c|}{ Clinical classification } & \multicolumn{2}{|c|}{ Recommendation } & $\begin{array}{l}\text { Recommendation } \\
\text { Class }\end{array}$ & $\begin{array}{l}\text { Evidence } \\
\text { Level }\end{array}$ \\
\hline \multicolumn{3}{|c|}{$\begin{array}{l}\text { 1. Residual PAH after shunt repair (biventricular } \\
\text { repair): PAH remaining and/or exacerbating soon } \\
\text { after surgery/PAH occurring (onset) much time after } \\
\text { operation }\end{array}$} & \multicolumn{2}{|l|}{ Similar to IPAH/HPAH } & - & - \\
\hline \multirow{20}{*}{$\begin{array}{l}\text { 2. Unrepaired } \\
\text { shunt }\end{array}$} & \multirow{6}{*}{$\begin{array}{l}\text { 2.1. Non- } \\
\text { Eisenmenger's } \\
\text { syndrome }\end{array}$} & \multirow{2}{*}{$\begin{array}{l}\text { 2.1.1. (I) PAH } \\
\text { accompanied by } \\
\text { small (restrictive) } \\
\text { shunt hole }\end{array}$} & \multicolumn{2}{|c|}{$\begin{array}{l}\text { Basically similar to IPAH/HPAH, except for } \\
\text { the shunt closing procedure }\end{array}$} & - & - \\
\hline & & & \multicolumn{2}{|l|}{ Shunt closure } & III & C \\
\hline & & & \multicolumn{2}{|c|}{$\begin{array}{l}\text { Basically similar to Eisenmenger's syndrome, } \\
\text { except for the shunt closing procedure }\end{array}$} & - & - \\
\hline & & 2.1.2. $\mathrm{PAH}$ due & \multicolumn{2}{|c|}{$\begin{array}{l}\text { Shunt closure in patients with PVR }(I)<2.3 \\
\text { Wood Unit }\left(4 \text { Wood Unit } \cdot \mathrm{m}^{2}\right)\end{array}$} & Ila & $\mathrm{C}$ \\
\hline & & $\begin{array}{l}\text { to large (non- } \\
\text { restrictive) shunt }\end{array}$ & \multicolumn{2}{|c|}{$\begin{array}{l}\text { Shunt closure in patients with } 2.3 \text { Wood Unit } \\
\left(4 \text { Wood Unit } \cdot \mathrm{m}^{2}\right) \leq \text { PVR }(\mathrm{I}) \leq 4.6 \text { (8 Wood } \\
\left.\text { Unit } \cdot \mathrm{m}^{2}\right)\end{array}$} & Illb & C \\
\hline & & & \multicolumn{2}{|c|}{$\begin{array}{l}\text { Shunt closure in patients with PVR }(\mathrm{I})>4.6 \\
\text { Wood Unit }\left(8 \text { Wood Unit } \cdot \mathrm{m}^{2}\right)\end{array}$} & III & C \\
\hline & \multirow{14}{*}{\multicolumn{2}{|c|}{ 2.2. Eisenmenger's syndrome }} & \multirow{3}{*}{$\begin{array}{l}\text { Use of pulmonary } \\
\text { vasodilators in cases } \\
\text { of NYHA/WHO } \\
\text { functional class I/II }\end{array}$} & Oral & Ila & B \\
\hline & & & & Inhalation & $\mathrm{Ilb}$ & C \\
\hline & & & & $\begin{array}{l}\text { Subcutaneous/ } \\
\text { intravenous }\end{array}$ & $\mathrm{Ilb}$ & C \\
\hline & & & $\begin{array}{l}\text { Upfront use of } \\
\text { pulmonary vasodila- } \\
\text { tors in cases of } \\
\text { NYHA/WHO func- } \\
\text { tional class I/II }\end{array}$ & All & Ilb & C \\
\hline & & & \multirow{4}{*}{$\begin{array}{l}\text { Use of pulmonary } \\
\text { vasodilators in cases } \\
\text { of NYHA/WHO func- } \\
\text { tional class II/IV }\end{array}$} & Bosentan & 1 & B \\
\hline & & & & $\begin{array}{l}\text { Oral drugs other } \\
\text { than bosentan }\end{array}$ & Ila & B \\
\hline & & & & Inhalational drugs & Ila & B \\
\hline & & & & $\begin{array}{l}\text { Drugs for subcutane- } \\
\text { ous/intravenous } \\
\text { injection }\end{array}$ & $\mathrm{Ilb}$ & C \\
\hline & & & $\begin{array}{l}\text { Upfront use of } \\
\text { pulmonary vasodila- } \\
\text { tors in cases of } \\
\text { NYHA/WHO func- } \\
\text { tional class III/IV }\end{array}$ & All & $\mathrm{Ilb}$ & C \\
\hline & & & \multirow{2}{*}{$\begin{array}{l}\text { Use of two or more } \\
\text { vasodilators in all } \\
\text { cases poorly } \\
\text { responding to single } \\
\text { oral drug }\end{array}$} & $\begin{array}{l}\text { Oral/inhalational } \\
\text { drugs }\end{array}$ & Ila & B \\
\hline & & & & $\begin{array}{l}\text { Drugs for subcutane- } \\
\text { ous/intravenous } \\
\text { injection }\end{array}$ & $\mathrm{Ilb}$ & C \\
\hline & & & \multicolumn{2}{|c|}{ Shunt closure } & III & C \\
\hline & & & \multicolumn{2}{|c|}{$\begin{array}{l}\text { (Cardio)pulmonary transplantation (+intracar- } \\
\text { diac repair) for cases of medication-refractory } \\
\text { progressive Eisenmenger's syndrome) }\end{array}$} & Ila & B \\
\hline & & & \multicolumn{2}{|c|}{ Contraception } & I & C \\
\hline
\end{tabular}

a study, although conducted on a small scale, demonstrating that treatment with bosentan (an ERA) extended the survival period of $\mathrm{PoPH}$ patients and reduced recurrence of right-sided heart failure, ${ }^{272}$ and there is also a study report on improved hemodynamics after ambrisentan treatment. ${ }^{277}$ Because bosentan cannot be recommended due to hepatopathy as an adverse reaction, clinical introduction of macitentan has expanded the alternatives for treatment together with ambrisentan. In any event, periodical followups are needed concerning the hepatic function of patients. PDE5 inhibitors are also effective and can reduce PVR. Treatment with sildenafil has been reported to have resulted in improvement of 6MWD one year later. ${ }^{274}$ In recent years, upfront combination therapy, which involves combined drug treatment at the early stages, has begun to be used occasionally, but there is little data available in cases of $\mathrm{PoPH}$, thus requiring caution in adoption of this therapy.

Liver transplantation is a method of treatment specific to PoPH. Liver transplantation is not recommended for the purpose of treating pulmonary hypertension. It is considered in indicated cases judged on the basis of the liver disease severity level. In cases of severe pulmonary hypertension, liver transplantation is not recommended because of poor perioperative prognosis. According to data from the Mayo Clinic, the prognosis after liver transplan- 
tation is relatively favorable in patients with mPAP not exceeding $35 \mathrm{mmHg} .{ }^{278} \mathrm{In}$ cases of $\mathrm{PoPH}$ judged as requiring liver transplantation, improving the hemodynamics by pulmonary vasodilators should to be attempted before transplantation, and the indications for transplantation are supported by the finding of improvement in response to such treatment. ${ }^{\mathbf{2 6 6}, 279,280}$ The prognosis after transplantation is poor in cases in which the pre-transplantation $\mathrm{mPAP}$ is $\geq 35 \mathrm{mmHg}$. For this reason, the goal of pulmonary hypertension treatment is set at $\mathrm{mPAP}<35 \mathrm{mmHg}$ and PVR $<250$ dyne $\cdot \mathrm{sec} \cdot \mathrm{cm}^{-5} \cdot{ }^{\mathbf{2 7 8 , 2 8 1}}$ In any event, any surgery under general anesthesia, including transplantation, involves risk in patients with pulmonary hypertension, and the indications for surgery needs to be carefully judged.

\section{I1.4 Pulmonary Arterial Hypertension Associated With Adult Congenital Heart Disease}

\section{Summary}

Pulmonary hypertension arising from shunt-related disorders in congenital heart disease (CHD) is classified as Group 1 (PAH) according to the Nice Classification (2013). This disease is etiologically considered as obstructive pulmonary artery disease arising from pulmonary artery endothelial disorder due to an early-stage massive left-toright shunt related increase in pulmonary blood flow. The degree of histological disorders involved is expressed in Heath-Edwards Classification, with Grade 4 or severer lesions considered as irreversible. The speed of disorder progression depends on the size and location of the shunt. What is particularly important is whether the shunt is located on the venous side of the pulmonary atrioventricular valve (tricuspid valve in normal concordance) or on the arterial side of the same valve. A large hole in the latter case (e.g., VSD) is known to advance to uncorrectable irreversible lesion (Eisenmenger's syndrome) if not repaired by 1-2 years after birth. In the former case (e.g., atrial septum defect [ASD]), pulmonary hypertension is often absent and, even when it is present, its symptoms are mild and often overlooked until adulthood. In any event, if pulmonary hypertension is left untreated after onset, the obstructive pulmonary artery lesion increases sooner or later, leading to a considerable increase in the right-to-left shunt volume, resulting in cyanosis. Such a condition is classified as Eisenmenger's syndrome and considered as the irreversible/progressive terminal stage. This section deals only with PAH associated with adult CHD (ACHD), i.e., ACHD-PAH. See Chapter II "6. Pulmonary Hypertension in Children" for pediatric CHD-PAH.

The most important feature of CHD-PAH is that the histological findings and response to drugs for treatment of pulmonary hypertension are quite similar to those of IPAH. This similarity is very useful in considering treatment of this disease. However, to be accurate, it is difficult to propose uniform treatment guidelines to treat individual cases of CHD-PAH if we consider inter-individual variances such as presence/absence of a residual shunt, diversity of the underlying congenital cardiovascular anomaly, modification by the history of repair and palliative operation, complication by systemic/psychiatric disorders due to chromosomal aberration, complication of organ failure other than cardiovascular disorders, and problems related to pregnancy and delivery. Despite such circumstances, it is useful for this disease group to assess the pathophysiology and consider treatment by subdividing ACHD-PAH into the four groups as shown in Table 16. In these guidelines, diagnosis/treatment will be described in accordance with this classification.

\section{1.4.1 Epidemiology}

The prevalence of CHD among newborns has been reported to be about $1 \%$ regardless of race. Following the establishment of open heart surgery with the well-developed artificial heart-lung systems in the 1970 s and advances in rapid management after birth of such newborns (including advances in pre-birth diagnosis), more than $90 \%$ of patients with CHD can reach adulthood. Following the recent advances in healthcare and its system, the percent increase in the number of patients with CHD-PAH will over time continue to decrease, accompanied by a lower increase (increase rate) in the number of patients with Eisenmenger's syndrome. However, regarding CHD-PAH which is caused by ASD on the venous side of the pulmonary atrioventricular valve (tricuspid valve in normal cases), symptoms are difficult to identify both subjectively and objectively and the number of patients developing this disease will change little over time. At present, the number of patients with CHD and the percentage of CHD-PAH patients among such patients remain unknown. As of 2007, the number of patients with ACHD in Japan was estimated to be more than $400,000 .{ }^{282}$ According to more recent surveys, the percentage of patients with CHD-PAH among all patients with CHD was 3-10\%. ${ }^{283,284}$ Based on these findings, the number of patients with ACHD is estimated to be about 12,000-40,000 in Japan. Thus, the percentage of ACHD-PAH patients among PAH patients is quite high, suggesting that this is a major group of $\mathrm{PAH}$.

\section{1.4.2. Diagnosis}

Regarding the diagnosis and treatment of ACHD-PAH patients, it is very important clinically to assess which type of the disease shown in Table 16 is applicable to individual cases. Important symptoms of ACHD, regardless of presence/absence of repair, are shortness of breath, cyanosis, and clubbed finger. Determining complication by pulmonary hypertension based on the presence/absence of these symptoms is important in the differential diagnosis of patients presenting with these symptoms. In ACHD patients, ECG, chest X-ray, and TTE should be conducted as routine tests. If TTE reveals increases in the pressure gradient across the pulmonary atrioventricular valve (tricuspid valve in normal cases) (TRPG), the general steps for diagnosis of pulmonary hypertension should be taken in accordance with the algorithm shown in Figure 1, thereby distinguishing the condition from shunt-related ACHD-PAH. Other important points requiring attention during the tests at this stage are shown below.

- ECG: Simple anomalies, such as ASD and VSD, usually present with right-sided heart loads, whereas in complex heart malformation and dextrocardia/situs inversus, it is difficult to interpret ECG.

- Chest X-ray: Marked pulmonary artery protrusion/ pulmonary aneurysm often suggest pulmonary high flow and PAP enhancement, but some other factors, such as pulmonary artery (valve) stenosis, may be responsible.

- Echocardiography: This is the most reliable tool of screening for diagnosis of pulmonary hypertension. If increased TRPG is noted, echocardiography should be performed with close attention to the presence/absence of stenosis in the pulmonary ventricle (right ventricle in 
normal cases) outflow tract and the route of pulmonary artery, and if this test reveals that stenosis is absent or mild, the patient should be strongly suspected of having complication by pulmonary hypertension. Patients with Eisenmenger's syndrome show wall thickness increase in a similar degree in the lung (right) ventricle and the systemic (left) ventricle. Right atrium dilatation is seen in cases of advanced IPAH. In cases complicated by ASD (involving shunt on the venous side of pulmonary atrioventricular valve) or partial anomaly of pulmonary vein return (PAPVR), right atrium dilatation is often seen even in the absence of pulmonary hypertension/ $\mathrm{PAH}$, depending on the left-to-right shunt volume. Conversely, detailed examination following finding of this abnormality occasionally leads to the detection of ASD/PAPVR and/or PAH.

- Transesophageal echocardiography (TEE): This is indispensable for determining the indications of ASD patients for percutaneous closure operation. Ruling out, evaluation, and other steps are taken concerning intracardiac shunt (PAPVR) as needed.

- Coronary CT angiography: This test should be performed in advance for all patients that will undergo cardiac catheterization unless it is contraindicated. ECG-gated slice imaging allows accurate structural evaluation, including the heart and lungs. In addition to evaluation of the target shunt and various structural anomalies/ shunts/collaterals, this test allows avoidance of overlooking other accompanying malformations. CT scan is basically needed for the ruling out/evaluation of the possibility as to the presence/absence of PAPVR, VSD (small defects often developing in muscles), patent ductus arteriosus (PDA), various collaterals (major aorto-pulmonary collateral arteries (MAPCA), and various thrombi/emboli. Also for evaluation of ASD, coronary artery CT before TEE is important for smooth evaluation. In addition, it allows evaluation of other accompanying malformations as well as stenosis, dilatation, and other anomalies of the pulmonary artery, and aorta. In the end, legs also need to be scanned to ensure a route for accessing the heart with the catheter.

- cardiac MRI (cMRI): The cardiac function of ACHD patients reflects the influence from a complex mixture of various forms of ventricle, various contracting styles (of a wide range of QRS), abnormal atrium-ventricle-large vessel linkage, and postoperative factors. Therefore, cMRI for three-dimensional analysis and accurate evaluation of vascular blood flow is quite useful. This test allows accurate assessment of the pulmonary/systemic blood flow ratio (Qp/Qs) (flow analysis of the arteries and large veins, analysis of ventricular capacity, and valvular regurgitation volume) and accurate evaluation of each ventricle function. This test does not always need contrast material and is hence less invasive, but has shortcomings of taking much time and inability to yield satisfactory images in mentally retarded patients.

- Cardiac catheterization: This is indispensable for a definite diagnosis of PAH/pulmonary hypertension and evaluation of cardiac function. Although accurate evaluation with this test is occasionally difficult depending on the location of shunt and other factors, this test provides valuable information concerning PAP, PVR, and Qp/Qs. If a definite diagnosis of pulmonary hypertension is made, followed by differential diagnosis of the group of pulmonary hypertension, the diagnosis of ACHD-PAH can be established. However, it is sometimes difficult to distinguish PAH from Group 5 segmental pulmonary hypertension.

- Ventilation/perfusion lung scan: This test is usually needed for evaluation of pulmonary embolism-related disease such as CTEPH and differential diagnosis of the group of pulmonary hypertension. In the management of ACHD, it is also used for evaluation of perfusion ratio between right and left lungs and right-to-left shunt rate. When the site of radioisotope injection is decided, care needs to be taken of the presence of malformation, such as residual left superior vena cava, to avoid erroneous evaluation of the shunt rate. It is therefore necessary to perform a ventilation/perfusion lung scan appropriately after diagnostic imaging (including CT scan mentioned above).

If a diagnosis of ACHD-PAH has been made on the basis of the test results mentioned above, then the condition is classified into one of the 4 groups shown in Table 16, and a treatment plan is devised. In cases in which the history of pulmonary hyperperfusion has been demonstrated, a diagnosis of shunt-related PAH (other than 2.1.1, Table 16) is made. In patients without the history of pulmonary hyperperfusion and having small shunt not causing pulmonary hyperperfusion, a diagnosis of (I) PAH accompanied incidentally by small shunt $(2.1 .1$, Table 16) is made.

\section{1.4.3 Severity Rating}

Regarding ACHD-PAH after shunt repair and (I) PAH accompanied by small shunt, the severity is rated with reference to the criteria for IPAH. The severity of ACHDPAH without shunt repair varies depending on whether the case satisfies the criteria for diagnosis of Eisenmenger's syndrome, but its judgment is not easy. Even in cases diagnosed as having Eisenmenger's syndrome absent, the prognosis has not always been favorable after clinical introduction of oral-dose drugs for treatment of pulmonary hypertension. ${ }^{285}$ The severity rating after clinical introduction of the drugs for treatment of pulmonary hypertension will be established on the basis of evidence for treatment, but CHD-PAH with lower response to medication may be higher in severity.

\section{1.4.4 Guidelines to Treatment}

Table 16 shows the management and treatment recommended for Eisenmenger's syndrome. Regarding Eisenmenger's syndrome, the efficacy of drugs used for treatment of PAH and their effect in improving the prognosis have been shown in a randomized placebo-controlled study, ${ }^{286}$ a large-scale registry study ${ }^{\mathbf{2 8 7}}$ and (retrospective) observational studies. ${ }^{288-290}$ Depending on the evidence level for each drug, in cases of NYHA/WHO functional class III/IV, bosentan is a drug with Class I indications (Evidence Level B) and the other oral-dose, inhalational, subcutaneous, and intravenous drugs for treatment of PAH are drugs of Class IIa indications (oral-dose and inhalational: Evidence Level B, subcutaneous and intravenous: Evidence Level C). Also in cases of NYHA/WHO Functional Class I/II, the efficacy of drugs used for PAH treatment has been reported in a registry study, ${ }^{291}$ and all oral-dose drugs for PAH treatment are recommended as Class IIa (Evidence Level B). Upfront combination therapy and treatment with inhalational/intravenous/subcutaneous drugs are Class IIb indications (Evidence Level C). In cases in which the response to uncombined oral drug therapy is insufficient, 


\begin{tabular}{|l|l|}
\hline \multicolumn{2}{|l|}{ Table 17. Classification of Drug- and Toxin-Induced PAH (Nice Conference in 2013) } \\
\hline Definite & Possible \\
\hline Aminorex & Cocaine \\
Fenfluramine & Phenylpropanolamine \\
Dexfenfluramine & St. John's wort \\
Toxic rapeseed oil & Chemotherapeutic agents (mitomycin C, cyclophosphamide) \\
Benfluorex & Interferon $a$ and $\beta$ \\
Selective serotonin reuptake inhibitors & Amphetamine-like drugs \\
\hline Likely & Unlikely \\
\hline Amphetamines & Oral contraceptives \\
L-tryptophan & Estrogen \\
Methamphetamine & Cigarette smoking \\
Dasatinib & \\
\hline
\end{tabular}

(Source: Sinneuau G, et al. 2013²)

treatment with two or more drugs (oral/inhalational drugs) should be considered (Recommendation Class IIa, Evidence Level B). Combined use of intravenous and subcutaneous drugs involves the risk of infection and bleeding from medical devices inserted subcutaneously or intravenously and is therefore of Class IIb indications (Evidence Level C). Shunt closure operation is contraindicated in cases of Eisenmenger's syndrome (Recommendation Class III, Evidence Level C).

The manner of using drugs for treatment of unrepaired shunt-related PAH (not diagnosed as Eisenmenger's syndrome) is basically identical to that for treatment of Eisenmenger's syndrome; there may also be cases in which shunt closure is possible among these cases. The criteria for shunt closure operation are shown in Table $16 .^{8}$ Shunt closure operation should be considered in cases satisfying PVR $<2.3$ Wood Unit (PVRI $<4$ Wood Unit $\cdot \mathrm{m}^{2}$ ) (Recommendation Class IIa, Evidence Level B). Also when medication resulted in favorable responses satisfying the criteria for shunt closure, i.e., PVR $<2.3$ Wood Unit (PVRI $<4$ Wood Unit $\cdot \mathrm{m}^{2}$ ), there is no evidence as to whether shunt closure is a superior choice (although not described in Table 16), and intensive therapy with multiple drugs may occasionally deserve application (Recommendation Class $\mathrm{IIb}$, Evidence Level C). The final judgment as to treatment or shunt closure operation for such cases of unrepaired CHD-PAH should be made at well-experienced facilities, and shunt closure operation should be considered in patients with PVR $<2.3$ Wood Unit (PVRI $<4$ Wood Unit $\cdot \mathrm{m}^{2}$ ) or cases showing improvement in response to treatment to an extent satisfying the criteria and allowing an experienced facility to judge that such operation will be highly beneficial (Recommendation Class IIa, Evidence Level C). In patients with PVR=2.3-4.6 Wood Unit (PVRI 4-8 Wood Unit $\cdot \mathrm{m}^{2}$ ), shunt closure operation should be considered if the operation is expected to be beneficial according to the sufficient evaluation made at a facility with specialists (Recommendation Class IIb, Evidence Level C). According to past reports, the prognosis of adults with residual PAH after shunt closure operation (1, Table 16) is not always favorable compared to that of patients not having undergone shunt repair (patients with or without Eisenmenger's syndrome). ${ }^{285,292}$ Thus, the benefit of shunt closure operation for patients with unrepaired shunt is not assured, and there are concerns about possible risks arising from such an operative procedure. On the other hand, there is a report that shunt closure operation for cases of PAH associated with ASD is expected to be beneficial if this operation is limited to cases satisfying certain criteria, ${ }^{293}$ and more detailed evaluation is expected about the indications of closure in patients with PVRI $\geq 8$ Wood Unit $\cdot \mathrm{m}^{2}$. In general, the judgment as to shunt closure operation for cases of ACHD-PAH is basically contraindicated at facilities other than those providing expert care of ACHD-PAH, regardless of whether the cases satisfy the criteria (Recommendation Class III, Evidence Level C).

Regarding (1) PAH after shunt repair or incidentally accompanied by small shunt (2.1.1, Table 16), the guidelines to IPAH treatment should be followed. Although small shunt closure is basically contraindicated (Recommendation Class III, Evidence Level C), shunt closure operation may be considered in patients with in which pulmonary hypertension has been controlled very well (PVR $<2.3$ Wood Unit or PVRI $<4.0$ Wood Unit $\cdot \mathrm{m}^{2}$ ) and the volumetric load due to the left-to-right shunt through a small hole increases pulmonary ventricle (right ventricle in normal cases) dysfunction or cases in which control of pulmonary hypertension is poor due to PAP elevation and the small shunt can be closed with a less invasive percutaneous approach. However, such a judgment and implementation of the operation should be made at facilities providing expert care of ACHD-PAH (Recommendation Class IIb, Evidence Level C). Closure of the other small shunts is generally contraindicated (Recommendation Class III, Evidence Level C).

(Cardio)pulmonary transplantation is considered in cases of progressive Eisenmenger's syndrome not responding to medication or cases of ACHD-PAH after repair (Recommendation Class IIa, Evidence Level B). However, considering the scant organ supply from donors, the not always favorable prognosis after (cardio)pulmonary transplantation (five-year survival rate about $60 \%$, i.e., $50-75 \%)^{294-297}$ and the open question as to the extent of efficacy expected from combined drug therapy, the judgment as to the timing of registration with the transplantation recipient list has become more difficult after clinical introduction of drugs for PAH treatment.

\section{1.4.5 Pregnancy/Delivery}

Pregnancy and delivery are basically contraindicated in all patients with ACHD-PAH (Recommendation Class III, Evidence Level C). 


\section{I1.5 Drug- and Toxin-Induced Pulmonary Arterial Hypertension}

Many drugs and toxins have been identified as risk factors for the development of PAH. At the Nice Conference in 2013, these factors were divided into the four groups shown in Table $\mathbf{1 7}$ based on evidence for their association

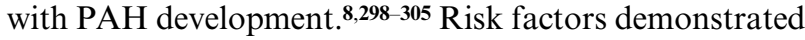
to be associated with the development of PAH by epidemiological studies were considered to be "definitely associated". Risk factors for which there is only a single case control study or several case reports were considered to be "likely associated". Possible risk factors were defined as those with a similar mechanism of action as the above-mentioned factors, but on which there was no study of association. Unlikely risk factors were previously found to not be associated in epidemiological studies. Although the mechanisms of action suggested these factors to be possibly associated, those previously reported to not be associated in epidemiological studies were considered to be "not associated."

Dasatinib, a tyrosine kinase inhibitor (TKI), has been used for many patients with chronic myelogenous leukemia as first-line treatment. The French registry previously reported 9 patients with dasatinib-associated $\mathrm{PAH},{ }^{306} 8$ of whom exhibited clinical and hemodynamic improvement 4 months after discontinuing dasatinib. Moreover, one patient with pulmonary arterial hypertension after treatment with ponatinib, a new TKI, was reported, indicating a possible association of this drug with the development of PAH. ${ }^{307}$

In 2016, several Japanese patients with ulcerative colitis treated by "Seitai", an herbal medicine including natural indigo, were reported to have developed pulmonary hypertension, and the Ministry of Health, Labour and Welfare issued a recommendation for the use of "Seitai" for patients with ulcerative colitis. ${ }^{308}$ Although the causal relationship between the development of PAH and "Seitai" is still under investigation, caution is required when using this herbal medicine.

As stated above, some drugs are risk factors for the development of PAH. As drug-induced pulmonary hypertension may be reversed by discontinuation of the drug, the medical drug history of patients is of the utmost importance for the management of PAH. If drug-induced PAH does not improve after discontinuation of the related drug, targeted treatment is needed.

\section{1.6 PAH Associated With HIV Infection (HIV-Associated PAH)}

\section{Summary}

The prevalence of HIV-associated PAH is low. Basically, this type of PAH is treated in the same manner as IPAH. When drugs for treatment of pulmonary hypertension are used in combination with anti-HIV drugs, sufficient care is needed for drug interactions. Anticoagulant therapy is not recommended.

\subsubsection{Epidemiology and Etiology}

It has been reported that the prevalence of HIV-associated PAH among HIV-infected patients is low $(0.46 \%) .{ }^{15}$ The mechanism for onset of PAH in HIV-infected patients remains unclarified. Because the virus is not detected in the site of plexiform lesions, this type of PAH has been considered as possibly attributable to viral infection-associated inflammation and indirect influence of cytokines such as interleukin (IL)-6, tumor necrosis factor- $\alpha$ (TNF- $\alpha$ ), and platelet-derived growth factor (PDGF). It has been shown that glycoprotein 120, which is involved in the HIV's invasion of macrophages and CD4 lymphocytes stimulates the secretion of endothelin, which induces pulmonary vasoconstriction by its activity targeting human pulmonary endothelial cells. ${ }^{309}$

\subsubsection{Prognosis}

The prognosis of HIV-associated PAH had been quite poor until highly effective anti-retroviral therapy (HAART) and drugs for treatment of pulmonary hypertension were introduced clinically, with the fatality during one year after diagnosis being $50 \% .{ }^{310}$ After introduction of these therapies the prognosis has markedly improved, with the one-year survival rate rising to $88 \%$ and the five-year survival rate exceeding 70\%.311,312 Independent factors associated with poor prognosis for patients with HIV-associated PAH are reported to be $\mathrm{CD}^{+}$lymphocyte count $<200 / \mu \mathrm{L}$ and cardiac index $<2.8 \mathrm{~L} / \mathrm{min} / \mathrm{m}^{2}$. 311

\section{1.6.3 Diagnosis}

Because the prevalence is low, it is not recommended to conduct screening of pulmonary hypertension in symptomfree HIV-infected patients. However, in HIV-infected patients presenting with symptoms, such as unexplained dyspnea, echocardiography should be performed for the purpose of clarifying HIV-associated PAH as well as myocarditis and cardiomyopathy. Right heart catheterization is indispensable for a definite diagnosis of HIV-associated PAH because it can rule out the involvement of left heart disease. Pulmonary vasoreactivity tests are not recommended because the percentage of responders among patients with HIV-associated PAH is low (less than 2\%). ${ }^{\mathbf{1 , 3 1 3}}$

\section{1.6.4 Therapeutic Strategy}

According to the ESC/ERS Guidelines for the Diagnosis and Treatment of Pulmonary Hypertension 2015, the treatment algorithm basically identical to that for IPAH is recommended. ${ }^{1}$ However, when drugs for treatment of pulmonary hypertension are used in combination with protease inhibitors for treatment of HIV, blood levels of the former drugs can be increased by the CYP3A4 inhibitory activity of the latter drugs, possibly elevating the incidence of adverse reactions. Contraindicated combinations are a combination of tadalafil with ritonavir/atazanavir/ indinavir/nelfinavir/saquinavir/darunavir, a combination of sildenafil with ritonavir/indinavir/darunavir, and a combination of riociguat with ritonavir/lopinavir/atazanavir/ indinavir/saquinavir. Also, when drugs requiring caution for combined treatment, rather than drugs contraindicated for combined treatment, are used, sufficient care needs to be taken of possible drug interactions by starting treatment at a low dose level.

Anticoagulant therapy is not recommended for reasons of risk of bleeding, interactions between anticoagulants and anti-HIV drugs, and lack of evidence supporting the usefulness of anticoagulant therapy in cases of HIV-associated PAH. ${ }^{1}$ In patients with HIV-associated PAH, the response to the pulmonary vasoreactivity test is poor and, for this reason, calcium antagonists are not recommended. ${ }^{313}$ In an open-label study, although carried out on a small sample size, 16-week treatment with bosentan in 16 patients with HIV-associated PAH resulted in significant improve- 

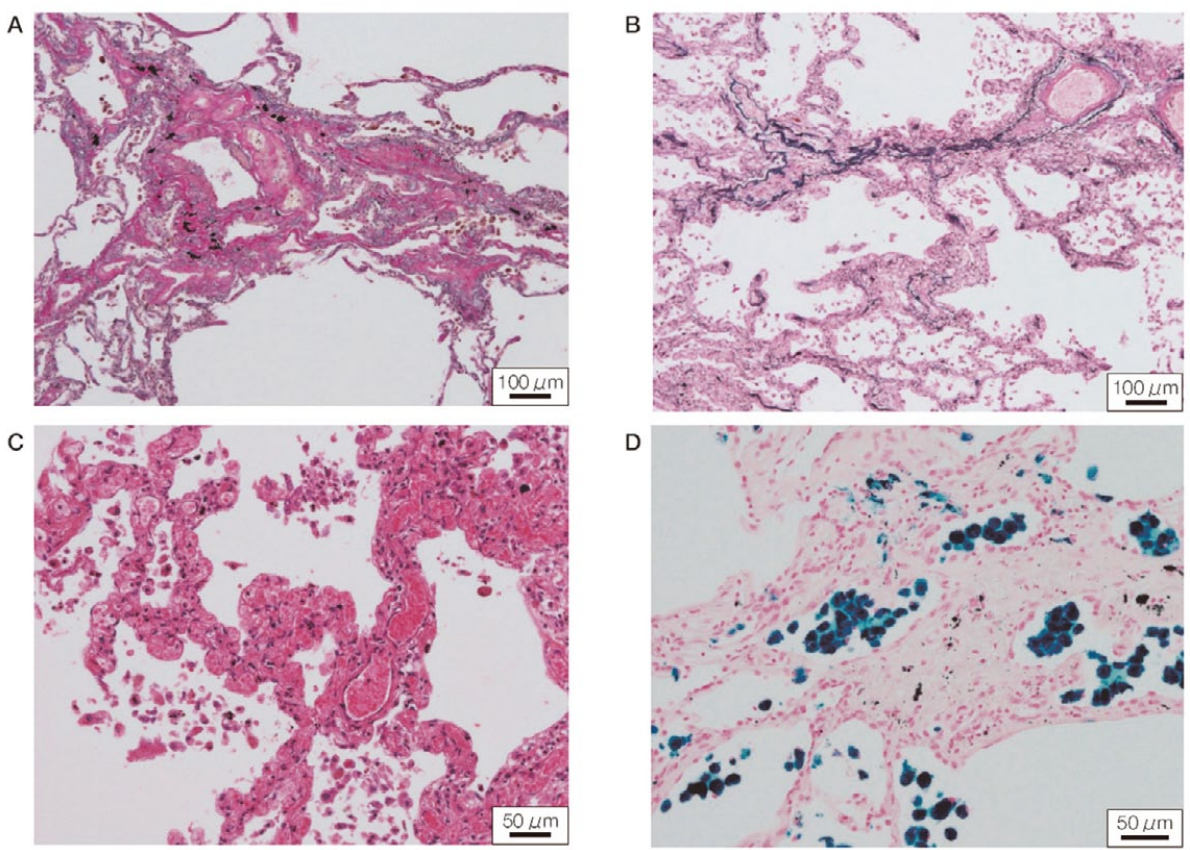

Figure 9. Pathological findings of pulmonary veno-occlusive disease (PVOD). (A) Severe luminal stenosis due to fibrous intimal thickening of the pulmonary vein (EVG staining). (B) Occlusion and collapse of the pulmonary vein of the interlobular septum, surrounded by capillary hyperplasia of alveolar septum (EVG staining). (C) Pulmonary capillary hemangiomatosis-like hyperplasia and dilatation of alveolar wall (HE staining). (D) Macrophages with phagocytosed hemosiderin [hemosiderin-laden macrophages accumulation within the alveolar spaces (Berlin blue staining)].
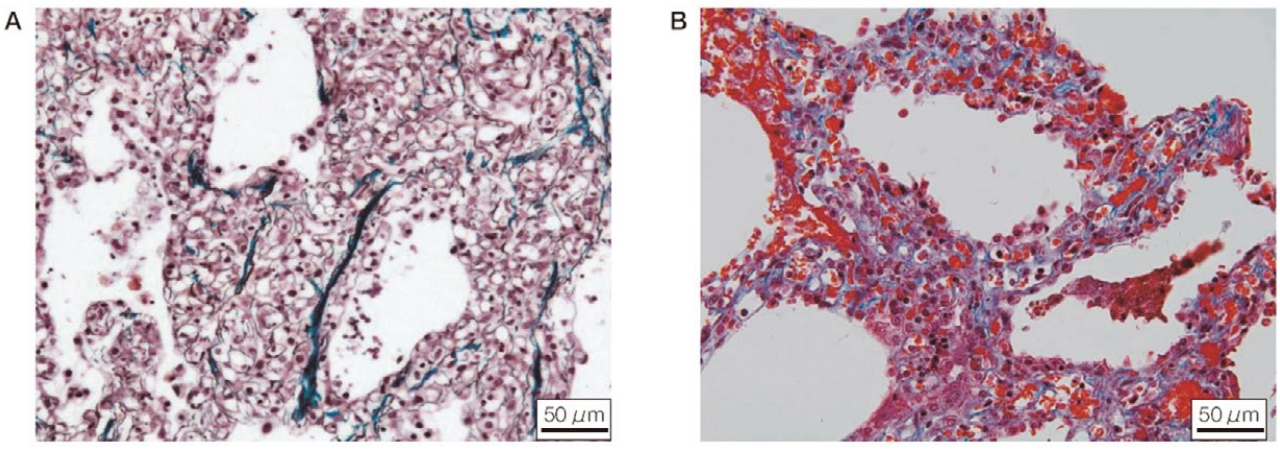

Figure 10. Pathological findings of pulmonary capillary hemangiomatosis (PCH). (A) Capillary proliferation of alveolar septum form 5 or more layers (silver impregnation staining). (B) Alveolar septal thickening due to capillary hyperplasia and dilatation (Masson's trichrome staining).

ment of 6MWD, NYHA functional class, mPAP, cardiac index, PVR, each indicator of echocardiography, and patient's QOL. ${ }^{314}$ Other than these reports, there are case reports suggesting the effectiveness of sildenafil and prostanoid, but no report from large-scale study or analysis has been published. ${ }^{315-317}$

Regarding the efficacy of HAART for HIV-associated $\mathrm{PAH}$, different results have been reported, and no definite conclusion has yet been reached about the efficacy of this therapy. ${ }^{318-320}$

\section{1.7 Pulmonary Veno-Occlusive Disease (PVOD) and/or Pulmonary Capillary Hemangiomatosis (PCH)}

Summary

Both PVOD and PCH are diseases causing pulmonary hypertension due to stenosis and obstruction in pulmonary veins and/or capillaries. These two diseases have many similarities in terms of pathological and clinical features and some patients with these diseases have common 


\begin{tabular}{|l|l|c|}
\hline \multicolumn{2}{|c|}{$\begin{array}{c}\text { Table 18. Treatment of Pulmonary Hypertension Due to Left } \\
\text { Heart Disease (PH-LHD) }\end{array}$} & Recommendation \\
\hline $\begin{array}{l}\text { Treatment of heart failure in } \\
\text { Class }\end{array}$ & $\begin{array}{c}\text { Evidence } \\
\text { Level }\end{array}$ \\
$\begin{array}{l}\text { accordance with the Heart } \\
\text { Failure Treatment Guidelines if } \\
\text { left-sided heart failure is respon- } \\
\text { sible for PH-LHD }\end{array}$ & I & \\
\hline $\begin{array}{l}\text { Treatment of left heart disease } \\
\text { responsible for PH-LHD in } \\
\text { accordance with the guidelines } \\
\text { on the disease concerned }\end{array}$ & I & C \\
\hline $\begin{array}{l}\text { Pulmonary vasodilators aimed } \\
\text { at alleviating or improving the } \\
\text { symptoms/prognosis of patients } \\
\text { with PH-LHD }\end{array}$ & IIb & B \\
\hline $\begin{array}{l}\text { Epoprostenol aimed at improv- } \\
\text { ing the prognosis }\end{array}$ & III & C \\
\hline $\begin{array}{l}\text { Bosentan aimed at improving } \\
\text { the prognosis }\end{array}$ & III & C \\
\hline
\end{tabular}

mutations. In addition, the primary lesion of these two diseases is located at a site different from that of PAH, and these two conditions have the risk of pulmonary edema if PAH-targeted drugs for treatment are used. Because of these features, PVOD and PCH are collectively classified as Group 1' (subtype of Group 1). Their definite diagnosis is based on histopathological findings. For determination of a therapeutic strategy, early clinical diagnosis based on characteristic clinical findings is important.

\section{1.7.1 Epidemiology, Etiology, and Prognosis}

$\mathrm{PVOD} / \mathrm{PCH}$ have been considered to be very rare. However, there is a report that $\mathrm{PVOD} / \mathrm{PCH}$ were detected by postmortem examination in about $10 \%$ of the patients clinically diagnosed with IPAH. ${ }^{321,322}$ The prevalence of PVOD is estimated to be 1 or 2 patients/million. Familial PVOD is diagnosed primarily at younger ages (20s) without sex-related difference. On the other hand, solitary PVOD is often found in males and at higher ages. The prognosis is quite poor, with death from right-sided heart failure or respiratory failure may occur in about 2 years after patients became symptomatic. There are also cases in which the condition aggravates rapidly, leading to death in several months after onset of symptoms. ${ }^{323,324}$

The etiology remains unclarified. However, mutation of the gene encoding the eukaryotic cell translation initiation factor 2 alpha kinase 4 (EIF2AK4) has been reported in cases of PVOD and PCH (familial type and some solitary cases). ${ }^{325,326}$ Furthermore, association of PVOD/PCH with anti-cancer agents (mitomycin, CYC), ${ }^{327,328}$ scleroderma (see 1.2. CTD-PAH [Clinical Classification of Pulmonary Hypertension]), viral infection, smoking, and bone marrow transplantation has been reported.

\section{1.7.2 Pathology}

A pathological feature of PVOD is primary occlusive lesions affecting the peripheral pulmonary veins. ${ }^{20,322}$ Smooth muscles in medial layer of pulmonary vein may thicken similar to muscular arteries (Figure 9A). Fibrous thickening of external layer of pulmonary vein may also occur, resulting in duplication and multilayer external elastic lamina. Following increases in PAP, medial and intimal hyperplasia occurs also on pulmonary arteries, but plexiform lesions seldom appear. Fibrosis from the alveolar septum to the interlobular vein as well as venous obstruction in the interlobular pleura is also seen (Figure 9B). Not only pulmonary vein lesions but also pulmonary capillary hemangiomatosislike lesions, such as sporadic capillary congestion and capillary hyperplasia, are occasionally noted (Figure 9C). Bleeding due to collapse of the dilated capillaries leads to detection of numerous macrophages with phagocytosed hemosiderin in the alveoli. Interstitial edema and lymph duct dilatation are observed (Figure 9D). Lymph node swelling is also seen frequently. ${ }^{329}$

Pulmonary vein obstruction is occasionally seen also in cases of scleroderma or cases after anti-cancer drug treatment, and this is probably attributable to inflammation or fibrosis.

A pathological characteristic of $\mathrm{PCH}$ is capillary hemangioma-like lesions in the alveolar wall and this kind of lesion is often difficult to differentiate from a tumorous lesion. ${ }^{330,331}$ Sporadic capillary congestion as well as capillary hyperplasia differing from that in the intact area is apparent. The alveolar septum thickens as a result of marked capillary hyperplasia (occasionally multilayers growing to form 5 layers or more) (Figure 10). Numerous hemosiderin-laden macrophages are recognized in the alveoli.

\section{1.7.3 Clinical Diagnosis}

A definite diagnosis is based on the histopathological findings described above, but a clinical diagnosis based on characteristic clinical findings is also possible. Lung biopsy is not recommended because of the high risk.

An initial symptom is often shortness of breath or dyspnea on exertion. Compared to $\mathrm{PAH}$, reduction of oxygen saturation at rest and its marked reduction during mild exertion are observed. Other than these, significantly low diffusion capacity of carbon monoxide is also useful in the diagnosis. On chest X-ray, findings, such as ground glass opacity and Kerley's B line, are present. Of the HRCT findings, three signs (thickening of the subpleural septal lines, centrilobular ground glass opacity, and mediastinal lymphadenopathy) are useful in the diagnosis. ${ }^{39} \mathrm{~A}$ ventilation/perfusion lung scan occasionally reveals subsegmental perfusion defects.

Regarding the PVOD/PCH clinical diagnostic criteria and the criteria for official listing of intractable diseases, reference should be made to Chapter IV. "Pulmonary hypertension as an 'intractable disease' listed by Ministry of Health, Labour and Welfare, Appendix 3. Pulmonary veno-occlusive disease/pulmonary capillary hemangiomatosis (Listed Intractable Disease 87)”.

\section{1.7.4 Treatment}

There is no established medical treatment for PVOD or $\mathrm{PCH}$, and lung transplantation (Recommendation Class I, Evidence Level C) is the only radical treatment available. ${ }^{1}$ The effectiveness of PAH-targeted drugs in patients with PVOD/PCH has not been established, and these drugs involve the risk of inducing pulmonary edema. Therefore, treatment with these drugs should be limited to facilities with extensive experience of treatment of pulmonary hypertension. Although there are reports demonstrating that careful treatment with low-dose epoprostenol can be a bridge treatment before transplantation, ${ }^{\mathbf{3 3 0 , 3 3 2}}$ it involves the risk of inducing severe pulmonary edema. Patients with $\mathrm{PVOD} / \mathrm{PCH}$ should be managed in cooperation with 


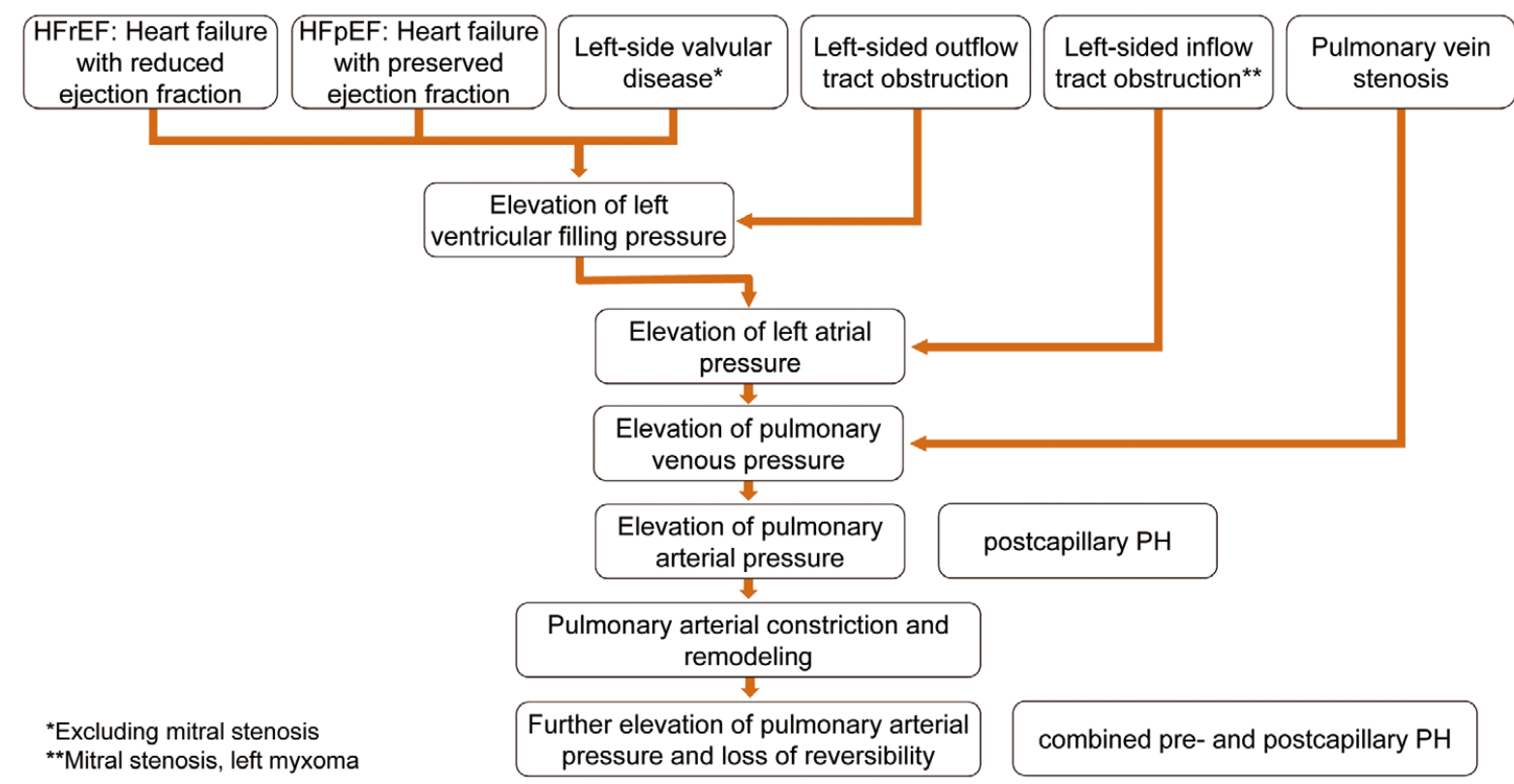

Figure 11. Mechanism for onset of pulmonary hypertension due to left heart disease.

pulmonary hypertension centers with extensive experience in pulmonary hypertension. If the patient desires, registration with the lung transplantation waiting list is necessary soon after diagnosis.

\section{Pulmonary Hypertension Due to Left Heart Disease}

\section{Summary}

In patients with various types of left heart disease involving increases in left ventricular filling pressure or left atrial pressure, transmission of these pressures via pulmonary capillaries to the pulmonary arteries causes pulmonary hypertension. Such "post-capillary" pulmonary hypertension is usually reversible. However, if it is complicated by reactive contraction and remodeling of pulmonary arteries, the disease becomes irreversible. If left heart disease is complicated by pulmonary hypertension, this complication can be a factor for the poor prognosis of heart disease, and this is not exceptional also in cases of heart failure with preserved ejection fraction. A therapeutic strategy for such cases is to perform appropriate treatment to the underlying left heart disease to reduce the left ventricular filling pressure or the left atrial pressure. Treatment with pulmonary vasodilators has also been attempted, but no drug has been shown to be effective. Table $\mathbf{1 8}$ shows the recommendation class and evidence level concerning treatment of pulmonary hypertension due to left heart disease.

\subsection{Definition}

Pulmonary hypertension involving increases in pulmonary venous pressure primarily due to left heart disease was classified as Group 2 (pulmonary hypertension due to left heart disease: PH-LHD) in the Nice Classification (2013).
This is considered the most frequent type of pulmonary hypertension. PH-LHD is subdivided into four types according to the cause; (1) left ventricular systolic dysfunction, (2) left ventricular diastolic dysfunction, (3) valvular heart disease, and (4) congenital/acquired left cardiac inflow tract/outflow tract obstruction. The fourth type is relatively rare, and most cases fall under the first three types.

\section{2.2 Mechanism}

Post-capillary pulmonary hypertension can be caused by various left heart diseases. Left heart diseases that can cause pulmonary venous hypertension include diseases involving increases in the left ventricular filling pressure (e.g., leftsided heart failure) and diseases involving increases in the left atrial pressure due to impaired inflow to the left ventricle (e.g., mitral stenosis and left atrial myxoma). The mechanism for onset of PH-LHD is illustrated in Figure 11.

Left-sided heart failure can be divided into heart failure with reduced ejection fraction (HFrEF) and heart failure with preserved ejection fraction $(\mathrm{HFpEF})$. Both types involve increases in the left ventricular filling pressure, resulting in increases in the left atrial pressure as well. In cases of mitral stenosis or left atrial myxoma, the left ventricular filling pressure does not increase because blood inflow to the left ventricle is impaired, but the left atrial pressure is increased. The increased left atrial pressure is transmitted from the pulmonary vein via the pulmonary capillaries to the pulmonary arteries, resulting in increases in pulmonary artery pressure (PAP).

Pulmonary hypertension at this stage is reversible, and PAP decreases if the left atrial pressure is reduced by treatment. However, if this condition persists long, reactive contraction occurs in the pulmonary artery, resulting in exacerbation of pulmonary hypertension. If pulmonary artery remodeling leads to anatomical changes, pulmonary 


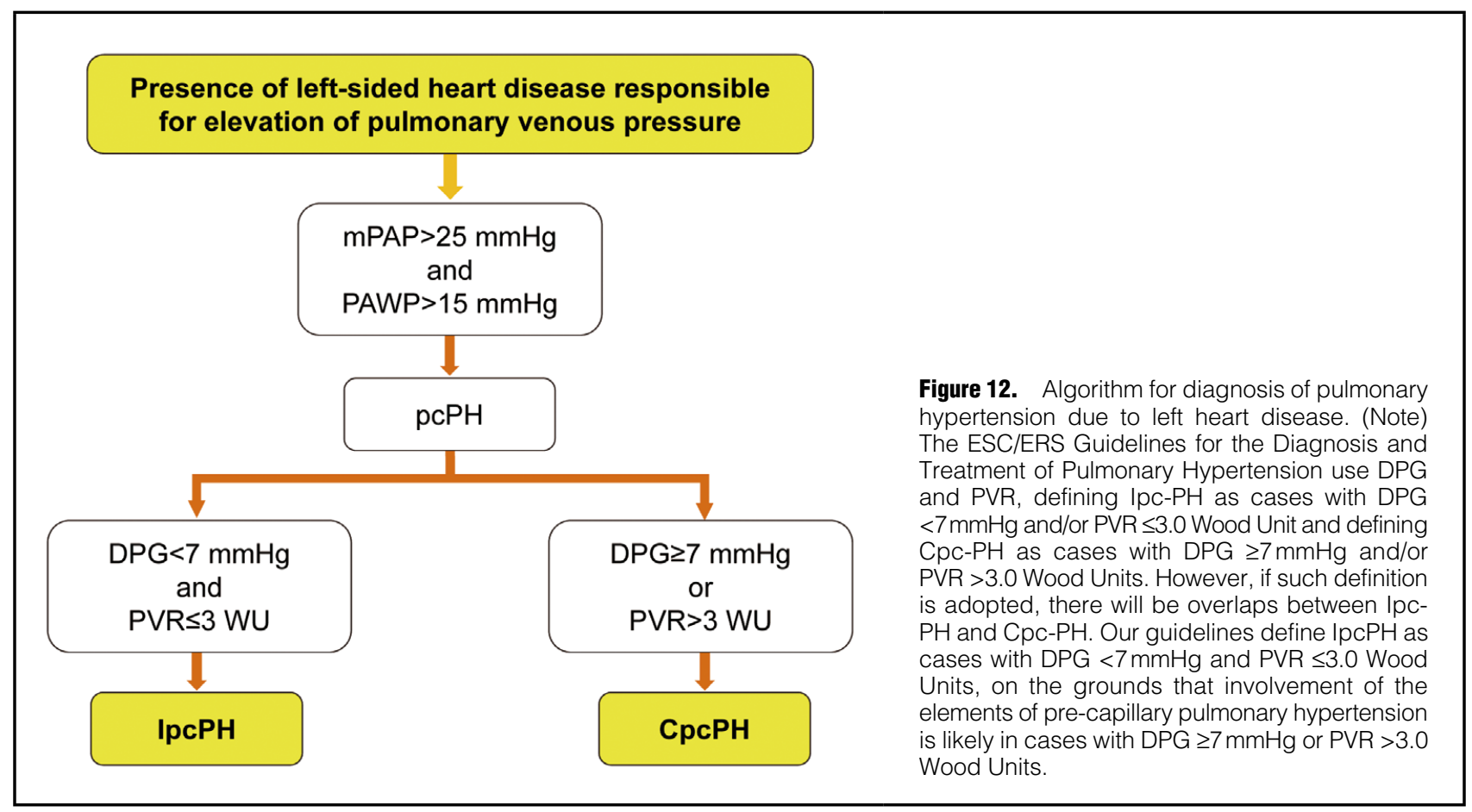

hypertension further increases and become irreversible. Such a state was previously called "out of proportion," meaning that pulmonary hypertension has become too severe to be explained on the basis of increased pulmonary venous pressure alone. Recently, however, the adjective "reactive" has begun to be used to express this condition for distinction from the state called "passive" which simply reflects the pulmonary venous atrial pressure.

In cases of constrictive pericarditis (one of the left heart diseases), increases in PAP does not reflect the pulmonary venous pressure unlike the other left heart diseases. In such cases, the right atrial pressure also increases and the right ventricular diastolic function is insufficient due to impaired inflow to the right heart system, and the reduction in the right ventricular end-diastolic volume reduces the stroke volume and the right ventricular pressure. Thus, the increase in the left atrial pressure is offset by the reduction in right ventricular pressure. As a result, the pulmonary arterial systolic pressure (determined as right ventricular diastolic pressure+right ventricular developed pressure) usually shows no increase or shows relatively mild elevation.

\section{2.3 Epidemiology}

The most frequent type of pulmonary hypertension is pulmonary venous hypertension due to left heart disease. According to the statistics in Western countries, 26-80\% of all patients with left-sided heart dysfunction have been reported to be complicated by pulmonary hypertension ${ }^{333-335}$ Although data in Japan are insufficient, the percentage complicated by pulmonary hypertension was 20-40\% according to the data on right heart catheterization in Japan. ${ }^{336,337}$ If we consider that the estimated number of patients with heart failure in Japan exceeds one million and that most of such patients have left-sided heart failure, the number of patients with heart failure accompanied by pulmonary venous hypertension far exceeds the number of patients accompanied by PAH. Pulmonary hypertension often develops also in patients with $\mathrm{HFpEF}$, a recently increasing disease, ${ }^{335,338}$ and there is a report in Japan demonstrating that pulmonary hypertension with an estimated pulmonary artery systolic pressure $\geq 35 \mathrm{mmHg}$ was observed in $50 \%$ of all patients with HFpEF although it was seen in $43 \%$ of patients with $\mathrm{HFrEF}{ }^{336}$

Since the report by Abramson in 1992, numerous studies demonstrated that PH-LHD is a factor worsening the prognosis of heart failure. ${ }^{333,334,339}$ Although many of the past reports pertained to HFrEF, the role of PH-LDH as a poor prognostic factor has been reported also in cases of HFpEF. ${ }^{335}$

Because pulmonary hypertension also affects the outcome of heart transplantation, ${ }^{333}$ pulmonary hypertension higher than a certain severity level (patients with pulmonary vascular resistance $\geq 6.0$ Wood Unit despite the use of pulmonary vasodilators) is one of the exclusion criteria for determination of the indications of heart transplantation.

\subsection{Etiology/Diagnosis}

Relevant guidelines should be referred to concerning the diagnosis of the left heart disease responsible for pulmonary hypertension. HFpEF is a responsible disease that tends to be frequently overlooked. The diagnosis of HFpEF begins with the clinical finding that heart failure is suspected on the basis of symptoms or brain natriuretic peptide (BNP) despite preserved left ventricular ejection fraction $(50 \%$ or higher). The next step which has been recommended is to exclude cases of non-cardiac disease presenting with features similar to heart failure and then to exclude patients with underlying cardiopulmonary disease, such as congenital heart disease, valvular heart disease, high output heart failure, pericardial disease, and $\mathrm{PAH}$, followed by establishment of the diagnosis of diastolic dysfunction on the basis of left ventricular inflow pattern, pulmonary venous return 
pattern, Doppler ultrasound findings of mitral annular tissue, left atrial dimension or volume, BNP or N-terminal proBNP (NT-proBNP), pulmonary artery wedge pressure (measured by right heart catheterization), and other data.

Pulmonary hypertension associated with connective tissue disease primarily refers to $\mathrm{PAH}$, which is classified as Group 1 as a rule. In cases of systemic sclerosis, care needs to be taken of the fact that left ventricular diastolic function is often impaired, ${ }^{340}$ and post-capillary associated with HEpEF may be also involved.

The requirements for diagnosis of post-capillary pulmonary hypertension associated with left heart disease are: (1) pulmonary hypertension (mean PAP $>25 \mathrm{mmHg}$ ) revealed by right heart catheterization; and (2) increases in pulmonary artery wedge pressure $(>15 \mathrm{mmHg})$.

PH-LHD in most cases has a severity comparable to the degree of increase in pulmonary venous pressure (postcapillary pulmonary hypertension: $\mathrm{pc} \mathrm{PH}$ ), but there are cases presenting with pulmonary hypertension that cannot be fully explained by increases in pulmonary venous pressure (associated with left-sided heart failure) alone. In such cases, the involvement of pre-capillary features due to remodeling of the pulmonary artery itself is likely, and the disease concept "combined pre- and postcapillary $\mathrm{PH}$ $(\mathrm{CpcPH})$ " is used (approximately corresponding to the previously used term "out-of-proportion pulmonary hypertension"). For distinction between pcPH and $\mathrm{CpcPH}$, transpulmonary pressure gradient (TAG: mean PAP-mean pulmonary artery wedge pressure) has been used with the cut-off level $12 \mathrm{mmHg}$. Recently, diastolic pressure gradient (DPG: diastolic pulmonary pressure-pulmonary artery wedge pressure) $\leq 7 \mathrm{mmHg}$ has also begun to be used for such distinction. There is no consensus about which of these two criteria should be used, but there is a study suggesting that DPG probably allows better identification of a poor prognosis group than does TPG. ${ }^{337}$ The ESC/ ERS Guidelines for the Diagnosis and Treatment of Pulmonary Hypertension 2015 recommend the use of DPG and pulmonary vascular resistance (PVR), stating that a diagnosis of isolated postcapillary $\mathrm{PH}$ (IpcPH) should be made if DPG $<7 \mathrm{mmHg}$ and/or PVR $\leq 3.0$ Wood Unit and that a diagnosis of $\mathrm{CpcPH}$ should be made if DPG $\geq 7 \mathrm{mmHg}$ and/or PVR $>3.0$ Wood Unit. ${ }^{1}$ The algorithm for diagnosis of PH-LHD according to our guidelines is illustrated in Figure 12.

Pulmonary arterial capacitance (PAC) is calculated by dividing the stroke volume by the pulmonary artery pulse pressure. In patients with PH-LHD, reduction of PAC has been reported as a poor prognostic factor, ${ }^{341-344}$ and it can be useful as an indicator for prediction of the outcome.

\subsection{Treatment}

\section{2.5.1 Treatment of Responsible Disease}

For treatment of PH-LHD, what should be done first is to perform appropriate treatment to the responsible left heart disease so that the pulmonary venous pressure is reduced. In cases possibly attributable to left ventricular dysfunction, priority should be given to treatment of cardiac dysfunction in accordance with the acute heart failure treatment guidelines or the chronic heart failure treatment guidelines. In addition, diuretics and pulmonary vasodilators are most effective in alleviating pulmonary hypertension. In patients with heart failure due to left ventricular systolic dysfunction, treatment with angiotensin-converting enzyme (ACE)

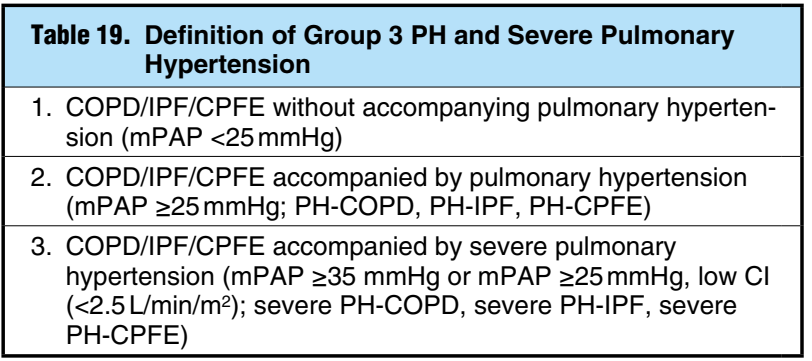

(Source: Prepared based on Galiè N, et al. 2016¹)

inhibitors (angiotensin II receptor blockers (ARB) if not tolerable with ACE inhibitors), $\beta$-blockers, and aldosterone antagonists is recommended to improve prognosis. Also from long-term perspectives, success in reversing the left ventricular remodeling by these drugs is expected to result in reduction of left ventricular filling pressure and reduction of PAP. Similar efficacy is expected also of cardiac resynchronization therapy (CRT) which is a non-drug therapy. In cases of $\mathrm{HFpEF}$, diuretics and pulmonary vasodilators are useful in reducing congestion and the left ventricular filling pressure, but no drug improving the prognosis or no treatment method reducing pulmonary hypertension by improvement of left ventricular diastolic function has been identified.

\section{2.5.2 Drugs for Treatment of Pulmonary Hypertension in PH-LHD Patients}

Numerous clinical studies have been conducted on drugs for treatment of pulmonary hypertension in patients with left-sided heart failure. Although not limited to patients with left-sided heart failure complicated by pulmonary hypertension, a study on the prostanoid "epoprostenol" (FIRST Study) 345 and a study on endothelin receptor antagonists (ERAs) (Enable Study) ${ }^{346}$ have been carried out. Both studies failed to confirm the prognosis-improving effects, yielding only negative results. As a clinical study of ERA in patients with CpcPH, MELODY-1 Study was completed in 2015. This study was designed to evaluate the efficacy of macitentan by analysis of two primary endpoints (percentage of patients experiencing bodily fluid pool and aggravation of NYHA functional class). ${ }^{347}$ Its results have not yet been reported. A clinical study has been conducted also on drugs acting on the nitrogen monoxide (NO)-cyclic guanosine monophosphate (cGMP) signal transduction system, revealing that sildenafil improved the hemodynamics and left ventricular diastolic function in patients with HFpEF complicated by pulmonary hypertension (pulmonary artery systolic pressure $\geq 40 \mathrm{mmHg}$ ). ${ }^{348}$ However, subsequent placebo-controlled randomized studies yielded negative results. ${ }^{349,350}$ Thus, the efficacy of treatment targeting the pulmonary hypertension in PH-LHD patients has not yet been clarified.

\section{Pulmonary Hypertension Due to Lung Diseases and/or Hypoxia}

\section{Summary}

Pulmonary hypertension due to lung disease and/or hypoxia (Group 3) involves conditions such as chronic obstructive pulmonary disease (COPD), interstitial lung disease, other 


\begin{tabular}{|c|c|c|}
\hline \multicolumn{3}{|c|}{$\begin{array}{l}\text { Table 20. Recommendation Class and Evidence Level } \\
\text { Concerning Treatment of Pulmonary Hypertension } \\
\text { Due to Lung Diseases }\end{array}$} \\
\hline Recommendation & $\begin{array}{l}\text { Recommendation } \\
\text { Class }\end{array}$ & $\begin{array}{l}\text { Evidence } \\
\text { Level }\end{array}$ \\
\hline $\begin{array}{l}\text { Long-term oxygen therapy for } \\
\text { cases accompanied by severe } \\
\text { respiratory failure (arterial } \\
\text { oxygen tension } \leq 55 \text { Torr) }\end{array}$ & 1 & A \\
\hline $\begin{array}{l}\text { Lung transplantation for severe } \\
\text { cases }\end{array}$ & 1 & B \\
\hline $\begin{array}{l}\text { Appropriate treatment of } \\
\text { underlying disease }\end{array}$ & Ila & $\mathrm{C}$ \\
\hline $\begin{array}{l}\text { Vasodilator therapy for cases of } \\
\text { pulmonary hypertension } \\
\text { assuming the form of PAH } \\
\text { (mPAP } \geq 35 \mathrm{mmHg} \text { ) }\end{array}$ & Ila & B \\
\hline $\begin{array}{l}\text { Long-term oxygen therapy for } \\
\text { cases of semi-respiratory failure } \\
\text { accompanied by hypoxia during } \\
\text { sleep/on exertion }\end{array}$ & Ilb & $B$ \\
\hline $\begin{array}{l}\text { Pulmonary vasodilator therapy } \\
\text { for cases of pulmonary } \\
\text { hypertension due to lung } \\
\text { disease (mPAP }<35 \mathrm{mmHg} \text { ) }\end{array}$ & Ilb & $B$ \\
\hline $\begin{array}{l}\text { Riociguat therapy for cases of } \\
\text { pulmonary hypertension due to } \\
\text { idiopathic interstitial pneumonia }\end{array}$ & III & $B$ \\
\hline $\begin{array}{l}\text { Ambrisentan therapy for cases } \\
\text { of pulmonary hypertension due } \\
\text { to idiopathic pulmonary fibrosis }\end{array}$ & III & $B$ \\
\hline
\end{tabular}

lung diseases consisting of a mixture of restrictive and obstructive disorders, alveolar hypoventilation syndrome, sequelae to pulmonary tuberculosis, and sleep-disordered breathing. The actual prevalence of accompanying pulmonary hypertension of each type is unknown. However, cases of pulmonary hypertension associated with COPD, idiopathic pulmonary fibrosis (IPF), or combined pulmonary fibrosis and emphysema (CPFE) are encountered frequently during clinical practice.

Chronic interstitial pneumonia, such as COPD and IPF, has a phenotype characterized by complication by pulmonary arterial hypertension $(\mathrm{PAH})$ or the presence of accompanying pulmonary hypertension. In these patients with pulmonary hypertension, restriction of exercise ability is seen, thus presenting with a feature of pulmonary hypertension that can be associated with poor prognosis. However, both respiratory system lesions and cardiovascular lesions can be related to restriction of exercise and prognosis, making it difficult to make a clinical judgment as to which of these lesions is involved and to which extent it is involved. The disease concept "CPFE" requires reevaluation/review, but patients with both emphysema and interstitial pneumonia are more likely to have the complication by pulmonary hypertension compared to patients with COPD or IPF. For the diagnosis of pulmonary hypertension or PAH associated with COPD or interstitial pneumonia, echocardiography and right heart catheterization are indispensable, as is the case with groups of pulmonary hypertension other than Group 3. However, for distinction between Group 1 pulmonary hypertension (PAH) accompanied by respiratory system lesions and Group 3 pulmonary hypertension (pulmonary hypertension associated with lung diseases), comprehensive evaluation is needed at a facility providing expert care of both pulmonary hypertension and respiratory

\begin{tabular}{|c|c|c|}
\hline \multicolumn{3}{|c|}{$\begin{array}{l}\text { Table 21. Recommendation Class and Evidence Level } \\
\text { Concerning Treatment of Pulmonary Hypertension } \\
\text { Due to Sleep-Disordered Breathing }\end{array}$} \\
\hline Recommendation & $\begin{array}{l}\text { Recommendation } \\
\text { Class }\end{array}$ & $\begin{array}{l}\text { Evidence } \\
\text { Level }\end{array}$ \\
\hline $\begin{array}{l}\text { CPAP therapy for cases of sleep- } \\
\text { disordered breathing satisfying } \\
\text { the criteria for indications }\end{array}$ & Ila & B \\
\hline $\begin{array}{l}\text { NPPV therapy for cases of } \\
\text { severe nocturnal hypoventilation }\end{array}$ & Ila & B \\
\hline $\begin{array}{l}\text { Weight loss guidance for obese } \\
\text { patients }\end{array}$ & $\mathrm{Ilb}$ & C \\
\hline $\begin{array}{l}\text { Oxygen therapy at home for } \\
\text { patients with severe respiratory } \\
\text { failure (arterial oxygen tension } \\
\leq 55 \text { Torr) difficult for CPAP/ } \\
\text { NPPV therapy }\end{array}$ & $\mathrm{Ilb}$ & $\mathrm{C}$ \\
\hline $\begin{array}{l}\text { Pulmonary vasodilator therapy } \\
\text { for patients with pulmonary } \\
\text { hypertension of PAH (mPAP } \\
\geq 35 \mathrm{mmHg} \text { ) }\end{array}$ & Ilb & $\mathrm{C}$ \\
\hline
\end{tabular}

\begin{tabular}{|c|c|c|}
\hline \multicolumn{3}{|c|}{$\begin{array}{l}\text { Table 22. Recommendation Class and Evidence Level } \\
\text { Concerning Treatment of Pulmonary Hypertension } \\
\text { Due to Alveolar Hypoventilation Syndrome }\end{array}$} \\
\hline Recommendation & $\begin{array}{l}\text { Recommendation } \\
\text { Class }\end{array}$ & $\begin{array}{l}\text { Evidence } \\
\text { Level }\end{array}$ \\
\hline NPPV therapy & Ila & B \\
\hline $\begin{array}{l}\text { Oxygen therapy at home for } \\
\text { patients with severe respiratory } \\
\text { failure (arterial oxygen tension } \\
\leq 55 \text { Torr) difficult for CPAP/ } \\
\text { NPPV therapy }\end{array}$ & $\mathrm{Ilb}$ & $\mathrm{C}$ \\
\hline
\end{tabular}

diseases.

For patients with Group 3 pulmonary hypertension, it is necessary to avoid using the term "out of proportion" over time. Use of Table $\mathbf{1 9}$ as the definition of pulmonary hypertension associated with COPD, IPF, or CPFE is recommended (evaluation should be made at rest, performing supplementary oxygen inhalation as needed). Table 19 is based on the ESC/ERS Guidelines for the Diagnosis and Treatment of PH 2015. Severe pulmonary hypertension is defined as patients with mean pulmonary artery pressure (mPAP) $\geq 35 \mathrm{mmHg}$. This may require further modification over time, depending on the outcome of future studies. ${ }^{1}$ The numerals and signs of inequality used in Table 19 are not supported by a definite evidence.

Severe pulmonary hypertension includes also some patients with chronic lung disease suspected of involving pulmonary vascular remodeling similar to PAH (reduction in reserve circulatory function rather than reduced reserve ventilatory ability leading to restriction of exercise function may be a major feature of such cases). Clinical signs suggesting poor prognosis of severe pulmonary hypertension include: (1) dyspnea on exertion more marked than anticipated from the pulmonary function test data, (2) reduction of pulmonary diffusion function more marked than anticipated from the routine lung function test data, and (3) marked reduction of arterial oxygen tension during exercise. When studies are conducted on the efficacy of drugs for treatment of pulmonary hypertension which are currently not covered by health insurance if used for patients with pure Group 3 pulmonary hypertension, focus 


\begin{tabular}{|c|c|c|}
\hline $\begin{array}{l}\text { Criteria favoring } \\
\text { Group } 1 \text { (PAH) }\end{array}$ & Parameter & $\begin{array}{l}\text { Criteria favoring Group } 3 \text { (pulmonary } \\
\text { hypertension due to lung disease) }\end{array}$ \\
\hline $\begin{array}{l}\text { Normal or mildly impaired } \\
\% F V_{1}>60 \% \text { (COPD) } \\
\% F V C>70 \% \text { (IPF) }\end{array}$ & Ventilatory function & $\begin{array}{l}\text { Moderate to very severe impairment } \\
\% \text { FEV }_{1}<60 \% \text { (COPD) } \\
\% F V C<70 \% \text { (IPF) }\end{array}$ \\
\hline $\begin{array}{l}\text { Absence of or only modest airway or parenchymal } \\
\text { abnormalities }\end{array}$ & $\begin{array}{l}\text { High-resolution CT } \\
\text { scan }\end{array}$ & Characteristic airway and/or parenchymal abnormalities \\
\hline $\begin{array}{l}\text { Features of exhausted circulatory reserve } \\
\text { Preserved breathing reserve } \\
\text { Reduced oxygen pulse } \\
\text { Low } \mathrm{CO} / \mathrm{VO}_{2} \text { slope } \\
\text { Mixed venous oxygen saturation at lower limit } \\
\text { No change or decrease in } \mathrm{PaCO}_{2} \text { during exercise }\end{array}$ & $\begin{array}{l}\text { Circulatory/ventilatory } \\
\text { reserve }\end{array}$ & $\begin{array}{l}\text { Features of exhausted ventilatory reserve } \\
\text { Reduced breathing reserve } \\
\text { Normal oxygen pulse } \\
\text { Normal CO/ } \dot{\mathrm{O}}_{2} \text { slope } \\
\text { Mixed venous oxygen saturation above lower limit } \\
\text { Increase in } \mathrm{PaCO}_{2} \text { during exercise }\end{array}$ \\
\hline
\end{tabular}

(Source: Seeger W, et al. 2013 ${ }^{365}$ )

should be made on severe pulmonary hypertension at first. However, referral to facilities with specialists is necessary for care of individual patients.

Recommendation class and evidence level are shown in Tables 20 to 22 concerning treatment of pulmonary hypertension due to lung diseases, pulmonary hypertension due to sleep-disordered breathing, and pulmonary hypertension due to alveolar hypoventilation syndrome.

\subsection{COPD}

\subsubsection{Pathophysiology}

The prevalence of pulmonary hypertension due to COPD (COPD-PH) varies depending on the COPD stage and the definition of pulmonary hypertension. In studies of patients with stage IV COPD according to the Global Initiative for Chronic Obstructive Lung Disease (GOLD) classification, the percentage of patients with mPAP $\geq 20 \mathrm{mmHg}$ was $90 \%$ at maximum. Among these patients, the highest percentage was in patients not satisfying the criteria for pulmonary hypertension, with mPAP at rest being about $20-25 \mathrm{mmHg}$, and only $3-5 \%$ of the patients had $\mathrm{mPAP} \geq 35 \mathrm{mmHg}$ (severe pulmonary hypertension). ${ }^{\mathbf{2 1}, 351}$ The histopathological changes in pulmonary vascular lesions seen in COPD patients are associated with the severity of pulmonary hypertension and these changes in severe cases resemble those of PAH patients. ${ }^{352}$ In COPD patients, increases in mPAP are often seen even during moderate exercise, and such increase in MPAP are related to reduction of pulmonary vascular distensibility and vascular recruitment ability.

Progression of COPD-PH is usually slow (less than $1 \mathrm{mmHg}$ increase per year). ${ }^{353}$ However, the expression of pulmonary hypertension in COPD patients is one of the factors determining the prognosis of COPD even when pulmonary hypertension is moderate and the MPAP and pulmonary vascular resistance (PVR), which are indicators of pulmonary hypertension severity, correlate inversely with the survival rate. ${ }^{351,354,355}$ The five-year survival rate of COPD patients presenting with pulmonary hypertension ( $\mathrm{mPAP} \geq 25 \mathrm{mmHg}$ ) is $36 \%$. Thus, indicators of pulmonary hemodynamics are more important as prognostic factors than are forced expiratory volume in one second $\left(\mathrm{FEV}_{1}\right)$ and indicators of gas exchange. ${ }^{355}$

\section{3.1.2 Diagnosis/Evaluation of Pulmonary Hypertension in COPD Patients}

Echocardiography is the diagnostic imaging modality first used for noninvasive diagnosis of pulmonary hypertension in COPD patients. However, this technique is known to result in poor study frequency in cases of advanced COPD because of the inability to perform sufficient observation due to the increased air contained in the lungs (overswollen lungs). When echocardiography data for diagnosis of pulmonary hypertension were compared with right heart catheterization (RHC) data in patients with respiratory disease, the positive predictive value was $32 \%$ and the negative predictive value was $93 . .^{356,357}$ In cases of severe COPD-PH, increases in serum levels of brain natriuretic peptide (BNP) or N-terminal proBNP (NT-proBNP) are seen, but the detective sensitivity of these parameters in cases of moderate COPD-PH is low. ${ }^{358}$

The RHC test is the gold standard for diagnosis of pulmonary hypertension. This test should be conducted in all patients with chronic lung disease who satisfy the following criteria:

(1) Evaluation in preparation for lung transplantation is judged necessary;

(2) The progression of clinical aggravation/exercise function restriction cannot be explained by progression of ventilatory disorder alone;

(3) The progression of gas exchange disorder (reduction in oxygen tension $\left[\mathrm{PO}_{2}\right]$ ) is not proportionate to the progression of ventilatory disorder;

(4) Accurate evaluation of prognosis is judged necessary;

(5) Severe pulmonary hypertension is suspected on the basis of noninvasive evaluation and further use of drugs for treatment or participation in clinical study or registry study is considered;

(6) Suspected of having left ventricular diastolic or systolic dysfunction.

Evaluation using both RHC and exercise load test may allow identification of whether the reduction of reserve ventilatory capacity is predominant or the reduction of reserve circulatory ability due to $\mathrm{PH}$ is predominant in the patient's condition. ${ }^{359}$

Among lung diseases, the term "in proportion" pulmonary hypertension is based on the assumption that remodeling of the lung parenchyma architecture in the presence of respiratory disease accompanied by hypoxia can simul- 


\begin{tabular}{|c|c|c|c|}
\hline $\begin{array}{l}\text { Underlying Lung } \\
\text { Disease }\end{array}$ & $\begin{array}{c}\text { mPAP }<25 \mathrm{mmHg} \\
\text { at Rest }\end{array}$ & $\begin{array}{c}\mathrm{mPAP} \geq 25 \text { and } \\
<35 \mathrm{mmHg} \text { at Rest }\end{array}$ & $\begin{array}{c}\text { mPAP } \geq 35 \mathrm{mmHg}^{*} \\
\text { at } \text { Rest }^{\star}\end{array}$ \\
\hline \multirow{2}{*}{$\begin{array}{l}\text { COPD with } \mathrm{FEV}_{1} \geq 60 \% \text { of } \\
\text { predicted } \\
\text { IPF with FVC } \geq 70 \% \text { of predicted } \\
\text { CT: absence of or only very } \\
\text { modest airway or parenchymal } \\
\text { abnormalities }\end{array}$} & No PH & PH classification uncertain & $\begin{array}{l}\text { PH classification uncertain: discrimination } \\
\text { between } \mathrm{PAH} \text { (group 1) with concomitant } \\
\text { lung disease or } \mathrm{PH} \text { caused by lung disease } \\
\text { (group } 3 \text { ) }\end{array}$ \\
\hline & $\begin{array}{l}\text { No PAH treatment } \\
\text { recommended }\end{array}$ & $\begin{array}{l}\text { No data currently support treatment } \\
\text { with PAH-approved drugs }\end{array}$ & $\begin{array}{l}\text { Refer to a center with expertise in both } \mathrm{PH} \\
\text { and chronic lung disease }\end{array}$ \\
\hline \multirow{2}{*}{$\begin{array}{l}\text { COPD with } \mathrm{FEV}_{1}<60 \% \text { of } \\
\text { predicted } \\
\text { IPF with FVC }<70 \% \text { of predicted } \\
\text { Combined pulmonary fibrosis } \\
\text { and emphysema on CT }\end{array}$} & No PH & PH-COPD, PH-IPF, PH-CPFE & $\begin{array}{l}\text { Severe PH-COPD, severe PH-IPF, severe } \\
\text { PH-CPFE }\end{array}$ \\
\hline & $\begin{array}{l}\text { No } \mathrm{PAH} \text { treatment } \\
\text { recommended }\end{array}$ & $\begin{array}{l}\text { No data currently support treatment } \\
\text { with PAH-approved drugs }\end{array}$ & $\begin{array}{l}\text { Refer to a center with expertise in both } \mathrm{PH} \\
\text { and chronic lung disease for individualized } \\
\text { patient care because of poor prognosis; } \\
\text { randomized controlled trials required }\end{array}$ \\
\hline
\end{tabular}

*Lower PA pressures may be clinically significant in COPD/DPLD patients with depressed cardiac index or right ventricular dysfunction. (Source: Seeger W, et al. 2013365)

taneously cause loss of the total vascular cross-sectional area, resulting in increases in PVR. On the other hand, the term "out of proportion" pulmonary hypertension indicates that the severity of pulmonary hypertension is higher than anticipated from the degree of lung architecture remodeling due to respiratory disease, and this is based on the following hypotheses: (1) chronic respiratory disease triggers pulmonary vascular remodeling, which can progress without direct involvement of pulmonary dysfunction; (2) pulmonary hypertension in patients with chronic respiratory disease can develop "incidentally" regardless of the disease stage and can progress independently from the stage of accompanying respiratory disease. At the Nice Conference, however, the following possibilities were suggested: (1) pulmonary hypertension develops only after $80 \%$ or more of the intact lung structure is lost; and (2) any condition with $\mathrm{mPAP} \geq 25 \mathrm{mmHg}$ may be called "out of proportion." By discussions about these possibilities at the meeting, consensus was reached about the necessity of avoiding the use of this term.

\section{3.1.3 Severe Pulmonary Hypertension in COPD Patients}

The category "severe pulmonary hypertension" refers to patients who have parenchymal disease of the respiratory system and are suspected of undergoing severe pulmonary vessel remodeling. In COPD patients, the category "severe pulmonary hypertension" refers to patients with circulatory disorders that can markedly aggravate the exercise function already reduced by obstructive ventilatory disorder. Such cases accounted for up to $1 \%$ of the 1,218 patients with pulmonary emphysema enrolled in the National Emphysema Treatment Trial (NETT). ${ }^{\mathbf{3 6 0}}$

According to a study published in the journal Chest in 2012, patients with COPD being $\mathrm{mPAP} \geq 40 \mathrm{mmHg}$ showed reduction of reserve circulatory ability during exercise (reduction in both mixed venous oxygen saturation and gradient of cardiac output/oxygen consumption), whereas the respiratory reserve was maintained (reduction in arterial carbon dioxide tension $\left[\mathrm{PaCO}_{2}\right] .{ }^{359}$ On the other hand, patients with COPD but without pulmonary hypertension or with moderate pulmonary hypertension (mPAP: $31 \mathrm{mmHg}$ ) showed restricted exercise function due to ventilatory disorder (reduction in respiratory reserve during exercise and increases in arterial $\mathrm{PaCO}_{2}$ ), whereas the circulatory reserve was maintained. ${ }^{359}$ Compared to pulmonary hypertension-free COPD patients, patients with severe
COPD (mPAP $\geq 40 \mathrm{mmHg}$ ) had higher $\mathrm{FEV}_{1}$ and lower 6-minute walk distance (6MWD). The circulatory disorder in patients with severe PH-COPD restricted the exercise function further, and this should be taken into consideration when attempting to perform appropriate treatment on the basis of accurate assessment of the condition. ${ }^{21}$

In past clinical trials/studies in patients with IPAH/ HPAH, "absence of lung/airway or lung parenchyma disease" was usually adopted as an exclusion criterion for selection of subjects, whereas patients with mild to moderate obstructive ventilatory disorders were included in the trials/ studies. ${ }^{361-364}$ In the largest-scale study among these studies (171 patients with IPAH, mean age 45 , mean PVR 1,371 dyne $\cdot \mathrm{sec} \cdot \mathrm{cm}^{-5}$ ), the mean $\mathrm{FEV}_{1}$ was $83 \%$ of the predicted value and the $\mathrm{FEV}_{1} /$ vital capacity (VC) ratio was $76 \%$, with the $\mathrm{FEV}_{1} / \mathrm{VC}$ ratio lowering $70 \%$ in $22 \%$ of all patients. ${ }^{364}$ Following these results, the parameters of lung function test in the below-shown range were adopted as exclusion criteria for randomized controlled trials of PAH patients.

- Lung volume $<60-70 \%$ of predicted value

- $\mathrm{FEV}_{1}<55-88 \%$ of predicted value

- $\mathrm{FEV}_{1} /$ forced vital capacity (FVC) $<50-70 \%$.

Lung disease (particularly COPD) is a common disease, and the expression of PAH in patients with lung disease is not always an outcome of Group 3 pulmonary hypertension but can develop incidentally. The criteria for differential diagnosis for classification of patients with lung disease accompanied by pulmonary hypertension into Group 1 (PAH) or Group 3 (pulmonary hypertension due to lung disease) are summarized in Table $23 .{ }^{365}$ In patients with uncertain features, referral to facilities providing expert care is needed.

\subsubsection{Long-Term 0xygen Therapy (LTOT) for COPD-PH}

The lung disease responsible for pulmonary hypertension should be treated in accordance with the existing guidelines. Of the guidelines on respiratory diseases available at present, no guidelines recommend treatment methods focusing on the vascular lesions of lung disease (excluding long-term oxygen therapy). In patients with COPD presenting with arterial oxygen tension $\left(\mathrm{PaO}_{2}\right)<60 \mathrm{mmHg}$, it has been estimated that long-term oxygen therapy (LTOT) can improve the prognosis and that improvement in pulmonary hemodynamics rather than respiratory function is related to improvement in prognosis. ${ }^{\mathbf{2 4}}$ A study reported in 2016 
by the LOTT Research Group demonstrates that the length of time until death or first hospitalization of COPD patients was not extended by 24-hour oxygen therapy continued for a long period of time (1-6 years) in COPD patients with oxygen saturation $\left(\mathrm{SpO}_{2}\right)$ at rest being $89-93 \%$ or by long-term oxygen therapy during exercise/ sleep in COPD patients presenting with hypoxia on exertion, although the study did not include analysis by the presence/absence or severity of pulmonary hypertension. ${ }^{366}$ These results suggest that long-term oxygen therapy is of little benefits except when it is performed in COPD-PH patients presenting with respiratory failure $\left(\mathrm{PaO}_{2}\right.$ at rest $<60 \mathrm{mmHg}$ ).

\section{\3.1.5 Vasoactive Drugs in COPD Patients (Taking Into Consideration Appropriate Benefit/Risk Ratio of Drugs for Treatment of Pulmonary Hypertension)}

It is difficult to use pulmonary vasodilators in COPD patients without causing aggravation of gas exchange. ${ }^{367}$ Inhalational prostanoid preparations can reduce $\mathrm{MPAP}$ and PVR without aggravating the gas exchange in COPD-PH patients, but there are long-term clinical study results. ${ }^{368}$ Regarding the use of bosentan in patients with COPD accompanied by mild pulmonary hypertension, a smallscale randomized placebo-controlled study revealed aggravation of gas exchange and poor improvement in maximum oxygen consumption, exercise function, and QOL in the bosentan treatment group. ${ }^{369}$ However, another small-scale clinical study demonstrated improvement of exercise function following treatment with bosentan in COPD-PH patients. ${ }^{370}$ Thus at present, there is very little data that can strongly support the efficacy of endothelin receptor antagonists (ERAs) on pulmonary hemodynamics and exercise tolerability in COPD-PH patients.

When sildenafil was administered for a short period of time to COPD-PH patients, some patients showed aggravation of gas exchange although their hemodynamics were improved. ${ }^{371}$ One-month treatment with sildenafil in COPD patients without complication by pulmonary hypertension resulted in no change in 6MWD or maximum oxygen consumption $\left(\dot{\mathrm{VO}}_{2}\right)$, but their $\mathrm{PaO}_{2}$ and QOL aggravated. ${ }^{372,373}$ Also in a randomized placebo-controlled study of COPD patients without severe pulmonary hypertension, sildenafil treatment during pulmonary rehabilitation failed to improve exercise tolerability. ${ }^{374}$ However, in a small-scale randomized placebo-controlled study, long-term sildenafil treatment in patients with COPD complicated by severe pulmonary hypertension resulted in reduction of pulmonary artery pressure (PAP) and improvement of 6MWD. ${ }^{375}$ Thus, no consensus has yet been reached about the efficacy of sildenafil in patients with severe COPD-PH. Furthermore, there is little evidence concerning the long-term efficacy of sildenafil in treating COPD patients without severe pulmonary hypertension.

In the ASPIRE Registry Study, involving a series of untreated COPD-PH patients $(n=101)$, comparison was made between 42 patients with severe COPD-PH (mPAP $\geq 40 \mathrm{mmHg}$ ) and patients with mild to moderate COPD-PH (mPAP $<40 \mathrm{mmHg}$ ). In the severe COPD-PH group, pulmonary vasodilator therapy did not improve the survival rate as a whole, but improvement of survival rate was seen in patients showing improvement of WHO functional class or $20 \%$ or more reduction of PVR after treatment. ${ }^{376,377}$ It is an open issue to identify characteristics of patients who may respond well to treatment.

\section{3.1.6 Recommendation of Treatment for COPD-PH}

Long-term randomized controlled trial may be the only means of collecting highly reliable data supporting the usefulness of drugs for treatment of pulmonary hypertension in patients with severe pulmonary hypertension or chronic obstructive/restrictive lung disease. Implementation of such a study is awaited. Because the responses to treatment in such a clinical study may differ depending on the underlying disease, it is necessary to conduct investigation separately for patients with obstructive lung disease and patients with restrictive lung disease during such a clinical study. Regarding the pulmonary hypertension patients associated with chronic lung diseases listed below, it is necessary to classify the patients as shown in Table $\mathbf{2 4}$ on the basis of the lung function test (detecting airway/ parenchymal lung disease), cardiopulmonary exercise load test, clinical findings, CT-based evidence, and pulmonary hypertension severity so that individual patients may receive the treatment recommended for each group. ${ }^{365}$

1. First, patients for whom the diagnosis of pulmonary hypertension is clinically suspected (patients without severe obstructive/restrictive ventilatory disorder revealed by the lung function test and without evident airway/ lung disease visualized by CT scan) are identified. There are difficulties in determining which PAH complicated by lung disease (Group 1 pulmonary hypertension) or pulmonary hypertension attributable to lung disease (Group 3 pulmonary hypertension) is present in a given patient. These patients should therefore be referred to expert care facilities so that they can receive detailed examinations such as high-resolution $\mathrm{CT}$, hemodynamic evaluation, accurate respiratory function tests, and cardiopulmonary exercise load tests.

2. Signs of both chronic lung disease and pulmonary hypertension are seen in many IPF patients with $\% \mathrm{FVC}<70 \%$ and COPD patients with $\% \mathrm{FEV}_{1}<60 \%$ (obstructive/ restrictive ventilatory disorder) and $25 \leq \mathrm{mPAP}<35 \mathrm{mmHg}$. To date, there is no data supporting the effectiveness of drugs for treatment of pulmonary hypertension for these groups of patients. Because the restriction of exercise function in these patients is primarily based on ventilatory dysfunction rather than circulatory dysfunction, the effectiveness of the drugs for treatment of pulmonary hypertension is questionable in these patients. Use of pulmonary vasodilators can impair gas exchange particularly in COPD patients. Although we do not rule out the possibility that pulmonary vascular lesions affect the progression of the disease or that vascular lesions become the target of treatment in the future, no controlled clinical study focusing on this point has been reported.

3. The prognosis is poor for the group of patients with evident obstructive/restrictive ventilatory disorder and presenting with severe PH-COPD, severe PH-IPF, or severe PH-CPFE (mPAP $\geq 35 \mathrm{mmHg}$ ). To provide care tailored to the features of individual patients of this group, referral to facilities with specialists who can manage pulmonary hypertension and chronic lung disease is needed. Hemodynamic analysis of these patients resulted in the following two suggestions: (1) restriction of maximum $\dot{\mathrm{VO}}_{2}$ and restriction of physical function are affected by the state in which cardiac output is lower than normal and/or the increase in cardiac output during the exercise load test is insufficient; and 
(2) increase in the after-load on the right heart system is a major factor involved in hemodynamic aggravation. It is necessary to carry out a randomized controlled study involving this group of patients. In such patients, it is essential to consider using the drugs for treatment of pulmonary hypertension as well as conducting monitoring of gas exchange function $\left(\mathrm{PaO}_{2}, \mathrm{PaCO}_{2}\right)$ and participating in a prospective registry study in view of establishing evidence for the future. Regarding the influence on gas exchange, both aggravation (by suppression of hypoxic vascular contractive responses) and improvement (increases in mixed venous oxygen saturation by pulmonary vasodilation at the normal oxygen level site and increase in cardiac output by medication) are possible.

4. In patients with terminal stage obstructive/restrictive lung disease or with complication by such a disease, the drugs for treatment of pulmonary hypertension have not been recommended in view of possible shortening of mean life expectancy. However, the current indications for the drugs for treatment of pulmonary hypertension may change over time under the recent circumstances in which usefulness has been shown in the use of membrane type artificial lungs as a bridge to transplantation $^{378}$ or in the long-term use of a noninvasive respirator at home, which is expected to extend the mean life expectancy of such patients. It is necessary to conduct a controlled study involving patients with terminal stage obstructive/restrictive lung disease or pulmonary hypertension, requiring support with a respirator or a membrane type artificial lung, for the purpose of examining whether the drugs for treatment of pulmonary hypertension can contribute to improving the exercise function and QOL, extending the time until clinical aggravation, improving the survival data and maintaining the condition during the pre-transplantation bridge period.

\subsection{Interstitial Lung Disease}

\section{| 3.2.1 Epidemiology/Prognosis}

Interstitial lung disease involves diverse features and types, and epidemiological data concerning pulmonary hypertension with complicated interstitial lung disease are scant. However, the percentage having been complicated by $\mathrm{PH}$ at the time of diagnosis has been reported to be $5-15 \%$ for patients with IPF 379,380 and about $30-60 \%$ for patients with severe IPF. ${ }^{381-385}$ The pulmonary hypertension complication rate among the Japanese patients with idiopathic interstitial pneumonia having undergone right heart catheterization has been reported to be about $3 \% .{ }^{386}$

Regarding the prognosis, complication by pulmonary hypertension has been shown in domestic and overseas studies to be a poor prognostic factor for interstitial lung disease including IPF. ${ }^{358,387-390}$ According to the recent report by Tanabe et al., the three-year survival rate was as low as $35.7 \%$ for Japanese patients with interstitial lung disease accompanied by severe pulmonary hypertension. ${ }^{390}$ Also in a British registry study, the prognosis was poorer for patients with interstitial lung disease complicated by pulmonary hypertension than for patients with COPD-PH. ${ }^{213}$

As illustrated above, interstitial lung disease is an important prognosis determinant for pulmonary hypertension, and it is essential during clinical practice to consider that the prognosis is particularly poor in cases in which interstitial lung disease is accompanied by severe pulmonary hypertension.

\subsubsection{Pathophysiology}

Factors responsible for complication of interstitial lung disease by pulmonary hypertension include pulmonary vascular constriction associated with hypoxia, arteriolar/ capillary compression and obstruction (reduction in pulmonary vascular bed) associated with lung parenchymal disorder, and vascular wall remodeling. ${ }^{391-393}$ There is also a report that pulmonary vein lesions are involved in pulmonary hypertension, which complicates IPF, ${ }^{394}$ a finding important in understanding the pathophysiology and use of pulmonary vasodilators.

\section{| 3.2.3 Diagnosis}

As in other groups of pulmonary hypertension, this group of pulmonary hypertension is diagnosed on the basis of the RHC test findings of mPAP $\geq 25 \mathrm{mmHg}$. Signs and symptoms frequently seen include severe shortness of breath and hypoxia associated with the accompanying lung disease. If symptoms difficult to explain on the basis of lung disease are present or aggravation of such symptoms is noted in patients with lung disease, complication by pulmonary hypertension needs to be considered. Noninvasive tests useful in the diagnosis of this condition include blood BNP and NT-proBNP measurement, respiratory function tests (including pulmonary diffusing ability assessment), and echocardiography. However, the ability of diagnosing pulmonary hypertension with complicated interstitial lung disease is low with echocardiography, ${ }^{395}$ and general assessment by combining this test with the respiratory function test, and exercise tolerability test is essential. The RHC test should be considered in cases in which it is considered to be useful in a definite diagnosis of pulmonary hypertension or determining appropriate therapeutic strategy.

\subsubsection{Interstitial Lung Disease Complicated by Severe Pulmonary Hypertension}

Marked increases in PAP are seen in about $20 \%$ of patients with interstitial lung disease complicated by pulmonary hypertension, ${ }^{\mathbf{3 8 4}, 390}$ and this change has been attracting close attention from the viewpoint of pathophysiology, diagnosis, and treatment. As in cases of COPD-PH, severe pulmonary hypertension in patients with interstitial lung disease is defined as $\mathrm{mPAP} \geq 35 \mathrm{mmHg}$ or $\mathrm{mPAP} \geq 25 \mathrm{mmHg}$ and cardiac index $(\mathrm{CI})<2.5 \mathrm{~L} / \mathrm{min} / \mathrm{m}^{2} 2$ (Table 19). Characteristic clinical findings include discrepancy between symptoms and respiratory function, marked hypoxia, a large alveolar gas-arterial oxygen tension gradient $\left(\mathrm{A}-\mathrm{aDO}_{2}\right)$, and reduced exercise tolerability. ${ }^{396}$ Furthermore, the importance of distinction from PAH in cases of severe pulmonary hypertension with complicated interstitial lung disease is described in the ESC/ERS Guidelines for the Diagnosis and Treatment of PH 2015.2,365 Regarding the diagnosis and treatment of severe cases, particularly treatment with pulmonary vasodilators, it is recommended to consider and perform diagnosis/treatment at facilities well experienced with management of both lung disease and pulmonary hypertension.

\subsubsection{Treatment}

In cases of pulmonary hypertension-complicated interstitial lung disease presenting with hypoxia, oxygen therapy 
should be considered. If the patient presents with symptoms of right-sided heart failure, such as edema, low salt diet, sedation, and use of diuretics are considered. Although steroid therapy, immunosuppressive therapy, and antifibrosis therapy have also been attempted corresponding to various types of interstitial lung disease, there is no sufficient data concerning their efficacy on accompanying pulmonary hypertension or safety. If the condition resists medical treatment and is severe and progressive, lung transplantation is considered.

\section{3.2.6 Efficacy and Safety of Drugs for Treatment of Pulmonary Hypertension}

The efficacy of the drugs in the treatment of pulmonary hypertension has not been proven in patients with pulmonary hypertension with complicated interstitial lung disease. Particularly because the two recent studies (placebocontrolled multicenter double-blind studies) demonstrated lack of efficacy of bosentan and riociguat or a high incidence of adverse events, it is generally not recommended to use the drugs for treatment of pulmonary hypertension in patients with interstitial lung disease complicated by pulmonary hypertension. ${ }^{2,365}$ The reports cited below are important in considering the usefulness and safety of drugs for treatment of pulmonary hypertension in interstitial lung disease or pulmonary hypertension with complicated interstitial lung disease.

\section{a. Multicenter Prospective Double-Blind Studies in Patients With Interstitial Lung Disease Complicated by Pulmonary Hypertension \\ i. Bosentan}

When 60 patients with interstitial lung disease complicated by pulmonary hypertension were treated with bosentan for 16 weeks, no significant alleviation or improvement was noted in symptoms, functional class, or PVR (BPHIT Study). ${ }^{397}$

\section{ii. Riociguat}

A study was begun to evaluate the efficacy and safety of riociguat in patients with idiopathic interstitial pneumonia complicated by pulmonary hypertension, but it was discontinued in 2016 because of a high incidence of death and severe adverse events in the drug treatment group (NCT02138825) (RISE-IIP).

\section{b. Observational Study}

\section{i. Phosphodiesterase 5 (PDE5) Inhibitors}

In analysis of the prognosis using the data from a retrospective observational study involving 101 patients with lung disease complicated by severe pulmonary hypertension (including 19 patients with interstitial pneumonia complicated by pulmonary hypertension), the prognosis was better in patients treated with PDE5 inhibitors than in untreated patients. Also in analysis by underlying lung disease, the prognosis of interstitial pneumonia, collagen diseaseassociated interstitial pneumonia, and CPFE was better in the PDE5 inhibitor treatment group (multicenter prospective registry study JRPHS in patients with pulmonary hypertension due to respiratory disease). ${ }^{390}$

\section{c. Multicenter Prospective Placebo-Controlled Double-Blind Studies in Patients With IPF \\ i. Sildenafil}

In 180 patients with severe IPF, 12-week treatment with sildenafil did not improve exercise tolerability (STEPIPF). ${ }^{398}$ However, the study revealed efficacy of sildenafil on shortness of breath and QOL, and sub-analysis of the echocardiographic data from that study demonstrated improvement of exercise tolerability and QOL following sildenafil treatment in patients with right ventricular hypertrophy/dilatation. ${ }^{399}$

\section{ii. Bosentan}

In 158 patients with $\mathrm{PF}$, 12-month treatment with bosentan did not improve exercise tolerability (BUILD-1). ${ }^{400} \mathrm{~A}$ subsequent evaluation involving a larger number of patients $(n=616)$ also failed to reveal the effects of bosentan in suppressing IPF aggravation or death (BUILD-3).401

\section{iii. Ambrisentan}

In 492 patients with IPF, the percentage of patients showing disease aggravation was higher in the ambrisentan treatment group than in the placebo group (hazard ratio $1.74, \mathrm{P}=0.01$ ), and the study was discontinued in 2013 (ARTEMISIPF). ${ }^{402}$ Similar results were obtained also from sub-analysis of the data from the same study by presence/absence of pulmonary hypertension. Following these results, the prospective double-blind study of ambrisentan in pulmonary hypertension-complicated IPF patients was also discontinued (ARTEMIS-PH [NCT00879229]).

\section{iv. Macitentan}

In 178 patients with IPF, treatment with macitentan did not significantly improve the respiratory function (FVC) (MUSIC Study). ${ }^{403}$

All of these studies involved patients with IPF and none of them was designed to directly evaluate the efficacy or safety of drugs for treatment of pulmonary hypertension in patients with pulmonary hypertension. However, considering that the patients studies included patients with pulmonary hypertension, the results from these studies are useful as the information when considering drugs for treatment of pulmonary hypertension in patients with interstitial lung disease complicated by pulmonary hypertension.

\subsubsection{Perspectives for the Future}

At present, there is no single treatment showing evident effects on pulmonary hypertension in patients with interstitial lung disease when performed separately. However, since the pathophysiology and response to treatment can vary in some types of this disease (e.g., severe pulmonary hypertension), the ESC/ERS Guidelines for the Diagnosis and Treatment of Pulmonary Hypertension 2015 also describe that the use of drugs for treatment of pulmonary hypertension should be considered, if other clinical indicators and adverse influence of lung disease are taken into account, in a subset of patients satisfying the criteria of severe pulmonary hypertension (PAH phenotype) among all cases of Group 3 pulmonary hypertension. ${ }^{2}$ Thus, it is necessary to clarify the pathophysiological features that may lead to PAP elevation and to confirm the treatment method by prospective studies.

Regarding Japanese patients, on the other hand, there are reports that even mild pulmonary hypertension may have poor prognosis if it is accompanied by IPF. $\mathbf{3 8 0 , 4 0 4}$ Therefore, accurate diagnosis of mild pulmonary hypertension and evaluation of the usefulness of therapeutic intervention in such cases are open issues over time. 


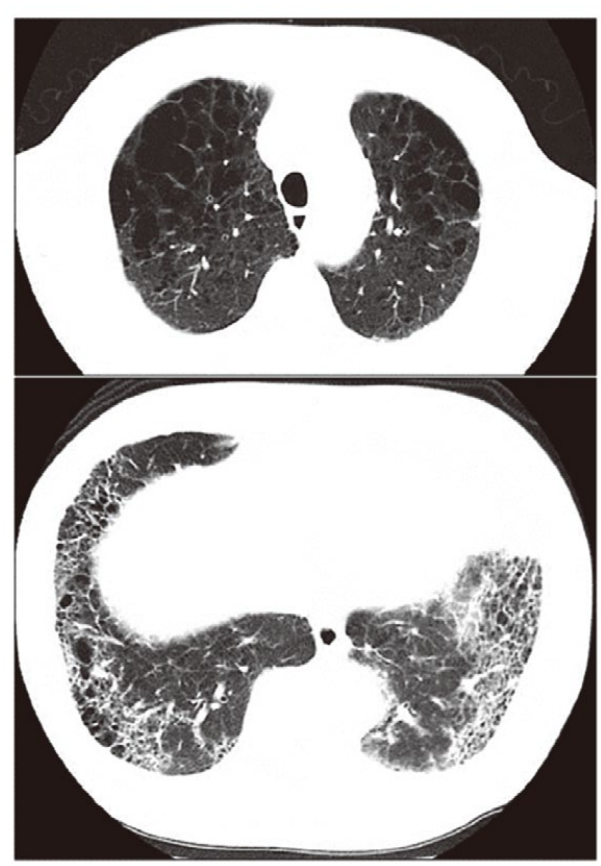

Figure 13. Chest $\mathrm{CT}$ images of a patient with combined pulmonary fibrosis and emphysema (CPFE).

Furthermore, in a multicenter double-blind study of patients with severe IPF suspected of being accompanied by pulmonary hypertension, the efficacy and safety of sildenafil treatment added to pirfenidone therapy are being evaluated (NCT02951429), attracting close attention for insight into a new direction of treatment.

\section{3.3 Other Lung Diseases Involving Mixture of Restrictive and Obstructive Disorders}

CPFE is the most important disease of this category. The disease entity "CPFE" was proposed in 2005 by Cottin et al. as a group of diseases involving centrilobular pulmonary emphysema of upper lung field predominance and fibrosis of lower lung field predominance visualized by CT scan. ${ }^{31}$ Its characteristic clinical findings include: (1) relatively low severity of obstructive/restrictive ventilatory disorders, (2) marked reduction of the pulmonary diffusing ability, (3) marked hypoxia, and (4) frequent complication by pulmonary hypertension. ${ }^{405}$ "Other lung diseases involving a mixture of restrictive and obstructive disorders" represented by CPFE have been described as one of the underlying lung diseases of Group 3 pulmonary hypertension since the Dana Point Classification.

\section{3.3.1 Epidemiology and Pathophysiology}

According to a report by Cottin et al., the percentage of patients with estimated pulmonary artery systolic pressure $>45 \mathrm{mmHg}$ among all cases of CPFE is as high as $47 \%$ (at the time of diagnosis) or 55\% (if cases during follow-up are included). ${ }^{31}$ According to an observational study in Japan, on the other hand, complication by pulmonary hypertension was seen in about $17 \%$ of all cases of CPFE. ${ }^{386}$ Regarding prognosis, Cottin et al. reported that the median survival period for pulmonary hypertension-complicated cases $\left(\mathrm{PVR} \geq 485 \mathrm{dynes} \cdot \mathrm{sec} \cdot \mathrm{cm}^{-5}\right)$ was 6.6 months. ${ }^{405}$ Also in Japan, the prognosis of pulmonary hypertensioncomplicated CPFE cases was poorer than that of patients with other lung diseases complicated by pulmonary hypertension. ${ }^{390}$ However, the diagnostic criteria for CPFE have not yet been established, and the underlying disease and severity level vary greatly among different reports, thus requiring caution in interpretation of epidemiological and prognosis data. There are many open questions also concerning the pathophysiology of this disease. There is a report indicating the presence of venous lesions in patients with CPFE, resembling the cases of pulmonary hypertension with complicated IPF or scleroderma, ${ }^{406}$ and this finding may be considered as important when discussing about the clinical signs, response to treatment and prognosis of this condition.

\section{3.3.2 Diagnosis}

The diagnosis of CPFE should be based on a general assessment of the above-mentioned clinical features. Of the clinical features, chest CT findings are particularly important. Typical CT images are shown in Figure 13.

However, there is no clear-cut diagnostic criteria about which consensus has been reached, and the diagnosis of CPFE has been made on the basis of the judgment by individual physicians and facilities, taking into account the above-mentioned clinical features. As in the diagnosis of pulmonary hypertension complicating other lung diseases, the diagnosis of pulmonary hypertension complicating CPFE is established by right heart catheterization after assessment of the possibility of pulmonary hypertension with echocardiography or other tests.

\section{| 3.3.3 Treatment}

At present, no treatment method showing evident effects on CPFE-complicated pulmonary hypertension is available. Resembling the approach to treatment of COPD or interstitial lung disease, treatment of CPFE is attempted by oxygen therapy, sedation, low salt diet, use of diuretics, assist to ventilation, and removal of aggravating factors.

\section{3.3.4 Efficacy and Safety of Drugs for Treatment of Pulmonary Hypertension}

There is no evidence supporting the usefulness of the drugs for treatment of pulmonary hypertension in the treatment of CPFE-complicated pulmonary hypertension, and the use of such drugs is usually not recommended. ${ }^{2}$ However, retrospective studies suggesting the presence of responders to the drugs for treatment of PAH have been sporadically reported. In Japan, Tanabe et al. noted better prognosis of CPFE-complicated pulmonary hypertension cases following treatment with PDE5 inhibitors compared to the untreated group. ${ }^{390}$

In two overseas observational studies, on the other hand, patients with CPFE-complicated pulmonary hypertension failed to respond to the drugs for treatment of pulmonary hypertension. ${ }^{377,405}$ Other than these reports, there are a few cases reports dealing with the efficacy and safety of the drugs for treatment of pulmonary hypertension in CPFEcomplicated pulmonary hypertension cases. ${ }^{407-409}$

\section{3.3.5 Perspectives and Open Issues for the Future}

The greatest issue is how to establish the disease concept and the diagnostic criteria. The clinical features of CPFE proposed by Cottin et al. are useful in grouping patients 
Table 25. Diagnostic Criteria in the Alveolar Hypoventilation Syndrome Diagnostic Guidelines

\section{A. Symptoms}

C. Differential diagnosis

\section{Genetic test}

1. Severe sleep disorders (e.g., tendency to insomnia and nocturnal waking) and resulting daytime hypersomnia

2. Signs of right-sided heart failure (e.g., shortness of breath at rest or upon exertion and systemic edema)

3. Symptoms associated with daytime reduction of activity

1. Chronic marked hypercapnia $\left(\mathrm{PaCO}_{2}>45\right.$ Torr) revealed by arterial blood gas analysis Phenotype A: Primarily presenting with hypoventilation/hypoxia during sleep at night Phenotype B: Primarily presenting with apnea during sleep at night

2. Arterial blood gas analysis: If chronic respiratory failure $\left(\mathrm{PaO}_{2} \leq 60 \mathrm{Torr}\right)$ is suggested, combined use of $\mathrm{HOT}$ is considered

3. Phenotype (A or B) is determined by overnight polysomnography

Distinguished from the following diseases.

1. Organic lung diseases: COPD, idiopathic interstitial pneumonia, bronchiectasis COPD may be diagnosed in patients presenting with obstructive ventilatory disorder ( $\left.F E V_{1} / F V C<70 \%\right)$. Patients with COPD occasionally present with apnea or hypoventilation during sleep at night

Possible complication by alveolar hypoventilation syndrome needs to be considered in cases of mild to moderate COPD (\%FEV $1 \geq 50 \%$ ) with $\mathrm{PaCO}_{2}>50$ Torr. Respiratory function test is indispensable for the diagnosis of this disease

2. Sleep apnea syndrome (SAS)

If apnea-hypopnea index (AHI) determined by polysomnography is $\geq 5$, a diagnosis of sleep-disordered breathing (SDB) is made. A diagnosis of ASA is made in patients with AHI $\geq 5$ accompanied by signs and symptoms while awake or patients with $\mathrm{AHI} \geq 15$ regardless of the presence/absence of symptoms

In cases of SAS presenting with $\mathrm{PaCO}_{2}>52.5$ Torr (severity grade 2 or higher) while awake, complication by alveolar hypoventilation syndrome is considered. Polysomnography and arterial blood gas analysis while awake are indispensable for the diagnosis of this condition

3. Neuromuscular diseases: Myasthenia gravis

The steps for ruling out drug-induced respiratory center suppression, respiratory muscle paralysis, and other diseases, such as neuromuscular diseases, are taken. It is also necessary to rule out organic lesions of the central nervous system associated with respiratory center abnormalities on the basis of findings from diagnostic imaging and neurological findings

1. $P H O X 2 B$ gene mutation

PHOX2B mutation has been reported for phenotype $\mathrm{A}$, but its significance has not been established (particularly in adults)

Unknown about phenotype B

\section{$<$ Categories of diagnosis>}

- Definite: Satisfying 2 items or more of $A+B-1$, ruled out as to the diseases listed in $C$, and evident as to phenotype (A or B) by test B-3

- Probable: Satisfying 1 item of $A+B-1$, ruled out as to the diseases listed in C, but difficult to judge phenotype (A or B) by test B-3

- Possible: Satisfying B-1, ruled out as to the diseases listed in C, but difficult to judge phenotype (A or B) (polysomnography not performed)

(Source: Prepared based on Tatsumi K. 2015410)

Table 26. Severity Rating According to the Alveolar Hypoventilation Syndrome Management Guidelines

\begin{tabular}{|c|c|c|c|c|}
\hline \multirow[b]{2}{*}{ Severity } & \multirow{2}{*}{$\begin{array}{l}\text { Symptom } \\
\text { Shortness of } \\
\text { breath mMRC }\end{array}$} & \multicolumn{2}{|c|}{ Arterial blood gas analysis } & \multirow{2}{*}{$\begin{array}{l}\text { Treatment status } \\
\text { CPAP/NPPV/HOT }\end{array}$} \\
\hline & & $\mathrm{PaCO}_{2}$ & $\mathrm{PaO}_{2}$ & \\
\hline 1 & $\geq 1$ & $>45$ Torr & \multirow{2}{*}{ Not questioned } & Not questioned \\
\hline 2 & \multirow{3}{*}{$\geq 2$} & \multirow{2}{*}{$\begin{array}{l}\text { A: }>50 \text { Torr } \\
\text { B: }>52.5 \text { Torr }\end{array}$} & & Treatment with CPAP/NPPV needs to be continued \\
\hline 3 & & & $\leq 70$ Torr & $\begin{array}{l}\text { Treatment with CPAP/NPPV/HOT needs to be } \\
\text { continued }\end{array}$ \\
\hline 4 & & A, B: >55 Torr & \multirow{2}{*}{$\leq 60$ Torr } & \multirow{2}{*}{ Treatment with NPPV/HOT needs to be continued } \\
\hline 5 & $\geq 3$ & A, B: $>60$ Torr & & \\
\hline
\end{tabular}

The highest severity level satisfied by all items (symptoms, arterial blood gas analysis, and treatment status) is adopted as the level for a given case. If any item falls under several severity levels, judgment should be based on the other items.

HOT may be discontinued if the condition improves (including the condition during night) in response to treatment.

$\mathrm{A}$ and $\mathrm{B}$ in the item $\mathrm{PaCO}_{2}$ mean phenotype $\mathrm{A}$ and $\mathrm{B}$.

The medical care expense subsidy program covers the patients in whom the severity level of symptoms is lower than a certain level according to the criteria given above but continuation of high-cost treatment is needed.

$\leq$ Modified MRC (mMRC) grading system for shortness of breath $>$

0: Shortness of breath appears only during hard exercise.

1: Shortness of breath appears when walking rapidly on a flat road or walking on a gently rising slope.

2: Because of shortness of breath, the walking speed is lower than others of the same generation or walking is sometimes stopped while walking on a flat road at one's own pace.

3: Shortness of breath causes walking on a flat road to be stopped after walking about $100 \mathrm{~m}$ or for several minutes.

4: Severe shortness of breath makes it impossible to go outdoors, or shortness of breath appears even during dressing/undressing.

(Source: Prepared based on Tatsumi K. 2015410) 

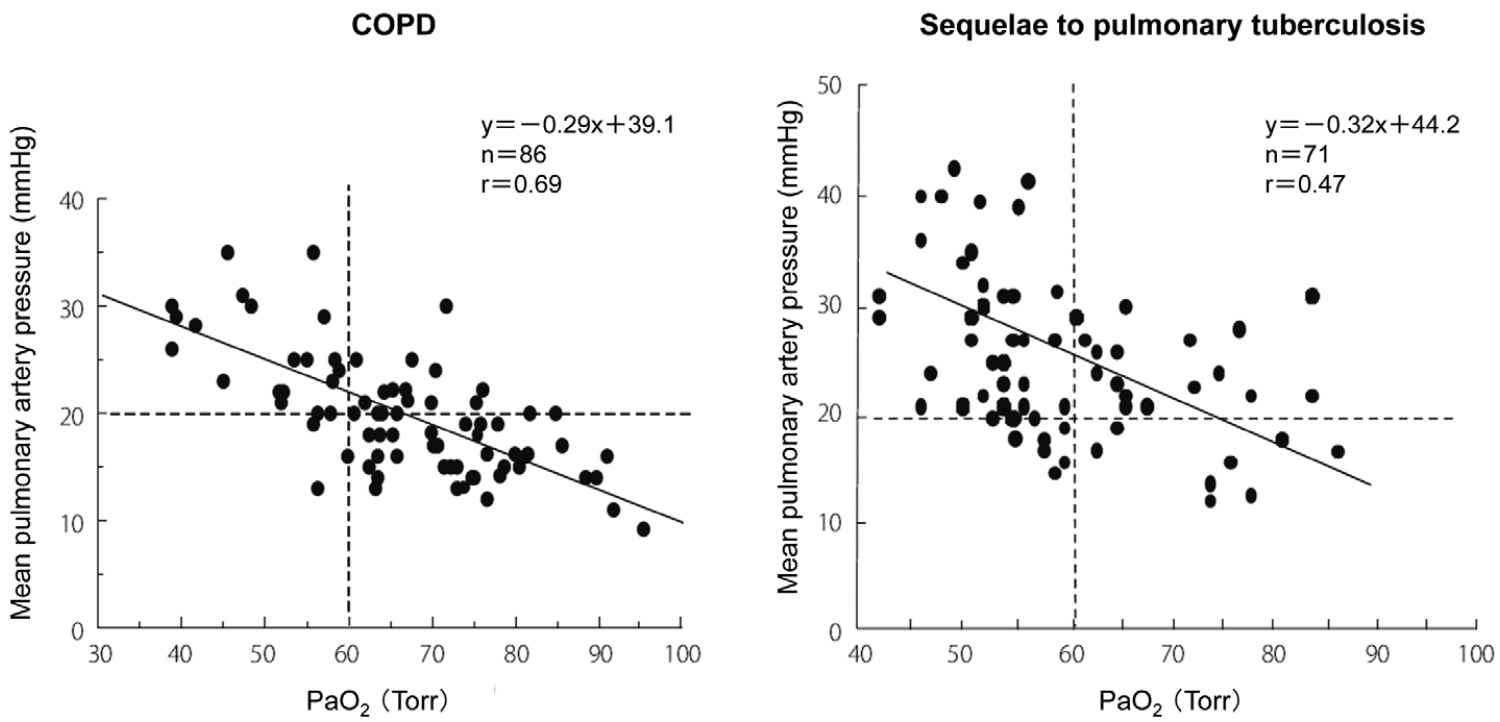

Figure 14. Correlation between $\mathrm{mPAP}$ and $\mathrm{PaO}_{2}$ in patients with $\mathrm{COPD}$ or sequelae to pulmonary tuberculosis. (Source: Tatsumi K, et al. 2005416)

with common features and have been playing an important role in dissemination of the concept of CPFE. However, from the viewpoint of implementing prospective interventional studies (which require clear-cut inclusion and exclusion criteria) and facilitating detailed pathophysiological analysis, there is no satisfactory criteria. Re-evaluation and review need to be conducted on the name and concept of CPFE from the viewpoint of how to achieve further pathophysiological clarification and improvement of treatment outcome and prognosis in patients with Group 3 pulmonary hypertension.

\subsection{Alveolar Hypoventilation Syndrome (AHS)}

\section{3.4.1 Epidemiology/Etiology}

Alveolar hypoventilation syndrome (AHS) presents with daytime alveolar hypoventilation (marked hypercapnia and hypoxia) despite no respiratory/thoracic/neurological/ muscular abnormality and no evident abnormality in the lung function tests. ${ }^{410}$ Abnormalities of chemical receptors constituting the chemical/metabolic regulation systems of respiration are estimated to be partially involved in its etiology, but details remain unknown. According to the classification proposed by the Respiratory Failure Study Group (Table 25), there are two phenotypes (A and B) of this disease. ${ }^{\mathbf{4 1 0}}$ Phenotype A was conventionally considered as primary AHS and has been found to involve gene PHOX2 mutation. ${ }^{410}$ The number of AHS patients in Japan is estimated to be about 40 for phenotype A alone and about 5,000 in total of phenotype A+B. Although the frequency of complication by pulmonary hypertension in AHS patients does not appear to be low, the actual frequency of complication is unknown because no data from a large number of cases is available.

\section{3.4.2 Pathophysiology/Prognosis}

Hypercapnia and hypoxia due to alveolar hypoventilation are major features of this disease, and these symptoms are aggravated during sleep. Pulmonary hypertension can develop as a result of hypoxic pulmonary vasoconstriction and vascular remodeling, but its mechanism is unknown. The prognosis of AHS is estimated to be usually poor when left untreated, ${ }^{\mathbf{2 1 3}}$ whereas the possibility of long-term survival has been reported for patients receiving appropriate treatment. ${ }^{411}$ Among the patients with pulmonary hypertension due to lung diseases, the group of pulmonary hypertension due to AHS or sleep dyspnea had better prognosis than the group of pulmonary hypertension due to COPD, interstitial pneumonia, or other lung diseases. ${ }^{\mathbf{4 1 2}}$

1 3.4.3 Diagnosis (Tables 25,26)

A diagnosis of AHS is made in cases in which arterial blood gas analysis reveals chronic and marked hypercapnia $\left(\mathrm{PaCO}_{2}>45\right.$ Torr) not attributable to any other disease and the symptoms for diagnostic criteria are present. ${ }^{410}$ Phenotype (A or B) is determined by polysomnography.

\subsubsection{Treatment}

No radical treatment is available for AHS, and symptomatic treatment is performed. For correction of alveolar hypoventilation, non-invasive positive pressure ventilation (NPPV) is used. In mild cases, home oxygen therapy (HOT) is sometimes used, but its efficacy has not been established. Effectiveness of diaphragmatic pacing has also been reported, ${ }^{\mathbf{4 1 3}}$ and there are case reports demonstrating its effect in alleviating pulmonary hypertension. ${ }^{\mathbf{4 1 4}, 415}$ In Japan oxygen therapy and NPPV are alternatives for treatment performed at present.

\subsection{Sequelae to Pulmonary Tuberculosis}

\section{Summary}

Sequelae to pulmonary tuberculosis are a syndrome involving sleep-disordered breathing, pulmonary circulatory disorder, and exertional dyspnea primarily caused by restrictive/obstructive ventilatory disorder. Pathophysiology 
and treatment of this condition resemble those of other lung diseases involving mixed restrictive and obstructive disorders.

\section{3.5.1 Epidemiology and Etiology}

Accurate epidemiological data on sequelae to pulmonary tuberculosis in Japan are unavailable. However, the percentage of patients with this condition among all patients receiving HOT has been decreasing.

The incidence of complication by pulmonary hypertension in patients with sequelae to pulmonary tuberculosis is considered to be high in patients with extensive lesions of atelectasis and cases of hypoxia after unilateral pneumonectomy or thoracoplasty. Possible mechanisms for onset of pulmonary hypertension include reduction or remodeling of pulmonary vascular bed following lung architecture destruction, and pulmonary vasoconstriction due to hypoxia.

\section{3.5.2 Diagnosis}

The diagnosis of sequelae to pulmonary tuberculosis is based on disease history and findings from chest diagnostic imaging. If pulmonary hypertension is suspected, echocardiography is first carried out, and a definite diagnosis is made on the basis of the results of RHC tests. In patients with this condition, estimation of mPAP from $\mathrm{PaO}_{2}$ is less easy than in patients with COPD. Even when hypoxia is absent, increased PAP is often seen (Figure 14). ${ }^{416}$

\section{3.5.3 Prognosis and Severity Rating}

Factors determining the prognosis of patients with sequelae to pulmonary tuberculosis are not known well. In many patients, the prognosis becomes poor following acute exacerbation of the condition due to increased respiratory failure or right-sided heart failure. Increased hypoxia or hypercapnia can also lead to aggravation of pulmonary hypertension. Severity rating requires right heart catheterization, but estimation of severity level is possible to some extent also from the arterial blood gas tension data.

\section{3.5.4 Treatment}

HOT and mechanical ventilation are alternatives for treatment of this condition. Medical treatment with bronchodilators and expectorants can improve pulmonary circulation by improvement of the airway condition.

\section{3.5.5 Responses to Treatment}

HOT can reduce mPAP in patients with pulmonary hypertension due to sequelae to pulmonary tuberculosis. ${ }^{417}$ Similar effects are expected also from mechanical ventilation therapy at home.

\section{3.5.6 Perspective and Open Issues for the Future}

The number of patients with sequelae to pulmonary tuberculosis will decrease further over time. New evidence concerning pulmonary hypertension due to sequelae to pulmonary tuberculosis is unlikely to be obtained, and pulmonary vasodilator therapy will not become indicated for patients with this condition. What is most important is to suppress new onset of severe pulmonary tuberculosis.

\subsection{Sleep-Disordered Breathing}

\section{3.6.1 Epidemiology/Etiology}

Complication by pulmonary hypertension is seen in about
$20 \%$ of patients with sleep-disordered breathing (SDB), and the complication rate is particularly high in patients presenting with severe nocturnal hypoxia. ${ }^{\mathbf{4 1 8}}$ Patients with severe SDB accompanied by obesity (body mass index (BMI) $\geq 30 \mathrm{~kg} / \mathrm{m}^{2}$ ), excessive drowsiness, and daytime hypercapnia are called "obesity hypoventilation syndrome (OHS)." In Japan, the number of patients with OHS is estimated at about $5,000 .{ }^{419} \mathrm{OHS}$ is accompanied by SDB, but complication by OHS in SDB cases is not frequent. ${ }^{\mathbf{2 2 0}}$ The incidence of complication by pulmonary hypertension in OHS cases is reported to be $58-88 \%$, higher than the incidence among cases of SDB. ${ }^{\mathbf{4 2 1 , 4 2 2}}$

\subsubsection{Pathophysiology/Prognosis}

If frequently repeating and severe transient hypoxia or OHS is seen in SDB patients, a possible mechanism is induction of pulmonary artery remodeling by pulmonary vasoconstriction due to persistent hypoxia. ${ }^{\mathbf{2 2 3}}$ Generally, the magnitude of PAP elevation in cases of pulmonary hypertension due to SDB is small, with MPAP not exceeding about $25-30 \mathrm{mmHg} .{ }^{423} \mathrm{~A}$ similar magnitude of PAP elevation has been reported also for cases of OHS, but the findings vary among different reports, including some reports of mPAP elevation to $50 \mathrm{mmHg} .{ }^{\mathbf{4 2 4}}$ Because it is known that left-sided heart failure is frequently complicated by SDB and that it can also be complicated by SDB+COPD, the presence of pulmonary hypertension due to left heart disease or COPD needs to be considered in SDB patients presenting with severe pulmonary hypertension. The prognosis of patients with OHS is poor compared to OHS-free patients of a similar obesity level ${ }^{425}$ In a cohort study of patients with pulmonary hypertension accompanied by lung disease and/or hypoxia, the three-year survival rate was $90 \%$ for patients with pulmonary hypertension accompanied by SDB or alveolar hypoventilation, thus showing prognosis poorer than patients with pulmonary hypertension accompanied by other lung disease and/or hypoxia. ${ }^{213}$

\subsubsection{Diagnosis}

Overnight pulse oximetry and simplified polysomnography are used for screening of SDB. In some severe cases, diagnosis and treatment can be readily performed or started on the basis of simplified polysomnography findings, but the diagnosis is established by polysomnography (performed inhospital) in many cases. ${ }^{\mathbf{4 2 6}}$ According to the OHS diagnostic criteria prepared by the Respiratory Failure Investigation/Study Group under the Specific Disease Study Program organized by the Ministry of Health and Welfare (renamed later), ${ }^{419}$ a diagnosis of OHS is made if all of the following requirements are satisfied: (1) grade 1 obesity (BMI $\geq 30 \mathrm{~kg} / \mathrm{m}^{2}$ ), (2) marked daytime drowsiness, (3) chronic hypercapnia ( $\mathrm{PaCO}_{2} \geq 45$ Torr), and (4) "severe" or higher severity level SDB. In OHS cases, echocardiography and respiratory function test are used to detect complication by left heart disease and COPD. Care needs to be taken of the fact that blood BNP level tends to be low in obese individuals.

\section{3.6.4 Treatment}

In patients with SDB or OHS showing an obese tendency, measures to reduce weight can be radical treatment, but it is difficult in many cases. Continuous positive airway pressure (CPAP) may be considered as the most effective method of treating SDB, although it is a symptomatic 


\begin{tabular}{|l|c|c|}
\hline \multicolumn{2}{|c|}{$\begin{array}{c}\text { Table 27. Recommendation and Evidence Level Concerning } \\
\text { Treatment for CTEPH }\end{array}$} \\
\hline \multicolumn{1}{|c|}{ Recommendation } & $\begin{array}{c}\text { Recommendation } \\
\text { Class }\end{array}$ & $\begin{array}{c}\text { Evidence } \\
\text { Level }\end{array}$ \\
\hline $\begin{array}{l}\text { Riociguat for CTEPH ineligible for } \\
\text { PEA or remaining/recurring PH } \\
\text { after PEA }\end{array}$ & $\mathrm{I}$ & $\mathrm{B}$ \\
\hline Anticoagulation for CTEPH & $\mathrm{I}$ & $\mathrm{C}$ \\
\hline $\begin{array}{l}\text { PEA under deep hypothermic } \\
\text { circulatory arrest for central type } \\
\text { CTEPH }\end{array}$ & $\mathrm{I}$ & $\mathrm{C}$ \\
\hline BPA for CTEPH ineligible for PEA & $\mathrm{I}$ & $\mathrm{C}$ \\
\hline $\begin{array}{l}\text { Oxygen therapy for CTEPH } \\
\text { presenting with hypoxia }\end{array}$ & $\mathrm{Ila}$ & $\mathrm{C}$ \\
\hline $\begin{array}{l}\text { PEA under deep hypothermic } \\
\text { circulatory arrest for peripheral } \\
\text { type CTEPH }\end{array}$ & $\mathrm{lla}$ & $\mathrm{C}$ \\
\hline $\begin{array}{l}\text { Treatment with vasodilators other } \\
\text { than riociguat for CTEPH ineligi- } \\
\text { ble for PEA or remaining/recur- } \\
\text { ring PH after PEA }\end{array}$ & $\mathrm{llb}$ & $\mathrm{B}$ \\
\hline $\begin{array}{l}\text { Diuretic/cardiotonic therapy for } \\
\text { CTEPH accompanied by right- } \\
\text { sided heart failure }\end{array}$ & $\mathrm{llb}$ & $\mathrm{C}$ \\
\hline Lung transplantation for CTEPH & $\mathrm{llb}$ & $\mathrm{C}$ \\
\hline
\end{tabular}

therapy. Several reports, including small-scale randomized controlled trials, demonstrated reduction of PAP following several months of CPAP therapy, ${ }^{427,428}$ Before CPAP was adopted extensively, efficacy of surgical treatment such as tracheotomy was reported, including its effects in reducing PAP and improving the right ventricular ejection fraction. ${ }^{429-431}$ However, application of these operative procedures for treatment of SDB has been quite rare in recent years, regardless of the presence/absence of complication by pulmonary hypertension in the patients requiring treatment of SDB. Now, surgical treatment should be considered only when it is needed (e.g., in cases in which CPAP is difficult to perform). Long-term oxygen therapy is sometimes considered in patients in whom CPAP is difficult to perform, but its efficacy is not sufficient when performed separately and its effect on PAP is not evident. Specific drug therapy for pulmonary hypertension is also considered in cases in which complication by left heart disease and COPD has been ruled out and pulmonary hypertension persists even after appropriate treatment of SDB. However, when performing such drug therapy, it needs to be taken into consideration that no evaluation of such drug therapy in patients with pulmonary hypertension due to SDB has been reported. Also in cases of OHS, the nocturnal SDB can be sometimes treated with CPAP. However, in view of the report that 3-month NPPV in patients with OHS reduced PAP, ${ }^{424}$ it is important to maintain ventilation by NPPV if nocturnal hypoventilation is evident despite treatment with CPAP.

\section{Chronic Thromboembolic Pulmonary Hypertension (CTEPH)}

\section{Summary}

Chronic thromboembolic pulmonary hypertension (CTEPH) is a rare complication of acute pulmonary embolism. The thrombi remaining after incomplete thrombolysis become organized thrombi and cause stenoses or obstructions of the pulmonary artery, leading to pulmonary hypertension by increased pulmonary vascular resistance. Therefore, CTEPH can be resolved by complete removal of the organized thrombi from the pulmonary artery. Unlike the other types of pulmonary hypertension, standard treatment for CTEPH is surgical treatment, in cases of CTEPH ineligible for surgery, medical treatment with pulmonary vasodilators has been performed. Recently, balloon pulmonary angioplasty (BPA) has begun to be performed primarily in Japan, yielding outcomes comparable to those of surgical treatment. Recommendations related to CTEPH treatment are summarized in Table 27.

\section{I.1 Epidemiology/Etiology}

\section{a. Epidemiology}

According to the results of investigation by the "Respiratory Failure Investigation/Study" Group under the Intractable Disease Policy Study Program, 2,829 individuals across the country possessed a certificate for beneficiary of specific public financial subsidy to care for CTEPH (an officially listed intractable disease) at the end of 2015 (source: same as Document 2).

\section{b. Etiology}

The incidence of pulmonary thromboembolism (PTE), including acute and chronic types, in Japan is estimated to be lower than that in Western countries. Also in a study based on the data from postmortem pathological examination (Annual of the Pathological Autopsy Cases in Japan), the incidence of PTE was described to be about $1 / 10$ of that in the United States. In the United States, acute PTE is estimated to develop in 500,000-600,000 individuals per year, and about $0.1-0.5 \%$ of patients surviving the acute stage of PTE reach the stage of CTEPH. A subsequent report, however, demonstrated a chronic course followed by $3.8 \%$ of all acute cases. It is therefore important to consider that acute PTE can lead to CTEPH.

The severity of vascular obstruction is important as a factor associated with pulmonary hypertension. Many cases have $40 \%$ or more pulmonary vascular obstruction, but the correlation between the obstruction rate and the pulmonary vascular resistance (PVR) is not high. Involvement of repeated thrombosis and increased thrombus formation in the pulmonary arteries also may be possible. Furthermore, the involvement of small vessel disease has been suggested, including: (1) thrombotic obstruction of elastic arteries at the subsegmental level as seen in cases of PAH, (2) vascular lesions of muscular arteries associated with increased blood flow through thrombus-free sites, and (3) vascular lesions of muscular arteries associated with anastomosis to bronchial arteries distant from the thrombusobstructed site. In Japan, CTEPH is seen more frequently in females than in males, and there is a report concerning the presence of a disease type associated with human leukocyte antigen (HLA)-B*5201 and HLA-DPB1*0202 (factors known to be associated with low incidence of deep vein thrombosis). Because these types of HLA do not correlate with acute pulmonary thromboembolism and their frequency is quite low in Western countries, this finding suggests that in Japan there are cases of CTEPH whose onset mechanism differs from that in cases in Western countries. 


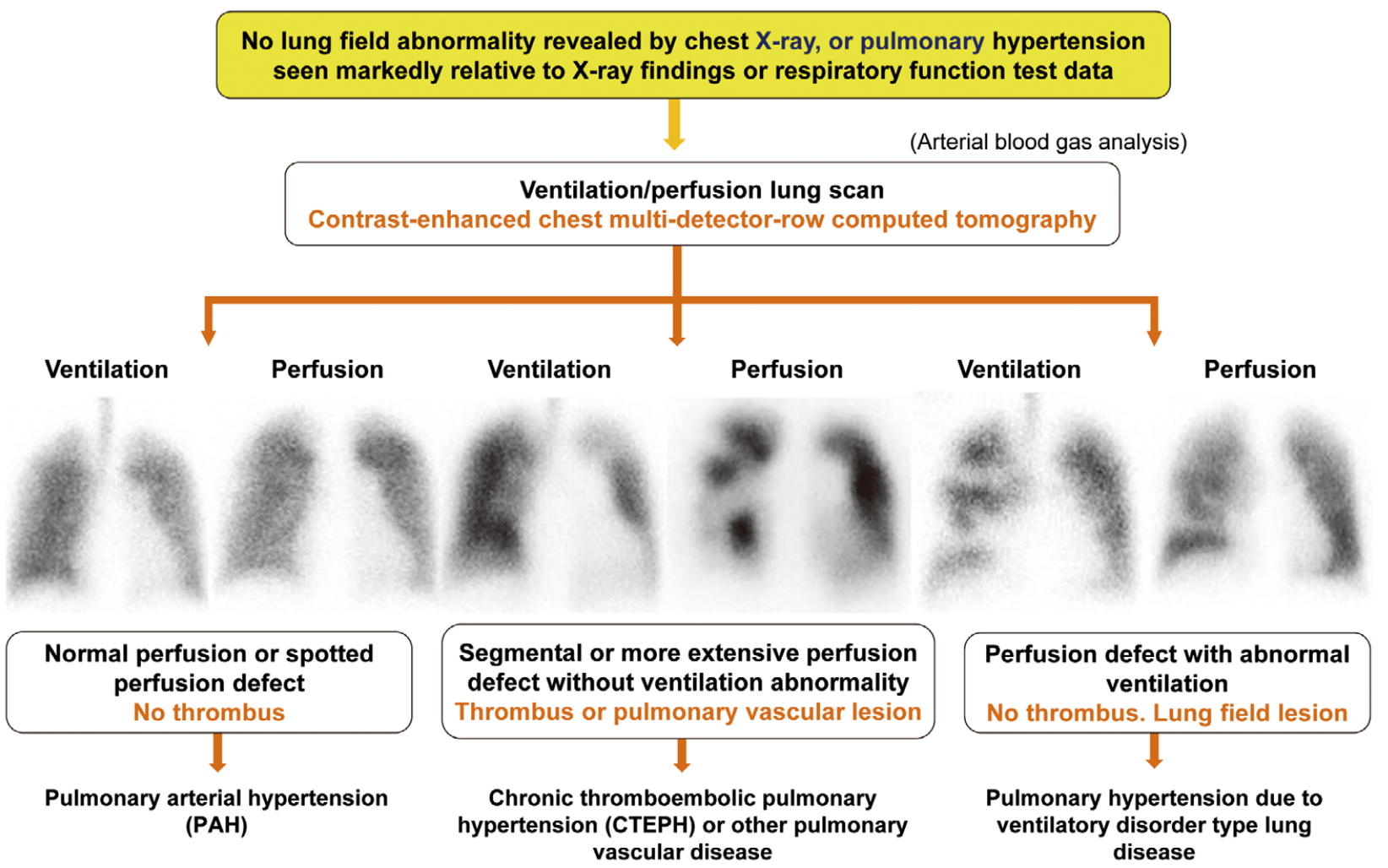

Figure 15. Approaches to unexplained pulmonary hypertension and positioning of CTEPH.

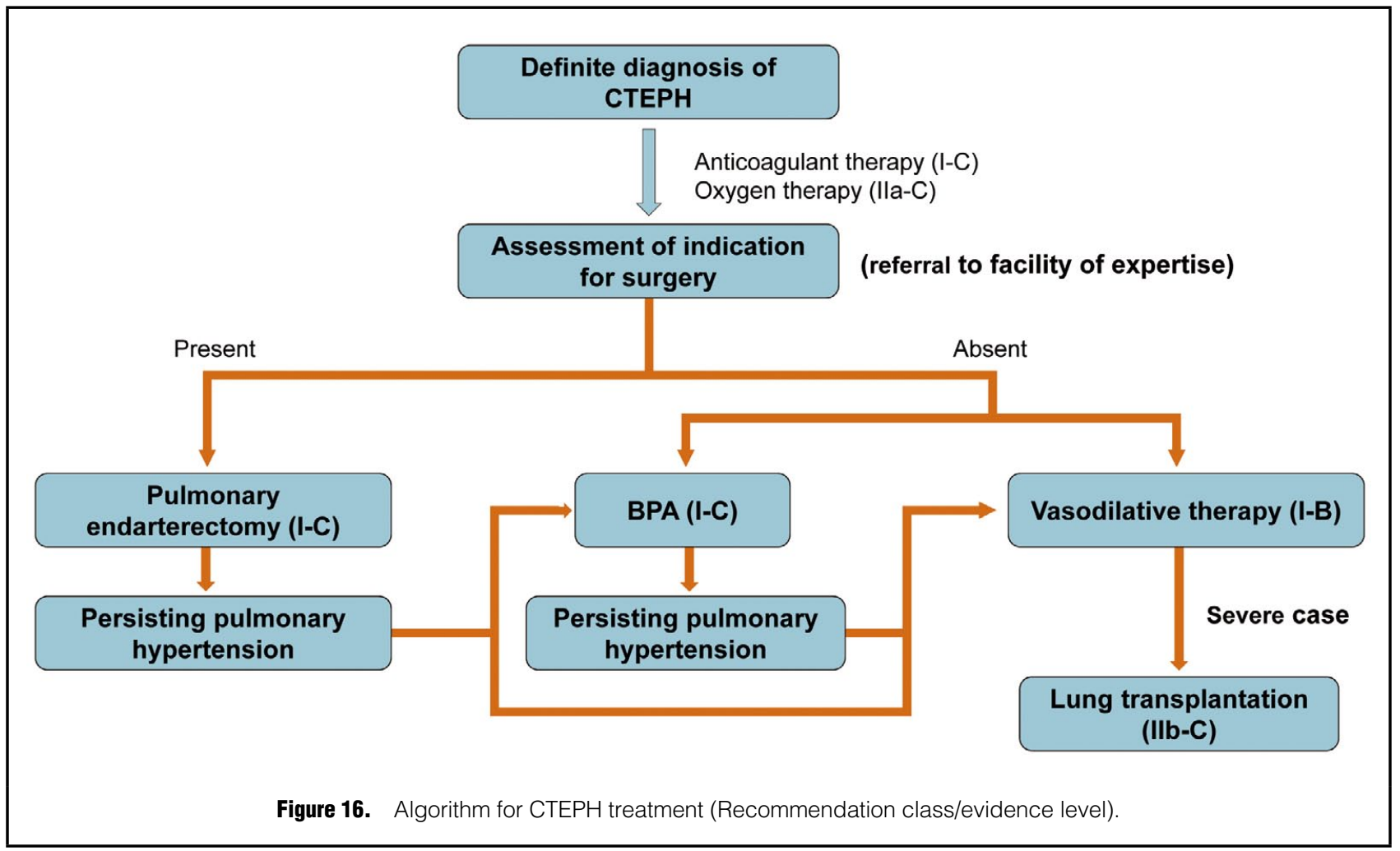



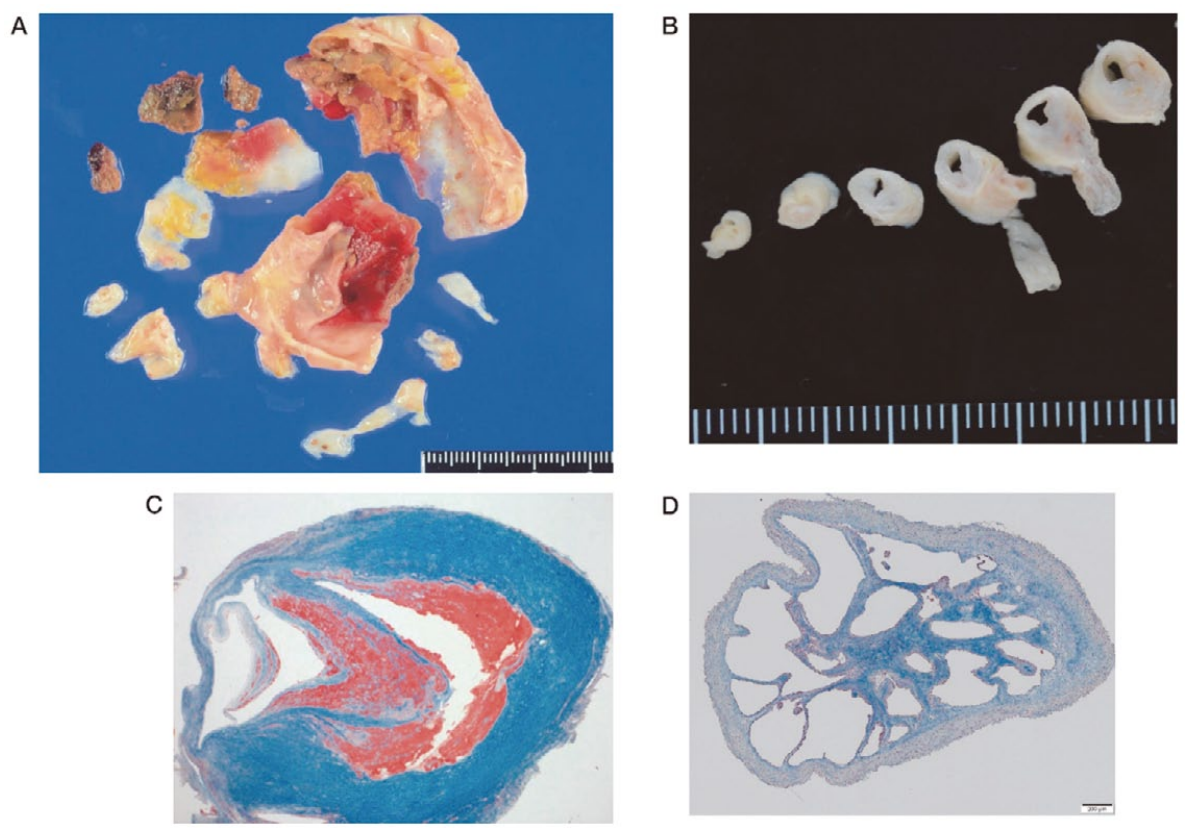

Figure 17. Surgically resected intima of pulmonary artery from a patient with CTEPH. (A) Massive organizing thrombi adherent in the main pulmonary artery. (B) The peripheral pulmonary artery is often re-open. (C) Severe stenosis by lamellar thrombus is noted in the pulmonary lobar artery. (D) Cribriform signs of recanalization is seen at the peripheral segmental artery.

\section{4.2 Symptoms/Clinical Findings}

Major symptoms and clinical findings include: (1) shortness of breath on exertion, (2) clinical symptoms of acute cases (sudden dyspnea, chest pain, syncope) having occurred at least once, (3) clinical symptoms of deep vein thrombosis (DVT) of legs (leg swelling and pain) having occurred at least once, (4) pulmonary vascular murmur heard in the lung field, and (5) abnormalities suggesting pulmonary hypertension (e.g., enhanced pulmonary artery component of the second heart sound) revealed by chest auscultation.

\section{4.3 Diagnosis and Treatment of CTEPH}

Two steps are needed for the diagnosis of CTEPH: (1) diagnosis of pulmonary hypertension on the basis of right heart catheterization, and (2) ruling out the other diseases that can cause PH. The approaches to cases of unexplained pulmonary hypertension and the positioning of CTEPH are illustrated in Figure 15.

The criteria for diagnosis of CTEPH and the criteria for official registration of CTEPH patients under the intractable disease registration program are given in "IV. Pulmonary hypertension under the measures against intractable diseases 'officially listed intractable disease, Document 2. Chronic thromboembolic pulmonary hypertension (CTEPH) (Listed Intractable Disease No. 88)" of these guidelines.

The algorithm for treatment of CTEPH is shown in Figure 16. For a definite diagnosis, it is necessary to demonstrate that the condition does not improve despite anticoagulant therapy for a sufficiently long period of time. Also, after a definite diagnosis, anticoagulant therapy needs to be continued for preventing recurrence of thrombosis and secondary thrombus formation. In cases contraindicated against anticoagulant therapy or patients with acute pulmonary thromboembolism recurring during anticoagulant therapy, insertion of an inferior vena cava (IVC) filter should be considered. In addition, as needed, treatment of hypoxia and right-sided heart failure should be additionally performed. Treatment of pulmonary vascular lesions is first aimed at resolving pulmonary hypertension to prevent right-sided heart failure and to improve the prognosis of patients. Second, it is aimed at correcting ventilationperfusion imbalance to improve oxygenation and to alleviate symptoms such as shortness of breath. At present, medical treatment (pulmonary vasodilator therapy), surgical treatment, and BPA are performed as alternatives for treatment in Japan. Of these treatment methods, only surgical pulmonary endarterectomy (PEA) can achieve the two purposes mentioned above, i.e., the only treatment method recognized as to the potential of radical treatment. ${ }^{\mathbf{3 2} 2}$ Vasodilator therapy can improve hemodynamics to some extent ${ }^{433}$ but is unlikely to improve oxygenation. Although no direct comparison has been made, BPA performed at experienced facilities improved hemodynamics more markedly than pulmonary vasodilator therapy. ${ }^{\mathbf{4 3 4} 436}$ However, it is not easy to achieve improvement of oxygenation by BPA. It may be, therefore, reasonable that the indications for PEA are first assessed and, in cases not indicated for PEA or pulmonary hypertension remaining after PEA, application of BPA is considered. In cases not indicated for BPA, pulmonary vasodilator therapy should be performed and, if the condition remains severe despite this therapy, lung transplantation should be considered.

\section{| 4.4 Pathology 437,438}

After the Dana Point Classification (2009), ${ }^{7}$ the name "CTEPH" was widely accepted. CTEPH is not attributable 
A

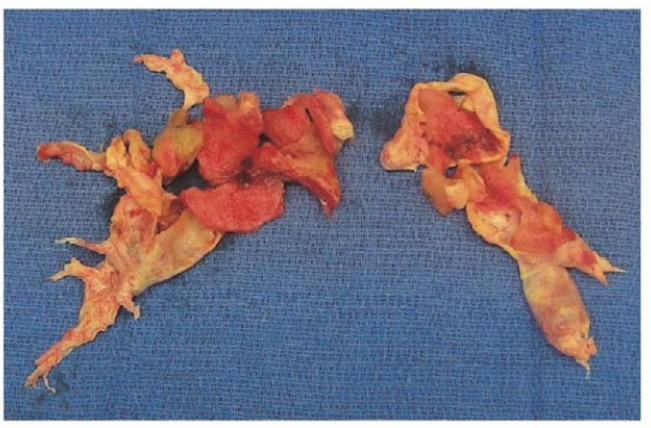

B

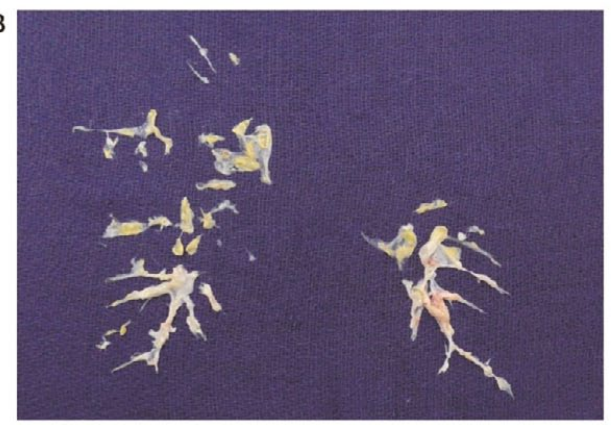

Figure 18. Pulmonary artery tunica intima resected surgically from a patient with CTEPH. (A) Pulmonary artery tunica intima of a patient with central type CTEPH. (B) Pulmonary artery tunica intima of a patient with peripheral type CTEPH.

to the pulmonary artery itself, which is the direct cause of pulmonary arterial hypertension (PAH). Instead, in the presence of underlying disease, such as (1) DVT and (2) coagulation abnormalities (e.g., antiphospholipid antibody positive and protein $\mathrm{C} /$ protein $\mathrm{S}$ deficiency), thromboembolism to pulmonary artery may develop repeatedly and, with the increase in the number of obstructed vessels, eventually resulting in pulmonary hypertension. Many of the pulmonary artery lesions involved in CTEPH have been formed in the following manner. That is, the thrombi transported repeatedly from the deep veins cause obstruction of the pulmonary lobar artery as well as pulmonary segmental and subsegmental arteries, leading to organization of the thromboembolism at these sites. These lesions can be divided into the central type (intimal thickening from the main pulmonary artery to the pulmonary segmental arteries) and the peripheral type (intimal thickening from the segmental artery to distal artery). There are also cases of mixed central and peripheral type characterized by diffuse obstruction of lumen. Although rarely, there are also cases in which thrombus formation is seen only in the peripheral pulmonary artery, clinically mimicking idiopathic PAH. Signs of organized thromboembolism and peripheral recanalization were noted in the obstructed part of the pulmonary artery resected by PEA $^{439}$ (Figure 17A to $\mathbf{D})$. Also, the non-obstructed segment of the pulmonary artery occasionally shows diffuse intimal thickening as a secondary change associated with pulmonary hypertension. The dilated pulmonary artery trunk assumes an aneurysmlike form, often leading to parietal thrombus formation. Old pulmonary infarction is sometimes detected in the obstructed area, and obstruction of pulmonary veins can also occur. When obstruction affects only the peripheral branches, distal approach by PEA will be difficult.

\section{| 4.5 Surgical Treatment}

Surgical treatment of CTEPH began to be reported overseas in the 1960 s. In Japan, it was first reported in 1986 by Nakajima et al. who attempted an approach with lateral thoracotomy without using extracorporeal circulation. ${ }^{\mathbf{4 4 0}}$ The current technique of PEA for CTEPH was developed in the 1970 s at University of California San Diego (UCSD) and it is aimed at simultaneous resection of thrombi in bilateral pulmonary arteries and hypertrophic tunica intima under extracorporeal circulation (ECC) and deep

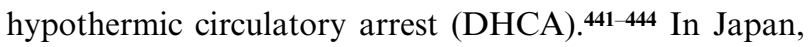
this technique was clinically introduced in the $1990 \mathrm{~s}^{\mathbf{4 4 5} 447}$ and has been the only method of radical treatment for CTEPH since then. According to the annual report by the Japanese Association for Thoracic Surgery, this operative procedure has been performed to about 50 cases annually (61 cases in 2014), with the in-hospital mortality rate being $9.8 \% .{ }^{448}$ Most cases of chronic pulmonary artery thromboembolism (particularly cases in which surgical treatment is considered) are accompanied by pulmonary hypertension. This section will describe the indications for PEA, its technique, and outcome in patients with CTEPH.

\subsubsection{Pulmonary Endarterectomy (PEA) \\ a. Indications for PEA \\ i. Criteria for Judgment of Indications}

(1) Conventional UCSD criteria for PEA indications ${ }^{443}$ Whether a given patient is indicated for PEA is determined on the basis of symptoms, echocardiography, contrastenhanced chest CT scan, and ventilation/perfusion lung scan as well as pulmonary arteriography (PAG) and right heart catheterization.

- Mean pulmonary artery pressure (mPAP) $\geq 30 \mathrm{mmHg}$, PVR $\geq 300$ dyne $\cdot \mathrm{sec} \cdot \mathrm{cm}^{-5}$

- NYHA/WHO functional class $\geq I I I$

- Proximal end of pulmonary artery lesion located at a surgically accessible site

- Without severe complication (accompany disease)

(2) Latest ESC criteria for PEA indications (2015) ${ }^{1}$

The following criteria have been added. The scope of indications for PEA has been gradually expanded following improvement in the outcome of this operation.

- NYHA/WHO functional class II is also indicated in addition to the class $\geq$ IIII.

- Peripheral lesions at the segmental pulmonary artery level are also indicated if surgically accessible.

- Advanced age, high PVR, and right ventricular dysfunction do not serve as factors denying indications for PEA.

\section{ii. Localization of Pulmonary Artery Lesions (Morphology)}

CTEPH can be morphologically divided into the following two types.

- Central type: Lesions located in the pulmonary artery main trunk to the lobar/segmental arteries (Figure 18A)

- Peripheral type: Primarily lesions of small arteries peripheral to the segmental artery (Figure 18B) 
UCSD proposed a more detailed classification based on PEA specimens, as shown below. 449

- Type I: Fresh thrombus noted in the main pulmonary artery or interlobar artery

- Type II: Hypertrophic tunica intima or fibrous tissue noted on the central side of segmental artery regardless of the presence/absence of organized thrombus

- Type III: Hypertrophic tunica intima or fibrous tissue noted in distal segmental artery alone to subsegmental artery regardless of the presence/absence of organized thrombus

- Type IV: Arteriolar lesions noted in the more peripheral region without macroscopic thromboembolic lesions

Types I and II correspond to the central type and are well indicated for PEA. Types III and IV are the peripheral type and are not always indicated for PEA.1,432,444,449,450 More than $80 \%$ of the cases managed at UCSD were the central type (Type I 37.4\%, II 49.0\%, III 12.0\%, IV 1.6\%). ${ }^{432}$ In contrast, Japanese patients with CTEPH often have peripheral lesions and less frequently have central lesions and are often obscure as to the history of PTE, making it difficult to perform PEA. ${ }^{450}$ Application of PEA to cases of peripheral lesions was recently evaluated at UCSD, reporting that the percentage of type III cases among the first 1,000 cases of the 1,500 cases managed during the period 1999 2001 was $13.1 \%$ and the percentage rose to $21.4 \%$ among the subsequent 500 cases, without showing aggravation of the outcome of treatment from the first to latter group. On the basis of these results, application of PEA to the peripheral type was recommended. ${ }^{432}$

More recently, a new classification of surgery (level) was published. ${ }^{451}$ This classification is intended to divide the cases according to the difficulty level for application of PEA depending on the location of major lesions, regardless of the presence/absence of fresh thrombus.

- Level 0: Without CTEPH lesions

- Level I: Lesions begin in bilateral main pulmonary artery (Level IC: Accompanied by unilateral complete obstruction of main pulmonary artery, C; abbreviation of complete)

- Level II: Lesions begin in lobar artery (periphery of upper lobar bifurcation)

- Level III: Lesions begin in segmental artery

- Level IV: Lesions present in subsegmental artery

\section{iii. Pulmonary Hypertension, Right-Sided Cardiac Function, Others}

In the past, PVR $\geq 1,100 \mathrm{dyne} \cdot \mathrm{sec} \cdot \mathrm{cm}^{-5}$ and mPAP $\geq 50 \mathrm{mmHg}$ were listed as risk factors for perioperative death. ${ }^{452,453}$ Recently, there is no level of mPAP or PVR set as the upper limit for indications of PEA. ${ }^{1}$ Although dilatation and hypertrophy are seen in the right ventricle and its wall motion has been reduced in patients with this disease, their abnormality is expected to become less severe if pulmonary hypertension is alleviated by PEA, accompanied by improvement in wall motions. ${ }^{454} \mathrm{As}$ a result, the tricuspid valve junction is restored and tricuspid insufficiency is alleviated. ${ }^{455,456}$ Right ventricular dysfunction is not a factor denying indications for PEA. ${ }^{1}$ In elderly patients, cardiopulmonary function tends to be compromised, accompanied by reduction in respiratory function and the presence of other disease. For this reason, advanced age can be a risk factor for early death after PEA. ${ }^{450,457}$ However, extreme aggravation of the outcome of PEA in elderly patients has not been recently reported, and the ESC/ERS Guidelines for the Diagnosis and Treatment of Pulmonary Hypertension 2015 also acknowledge applica- tion of PEA to elderly patients. ${ }^{1}$ The outcome of PEA is relatively favorable in re-operated cases ${ }^{458}$ pediatric cases, ${ }^{459}$ and cases having undergone simultaneous surgery for other heart disease. ${ }^{460}$

\section{b. Operative Procedure PEA}

i. Preoperative Steps

- Anticoagulant therapy: Warfarin is switched to serial drip infusion of heparin several days before PEA.

- IVC filter: Although this was routinely used before, its use has recently discontinued unless complication by severe DVT is present.

- Preoperative drug therapy: In cases of severe pulmonary hypertension, alleviation of pulmonary hypertension is attempted by serial drip infusion of prostacyclin $\left(\mathrm{PGI}_{2}\right)^{461}$ or other drug therapy. ${ }^{462}$

\section{ii. Anesthesia}

In severe cases, care needs to be taken of hypotension when anesthesia is introduced.

- To treat pulmonary hemorrhage, the airway is intubated with a double-lumen tracheal tube.

- A central venous line and a Swan-Ganz catheter are inserted.

\section{iii PEA procedure 443,444}

- Approach: An approach is performed under median sternal incision.

- ECC establishment/systemic cooling: After administration of heparin, ECC is establishment by supplying blood via the ascending aorta and guiding out blood from the superior vena cava (SVC) and inferior vena cava (IVC). After insertion of left atrium or left ventricle vent and a pulmonary artery vent, the whole body is cooled to reduce the nasopharyngeal/tympanic membrane temperature to $18^{\circ} \mathrm{C}$. The head is then cooled locally.

- Right atrium incision: Atrium septal defect and patent foramen ovale, if any, are closed. The state of tricuspid valve is also examined.

- Right-sided PEA: Deciding the plane to be freed is most important. If freeing is done too deeply, it can injure the tunica externa, possibly leading to fatal lung/airway bleeding. A retractor is placed between the SVC and the ascending aorta, and the middle of the anterior plane of the right pulmonary artery is vertically incised. Any thrombus found is removed, to find the layer to be freed. The ascending aorta is occluded and the myocardial protective fluid is infused. During DHCA $\left(18^{\circ} \mathrm{C}\right)$, PEA is carried out toward the segmental/subsegmental artery using a Jamieson dissector. Cycles of 15-minute DHCA and 10-minute systemic reperfusion are repeated. The pulmonary artery is closed by double suturing.

- Left-sided PEA: The left PA is vertically incised from the pulmonary artery trunk to the pericardium-everted part. PEA is performed in a manner similar to the right side. Warming to restore the body temperature is started, and the left pulmonary artery is subjected to double closure.

- Additional surgery: If any other heart surgery is needed, it is performed during the body temperature restoring procedure.

- Weaning from ECC: Before pulmonary perfusion is resumed, the positive end-expiratory pressure (PEEP) $10 \mathrm{cmH}_{2} \mathrm{O}$ is started to prevent reperfusion injury of lungs. Upon completion of body temperature restoration, the patient is carefully weaned from ECC. 
- Others: In cases of severe residual pulmonary hypertension or airway bleeding, percutaneous cardiopulmonary support (PCPS)/extracorporeal membrane oxygenation (ECMO) is given. If an intra aortic balloon pumping (IABP) is simultaneously inserted, the pulsatile flow can be maintained and hemodynamics can be improved.

\section{iv. Intraoperative/Postoperative Management}

- Circulation management: If the right-side heart function is compromised, dopamine and dobutamine are simultaneously used. Usually, norepinephrine is used at a relatively high dose level $(0.1-0.5 \gamma)$ with a goal set at maintaining systemic blood pressure $\geq 80 \mathrm{mmHg}$. Cardiac index is often about $2 \mathrm{~L} / \mathrm{min} / \mathrm{m}^{2}$, but this is acceptable if urine volume is preserved. Residual pulmonary hypertension is treated by inhalation of nitrogen monoxide (NO).

- Respiratory control: After admission to ICU, the patient is managed with mechanical ventilation (PEEP $10 \mathrm{cmH}_{2} \mathrm{O}$ ) under sedation. During this period, diuresis needs to be performed and the influence from lung reperfusion injury, ECC, and DHCA are removed. If done, pulmonary hypertension will gradually alleviate, usually allowing weaning from intubation within 24 hours.

- Anticoagulant therapy: Serial drip infusion of unfractionated heparin is started on the first postoperative day and it is later switched to oral warfarin treatment.

- Postoperative rehabilitation: After being discharged from the ICU, the patient is gradually guided to leave the bed in the general ward and to receive rehabilitation for about 2 weeks before being discharged from hospital. Weaning from oxygen therapy is done slowly.

\section{c. PEA Outcome \\ i. Early Results}

The outcome of PEA has been improving due to establishment of the criteria for indications, improvement in operative procedure/perioperative management, and accumulation of experience. At the UCSD, the mortality rate among the 500 cases recently managed was quite favorable $(2.2 \%), 432$ and the rate was about $5 \%$ in the international registry study and the data at other facilities $\mathbf{4 6 3 , 4 6 4}$ and $9.8 \%$ according to the report by the Japanese Association for Thoracic Surgery. ${ }^{448}$ Risk factors for early death after PEA reported from overseas include prolonged ECC and increased PVR, ${ }^{443}$ preoperative $\mathrm{mPAP}>50 \mathrm{mmHg}$ and PVR $>1,100$ dyne $\cdot \mathrm{sec} \cdot \mathrm{cm}^{-5}$, 452 advanced age, right atrial pressure, NYHA, number of affected pulmonary artery branches, and preoperative hypoxia. ${ }^{457,465}$ Risk factors for early death reported in Japan include advanced age $(\geq 60$ years), surgery before $2000,{ }^{450}$ and PVR $\geq 1,052$ dyne $\cdot \mathrm{sec} \cdot$ $\mathrm{cm}^{-5}{ }^{466}$ Postoperative residual pulmonary hypertension (PVR $\geq 400$ dyne $\cdot \sec \cdot \mathrm{cm}^{-5}$ ) is seen in $16.0-33.7 \%$ of all cases, ${ }^{450,457,467}$ with its risk factors being advanced age, right atrial pressure, sex (female), large number of nonrecanalized branches, and peripheral lesions.

\section{ii. Long-Term Results}

Long-term survival rates have been favorable. UCSD reported the survival rate among 1,410 patients to be $82 \%$ at 5 years and $75 \%$ at 10 years, ${ }^{432}$ and the Papword Hospital (UK) reported the survival rate among 229 patients to be $94 \%$ at 3 years, $92.5 \%$ at 5 years, and $88.3 \%$ at 10 years. ${ }^{467}$ In Japan, the National Cerebral and Cardiovascular Center reported the survival rate among 130 patients to be $95.2 \%$ at 5 years and $93.3 \%$ at 7 years, and Chiba University reported the rate among 77 patients to be $84 \%$ at 5 years and $82 \%$ at 10 years. ${ }^{468}$ Risk factors for death after the acute postoperative period include residual pulmonary hypertension and postoperative NYHA/WHO functional class III/IV. Furthermore, recurrence of CTEPH was noted in $2.5-4.6 \%$ of all cases. ${ }^{463,467}$

\subsubsection{Lung Transplantation}

Another operative procedure is lung or cardiopulmonary transplantation. However, the long-term outcome of lung or cardiopulmonary transplantation is inferior to the long-term outcome of PEA, and the indications of lung transplantation are limited by problems related to immunosuppressors and shortage of donors. ${ }^{1}$ Among all CTEPH patients, this procedure is indicated in part of the cases morphologically rated as the peripheral type and difficult for PEA or BPA, or cases after failure in PEA or having recurred after PEA. In Japan, shortage of brain-dead donors is limiting the indications of lung or cardiopulmonary transplantation.

\subsection{Drug Therapy and Oxygen Therapy I 4.6.1 Pulmonary Vasodilators}

CTEPH is a disease in which the organized thrombus physically reduces blood flow in the pulmonary artery. It is difficult to improve blood flow in the major lesions (the sites of organized thrombus) effectively using pulmonary vasodilators. Furthermore, since the distribution and severity of organized thrombus vary among individual cases, administration of pulmonary vasodilators occasionally increases the pulmonary ventilation-perfusion imbalance, resulting in aggravation of oxygenation. Pulmonary vasodilators are considered to be indicated in cases of non-operable CTEPH rated as NYHA/WHO functional class II or higher, cases of CTEPH at increased risk for PEA and cases of post-PEA with residual or recurrent pulmonary hypertension.

In the past, classical vasodilator therapy was attempted in cases of CTEPH using calcium antagonists, nitrides, and angiotensin-converting enzyme (ACE) inhibitors, but no evidence supporting the effectiveness of these drugs has been obtained. CTEPH and PAH were originally defined as two different diseases. In recent years, however, proposal was made about a new view about the pathophysiology of CTEPH, according to which CTEPH is considered a small vessel disease or microvascular disease of the pulmonary artery and is partially overlapped with $\mathrm{PAH} .{ }^{469}$ This view reflects the idea that acute PTE is only a factor that is a trigger during the course of CTEPH and that in part of all CTEPH cases the pulmonary vessels undergo remodeling at sites other than the area in which an organized thrombus has been formed by some mechanism, resulting in increased vascular obstruction and leading to peripheral type CTEPH (as a rationale for this mechanism, the presence of CTEPH patients with peripheral vascular lesions similar to those of PAH was cited ${ }^{469}$ ). For this reason, treatment with pulmonary vasodilators in a manner similar to that for $\mathrm{PAH}$ patients may be effective also in cases of CTEPH with features of small vessel disease.

For a long period of time, there was no pulmonary vasodilator whose use in patients with CTEPH was covered by health insurance. Recently, a soluble cyclase guanylate stimulator (riociguat) was covered by health insurance for the first time in the world after clinical trials (in Japan, it 
was covered in January 2014 by health insurance with the indications of "chronic thromboembolic pulmonary hypertension not indicated for surgical treatment or remaining/ recurring after surgical treatment”). In CHEST-1 Study, $\mathbf{4 3 3}$ designed as a placebo-controlled randomized study involving 261 non-operative CTEPH (including cases of post-PEA residual pulmonary hypertension), the 6-minute walk distance (a primary endpoint) increased significantly to $39 \mathrm{~m}$ during the 16-week observation period in the riociguat group, accompanied by significant improvement of PVR to $246 \mathrm{dyne} \cdot \mathrm{sec} \cdot \mathrm{cm}^{-5}$. Other than these parameters, $\mathrm{N}$-terminal pro-brain natriuretic peptide (NT-proBNP) level, NYHA/WHO functional class, and cardiac output also improved significantly in the riociguat group. In CHEST-2 Study (an open-label extension study after CHEST-1 Study), ${ }^{470}$ riociguat was administered to all 237 of the subjects, resulting in a further increase in the 6-minute walk distance during the one-year observation period. The survival rate was $97 \%$ at one year. Following these results, now riociguat is used as the first-choice pulmonary vasodilator for patients with CTEPH.

In addition, clinical studies have been conducted also on

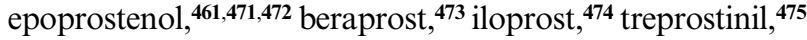
sildenafil, ${ }^{476}$ and bosentan, ${ }^{477}$ in open-label studies and placebo-controlled studies of sildenafil demonstrated significant improvement in hemodynamics, 6-minute walk distance, and BNP level. However, because none of these drugs has been covered by health insurance in Japan, their use is obliged to assume the form of "off-label use." At present, domestic clinical studies of $\mathrm{PGI}_{2}$ receptor agonists and an overseas clinical trial of macitentan (MERIT-1 Study) are under way. Publication of their results is being awaited.

Attempts have also been made to improve the outcome of surgery by treatment with pulmonary vasodilators preoperatively (as a bridge to PEA) in severe operable cases of CTEPH, but sufficient evidence has not yet been obtained. ${ }^{\mathbf{4 6 2}, \mathbf{4 7 8}}$

\section{4.6.2 Thrombolytic Therapy}

There is no evidence supporting the effectiveness of thrombolytic therapy for organized thrombus in CTEPH patients. However, in cases in which the condition has acutely exacerbated during the course of management, a condition called "chronic PTE complicated by acute PTE (acute on chronic PTE)" needs to be considered. If the levels of clotting/fibrinolysis system molecular markers, such as D-dimer, is increased, alleviation may be achieved by thrombolytic therapy. Thus, thrombolytic therapy should be performed in cases in which the involvement of acute on chronic PTE is suggested by data/findings (including the findings from diagnostic imaging).

\section{4.6.3 Anticoagulant Therapy}

The prognosis of untreated CTEPH gets poorer as the hemodynamic disorder increases, and the presence of cases showing aggravation of hemodynamics with time has been reported also among mild CTEPH cases. ${ }^{479}$ As a cause for such a course, the involvement of apparent or inapparent recurrence of acute PTE cannot be ruled out, but the involvement of the thrombus formation mechanism in situ has also been suggested. Therefore, long-lasting anticoagulant therapy (warfarin) is needed regardless of the presence/ absence of interventional treatment (PEA, BPA) in CTEPH. The warfarin dose level is often adjusted to keep the prothrombin time-international normalized ratio (PT-INR) in the range 1.5-2.5, in a manner similar to that adopted for management of acute PTE. However, there is clear-cut evidence supporting the effectiveness of anticoagulant therapy. Furthermore, there is no evidence for the efficacy or safety of direct oral anticoagulants (DOAC) in cases of CTEPH.

\section{4.6.4 Treatment of Right-Sided Heart Failure}

Right-sided heart failure is an important factor determining the prognosis of CTEPH. In cases of right-sided heart failure presenting with hydrothorax, hepatomegaly, hepatic dysfunction, thrombopenia, and leg edema, conventional methods of heart failure treatment are performed, including keeping still, salt intake restriction, use of diuretics, and use of oral cardiotonics. In severe cases, serial intravenous infusion of catecholamines may also be needed.

\section{| 4.6.5 Oxygen Therapy}

Oxygen therapy is expected to alleviate symptoms (by improving oxygen transport) and to reduce the pulmonary artery pressure to a certain extent by resolving hypoxic pulmonary vasoconstriction. Although no definite evidence is available, this therapy is additionally expected to improve the prognosis. In Japan, home oxygen therapy (HOT) for pulmonary hypertension is covered by health insurance, including also patients with CTEPH.

\subsection{Balloon Pulmonary Angioplasty (BPA)}

BPA is aimed at mechanically resolving the stenoses and obstructions of the pulmonary arteries by using balloon catheters. The first case series of BPA was reported in 2001, confirming its usefulness. ${ }^{\mathbf{4 8 0}}$ However, lung injury developed frequently after reperfusion of the treated site, and the outcome of the treatment was not superior to the efficacy and safety of PEA achieved at well-experienced facilities. For this reason, in Western countries, BPA has not been emphasized as an alternative treatment for CTEPH. In Japan, where the incidence of peripheral type CTEPH is high and many cases are not eligible for surgical treatment, attempts have been continued to establish BPA as an alternative treatment for CTEPH.

Indications of BPA are as follows: (1) cases ineligible for PEA (i.e., patients with surgically inaccessible lesions, cases judged as ineligible for PEA because of advanced age or comorbidities, or patients with residual pulmonary hypertension after PEA), and (2) patients with CTEPH presenting with symptoms of NYHA/WHO functional class III or severer despite medical treatment and satisfying the other requirements, i.e., (3) expressing the desire (by the patient or family member) to receive BPA after being sufficiently informed as to the condition, risks and benefits and (4) without severe multiple organ failure. ${ }^{481}$ Because CTEPH involves lesions of varying severity levels affecting almost all segmental pulmonary arteries, treatment of all lesions during single procedure is impossible, and 4 to 6 sessions of procedure are needed per case to complete treatment. According to the report from a survey of cardiovascular disease treatment in $2016,{ }^{482}$ BPA was performed 1,910 procedures across Japan in 2015. This allows us to estimate that at least about 320 patients received BPA during that year in Japan.

Regarding the details of treatment methods, complications, and treatment outcome, reference should be made to 
"Statements about the indications and procedure of balloon pulmonary angioplasty for chronic pulmonary artery thromboembolism" published by the Japanese Circulation Society. ${ }^{481}$ The minimum goal of BPA is to resolve the pulmonary hypertension to prevent the occurrence of rightsided heart failure and to improve the prognosis (survival), as is the goal of PEA. ${ }^{483}$ The extent of improvement in pulmonary hemodynamics achieved by BPA according to Japanese reports is comparable to that achieved by PEA, 434 436 indicating that it is possible to achieve the above-mentioned goal. On the other hand, the extent of hemodynamic improvement following BPA is not sufficient according to overseas reports. ${ }^{\mathbf{4 8 4}, 485}$ Comparing the sample size, this difference may be attributable to differences in skill/experience level rather than ethnic differences.

As a complication arising from BPA, postoperative lung injury was frequently reported in early days after introduc-

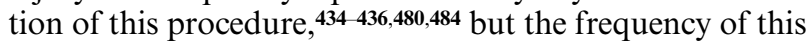
complication has been recently tending to decrease following an increase in the total number of patients receiving the procedure, ${ }^{486}$ and changes in the treatment procedure. ${ }^{487}$ However, since BPA procedure still varies among facilities, standardization of the BPA procedure is an open issue for the future. To date, no report of evident re-stenosis or recurrence of $\mathrm{PH}$ after BPA has been published, but the duration and the sample size of post-BPA follow-up are insufficient compared to the follow-up after PEA. Thus, it is another open issue to determine the extent of hemodynamic improvement immediately after treatment that may lead to favorable long-term outcome.

CTEPH is a rare disease and it is not easy to accumulate sufficient number of cases to experience sufficient number of BPA procedures. Since, CTEPH is basically chronic disease, urgent treatment is not necessary in most cases. Similar to PEA, it is necessary that BPA is performed at well-experienced facilities.

\section{4.8 Other Diseases Requiring Distinction From CTEPH}

Other than CTEPH, the following diseases can cause stenosis/obstruction of the pulmonary artery at the segmental/subsegmental level. Distinction of CTEPH from these diseases is essential.

\section{4.8.1 Primary Pulmonary Artery Sarcoma (Intimal Sarcoma)}

One of the diseases whose distinction from CTEPH is important is primary pulmonary artery sarcoma. Primary pulmonary artery sarcoma is a very rare tumor, mostly assuming the form of intimal sarcoma growing in the pulmonary artery lumen. Mural sarcoma of pulmonary artery origin is even more rare. To date, about 80 cases of this disease in Japan and about 300 cases in the world have been reported, but its actual incidence is unknown because this tumor is sometimes detected incidentally during autopsied cases. ${ }^{\mathbf{4 8 8 , 4 8 9}}$ Its prognosis varies among reports, but reports demonstrating a favorable 5-year survival rate (about 50\%) after surgical resection have also been published sporadically. The tumor often develops at age 40 and over, with the incidence among females 1.3-2.0 times as high as that among males. ${ }^{490,491}$ It presents with unspecific symptoms such as cough, hemoptysis, dyspnea and chest pain. The granuloma grows on the lumen inside the pulmonary artery, and it is often mistaken as CTEPH.

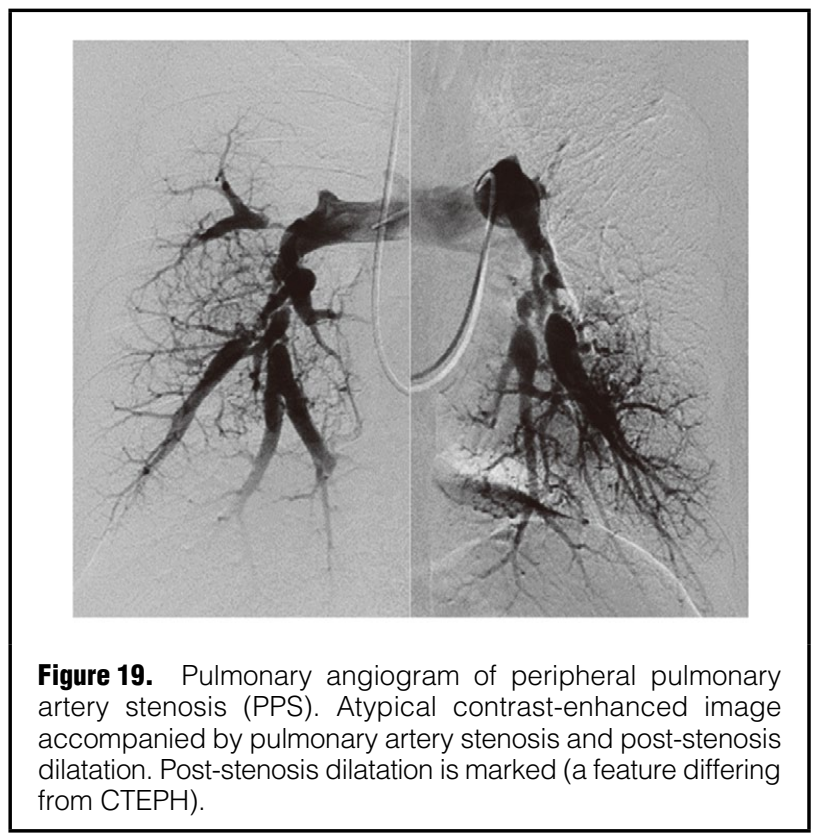

Thus, accurate differential diagnosis is essential. Diagnostic imaging, such as contrast-enhanced CT, MRI, and PET, is useful. In addition, contrast-enhanced CT is particularly useful for distinction from CTEPH in cases in which contrast is enhanced in a non-homogenous manner ${ }^{490}$ or cases in which irregular edges or signs of mass (primarily in the vicinity of the right ventricular outflow tract) are revealed by contrast-enhanced CT. However, it needs to be considered that even when PET findings are negative, primary pulmonary artery sarcoma cannot be ruled out. Histopathologically, this tumor is mostly undifferentiated sarcoma or pleomorphic sarcoma (MFH type). Its differentiated types include angiosarcoma of varying morphological features, chondrosarcoma/osteosarcoma, rhabdomyosarcoma, angiosarcoma, and leiomyosarcoma. Methods of treatment performed include surgical treatment and auxiliary therapies (chemotherapy, radiotherapy). In operable cases, complete resection, including the pulmonary valve and the right ventricular outflow tract, is recommended, with a significant difference in 5-year survival rate being reported between completely resected cases and incompletely resected cases. 488490 The outcome of uncombined treatment with chemotherapy or radiotherapy is poor, and both chemotherapy and radiotherapy are performed within the framework of multidisciplinary therapy.

\section{4.8.2 Peripheral Pulmonary Artery Stenosis (PPS)}

Peripheral pulmonary artery stenosis (PPS) involves stenosis and obstruction of the pulmonary artery trunk, bilateral main pulmonary artery, or peripheral pulmonary artery. There are numerous reports concerning PPS during childhood, which is often accompanied by various congenital diseases. Recently, the presence of patients with adulthood PPS was revealed. ${ }^{42}$ Of all cases of PPS, cases without accompanying congenital disease are called "pure type PPS." There is no report based on sufficient data concerning efficacy of pulmonary vasodilators against PPS.

Kreutzer et al. reported 12 adults with PPS. ${ }^{492}$ Their age ranged from 17 to 51 (36.2 \pm 9.7 years). Symptoms were dyspnea and malaise. In all cases, ventilation/perfusion lung 


\begin{tabular}{|c|c|c|c|c|}
\hline \multicolumn{2}{|c|}{ Table 28. Recommendation and Evidence Level Concerning Pulmonary Vasodilators for Children With IPAH/HPAH } \\
\hline $\begin{array}{c}\text { Recommendation } \\
\text { Class }\end{array}$ & $\begin{array}{c}\text { Evidence } \\
\text { Level }\end{array}$ & $\begin{array}{c}\text { NYHA/WHO } \\
\text { functional class I/II }\end{array}$ & $\begin{array}{c}\text { NYHA/WHO } \\
\text { functional class III }\end{array}$ & $\begin{array}{c}\text { NYHA/WHO } \\
\text { functional class IV }\end{array}$ \\
\hline I & B & $\begin{array}{c}\text { Bosentan po } \\
\text { Sildenafil po }\end{array}$ & $\begin{array}{c}\text { Bosentan po } \\
\text { Sildenafil po } \\
\text { Epoprostenol iv }\end{array}$ & Epoprostenol iv \\
Ila & C & $\begin{array}{c}\text { Ambrisentan po } \\
\text { Tadalafil po } \\
\text { Tadalafil po } \\
\text { Treprostinil sc }\end{array}$ & $\begin{array}{c}\text { Beraprost po } \\
\text { Iloprost inh } \\
\text { Treprostinil iv }\end{array}$ & $\begin{array}{c}\text { ERA po } \\
\text { PDE5 inhibitor po } \\
\text { Iloprost inh } \\
\text { Treprostinil sc/iv } \\
\text { Upfront combination therapy }\end{array}$ \\
\hline Upfront combination therapy
\end{tabular}

ERA: ambrisentan, bosentan. PDE5 inhibitor: tadalafil, sildenafil. po: oralinh: inhalation, iv: intravenous, sc: subcutaneous.

scan revealed multiple segmental defects, and pulmonary angiography disclosed atypical stenosis of the segmental/ subsegmental branches at several sites of both lungs. BPA was performed in 11 of the 12 cases, resulting in success in operative procedure in 9 cases (one died after surgery) and continued alleviation of symptoms (NYHA class I-II) in 7 cases. In 6 of the 12 cases, the initial diagnosis was CTEPH, but a definite diagnosis of PPS was later made on the basis of pulmonary angiography at experienced facilities.

Yanagisawa et al. performed BPA in 7 patients with PPS and 4 patients with pulmonary arteritis-associated Takayasu's disease, reporting that improvement was seen in $\mathrm{mPAP}(46 \mathrm{mmHg} \rightarrow 34 \mathrm{mmHg}), \mathrm{PVR}(10.5 \mathrm{Wood}$ Unit $\rightarrow$ 4.6 Wood Unit), and 6-minute walk distance $(371 \mathrm{~m} \rightarrow 416 \mathrm{~m})$ and that evaluation of lesions by imaging devices, such as optical coherence tomography (OCT), in addition to angiography was useful in planning the treatment. ${ }^{493}$ This report additionally comments that the response to BPA by these patients was lower than that known among CTEPH patients, suggesting the possible involvement of re-stenosis and other factors as a background.

Although adulthood PPS is rare, its distinction from CTEPH (PAH in some cases) is essential, and PAG is indispensable for its definite diagnosis (Figure 19). Although this is empirical advice, the presence of PPS should be suspected in cases of PH developing at age 20-39, cases of pulmonary hypertension accompanied by congenital heart disease, cases presenting with atypical stenosis accompanied by post-stenosis dilatation revealed by PAG (upper lobe branch is often obstructed), and cases accompanied by other types of vascular stenosis.

\section{4.8.3 Pulmonary Arteritis Associated With Takayasu's Disease}

Vasculitis associated with Takayasu's disease is classified as large vessel vasculitis. It develops frequently in young females in South East Asia. It is an inflammatory disease which causes stenosis or obstruction of the aorta, its proximal bifurcation, coronary artery, pulmonary artery, and other vessels. Takayasu's disease, which presents with only lesions of the pulmonary artery, is a rare type of this kind of disease. Complication by pulmonary artery lesions is reported to be seen in $15 \%$ of all patients with Takayasu's disease, affecting the pulmonary artery trunk to the central side (e.g., the proximal part of segmental branches). There is a report that complication by pulmonary hypertension was seen in $27 \%$ of patients with Takayasu's disease, but details are unknown. ${ }^{494}$ Histological findings include fibrous hypertrophy of the tunica intima, hypertrophy of the tunica media accompanied by destruction due to elastic fiber inflammation, and belt-shaped fibrosis of the tunica externa. Pulmonary arteritis associated with Takayasu's disease is diagnosed by PAG, CT and MRI. The findings from contrast-enhanced CT and PAG are different from the angiographic findings of CTEPH in terms of absence of parietal thrombus. At the stenosed site, full-circumferential wall hypertrophy due to connective tissue-like lesions is observed, whereas the post-stenosis periphery is characterized by preservation of relatively intact vascular structure. Care needs to be taken of the fact that findings similar to those from CTEPH are obtained by ventilation/perfusion lung scan. The diagnosis and treatment of this disease should be performed in by specialists in rheumatology. Evaluation of the disease activity is important. In cases of high activity Takayasu's disease, treatment with steroid and immunosuppressors at a facility providing expert care is considered to suppress inflammation. If the pulmonary artery lesion is irreversible, catheter surgery or bypass surgery is considered.

\subsubsection{Vasculitis Associated With Behcet's Disease}

Vasculitis associated with Behcet's disease is classified as large vessel vasculitis. Its distinction from CTEPH is sometimes difficult. Patients with Behcet's disease are reportedly distributed along the Silk Road, but the diseases complicating this disease vary among different districts. In the Far East, complication by intestinal disease is often seen, whereas complication by large vessel vasculitis is frequent $(1-7.7 \%)$ in the Middle East (Turkey and other countries). ${ }^{495}$ It often causes hemoptysis and is occasionally accompanied by pulmonary artery thromboembolism, pulmonary aneurysm, and thrombosis of the right side of the heart and veins. MRI reveals contrast enhancement. For treatment, immunosuppressors are used in addition to anticoagulants, with some reports demonstrating a response rate of $75 \% .496$

\section{Pulmonary Hypertension Due to Unclear Multifactorial Mechanisms}

As shown in Table 4, Group 5 consists of diseases involving pulmonary hypertension by several factors. A common feature of these diseases is that the mechanisms for onset of pulmonary hypertension have been poorly understood. Therefore, these patients need careful diagnosis. Further- 


\begin{tabular}{|c|c|c|}
\hline \multicolumn{3}{|c|}{$\begin{array}{l}\text { Table 29. Recommendation and Evidence Level Concerning } \\
\text { General Measures for Children With IPAH/HPAH }\end{array}$} \\
\hline Recommendation & $\begin{array}{c}\text { Recommendation } \\
\text { Class }\end{array}$ & $\begin{array}{c}\text { Evidence } \\
\text { Level }\end{array}$ \\
\hline $\begin{array}{l}\text { Timely counseling for girls during } \\
\text { adolescence about pregnancy- } \\
\text { related serious maternal/fetal risk } \\
\text { and safe contraception }\end{array}$ & I & B \\
\hline $\begin{array}{l}\text { Multidisciplinary comprehensive } \\
\text { evaluation and treatment by } \\
\text { referral of pediatric IPAH/HPAH } \\
\text { patients (including suspected } \\
\text { cases) to experienced centers }\end{array}$ & I & C \\
\hline $\begin{array}{l}\text { Appropriate monitoring by } \\
\text { physicians familiar with pediatric } \\
\text { cardiac anesthesia or intensive } \\
\text { care during surgery or invasive } \\
\text { manipulation requiring sedation } \\
\text { or general anesthesia }\end{array}$ & 1 & C \\
\hline $\begin{array}{l}\text { Avoidance of dehydration, with } \\
\text { appropriate fluid therapy } \\
\text { administered as needed }\end{array}$ & I & C \\
\hline $\begin{array}{l}\text { All vaccinations for prevention of } \\
\text { exacerbation due to preventable } \\
\text { infection, unless contraindicated }\end{array}$ & I & C \\
\hline $\begin{array}{l}\text { Participation in competitive } \\
\text { sports or hard isometric exercise }\end{array}$ & III & $\mathrm{C}$ \\
\hline $\begin{array}{l}\text { Air travel by patients other than } \\
\text { appropriately managed patients } \\
\text { in stable condition }\end{array}$ & III & $\mathrm{C}$ \\
\hline
\end{tabular}

more, the therapeutic strategy for this group varies depending on the underlying disease. Although some of the cases from this group may have pulmonary arterial hypertension (PAH), priority should not be given to the treatment of pulmonary hypertension with cases of this group. No results of randomized controlled trial (RCT) supporting the effectiveness of drugs for treatment of pulmonary hypertension with Group 5 cases have been reported.

Group 5 has been divided into four subgroups: (1) hematological disorders (chronic hemolytic anemia, myeloproliferative disorders, splenectomy), (2) systemic disorders (sarcoidosis, pulmonary histiocytosis, lymphangioleiomyomatosis, neurofibromatosis, vasculitis), (3) metabolic disorders (glycogen storage disease, Gaucher disease, thyroid disorders), and (4) other conditions (tumoral thrombotic microangiopathy, fibrosing mediastinitis, chronic renal failure, segmental pulmonary hypertension). Association of pulmonary hypertension is frequently seen in patients with chronic hemolytic anemia, myeloproliferative disorders. Although chronic hemolytic anemia (e.g., sickle cell anemia, thalassemia, and hereditary spherocytosis) was included in Group 1 according to the Dana Point Classification (2008), they were transferred to Group 5 in the Nice Classification (2013) because the etiology of pulmonary hypertension involves several factors such as chronic thromboembolism, splenectomy, increased cardiac output, hyperviscosity, and left heart disease.

\section{Pulmonary Hypertension in Children}

\subsection{Idiopathic/Heritable Pulmonary Arterial Hypertension (IPAH/HPAH) \\ Summary}

There is no sufficient evidence that can be the rationale

\begin{tabular}{|l|c|c|}
\hline \multicolumn{1}{|c|}{ Table 30. Recommendation and Evidence Level Concerning } \\
Supportive Therapy for Children With IPAH/HPAH
\end{tabular}

when guidelines on pulmonary hypertension in children are prepared. Also the knowledge/suggestion concerning the diagnosis/treatment of idiopathic/heritable pulmonary arterial hypertension (IPAH/HPAH) in children is often based on experts' consensus views or small-scale or retrospective studies, and their evidence level is not high (mostly Level C). ${ }^{1}$ As of February 2018, only three drugs including bosentan (Tracleer Dispersible Tablet for Pediatric ${ }^{\circledR}$ ), epoprostenol (Epoprostenol "ACT"®), and sildenafil (Revatio Tablet ${ }^{\circledR} \cdot$ OD Film ${ }^{\circledR}$. Dry Syrup for Suspension ${ }^{\circledR}$ ), have been approved for pediatric pulmonary arterial hypertension in Japan. Because pediatric use of most drugs has not been covered by health insurance, they have been used in the form of "off-label use" and their drug information on usage, dosage, and safety have not been established. However, because Japan has recently begun to participate in global cooperative clinical studies on this kind of drug and because clinical studies in children have also begun to be carried out in Japan, we may expect expansion of the indications of this kind of drug to include pediatric use. Recommendation of pulmonary vasodilators to treat pediatric IPAH/HPAH (by severity level) is shown in Table 28 and general measures and supportive therapy are given in Table 29 and Table 30.

\subsubsection{Epidemiology/Etiology}

Little information is available concerning the epidemiology of pediatric pulmonary hypertension in Japan. According to the nationwide survey of primary pulmonary hypertension during childhood (corresponding to the current concept "IPAH/HPAH") by Saji et al., ${ }^{497}$ the annual incidence is approximately 1 out of one million population, and children are estimated to account for about $25 \%$ of all patients with $\mathrm{PH}$, including adults. According to an epidemiological study in the United Kingdom, ${ }^{498}$ the annual incidence of 


\begin{tabular}{|c|c|c|}
\hline Lower risk & Prognostic factor & Higher risk \\
\hline No & Signs of right ventricular failure & Yes \\
\hline No & Disease progression & Yes \\
\hline \multirow[t]{2}{*}{ No } & Syncope & Yes \\
\hline & Growth & Poor \\
\hline I, II & NYHA/WHO functional class & III, IV \\
\hline Minimally increased & BNP/NT-proBNP & Significantly increased \\
\hline Long (>500 m) & 6-minute walk distance & Short $(<300 \mathrm{~m})$ \\
\hline $\begin{array}{l}\text { Mild right ventricular } \\
\text { enlargement/dysfunction }\end{array}$ & Echocardiography & $\begin{array}{l}\text { Marked right ventricular } \\
\text { enlargement/dysfunction } \\
\text { Pericardial effusion }\end{array}$ \\
\hline $\begin{array}{c}\mathrm{Cl}>3.0 \mathrm{~L} / \mathrm{min} / \mathrm{m}^{2} \\
\mathrm{mPAP} / \mathrm{mSAP}<0.5 \\
\mathrm{PVRI}<10 \text { Wood Unit. } \mathrm{m}^{2} \\
\text { Good acute vasoreactivity }\end{array}$ & $\begin{array}{c}\text { Hemodynamics } \\
\text { (Cardiac catheterization) }\end{array}$ & $\begin{array}{c}\mathrm{Cl}<2.5 \mathrm{~L} / \mathrm{min} / \mathrm{m}^{2} \\
\mathrm{mPAP} / \mathrm{mSAP}>0.75 \\
\mathrm{RAP}>15 \mathrm{mmHg} \\
\text { PVRI }>15 \text { Wood Unit } \cdot \mathrm{m}^{2}\end{array}$ \\
\hline
\end{tabular}

(Source: Prepared based on Hansmann G, et al. 2016509)

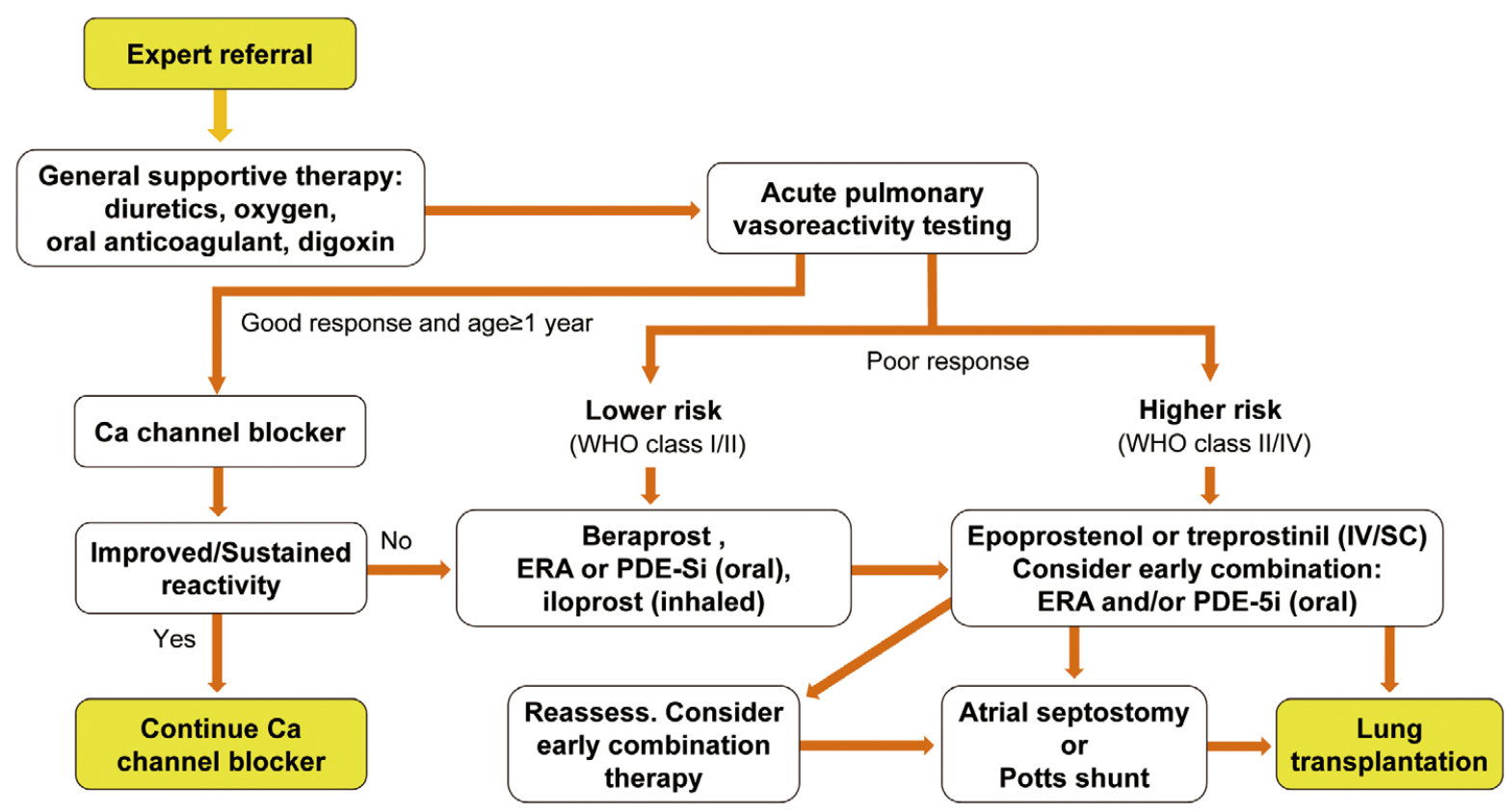

Figure 20. Algorithm for pediatric IPAH/HPAH treatment. (Source: Prepared based on Hansmann G, et al. 2016509)

IPAH at age less than 16 is 0.48 out of one million population and the prevalence of this disease is 2.1 out of one million population. In the Netherlands, the annual incidence of IPAH has been reported to be 0.7 out of one million population, with the incidence of $\mathrm{PAH}$ due to congenital heart disease being 2.2/1 million population and the prevalence of PAH due to congenital heart disease being 15.6/1 million population. ${ }^{499}$ According to an epidemiological study of pulmonary hypertension among children aged less than 18 at 31 facilities of 19 countries including Japan (TOPP Registry), ${ }^{\mathbf{5 0 0}}$ analysis by cause revealed that an overwhelming majority was accounted for by IPAH/ HPAH (57\%) and pulmonary hypertension associated with congenital heart disease $(36 \%)$, followed by pulmonary hypertension due to bronchopulmonary dysplasia (BPD).
It was additionally shown that chromosomal abnormalities, such as 21-trisomy, was present in 13\% of all cases.

Following recent advances in molecular cytological studies, it has been revealed that the onset of PAH involves interactions between hereditary disposition and risk factors and that major factors responsible for $\mathrm{PAH}$ are endothelial cell dysfunction (primarily inflammation/degeneration/ proliferation of pulmonary arterioles), resistance of smooth muscle cells to apoptosis, and vascular wall hypertrophic changes and remodeling (by uncontrolled cell proliferation). In cases of IPAH/HPAH, mutation of genes $B M P R 2$, ALK1, ALK6, and Endoglin as well as intracellular signal gene Smad8 mutation is seen in $75 \%$ of all familial-onset cases and $20-25 \%$ of isolated (idiopathic) onset cases. This disease is inherited in an autosomal dominant pattern, but 


\begin{tabular}{|c|c|c|c|}
\hline Drug & $\begin{array}{l}\text { Endothelin receptor antagonist: } \\
\text { Bosentan }\end{array}$ & $\begin{array}{c}\text { Phosphodiesterase } 5 \text { inhibitor: } \\
\text { Sildenafil }\end{array}$ & $\begin{array}{l}\text { Prostaglandin } I_{2} \text { preparation: } \\
\text { Epoprostenol }\end{array}$ \\
\hline Brand name & $\begin{array}{l}\text { Tracleer }{ }^{\circledR} 32 \mathrm{mg} \text { Dispersible Tablets } \\
\text { for Pediatric }\end{array}$ & $\begin{array}{l}\text { Revatio }{ }^{\circledR} \text { Tablets } 20 \mathrm{mg}, \\
\text { Revatio }{ }^{\circledR} \text { OD Film } 20 \mathrm{mg}, \\
\text { Revatio }^{\circledR} \text { Suspension Dry Syrup } 900 \mathrm{mg}\end{array}$ & $\begin{array}{l}\text { Epoprostenol ACT } 0.5 \mathrm{mg} / 1.5 \mathrm{mg} \text { for } \\
\text { Intravenous Injection }\end{array}$ \\
\hline Formulation & $\begin{array}{l}\text { Tablets with a cruciate cut line; can } \\
\text { be divided into } 1 / 2 \text { or } 1 / 4 \text { portions }\end{array}$ & $\begin{array}{l}\text { OD film: Possible to ingest with saliva } \\
\text { alone (without water) } \\
\text { Suspension: Water }(90 \mathrm{~mL} \text { ) is added to } \\
\text { one bottle, to yield } 10 \mathrm{mg} / \mathrm{mL} \text { solution }\end{array}$ & $\begin{array}{l}\text { Dissolved with special solvent } \\
\text { (physiological saline) }\end{array}$ \\
\hline Indications & Children (age $\geq 1$ year) with $\mathrm{PAH}$ & $\begin{array}{l}\text { Children (age } \geq 1 \text { year) with } \mathrm{PAH} \\
\text { Body weight } \geq 8 \mathrm{~kg}\end{array}$ & Children (age $\geq 1$ year) with $\mathrm{PAH}$ \\
\hline $\begin{array}{l}\text { Administration/ } \\
\text { Dosage }\end{array}$ & $\begin{array}{l}2-4 \mathrm{mg} / \mathrm{kg} / \text { dose, twice daily (dosage } \\
\text { halved during first } 4 \text { weeks), } \\
\text { maximum } 120 \mathrm{mg} / \text { dose }(240 \mathrm{mg} / \text { day) }\end{array}$ & $\begin{array}{l}\text { Body weight } 8-20 \mathrm{~kg}: 10 \mathrm{mg} / \text { dose, three } \\
\text { times daily } \\
\text { Body weight } \geq 20 \mathrm{~kg}: 20 \mathrm{mg} / \text { dose, three } \\
\text { times daily }\end{array}$ & $\begin{array}{l}\text { Continuous intravenous infusion, with } \\
\text { initial rate } 0.5-2 \mathrm{ng} / \mathrm{kg} / \mathrm{min} \text {. Dosing } \\
\text { rate increased by } 0.5-2 \mathrm{ng} / \mathrm{kg} / \mathrm{min} \text { at } \\
\text { intervals of } 1-4 \text { weeks, to determine } \\
\text { optimum dosing rate (around } \\
20-40 \mathrm{ng} / \mathrm{kg} / \mathrm{min} \text { ) }\end{array}$ \\
\hline $\begin{array}{l}\text { Major adverse } \\
\text { reactions }\end{array}$ & Headache, liver dysfunction, myalgia & $\begin{array}{l}\text { Headache, flushing, abdominal pain, } \\
\text { nausea }\end{array}$ & $\begin{array}{l}\text { Headache, flushing, diarrhea, jaw } \\
\text { pain, thrombocytopenia }\end{array}$ \\
\hline $\begin{array}{l}\text { Features in } \\
\text { children }\end{array}$ & $\begin{array}{l}\text { Liver dysfunction caused less } \\
\text { frequently than in adults }\end{array}$ & $\begin{array}{l}\text { Spontaneous penile erection reported } \\
\text { also in children }\end{array}$ & $\begin{array}{l}\text { Can cause hypotension by vasodilating } \\
\text { action. Careful monitoring and } \\
\text { consider intravenous fluid infusion } \\
\text { and catecholamine as needed. } \\
\text { Hyperthyroidism reported also in } \\
\text { children, requiring periodical exams }\end{array}$ \\
\hline
\end{tabular}

the penetrance rate is low $(10-20 \%)$. On the whole, the age upon onset of PAH is lower in cases positive as to mutation of these genes than in mutation negative cases, and hemodynamic disorders are severer and the prognosis is less favorable in the mutation positive group. ${ }^{501}$

\section{| 6.1.2 Diagnosis}

Compared to IPAH/HPAH in adults, IPAH/HPAH in children can be characterized by:

- Well-preserved cardiac index and low right atrial pressure despite high pulmonary artery pressure and pulmonary/ systemic blood pressure ratio

- Unlikely to present facial/leg edema even in moderate to severe cases because of preserved distensibility in systemic vein

- Likely to develop imbalance of oxygen demand and supply during excessive exercise, resulting in marked dyspnea and syncope..$^{502}$

The diagnostic method of pulmonary hypertension in children is almost similar to that of adults, and is based on the PH diagnosis algorithm (see Figure 1). This algorithm includes tests difficult to perform to infant and young children (e.g., pulmonary function test, 6-minute walk test, and cardiopulmonary exercise testing), but it is necessary to conduct these tests and evaluate the results before the start of treatment. The gold standard for a definite diagnosis of pulmonary hypertension is cardiac catheterization. However, since the incidence of severe complications induced by catheterization is higher in infants and small children than elderly children and adults, careful consideration is needed as to implementation of this test. ${ }^{503-505}$ Particularly in infants and small children of NYHA/WHO functional class IV, invasive tests should be avoided for a reason of high risk and, if echocardiography allows a tentative diagnosis of Group $1(\mathrm{PAH})$, it is advisable to give priority to therapeutic intervention using specific pulmonary vasodilators, postponing cardiac catheterization until stabilization of the condition. If cardiac catheterization is performed, sufficient care needs to be taken of safety, including arranging various methods of monitoring at facilities with specialists and taking appropriate measures for sedation. Therefore, in children, echocardiography is of high value and is useful not only in screening of pulmonary hypertension but also in morphological and functional diagnosis of the right ventricle and chronological evaluation. ${ }^{506}$

\subsubsection{Prognosis/Severity Rating}

\section{a. Prognosis}

Before 2000, no effective drug for treatment of this disease was available, and the prognosis of patients with IPAH/ HPAH was quite poor. According to a report from the United States, the prognosis of pediatric IPAH/HPAH cases was quite poor (mean survival period 10 months) compared to adults with IPAH/HPAH (mean survival period 2.8 years), with most children dying within $1-2$ years after the diagnosis of IPAH/HPAH. ${ }^{82}$ In recent years, specific pulmonary vasodilators, such as epoprostenol, were introduced clinically, and long-term survivors responding well to treatment with various drugs have been increasing, allowing us to expect prognosis after treatment in pediatric IPAH/HPAH cases not inferior to the prognosis in adult cases. Among the results reported recently, analysis of the data from 216 patients aged 18 or less registered with the REVEAL Study (a large-scale registry study in the USA) revealed a 5-year survival rate of $74 \%$ after the diagnosis of IPAH/HPAH. ${ }^{507}$

\section{b. Severity/Prognosis Evaluation}

Indicators important for severity/prognosis evaluation of children with IPAH/HPAH ${ }^{508,509}$ are shown in Table 31. Patients with many factors counted as indicators of favorable prognosis are low-risk cases and cases poor in such factors are rated as high-risk cases when therapeutic strategy is determined.

WHO functional classification is designed for adults, 
and evaluation with this classification is difficult in infants/ small children and children with growth retardation (chromosomal aberration). It is necessary to conduct evaluation of individual cases by assessing the status of attendance to nursery school, kindergarten, or school as well as presence/ absence of problems with play and exercise. ${ }^{509}$

The 6-minute walk test is useful in severity rating and prediction of the outcome in children with pulmonary hypertension, as is the case with adults with pulmonary hypertension. In children with IPAH aged 7 years and over and children with $\mathrm{PAH}$ accompanied by congenital heart disease, the walk distance reflects the severity of disease and the magnitude of reduction in percutaneous oxygen saturation during walk served as an additional prognostic factor after adjustment for the presence/absence and extent of shunt. 510

Genetic test requires ethical consideration before implementation and is hence not indispensable. However, the results of this test are of value as reference information, because cases positive as to mutation of gene $B M P R 2$ and $A L K 1$ are known to have poor prognosis compared to negative cases. ${ }^{511}$

Tissue Doppler ultrasound study is useful in severity rating of children with IPAH/HPAH. Tricuspid valve e' correlated with disease severity and $\mathrm{e}^{\prime}<8 \mathrm{~cm} / \mathrm{sec}$ is used as an indicator for prediction of the risk of hospitalization/ lung transplantation/death for a reason of heart failure. ${ }^{512}$

\section{6.1.4 Guidelines on Treatment}

Guidelines on diagnosis and treatment of pediatric pulmonary hypertension were published in 2015 (USA) and 2016 (Europe). ${ }^{508,509}$ These guidelines were prepared on the basis of the treatment algorithm for adults and differ little in contents between the American guidelines and the European guidelines. The pediatric IPAH/HPAH treatment algorithm modified on the basis of these overseas guidelines, reflecting the current status in Japan, is shown in Figure 20 and the recommendation of pulmonary vasodilators in the treatment of pediatric IPAH/HPAH (by severity level) is given in Table 28. Furthermore, general measures and supportive therapy for children with IPAH/HPAH are summarized in Table 29 and Table 30. Regarding treatment of pediatric cases, evidence level for pulmonary vasodilators is not high (B or C) even when the recommendation class is I. The drugs listed in this table include some drugs not covered by health insurance as to use in children in Japan, but these drugs have been administered to children with IPAH/ HPAH during routine clinical practice on the basis of the characteristics and available domestic/overseas findings in the field of pediatrics on each drug and have been contributing greatly to improving the prognosis to date. ${ }^{513}$ In Japan, beraprost (a prostaglandin derivative) is often used, primarily in mild cases, and has shown efficacy, but its evidence level is not high. ${ }^{513}$ Sildenafil is excellent in terms of pulmonary vessel selectivity and highly convenient, but there is a report concerning the potential danger of increased risk for death at high dose level $(>3 \mathrm{mg} / \mathrm{kg} / \mathrm{day}),{ }^{514}$ thus requiring caution as to its dose level. A list of drugs covered by health insurance in Japan as to pediatric use is given in Table 32.

Lung transplantation should be considered in cases resisting maximum medical treatment, including intravenous prostacyclin treatment. Such cases should be referred to facilities providing lung transplantation, as desired, at appropriate timing. Palliative Potts shunt operation
(Recommendation Class IIb, Evidence Level C) can be a substitute for lung transplantation in children with severe IPAH presenting with marked pulmonary hypertension exceeding the systemic blood pressure, although little experience with this operation is available in Japan. During this operation, a right-to-left shunt is created by side-toside anastomosis of the descending aorta to the left pulmonary artery. As a result, the magnitude of reduction in percutaneous arterial oxygen saturation $\left(\mathrm{SpO}_{2}\right)$ of upper extremities is small (reduction to $94 \%$ ) relative to the reduction in lower extremities (reduction to $83 \%$ ), allowing long-term survival and preservation of functional class I in 6 of the 8 patients. ${ }^{515}$ This palliative operation needs to be performed by an experienced heart surgeon but it may be advantageous for maintaining oxygen supply to the brain compared to atrial septostomy (Recommendation Class IIa, Evidence Level C).

\subsubsection{Patient Management}

Recently, long-term survival can be expected of patients responding well to various specific pulmonary vasodilators. However, in rapidly progressive cases and cases resisting drug therapy, referral to facilities with specialists at early occasions is recommended. Also, during the stable stage, periodical assessment of the condition and hemodynamics should be performed and the treatment methods need to be reviewed as needed.

Precautions in patient management during daily living are similar to those for adults with IPAH/HPAH. Particularly for infants and small children, care is needed of possible acute exacerbation upon infection (of respiratory system, gastrointestinal system). In winter, vaccination of the patient and family members against influenza should be recommended. During fever, antipyretic treatment and oxygen inhalation should be performed to avoid loss of oxygen demand-supply balance.

In Japan, the school daily life management guidance table has been used for management of schoolchildren with heart disease. Under this system, attendance of the sick child to school and participation in physical exercise and extracurricular activities are decided on the basis of the management category determined with consideration of the disease severity level. Exercise by children with IPAH/HPAH involves the risk for exacerbation and syncope. Even in cases in which the NYHA/WHO functional class is rated as I or II at the time of diagnosis, physical exercise should be limited to watching the exercise lesson or participating in only mild exercise, as a rule, and observation/evaluation should be repeated at intervals of 1-2 months.

As a result of an increase in the number of long-surviving children with pulmonary hypertension following advances in treatment, reports have been published concerning cases presenting with abnormal thyroid function, including asymptomatic cases (thyroid-related antibody positive) during the course of management. ${ }^{516,517}$ It has been noted for many years that PAH often develops in juvenile and middle-aged females and that complication by thyroid disease, such as Graves' disease and Hashimoto's disease, is seen frequently in adults with PAH. In children, such complications have been considered to be relatively rare. However, care needs to be taken of possible complication by abnormal thyroid function also in children with PAH. In addition, association of such complication with prolonged serial intravenous infusion of epoprostenol has 
Table 33. Recommendations on the Evaluation and Management of Pulmonary Hypertension (PH) in Children and Adolescents With Congenital Heart Disease (PAH-CHD, PPHVD-CHD)

Recommendation

Children/adolescents with clinically confirmed CHD should undergo specific transthoracic echocardiographic (TTE) screening for pulmonary arterial hypertension (PAH) and/or ventricular dysfunction. TTE cannot reliably distinguish between PAH with increased PVR (PPHVD) and without increased PVR

In children and adolescents with PAH/PPHVD-CHD, a complete diagnostic workup needs to be performed in order to determine whether $\mathrm{PAH}$ is associated or causally related to concomitant $\mathrm{CHD}$

Operability/catheter intervention: surgery or interventional closure for CHD with simple post-tricuspid

shunts (VSD, PDA) and significant left-to-right shunting should preferably be performed within the first six months of life

Children with PAH-CHD, significant left-to-right shunting and uncertainties regarding the PVR are recommended to undergo comprehensive right and left heart catheterization regardless of the patient's age

Operability: children with CHD and simple defects (VSD, PDA) beyond the typical date of surgery (see main text) or those with shunts and cyanosis should undergo comprehensive right and left cardiac catheterization

Children with PVRI $<6$ WU $\times \mathrm{m}^{2}$ and a PVR/SVR ratio $<0.3$ in the absence of additional risk factors are eligible for standard management/surgery/percutaneous interventional device closure

Children with $\mathrm{PVRI} \geq 6 \mathrm{WU} \times \mathrm{m}^{2}$ and a PVR/SVR ratio $\geq 0.3$ have to be evaluated by acute vasodilative testing (AVT)

Operability in complex CHD has to be judged individually, considering age, size and type of lesions and associated syndromes

In children/adolescents with PAH/PPHVD-CHD and left heart congestion either due to obstruction or secondary due to myocardial dysfunction, it is recommended to perform full hemodynamic evaluation by comprehensive right and left heart catheterization

Children with PAH-CHD and significant left-to-right shunting, congestive heart failure (pulmonary congestion), failure to thrive and $\mathrm{SpO}_{2}>95 \%$ (lower extremities) can be considered 'operable' for shunt closure, however, perioperative $\mathrm{PH}$ crisis may occur

In children/adolescents with single-ventricle physiology, the hemodynamic threshold for operability preFontan surgery is probably a mean TPG $\leq 6 \mathrm{mmHg}$

In clinically asymptomatic children/adolescents with single-ventricle physiology and total cavopulmonary connection (Fontan), a PVRI $\leq 3 \mathrm{WU} \times \mathrm{m}^{2}$ and mean TPG $\leq 6 \mathrm{mmHg}$ is consistent with acceptable hemodynamics

In children/adolescents with total cavopulmonary connection (Fontan) signs of increased PVRI and/or low $\mathrm{Qp}$ and/or hepatic congestion should undergo complete diagnostic workup, including comprehensive cardiac catheterization

In children/adolescents with total cavopulmonary connection (Fontan) and PHVD (TPG is $>6 \mathrm{mmHg}$ ), or those Fontan patients with symptoms irrespective of hemodynamics, targeted $\mathrm{PH}$ therapies should be considered to improve exercise capacity

In inoperable children/adolescents with Eisenmenger's syndrome, targeted pharmacotherapy as single drug or combination therapy (sequential, upfront) may be used, depending on WHO functional class and symptoms

\begin{tabular}{|c|c|}
\hline $\begin{array}{c}\text { Recommendation } \\
\text { Class }\end{array}$ & $\begin{array}{c}\text { Evidence } \\
\text { Level }\end{array}$ \\
\hline I & C \\
\hline I & C \\
\hline I & C \\
\hline I & C \\
\hline I & C \\
\hline I & C \\
\hline I & C \\
\hline Ila & C \\
\hline Ila & C \\
\hline Ila & C \\
\hline
\end{tabular}

been found, although the mechanism remains unexplained. Because thyrotoxicosis can follow a fatal course, periodical thyroid test (once in 1-2 years) is recommended.

Hemoptysis increases in frequency with time and is known to have the potential of being a direct cause for death, in addition to its association with serious prognosis. Quick actions are therefore needed to treat hemoptysis..518

\subsection{Pulmonary Hypertension Due to Congenital Heart Disease}

\section{Summary}

Pulmonary hypertension is one of the important complications determining the prognosis of congenital heart disease (CHD). It is affected not only by the type of underlying heart disease but also by many other factors including the age of sick child, location of shunt (before or after the tricuspid valve), extent of shunt, history of chronic lung disease, and presence/absence of chromosomal anomaly, malformation syndrome, and pulmonary hypertensionrelated gene mutation. Pulmonary hypertension due to
CHD includes not only Group 1 (PAH) but also Group 2 (pulmonary hypertension due to left heart disease) and Group 5 (pulmonary hypertension due to unclear multifactorial mechanisms) according to the Nice Classification (2013). In cases of PAH due to shunt disease, cure/recovery is possible by repair surgery at early stages of the disease, but treatment with pulmonary hypertension-targeting drugs is indicated in cases of Eisenmenger's syndrome presenting with irreversible pathological changes in peripheral pulmonary arterioles after persistence of left-to-right shunt for a certain period of time, cases of PAH not explained by left-to-right shunt and cases of postoperative PAH without significant residual shunt. However, most of the pulmonary hypertension-targeting drugs are not approved for children. The PAH-targeting therapy for pediatric PAH has been performed on the basis of deduction from the evidence available for adults and the views of experts. At present, a large-scale clinical study of children with PAH is being planned, and we may expect therapeutic guidelines with high evidence level in the future.

Among children having undergone surgical repair of 
Table 34. Clinical Classification of Group 1 (PAH Due to CHD)

\begin{tabular}{|l|l|}
\hline \multirow{2}{*}{ Eisenmenger syndrome } & $\begin{array}{l}\text { Includes all large intra- and extra-cardiac defects which begin as systemic-to-pulmonary shunts and prog- } \\
\text { ress with time to severe elevation of PVR and to reversal (pulmonary-to-systemic) or bidirectional shunt- } \\
\text { ing; cyanosis, secondary erythrocytosis, and multiple organ involvement are usually present }\end{array}$ \\
\hline \multirow{2}{*}{ Left-to-right shunts } & $\begin{array}{l}\text { - Correctable } \\
\text { - Noncorrectable } \\
\text { Include moderate to large defects, PVR is mildly to moderately increased systemic-to-pulmonary shunting } \\
\text { is still present, whereas cyanosis is not a feature }\end{array}$ \\
\hline PAH with coincidental CHD & $\begin{array}{l}\text { Marked elevation in PVR in the presence of small cardiac defects (left-to-right shunts), which themselves } \\
\text { do not account for the development of elevated PVR; the clinical picture is very similar to idiopathic PAH. } \\
\text { To close the defects is contraindicated }\end{array}$ \\
\hline Postoperative PAH & $\begin{array}{l}\text { Congenital heart disease is repaired, but PAH either persists immediately after surgery or recurs/develops } \\
\text { months or years after surgery in the absence of significant postoperative hemodynamic lesions. The clini- } \\
\text { cal phenotype is often aggressive }\end{array}$ \\
\hline
\end{tabular}

(Source: Simmonneau G, et al. 20138)

\begin{tabular}{|l|c|c|}
\hline \multicolumn{3}{|c|}{ Table 35. Classification of Group 2 (Pulmonary Hypertension } \\
Due to Left Heart Disease) \\
\hline \multicolumn{1}{|c|}{ Condition } & PAWP & DPD \\
\hline Isolated post-capillary PH & $>15 \mathrm{mmHg}$ & $<7 \mathrm{mmHg}$ \\
\hline $\begin{array}{l}\text { Combined post-capillary and pre- } \\
\text { capillary PH }\end{array}$ & $>15 \mathrm{mmHg}$ & $\geq 7 \mathrm{mmHg}$ \\
\hline
\end{tabular}

univentricular heart by Fontan operation, there are patients with failing pulmonary circulation ("failing Fontan" cases). For such cases, pulmonary hypertension-targeting therapy has been extensively performed in recent years by grouping them as cases of pediatric pulmonary hypertensive vascular disease (PPHVD) because of similarities in pathological changes in peripheral pulmonary arterioles, increased pulmonary vascular resistance (PVR), and responses to pulmonary hypertension-targeting drugs, although these patients are without pulmonary hypertension. The recommendation of treatment for pulmonary hypertension due to congenital heart disease in children is shown in Table 33.

\section{| 6.2.1 Epidemiology}

In the registry studies of pediatric pulmonary hypertension conducted in Western countries, IPAH/HPAH and PAH due to $\mathrm{CHD}$ (CHD-PAH) accounted for $90 \%$ of all diseases responsible for pediatric $\mathrm{PH} .{ }^{500,507,519-521}$

Underlying heart diseases with rapidly progressive pulmonary vascular lesions reported to date in children with CHD-PAH include complete transposition of great arteries with high pulmonary blood flow, truncus arteriosus, complete atrioventricular septal defect, total anomalous pulmonary venous return and univentricular heart (associated with heterotaxy syndrome). Down's syndrome is considered to involve rapid progression of pulmonary vascular lesions under the influence of several factors such as complication by airway stenosis, subpleural lung emphysema/alveolar wall hypoplasia/numerous small atelectasis (due to abnormal lung development), and pulmonary arteriolar medial smooth muscle hypoplasia/ abnormal pulmonary artery arrangement (due to abnormal development of the pulmonary artery), regardless of the presence/absence of heart disease. ${ }^{522}$

\subsubsection{Pathophysiology}

\section{a. Group 1 (CHD-PAH)}

Similar to the conventional clinical classification, Group 1 is subdivided into four subgroups: (1) Eisenmenger's syndrome, (2) cases accompanied by significant left-to-right shunt (surgically repairable cases and unrepairable cases), (3) PAH not explained by left-to-right shunt, and (4) postoperative $\mathrm{PAH}$ cases without significant residual shunt (Table 34). ${ }^{8}$

Clinical course is important for the diagnosis of Eisenmenger's syndrome, and the presence of a period characterized by high pulmonary blood flow accompanied by large-volume left-to-right shunt is known. The shear stress arising from high pulmonary blood flow stimulates vascular wall remodeling of pulmonary arterioles (see Section of Pathology for details), leading to irreversible neointima formation and increased PVR. A right-to-left shunt or bidirectional shunt induces cyanosis and secondary erythrocytosis, resulting in multiple organ failures. Regarding irreversible lesions of pulmonary arterioles, recovery in response to pulmonary hypertension-targeting drug treatment is not expected or proven. Eisenmenger's syndrome, first reported by Eisenmenger and later presented as a characteristic disease group by Paul Wood in $1958^{523}$ is a disease group defined on the basis of clinical signs, instead of being defined solely on the basis of pulmonary hemodynamics and pulmonary arteriolar lesions. These features of Eisenmenger's syndrome have not been sufficiently studied or clarified. This is the severest form of CHD-PAH and is contraindicated for repair surgery, as a rule. The survival period of children with this disease is 11.4 years, shorter than that of adult cases (30-40 years). ${ }^{499}$ Complex heart malformation is often seen as the underlying disease, in contrast to adult cases in which simple heart malformation, such as atrial septal defect, is often observed.

In cases of disease involving volume overload on the right heart, pulmonary hypertension remains or develops if repair surgery is postponed for 1-2 years or more, possibly leading to Eisenmenger's syndrome. For this reason, early surgery is recommended in cases accompanied by underlying rapidly progressive heart disease, chromosomal aberration, or malformation syndrome. Although details about the judgment as to the need of surgery are given in the Section of Diagnosis, comprehensive evaluation tailored to individual cases is needed in borderline cases because there are no widely applicable criteria for surgery at present. Following recent expansion of the alternatives for targeting drug therapy, "treat and repair" or "repair and treat" is now possible. Thus, the possibility for performing repair surgery to this group of CHD-PAH has been markedly expanding. 


\begin{tabular}{|c|l|}
\hline \multicolumn{2}{|l|}{ Table 36. Panama Classification } \\
\hline Category & \multicolumn{1}{c|}{ Description } \\
\hline 1 & Prenatal or developmental pulmonary hypertensive vascular disease \\
\hline 2 & Perinatal pulmonary vascular maladaptation \\
\hline 3 & Pediatric cardiovascular disease \\
\hline 4 & Bronchopulmonary dysplasia \\
\hline 5 & Isolated pediatric pulmonary hypertensive vascular disease (isolated pediatric PAH) \\
\hline 6 & Multifactorial pulmonary hypertensive vascular disease in congenital malformation syndromes \\
\hline 7 & Pediatric lung disease \\
\hline 9 & Pediatric thromboembolic disease \\
\hline 10 & Pediatric hypobaric hypoxic exposure \\
\hline
\end{tabular}

(Source: Cerro MJ, et al. 2011530)

In cases of PAH not explained by a left-to-right shunt, repair surgery is meaningless. Such cases should be considered as resembling IPAH/HPAH, and drug therapy with the targeting drugs is recommended.

Cases of post-repair PAH without significant residual lesions can be divided into patients with pulmonary hypertension remaining immediately after surgery and patients with $\mathrm{PH}$ recurring several months to several years after surgery. These cases should be regarded as resembling IPAH/HPAH, and drug therapy with the targeting drugs is recommended.

\section{b. Group 2 (Pulmonary Hypertension Due to Left Heart Disease)}

The Nice Classification (2013) adopted congenital/acquired left ventricular inflow tract/outflow tract obstructive disease and congenital cardiomyopathy as subgroups of Group 2. Left ventricular inflow tract obstructive disease includes pulmonary vein stenosis, cor triatriatum, mitral stenosis, parachute mitral valve, stenosing supramitral ring, and cardiac tumor (acquired myxoma/liposarcoma). Left ventricular outflow tract obstructive disease includes hypertrophic cardiomyopathy, aortic valve stenosis (including the bicuspid valve), and subaortic/aortic/supraaortic valve stenosis (Williams syndrome, rubella syndrome. ${ }^{524}$

At the Nice Conference, it was recommended to discontinue use of the expression "out of proportion $\mathrm{PH}$ " and to adopt a classification using the diastolic pulmonary pressure gradient (DPG; difference between PAWP and diastolic pulmonary artery pressure [diastolic PAP]) as an indicator associated with the presence/absence of pulmonary vascular lesions unlikely to be affected by pulmonary blood flow and pulmonary artery wedge pressure (PAWP). The normal range of DPG has been reported to be $1-2 \mathrm{mmHg}$ and DPG apparently abnormal has been reported to be $>5 \mathrm{mmHg}$. ${ }^{525}$ Therefore, the Conference proposed criteria for typing by which DPG $<7 \mathrm{mmHg}$ is rated as isolated post-capillary $\mathrm{PH}$ and $\mathrm{DPG} \geq 7 \mathrm{mmHg}$ as combined postcapillary PH and pre-capillary PH (Table 35).

\section{c. Group 5 (Pulmonary Hypertension Due to Unclear Multifactorial Mechanisms)}

The Nice Classification adopted segmental pulmonary hypertension as a subgroup of Group 5. Its underlying disease includes aortopulmonary collateral arteries (complicating the pulmonary artery atresia accompanied by ventricular septal defect [VSD]) and peripheral pulmonary artery stenosis. ${ }^{524}$

\section{d. PPHVD After Right-Sided Heart Bypass Surgery (Glenn or Fontan Operation)}

In cases of complicated CHD for which biventricular repair is not possible, right-sided heart bypass surgery by cavopulmonary anastomosis is performed, and pulmonary circulation is improved by the transpulmonary pressure difference (TPG; difference between mean pulmonary artery pressure [mPAP] and mean left atrial pressure, corresponding to the driving pressure in pulmonary circulation) and the kinetic energy provided from the systemic ventricle. For this reason, even a slight increase in PVR may affect pulmonary circulation, leading to left-sided heart failure due to reduced preload. In analysis of postoperative lung biopsy data, early signs of PAH (e.g., tunica media/ intima hypertrophy, muscularization of nonmuscular pulmonary arterioles, and thrombus formation) were noted, ${ }^{526}$ and enhanced endothelin expression ${ }^{527}$ and improved exercise tolerance following endothelin receptor antagonist treatment ${ }^{\mathbf{5 2 8 , 5 2 9}}$ were observed.

In 2011, the PVRI Pediatric Taskforce found that special features of pediatric pulmonary hypertension (perinatal maladaptation, developmental disorders, lung hypoplasia) are closely associated with the epidemiology specific to children, and proposed the new Panama Classification consisting of 10 categories (Table 36). ${ }^{530}$ PPHVD after right-sided heart bypass surgery was allocated as a subcategory of Category 3 (secondary to cardiovascular lesions) in the Panama Classification and was defined as TPG $>6 \mathrm{mmHg}$ or pulmonary vascular resistance index (PVRI) $>3$ Wood Unit $\cdot \mathrm{m}^{2}$, regardless of the $\mathrm{mPAP}$ value.

\subsubsection{Diagnosis}

Transthoracic echocardiography provides a very useful tool for noninvasive determination of complication by pulmonary hypertension or ventricular dysfunction in children with CHD (Recommendation Class I, Evidence Level C), allowing also evaluation of the severity of pulmonary hypertension to some extent (see Section of diagnosis/ evaluation of pulmonary hypertension). Comprehensive differential diagnosis is needed to determine whether the cause of pulmonary hypertension is associated with CHD (Recommendation Class I, Evidence Level C). Also after diagnosis, echocardiography, depending on the condition and changes in treatment, is useful (Recommendation Class I, Evidence Level B), ${ }^{531}$ and its usefulness in combination with other diagnostic imaging modalities, such as heart MRI and CT, has also been reported. ${ }^{332}$

If echocardiography reveals a sign of pressure overload, 


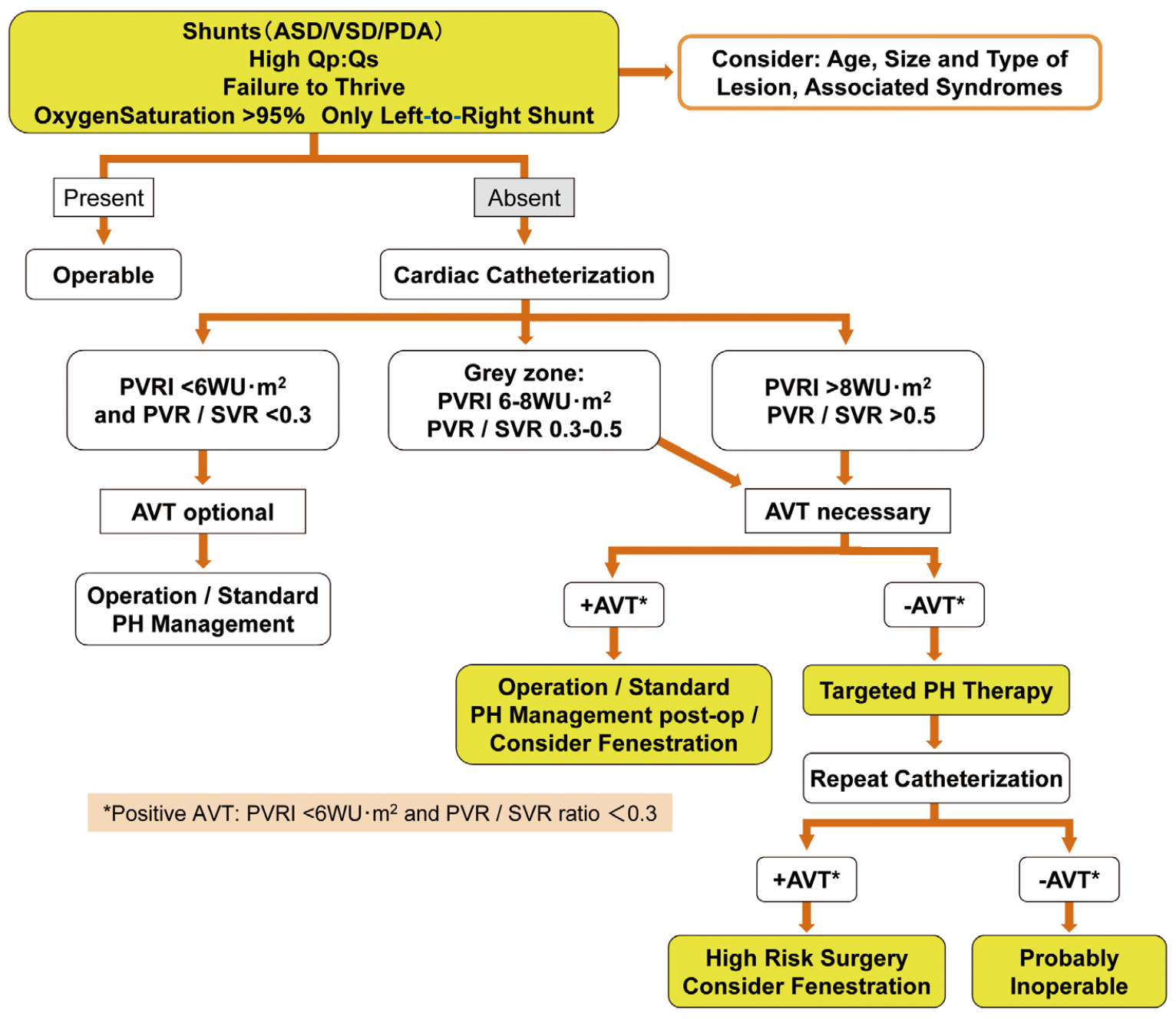

Figure 21. Criteria for surgical repair. (Source: Kozlik-Feldmann R, et al. 2016535)

the presence of pulmonary hypertension is suspected. The most important disease for distinction is disease involving right ventricular overload due to complication by pulmonary artery stenosis. Stenosis in the right ventricle or the main pulmonary artery is easy to distinguish with echocardiography, but it tends to be mistaken as pulmonary hypertension. In infants during the first 3 months after birth, physiological pulmonary hypertension may be present and care needs to be taken to avoid misdiagnosing it as pathologic pulmonary hypertension without sufficient evaluation. Disease involving simple shunt (e.g., septal defect and patent ductus arteriosus) and left ventricular outflow tract obstructive disease (e.g., aortic stenosis) are relatively easy to distinguish with echocardiography, whereas anomalous origin of the right pulmonary artery from the ascending aorta and left ventricular inflow tract obstructive disease (e.g., pulmonary vein stenosis, cor triatriatum, and supravalvular stenosing ring) are difficult to distinguish also with echocardiography and may be mistaken as IPA/HPAH. For this reason, detailed differential diagnosis by not only careful observation by echocardiography but also other diagnostic imaging modalities is required.

For a definite diagnosis of CHD-PAH accompanied by significant left-to-right shunt requires cardiac catheterization before the start of treatment, except for severely sick children (Recommendation Class I, Evidence Level C). ${ }^{531}$ Cardiac catheterization for CHD allows measurement of the pressures such as right atrial pressure and the left atrial pressure, PAWP, calculation of pulmonary blood flow, systemic blood flow (cardiac output), pulmonary/systemic blood flow ratio, and PVRI by measurement of oxygen saturation in the blood sample collected during this procedure, and detailed evaluation of pulmonary hemodynamics. Because the physique differs greatly among individual children, the value of each parameter corrected for the body surface area is usually adopted for the purpose of standardization, and cardiac index (CI) instead of cardiac output and PVRI instead of PVR are used. When converted on the basis of adult body surface area $\left(1.73 \mathrm{~m}^{2}\right)$, PVRI 4 Wood Unit $\cdot \mathrm{m}^{2}$ corresponds to PVR 2.3 Wood Unit, and PVRI 8 Wood Unit $\cdot \mathrm{m}^{2}$ corresponds to PVR 4.6 Wood Unit, for example. PVR $\leq 2-3$ Wood Unit (a normal range according to the guidelines in the USA $)^{\mathbf{5 3 3}}$ corresponds to 


\begin{tabular}{|l|l|}
\hline \multicolumn{2}{|l|}{ Table 37. Criteria for Evaluation of Acute Vasoreactivity Testing (AVT) } \\
\hline Barst Criteria (1986) & mPAP reduced by $\geq 20 \%$ and $\mathrm{Cl}$ unchanged or increased and PVR/SVR reduced or unchanged \\
\hline Rich Criteria (1992) & Both mPAP and PVR reduced by $\geq 20 \%$ \\
\hline Sitbon Criteria (2005) & mPAP reduced by $\geq 10 \%$ and $\mathrm{mPAP} \leq 40 \mathrm{mmHg}$ and CO increased or unchanged \\
\hline PVD Network Criteria (2016) & $\begin{array}{l}\text { (1) IPAH/HPAH: Both mPAP and PVRI/SVRI reduced by }>20 \% \text { and CO unchanged or increased } \\
\text { (2) CHD-PAH with pulmonary/systemic blood flow ratio }>1.5: \text { Both PVRI and PVRI/SVRI reduced by } \\
>20 \% \text { and PVRI }<6 \text { Wood Unit. } \mathrm{m}^{2} \text { and PVRI/SVRI }<0.3\end{array}$ \\
\hline
\end{tabular}

(Source: Prepared based on Rich S, et al. 1992,125 Barst RJ. $1986^{537}$ and Sitbon O, et al. 200353)

Table 38. Recommendation and Evidence Level Concerning Treatment of PPHN

\section{Recommendation}

Oxygen therapy, mechanical ventilation and use of surfactants as needed to maintain preductal $\mathrm{SpO}_{2}$ at 91-95\% for PH management at NICU (Care needs to be taken to avoid lung overswelling, atelectasis, intermittent reduction of oxygen saturation to $\leq 85 \%$ due to pulmonary collapse, and hyperoxia with preductal $\mathrm{SpO}_{2}>97 \%$ )

iNO therapy to improve oxygenation and avoid ECMO during treatment of PPHN in the newborn receiving mechanical ventilation

(a) $\mathrm{PaO}_{2}<100 \mathrm{mmHg}$ during $100 \%$ oxygen therapy

(b) Oxygenation index (OI) $>25$

Setting the target levels of carbon dioxide tension $\left(\mathrm{pCO}_{2}\right) 45-60 \mathrm{mmHg}$, lactate $<5 \mathrm{mmol} / \mathrm{L}$ and $\mathrm{pH}>7.25$ for newborns with acute PPHN immediately after birth (within hours after birth)

Use of prostaglandin $\mathrm{E}_{1}\left(\mathrm{PGE}_{1}\right)$ or $\mathrm{PGE}_{2}$ to keep the ductus arteriosus patent in newborns with right ventricular failure accompanied by severe PPHN and no post-tricuspid-unrestrictive shunt (e.g., without VSD)

Intratracheal surfactant treatment to achieve optimal mechanical ventilation in newborns with PPHN accompanied by impaired pulmonary diffusion function (excluding congenital diaphragmatic hernia) (e.g., newborns with meconium aspiration syndrome accompanied by PPHN)

iNO therapy for PPHN in premature infants (associated with persistent premature rupture of the membrane or oligohydramnion)

Oral sildenafil therapy for PPHN in cases unable to receive iNO therapy

Intravenous prostanoid therapy (via a dedicated central line) or inhalational iloprost therapy for PPHN

Intravenous milrinone therapy for PPHN in patients with left ventricular dysfunction

Treatment of severe PPHN may be extended to ECMO if other intensive care measures fai

Differential diagnosis of lung disease (e.g., ACD and surfactant protein anomaly) in cases of severe PPHN failing to respond to pulmonary vasodilator therapy, pulmonary recruitment and ECMO

ECMO can currently not be recommended for the preterm infant with severe PPHN if gestational age less than 34 weeks and/or body weight less than $2,000 \mathrm{~g}$

\begin{tabular}{|c|c|}
\hline $\begin{array}{c}\text { Recommendation } \\
\text { Class }\end{array}$ & $\begin{array}{c}\text { Evidence } \\
\text { Level }\end{array}$ \\
\hline I & A \\
\hline I & A \\
\hline Ila & B \\
\hline Ila & B \\
\hline Ila & B \\
\hline Ila & B \\
\hline Ila & B \\
\hline Ila & B \\
\hline Ila & B \\
\hline Ila & B \\
\hline Ila & B \\
\hline III & B \\
\hline
\end{tabular}

(Source: Prepared based on Hilgendorff A, et al. 2016557)

PVRI $\leq 1.2-1.7$ Wood Unit $\cdot \mathrm{m}^{2}$ which is approximately consistent with the normal range we experience clinically $\left(1 \leq \mathrm{PVRI} \leq 2 \mathrm{Wood}\right.$ Unit $\left.\cdot \mathrm{m}^{2}\right)$. Furthermore, acute vasoreactivity testing (AVT) ${ }^{\mathbf{5 3 1}}$ and evaluation of dynamic indicators (e.g., response to targeting drug therapy) are also useful.

In Japan, Yamaki et al. devised in 1976 the index of pulmonary vascular disease (IPVD) as an indicator of peripheral pulmonary arteriole pathological findings associated with pulmonary hypertension severity level (an index differing from the Heath-Edwards (HE) Classification) on the basis of observation of the pulmonary vascular lesions during autopsy of patients with complete transposition of the great arteries and ventricular septal defect. ${ }^{67} \mathrm{In}$ 1987, they demonstrated the usefulness of IPVD determined from the biopsied lung tissue in evaluating the indication of radical surgery in patients with atrial septal defect accompanied by severe pulmonary hypertension. ${ }^{69}$ Still, at present, the judgment as to the indications of repair surgery in patients with CHD-PAH accompanied by severe pulmonary hypertension is often based on the pathological findings from the peripheral pulmonary arterioles sampled by lung biopsy. ${ }^{70}$

\subsubsection{Severity and Therapeutic Guidelines}

Because most of the indicators of severity and their values were obtained from evaluation/analysis of children with IPAH, there is no need of using these indicators for evaluation of the severity of PH in children with CHD. However, if any indicator falls under the category of high risk, it is advisable to provide treatment with a goal of reducing the indicator to a range of low risk (a guide for treatment of children with pulmonary hypertension).

For the purpose of preventing pulmonary vascular lesions associated with pulmonary hypertension, it is best to perform surgery at an appropriate timing in cases of repairable CHD. In children with CHD, such as VSD (with considerable amounts of simple left-to-right shunt after the tricuspid valve) or patent ductus arteriosus (PDA), it is necessary to perform repair surgery or palliative surgery (by pulmonary artery banding) within 6 months after birth so that persistence of pulmonary hypertension may be avoided (Recommendation Class I, Evidence Level C) (Table 33). However, care needs to be taken of possible 
onset of pulmonary hypertension crisis during the perioperative period (Recommendation Class IIa, Evidence Level C). In addition, there is a report that $7.4 \%$ of adults with CHD have post-repair surgery PAH. ${ }^{284}$ Still at present, there is no clear-cut consensus view about the criteria for implementation of repair surgery to children with CHDPAH accompanied by PVR elevation. Particularly in cases in which complex cardiac malformation is present, comprehensive evaluation of individual cases is important, including evaluation of the type of underlying heart disease, the location, and amount of shunt, age, disease history, complication, and presence/absence of gene mutation (Recommendation Class I, Evidence Level C). According to the Nice Classification (2013), shunt closure is possible in patients with PVRI $<4$ Wood Unit $\cdot \mathrm{m}^{2}$ and not possible in patients with PVRI $>8 \mathrm{Wood} \mathrm{Unit} \cdot \mathrm{m}^{2}$, whereas patients with $4 \leq \mathrm{PVRI} \leq 8$ Wood Unit $\cdot \mathrm{m}^{2}$ are borderline cases. ${ }^{524}$ Later, an algorithm for treatment of pediatric CHD-PAH incorporating basic variables for evaluation and AVT as a new indictor was published $\mathbf{5 3 4}^{\mathbf{3} 35}$ (Figure 21). According to this algorithm, children with PVRI $<6 \mathrm{Wood} \mathrm{Unit} \cdot \mathrm{m}^{2}$ and PVRI/SVRI $<0.3$ are indicated for repair surgery and closure by intervention if no risk factor for repair surgery is present (Recommendation Class I, Evidence Level C). In patients with PVRI $\geq 6$ Wood Unit $\cdot \mathrm{m}^{2}$ and PVRI/SVRI $\geq 0.3$, evaluation with AVT is needed (Recommendation Class I, Evidence Level C).

For AVT in children with pulmonary hypertension, inhalational nitrogen monoxide, oxygen, inhalational nitrogen monoxide+oxygen, or inhalational iloprost is used. ${ }^{\mathbf{5 3 4}, \mathbf{5 3 6}}$ AVT is not intended to predict long-term prognosis or determine absolute contraindications against repair surgery but is considered to be aimed at predicting short-term or long-term persistence of pulmonary hypertension after surgery. ${ }^{69}$ Criteria for judgment of the outcome of AVT have been proposed by Barst, ${ }^{537}$ Rich $^{125}$ and Sitbon ${ }^{538}$ (Table 37). As the criteria for adult patients, the criteria proposed by Sitbon are now recommended as global guidelines. For pediatric cases, the criteria of Barst are used frequently, but there are both positive and negative views about the validity of these criteria when used for children with $\mathrm{CHD}^{539}$ In 2016, the European Paediatric Pulmonary Vascular Disease (PVD) Network revised the Barst criteria and proposed two different criteria (one for IPAH/HPAH and the other for CHD-PAH) ${ }^{535}$ (Table 37). When the latter criteria is used for shunt disease (pulmonary/systemic blood flow ratio $>1.5$ ), a judgment "positive" is made if both PVRI and PVRI/SVRI decrease to less than $20 \%$ and the requirements PVRI $<6 \mathrm{Wood} \mathrm{Unit} \cdot \mathrm{m}^{2}$ and PVRI/SVRI $<0.3$ are satisfied. Patients with $6 \leq \mathrm{PVRI}<8$ Wood Unit $\cdot \mathrm{m}^{2}$ and $0.3 \leq \mathrm{PVRI} / \mathrm{SVRI} \leq 0.5$ are judged as gray zone cases..$^{\mathbf{5 0 9}, \mathbf{5 3 5}}$

Treatment with targeting drugs before repair surgery in CHD-PAH cases is performed in the gray zone cases mentioned above, for the purpose of improving the condition to a level enabling repair surgery ("treat and repair"). However, there is no sufficient data concerning the longterm prognosis of such a therapeutic strategy, and repair surgery to create fenestration may also be considered in gray zone cases. Most of the past clinical studies on targeting drug therapy for CHD-PAG involved adults with Eisenmenger's syndrome and the efficacy of bosentan was demonstrated in a randomized placebo-controlled study of adults. ${ }^{286}$ It is also possible to use pulmonary hypertensiontargeting drugs alone or in combination to inoperable children with Eisenmenger's syndrome (Recommendation Class IIa, Evidence Level C). A recently reported sub-group analysis of the data from PATENT Study revealed efficacy of riociguat in 35 postoperative children with CHD-PAH. ${ }^{540}$ The results of a global registry study (COMPERA-KIDS) planned to be reported before long are expected to clarify the efficacy of targeting drugs on CHD-PAH.

Regarding the hemodynamics before and after Fontan operation in children with univentricular heart, it is considered favorable to maintain the mean TPG $\leq 6 \mathrm{mmHg}$ as stated in the Section of Pathophysiology (Recommendation Class IIa, Evidence Level C). In children with failing Fontan circulation (TPG $>6 \mathrm{mmHg}$ ), use of pulmonary hypertension-targeting drugs to improve exercise tolerability should be considered (Recommendation Class IIa, Evidence Level C). Furthermore, sildenafil and bosentan have been shown to be effective in improving hemodynamics. ${ }^{541-546}$ In patients after Fontan operation, bosentan has been shown to improve exercise tolerability and oxygen saturation, ${ }^{\mathbf{5 4 7}}$ and sildenafil has been reported to improve exercise tolerability and respiration efficiency. ${ }^{\mathbf{5 4 2}}$ There is no sufficient data about the efficacy of other targeting drugs.

\subsection{Persistent Pulmonary Hypertension of the Newborn (PPHN)}

\section{Summary}

Persistent pulmonary hypertension of the newborn (PPHN) is a condition in which physiological neonatal circulation cannot be established due to failure of PVR reduction after birth, resulting in persistent $\mathrm{PH}$ and hypoxia. This disease is triggered by various factors. A basic strategy for its treatment includes correction of the aggravating factors and implementation of appropriate respiratory/circulatory control, with a goal set at alleviation of hypoxia. Inhaled nitric oxide (iNO) is useful as specific vasodilator therapy. In cases in which iNO is not available, use of phosphodiesterase type 5 (PDE5) inhibitors is considered. There is no established evidence for any other specific pulmonary vasodilator in patients with PPHN. If the disease is therapyresistant, the newborn has been delivered at gestational age 34 weeks or more, the body weight is $2,000 \mathrm{~g}$ or more, and the oxygenation index (OI) remains at 40 or higher, introduction of extracorporeal membrane oxygenation (ECMO) is considered. The recommendation about treatment of PPHN is shown in Table 38.

\section{| 6.3.1 Epidemiology/Prognosis}

The incidence of PPHN has been estimated as 1.9 out of 1,000 births. ${ }^{548}$ This estimation is based on the data from newborns referred to NICU because they required mechanical ventilation or other intensive care. There is a report that early onset of acute PPHN is seen in up to $2 \%$ of all premature infants, with prolonged premature rupture of the membrane and oligohydramnios being risk factors. ${ }^{549}$ Severe PPHN requiring ECMO is more likely to develop in late premature infants and early-term infants than in full-term infants. ${ }^{\mathbf{5 5 0}}$

In the past, when neither NO nor ECMO was used, the mortality rate of patients with PPHN exceeded 50\%. Even after pulmonary vasodilator therapy (with iNO or other means) and ECMO became available with accurate diagnosis, the early mortality rate of patients with PPHN is still as high as $8-10 \%$. It is also known that the incidence of neurodevelopmental impairment (mental retardation, 


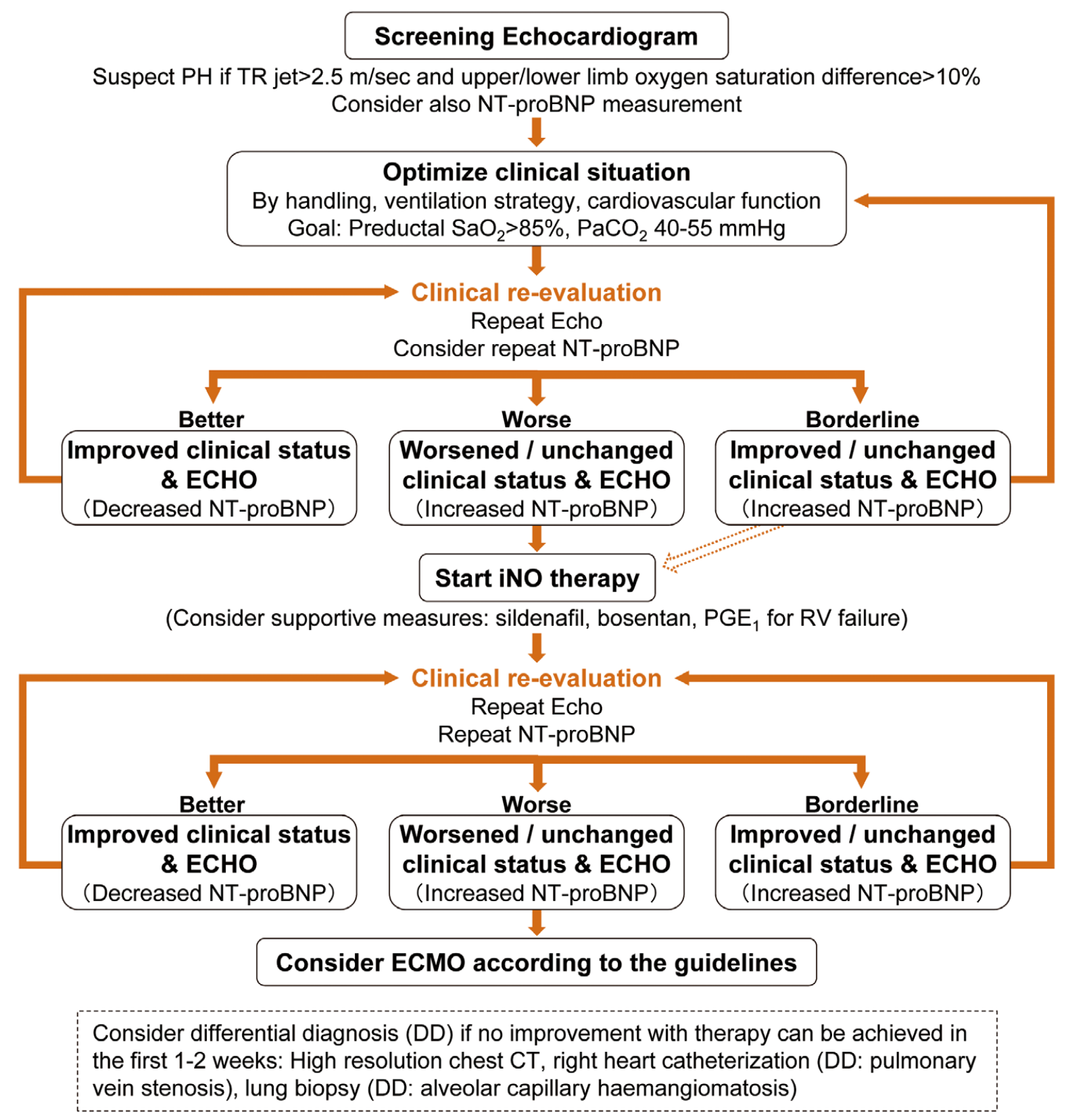

Figure 22. Algorithm for PPHN treatment and management. (Source: Prepared based on Hilgendorff, et al. 2016557)

cerebral palsy, hearing loss, and blindness) is high late after PPHN (at 1 year and 6 months of age). 551

\section{| 6.3.2 Pathophysiology}

If the functional residual capacity of lung is established by the first respiration after birth, PVR begins to decrease sharply, and pulmonary blood flow increases up to 8-10 times that during the intrauterine period, thus establishing postnatal gas exchange. Such reduction in PVR is affected not only by increased oxygen tension and start of respiration but also by the formation of physiological pulmonary vasodilators (nitric oxide [NO] and prostacyclin [PGI 2$]$ ) triggered by blood flow in the lungs. If such postnatal physiological adaptation is impaired by some factors, a pathologic condition (characterized by increases in both PVR and PAP and impaired oxygenation) develops, i.e., a state called "PPHN." PPHN mostly develops immediately or within several days after birth, but a similar pathologic condition occurring within 30 days after birth is also called "PPHN". 530

Known risk factors for onset of PPHN include cesarean section, meconium aspiration syndrome, severe respiratory distress syndrome and sepsis. ${ }^{52}$ PPHN can develop also in the absence of these risk factors, but there are cases in which the condition of CHD is modified by PPHN. There are reports suggesting the association of maternal drug 
intake during the third trimester of pregnancy (e.g., salicylic acid and serotonin reuptake inhibitors) with the onset of PPHN, but there is still discussion about this issue..$^{553,554}$

PPHN often subsides as the underlying disease alleviates in several days after birth. If this condition shows no alleviation within 2 weeks after birth despite appropriate treatment and management and if chest CT scan of such cases indicates lung parenchymal lesions, it is necessary to evaluate lung disorders such as alveolar capillary dysplasia (ACD) or congenital anomalies of surfactant protein. In patients with ACD, mutation of the gene encoding FOX transcription factor at $16 \mathrm{p} 24.1$ has been identified. ${ }^{555}$ At present, there is no established method of treatment for this disease, and patients with this disease often die within 3-6 months. If pulmonary hypertension persists, distinction from pulmonary vein stenosis should also be considered using cardiac catheterization and other modalities of diagnostic imaging (CT and cardiac MRI).

\section{| 6.3.3 Diagnosis and Monitoring}

In cases in which marked hypoxia persists despite appropriate oxygen therapy or mechanical ventilation, resulting in a difference in oxygen saturation between upper and lower extremities, differential diagnosis should be made, including CHD and PPHN as possible diseases. In cases showing greater need of oxygen, profound hypoxia, the need of higher levels of ventilator settings or clinical signs of right ventricular dysfunction, echocardiography should be performed for distinction from PPHN.

In children during the acute stage or recovery stage of pulmonary hypertension, it is advisable to carry out measurement of serum levels of brain natriuretic peptide (BNP) or N-terminal pro-BNP (NT-proBNP) in addition to echocardiography. During management of children with pulmonary hypertension, serum BNP and NT-proBNP levels are repeatedly measured for the purpose of monitoring or as prognosis-related factors. ${ }^{\mathbf{5 5 6}}$ It needs to be considered that these levels can increase also in the presence of systemic blood pressure elevation, PDA, renal dysfunction, or compromised left ventricular function. If pulmonary hypertension persists, despite appropriate treatment of lung disease, it is necessary to carry out evaluation by cardiac catheterization before the start of pulmonary vasodilator treatment unless there is no clinical disadvantage expected from cardiac catheterization.

\subsubsection{General Treatment for PPHN in Term Infants and Premature Infants}

Figure 22 illustrates the algorithm for treatment and management of PPHN ${ }^{557}$ and Table 38 shows the recommendation of treatment for PPHN. ${ }^{\mathbf{5 0 8 , 5 5 7}}$

First, it is essential to minimize the PPHN-aggravating environmental factors (manual manipulation, tracheal aspiration, heel prick test).

A basic principle of respiration and circulation management in patients with PPHN is to maintain appropriate lung recruitment, alveolar ventilation, oxygen supply, and cardiac function. During mechanical ventilation, care needs to be taken to prevent pulmonary barotrauma and volutrauma by avoiding overinflation of lungs and high mean airway pressure (MAP). Adjustment should be made to keep preductal arterial oxygen saturation $\left(\mathrm{SaO}_{2}\right)$ at $85-93 \%$ and arterial carbon dioxide tension $\left(\mathrm{PaCO}_{2}\right)$ at 40-55 mmHg. ${ }^{558}$ High-concentration oxygen is often used, but it needs to be considered that adverse influence, such as lung damage, can arise if the inhaled oxygen concentration exceeds $60 \%\left(\mathrm{FiO}_{2}>0.6\right)$. If lungs cannot be sufficiently inflated, high-frequency ventilation is used, but care needs to be taken to avoid overinflation. In cases of PPHN due to meconium aspiration syndrome, administration of an artificial surfactant may improve alveolar ventilation, leading to improved condition and avoidance of ECMO. ${ }^{559}$ However, attention is needed to the possibility that acute airway obstruction can be caused by administration of an artificial surfactant.

Acidosis can cause pulmonary vasoconstriction and therefore requires treatment. To keep the blood gas $\mathrm{pH}$ 7.45-7.55 to control PVR, sodium bicarbonate is sometimes administered repeatedly, but care needs to be taken of adverse effects. Intentional induction of alkalosis by hyperventilation is sometimes used, but the long-term prognosis after this procedure is unknown.

Efforts should be made to keep systemic blood pressure in the physiological range for the age (gestational weeks) and to adjust the intravenous fluid volume and control cardiovascular agonists so that cardiac output can be preserved. Excessive increased blood pressure should be avoided because it cannot lower PVR although it appears to temporarily improve oxygenation.

Polycythemia needs to be corrected because it can cause increased PVR by increasing blood viscosity.

Simultaneously with the general treatment mentioned above, it is advisable to consider introduction of pulmonary vasodilator therapy (iNO, pulmonary vasodilators) or ECMO in PPHN presenting with persistent hypoxia and unstable hemodynamics.

\subsection{5 iNO for PPHN}

Infants with PPHN presenting with significant right-toleft-shunt require pulmonary vasodilator therapy.

Because iNO has been shown to reduce the incidence of composite endpoint (death or ECMO introduction) and to improve the prognosis, ${ }^{\mathbf{5 6 0}} \mathrm{iNO}$ is first recommended as a means of specific pulmonary vasodilator therapy. About half of all PPHN patients with significant right-to-left shunt respond to iNO, resulting in improved oxygenation. Usually, 5-20 ppm iNO is used, at the initial concentration of $20 \mathrm{ppm}$ in many cases. If its concentration exceeds $20 \mathrm{ppm}$, iNO does not further improve oxygenation or prognosis but can increase the risk for methemoglobinemia and other complications. In premature infants with respiratory disorders, routine use of iNO as a means of preventing the development of acute or chronic pulmonary hypertension is not always recommended.561 In patients with congenital diaphragmatic hernia $(\mathrm{CDH})$, use of iNO as a bridge to introduction of ECMO (oxygen index: $\mathrm{FiO}_{2} \times 100 \times$ MAP/arterial oxygen tension $\left[\mathrm{PaO}_{2}\right]>20$, discrepancy in oxygen saturation between upper and lower extremities $>10 \%$ ) is sometimes considered, but there is no evidence for its effectiveness. ${ }^{\mathbf{5 6 2}}$

There is no optimal procedure for iNO weaning. Once improvement of oxygenation has been achieved, the iNO dose level can be usually reduced relatively rapidly to $5 \mathrm{ppm}$ and the use of iNO can be often discontinued within 5 days. ${ }^{563}$ After discontinuation of iNO, rebounding of PPHN is sometimes seen. In such cases, the iNO dose reduction procedure should be improved, e.g., by reducing the iNO dose level to $1 \mathrm{ppm}$ before use of iNO is discontinued. If PPHN fails to show alleviation despite 5-day or longer treatment, distinction from underlying disease, such 
as ACD, is needed.

\subsubsection{ECMO for PPHN}

ECMO is introduced for the purpose of alleviating rightsided heart failure by maintaining effective oxygenation and carbon dioxide elimination and reducing pulmonary barotrauma and volutrauma. The criteria for introduction of ECMO are given below. ${ }^{\mathbf{5 6 4}}$

- Preductal $\mathrm{SpO}_{2}<85 \%$ even when peak inspiratory pressure $(\mathrm{PIP})>28 \mathrm{cmH}_{2} \mathrm{O}\left(\mathrm{MAP}>15 \mathrm{cmH}_{2} \mathrm{O}\right.$ during high frequency oscillation [HFO])

- PPHN and circulatory failure resistant to appropriate treatment

- Gestational age $>34$ weeks

- Body weight $>2 \mathrm{~kg}$

As additional criteria, consistent OI $\geq 40$, hypotension resistant to water load and inotropic therapy, 12-24 hour urine volume $<0.05 \mathrm{~mL} / \mathrm{kg} /$ hour, and metabolic acidosis (lactate $>5 \mathrm{mmol} / \mathrm{L}, \mathrm{pH}<7.15$ ) have also been listed.

\section{6.3.7 Drugs for Treatment of PPHN}

In cases of PPHN, the experience using pulmonary hypertension targeting drugs available at present is limited. If pulmonary hypertension targeting drugs with systemic effect are used, care needs to be taken of possible aggravation of hypoxia (due to ventilation-perfusion imbalance) and onset of hypotension.

Because activation of PDE5 contributes to the pathophysiology of PPHN, effectiveness of PDE5 inhibitors, such as sildenafil, is suggested. In practice, tolerability of sildenafil in newborns with PPHN has been demonstrated, and this drug has been used by administration via a feeding tube in a starting dose of $0.5-1.0 \mathrm{mg} / \mathrm{kg} / \mathrm{dose}$ every 6 hours. ${ }^{565}$ Meta-analysis of the data from 3 clinical studies (involving 77 newborns with PPHN in total) demonstrated the possibility of sildenafil to improve oxygenation and reduce the mortality rate. ${ }^{566}$ Use of sildenafil for treatment of PPHN should be considered particularly when iNO is not available.

$\mathrm{PGI}_{2}$ and its derivatives are also used, but only reports in a small number of cases or on short-term use are available. There is a report that inhaled prostacyclin can improve oxygenation in iNO-resistant cases of PPHN. ${ }^{\mathbf{5 6 7}}$

In newborns with PPHN, blood endothelin-1 level is high. ${ }^{568}$ Although there are case reports of alleviation of $\mathrm{PPHN}$ in response to bosentan treatment, evidence is not sufficient to allow extensive recommendation of this therapy. ${ }^{569}$ The results of FUTURE4 Study (bosentan treatment in PPHN cases) were negative, revealing a problem with absorption of bosentan via the intestine. ${ }^{570}$

In cases accompanied by right ventricular dysfunction, attempts of reducing the right ventricular load by using PGE1 or maintaining the ductus arteriosus patent may be considered. However, such measures will reduce oxygen saturation in the lower extremities.

There are many open questions about the influence of each treatment for PPHN on the long-term prognosis. Prospective comparative studies will be needed.

\section{Definition of Pulmonary Hypertension Expert Referral Center}

In Japan, the organization named "Pulmonary Hypertension Expert Referral Centre" has already been founded at several places, but the organization does not seem to have reached a level of functioning comparable to that of similar centers in Western countries. The ESC/ERS Guidelines for the Diagnosis and Treatment of Pulmonary Hypertension $2015^{1}$ proposed to use the expression "pulmonary hypertension referral center" for qualified organization. To improve the outcome in pulmonary hypertension patients and from the medico-economic viewpoint, establishment of a group of experts in pulmonary hypertension has been necessary and, pulmonary hypertension referral centers have been established. Such movements have been supported by patient organizations as well. The purpose of establishing the pulmonary hypertension referral center is to accept all new patients with pulmonary hypertension, regardless of the etiological type, for providing evaluation and appropriate treatment specific to pulmonary hypertension. It is additionally aimed at facilitating routine sharing of information with affiliated healthcare professionals and promoting research and education in addition to clinical care.

The definition of pulmonary hypertension referral center proposed in the ESC/ERS Guidelines for the Diagnosis and Treatment of Pulmonary Hypertension 2015 is given below. The proposed definition covers what needs to be considered as goals, although it is an open question whether such goals fit the current situations in Japanese healthcare.

Pulmonary hypertension expert referral centers are:

1. It is recommended for expert referral centers to provide care by a multiprofessional team (cardiology and respiratory medicine physicians, clinical nurse specialist, radiologists, psychological and social work support, appropriate on-call expertise).

2. It is recommended for expert referral centers to have direct links and quick referral patterns to other services (such as CTD treatment, family planning, PEA, lung transplantation, adult congenital heart disease treatment).

3. It should be considered that an expert referral center follows at least 50 patients with PAH or CTEPH and should receive at least two new referrals per month with documented PAH or CTEPH.

4. It should be considered that an expert referral center performs at least 20 vasoreactivity tests in idiopathic PAH, drug-induced PAH or heritable PAH per year.

5. Expert referral centers should participate in collaborative clinical research in PAH, including phase II and phase III clinical trials. 


\section{Pulmonary Hypertension Under the Measures Against Intractable Diseases}

As for the diseases responsible for pulmonary hypertension, pulmonary arterial hypertension (No. 86), chronic thromboembolic pulmonary hypertension (No. 88), and pulmonary veno-occlusive disease/pulmonary capillary hemangiomatosis (No. 87) are listed as intractable diseases according to the measures against intractable diseases. Patients with these diseases can receive public subsidy to healthcare expenses (listed intractable disease program) if the diagnosis is based in accordance with the official guidance and an application for the benefits is filed.
Details are given on the website of the Japan Intractable Diseases Information Center (http://www.nanbyou.or.jp) (information source: Respiratory Disease Investigation/ Study Group [Intractable respiratory disease/pulmonary hypertension]).

The outline of this disease and part of its diagnostic criteria are presented below on the basis of the documents concerning the intractable diseases listed by the measures against intractable diseases (enforced on January 1, 2015) (Document 1,571 Document 2,572 Document $3^{573}$ ). ${ }^{1}$ 


\section{Document 1 Pulmonary Arterial Hypertension (Listed Intractable Disease No. 86)}

\section{$<$ Diagnostic Criteria $>$}

Before pulmonary arterial hypertension is diagnosed, it is necessary to judge the association of pulmonary hypertension with the pulmonary artery by right heart catheterization, to make differential diagnosis based on clinical classification and to rule out other diseases that can cause pulmonary hypertension.

\section{(1) Test Findings}

1) Right heart catheterization

(a) Elevation in pulmonary artery pressure (mean pulmonary artery pressure at rest $\geq 25 \mathrm{mmHg}$, pulmonary vascular resistance $\geq 3$ Wood Unit, 240 dyne $\left.\cdot \mathrm{sec} \cdot \mathrm{cm}^{-5}\right)$

(b) Pulmonary artery wedge pressure (left atrial pressure) normal $(\leq 15 \mathrm{mmHg})$

2) No segmental perfusion defect revealed by pulmonary perfusion scintigraphy (normal or signs of spotted perfusion defect in cases of idiopathic or hereditary pulmonary arterial hypertension).

(2) Reference Test Findings

1) Echocardiographic findings of tricuspid systolic pressure gradient $\geq 40 \mathrm{mmHg}$, marked increase in estimated pulmonary artery pressure, and right ventricular dilatation

2) Chest X-ray findings of pulmonary artery trunk dilatation and peripheral pulmonary vessel shadow narrowing

3) Electrocardiographic finding of right ventricular hypertrophy

(3) Major Symptoms and Clinical Findings

1) Shortness of breath on exertion

2) Tendency to fatigue

3) Syncope

4) Auscultation signs of pulmonary hypertension (e.g., enhanced pulmonary artery element of second heart sound)

(4) Clinical Classification of Pulmonary Arterial Hypertension

Differential diagnosis is made to determine the type of pulmonary arterial hypertension for which the following types are known.

1) Idiopathic or heritable pulmonary arterial hypertension

2) Pulmonary arterial hypertension associated with collagen disease

3) Pulmonary arterial hypertension associated with congenital shunt-involving heart disease

4) Pulmonary arterial hypertension associated with portal hypertension

5) Pulmonary arterial hypertension associated with HIV infection

6) Drug-induced pulmonary arterial hypertension

7) Pulmonary arterial hypertension accompanying a respiratory disease

However, the diagnosis of pulmonary arterial hypertension associated with congenital shunt-involving heart disease is limited to inoperable cases and cases presenting with pulmonary arterial hypertension persisting after operation. In cases in which this diagnosis is made, test data/findings (e.g., echocardiographic findings, findings from diagnostic imaging such as chest X-ray and CT) should be attached.

\section{(5) Ruling Out of the Diseases Possibly Causing Pulmonary Hypertension}

The diseases listed below differ in pathophysiology from pulmonary arterial hypertension but can cause pulmonary hypertension, possibly leading to right ventricular hypertrophy. These diseases need to be ruled out.

1) Pulmonary hypertension due to left heart disease

2) Pulmonary hypertension due to respiratory diseases and/or hypoxia

3) Chronic thromboembolic pulmonary hypertension

4) Pulmonary veno-occlusive disease/pulmonary capillary disease

5) Other types of pulmonary hypertension (e.g., secondary pulmonary hypertension due to sarcoidosis, Langerhans cell histiocytosis, lymphangiomyomatosis, aortitis syndrome, congenital pulmonary vascular anomaly, primary pulmonary artery sarcoma, and external compression of pulmonary vessels)

Among cases of pulmonary hypertension due to respiratory diseases and/or hypoxia, there are cases presenting with marked pulmonary hypertension that cannot be explained by respiratory diseases and/or hypoxia alone. In such cases, it is acceptable to diagnosis complication by pulmonary arterial hypertension. Attachment of test data/findings (e.g., echocardiographic finding, findings from diagnostic imaging such as chest X-ray and CT, and respiratory function test data) is needed in such cases.

\section{(6) Criteria for Official Registration}

All of the following requirements need to be satisfied.

1) Satisfaction of the requirements as to test findings for diagnosis (findings from right heart catheterization and pulmonary perfusion scintigraphy)

2) Can rule out all diseases listed as diseases to be ruled out

3) Falling under any of the clinical types 1) to 7) of pulmonary arterial hypertension

\section{<Severity Rating>}

Stage 3 or advanced cases are covered. 


\section{(New Registration)}

\begin{tabular}{|c|c|c|c|c|}
\hline $\begin{array}{l}\text { Upon application } \\
\text { for registration }\end{array}$ & Subjective symptoms & $\begin{array}{l}\text { Mean pulmonary artery } \\
\text { pressure (mPAP) }\end{array}$ & Cardiac index (CI) & Use of pulmonary vasodilators \\
\hline Stage 1 & $\begin{array}{l}\text { NYHA/WHO functional } \\
\text { class I/II }\end{array}$ & $40>\mathrm{mPAP} \geq 25 \mathrm{mmHg}$ & & Not used \\
\hline Stage 2 & $\begin{array}{l}\text { NYHA/WHO functional } \\
\text { class I/II }\end{array}$ & $\mathrm{mPAP} \geq 40 \mathrm{mmHg}$ & & Not used \\
\hline \multirow[t]{2}{*}{$\overline{\text { Stage }} \overline{3}$} & $\begin{array}{l}\text { NY} \overline{\mathrm{H}} \overline{\mathrm{A}} / \overline{\mathrm{W}} \overline{\mathrm{H} O} \text { functional } \\
\text { class I/II }\end{array}$ & $\overline{\mathrm{mPA}} \overline{\mathrm{P}} \geq \overline{25 \mathrm{~mm}} \overline{\mathrm{Hg}}$ & & 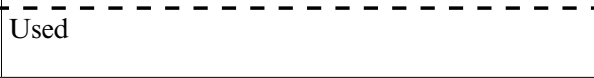 \\
\hline & $\begin{array}{l}\text { NYHA/WHO functional } \\
\text { class III/IV }\end{array}$ & $\mathrm{mPAP} \geq 25 \mathrm{mmHg}$ & $\mathrm{CI} \geq 2.5 \mathrm{~L} / \mathrm{min} / \mathrm{m}^{2}$ & Regardless of use/non-use \\
\hline Stage 4 & $\begin{array}{l}\text { NYHA/WHO functional } \\
\text { class III/IV }\end{array}$ & $\mathrm{mPAP} \geq 25 \mathrm{mmHg}$ & $\mathrm{CI}<2.5 \mathrm{~L} / \mathrm{min} / \mathrm{m}^{2}$ & Regardless of use/non-use \\
\hline \multirow[t]{2}{*}{ Stage 5} & $\begin{array}{l}\text { NYHA/WHO functional } \\
\text { class IV }\end{array}$ & $\mathrm{mPAP} \geq 40 \mathrm{mmHg}$ & & Regardless of use/non-use \\
\hline & & & & $\begin{array}{l}\text { Stage 5, regardless of subjective symptom severity } \\
\text { and mPAP, if PGI } 2 \text { serial intravenous infusion/ } \\
\text { continued subcutaneous injection is needed }\end{array}$ \\
\hline
\end{tabular}

The highest stage satisfying all subjective symptoms, mPAP, CI, and use of pulmonary vasodilators is selected.

(Renewal of Registration)

\begin{tabular}{|c|c|c|c|}
\hline Upon renewal & Subjective symptoms & $\begin{array}{l}\text { Tricuspid regurgitation pressure gradient } \\
\text { measured (TRPG) by echocardiography }\end{array}$ & Use of pulmonary vasodilators \\
\hline Stage 1 & $\begin{array}{l}\text { NYHA/WHO functional } \\
\text { class I/II }\end{array}$ & $\begin{array}{l}\text { TRPG }<40 \mathrm{mmHg} \\
\text { or no significant TR }\end{array}$ & Not used \\
\hline \multirow[t]{2}{*}{ Stage 2} & $\begin{array}{l}\text { NYHA/WHO functional } \\
\text { class I/II }\end{array}$ & TRPG $\geq 40 \mathrm{mmHg}$ & Not used \\
\hline & $\begin{array}{l}\text { NYHA/WHO functional } \\
\text { class I }\end{array}$ & $\begin{array}{l}\text { TRPG }<40 \mathrm{mmHg} \\
\text { or no significant TR }\end{array}$ & Used \\
\hline \multirow[t]{3}{*}{$\overline{\text { Stage }} \overline{3}$} & $\begin{array}{l}\overline{\mathrm{NY}} \overline{\mathrm{H}} \overline{\mathrm{A}} / \overline{\mathrm{W}} \overline{\mathrm{H} O} \overline{\mathrm{functional}} \\
\text { class I/II }\end{array}$ & $\overline{\mathrm{TR}} \overline{\mathrm{P} G} \geq \overline{4} 0 \overline{\mathrm{mm}} \overline{\mathrm{Hg}}$ & Used ----- \\
\hline & $\begin{array}{l}\text { NYHA/WHO functional } \\
\text { class III }\end{array}$ & TRPG $\geq 40 \mathrm{mmHg}$ & Not used \\
\hline & $\begin{array}{l}\text { NYHA/WHO functional } \\
\text { class II/III }\end{array}$ & TRPG $<40 \mathrm{mmHg}$ & Used \\
\hline \multirow[t]{2}{*}{ Stage 4} & $\begin{array}{l}\text { NYHA/WHO functional } \\
\text { class II/III }\end{array}$ & TRPG $\geq 60 \mathrm{mmHg}$ & Regardless of use/non-use \\
\hline & $\begin{array}{l}\text { NYHA/WHO functional } \\
\text { class IV }\end{array}$ & TRPG $<60 \mathrm{mmHg}$ & Regardless of use/non-use \\
\hline \multirow[t]{2}{*}{ Stage 5} & $\begin{array}{l}\text { NYHA/WHO functional } \\
\text { class IV }\end{array}$ & TRPG $\geq 60 \mathrm{mmHg}$ & Regardless of use/non-use \\
\hline & & & $\begin{array}{l}\text { Stage 5, regardless of WHO-PH classification } \\
\text { and mPAP, if PGI } 2 \text { serial intravenous infusion/ } \\
\text { continued subcutaneous injection is needed }\end{array}$ \\
\hline
\end{tabular}

The highest stage satisfying all subjective symptoms, TRPG, and use of pulmonary vasodilators is selected.

(Reference information)

- In Stage 3 or more advanced cases, it is desirable to perform evaluation by cardiac catheterization at least once in 2 years. However, in patients at elevated risk involved in cardiac catheterization (e.g., children, elderly patients, and patients with multiple accompanying diseases), it is acceptable to perform echocardiographic evaluation depending on the conditions of individual cases.

- TRPG $40 \mathrm{mmHg}$ is equivalent to $\mathrm{mPAP} 25 \mathrm{mmHg}$. TRPG $60 \mathrm{mmHg}$ is equivalent to $\mathrm{mPAP} 40 \mathrm{mmHg}$.

*Precautions in performing the diagnostic criteria and severity rating

1. If the diagnostic criteria include no particular provision about the clinical symptoms, test findings to be used for disease diagnosis, the symptoms/findings obtained at any point of time may be used (although limited to clinical symptoms presenting the course of the illness concerned and possible to be confirmed).

2. In cases in which the severity rating is done after the start of treatment, the worst condition during the latest 6 months observed during treatment under appropriate medical management should be judged by the physician.

3. The public healthcare expense subsidy program also covers patients whose severity level does not fall under the preset level of the above-mentioned severity classification if continuation of high-cost clinical care is needed. 


\section{Document 2 Chronic Thromboembolic Pulmonary Hypertension (Listed Intractable Disease No. 88)}

\section{$<$ Diagnostic Criteria $>$}

Chronic thromboembolic pulmonary hypertension involves chronic obstruction of the pulmonary artery due to organized thrombus, resulting in pulmonary hypertension and manifestation of dyspnea on exertion and other clinical symptoms. Before diagnosis of this disease, it is necessary to diagnose pulmonary hypertension by right heart catheterization and to rule out other diseases that can cause pulmonary hypertension.

\section{(1) Test Findings}

1) Right heart catheterization

1. Increased pulmonary artery pressure (mean pulmonary artery pressure at rest $\geq 25 \mathrm{mmHg}$ )

2. Pulmonary artery wedge pressure (right atrial pressure) normal $(\leq 15 \mathrm{mmHg})$

2) Ventilation/perfusion lung scan

Segmental perfusion defects (segmental defects), not accompanied by abnormal ventilation, remain unchanged or are estimated to remain unchanged for 6 months or more after thrombolytic therapy or anticoagulant therapy. In cases "estimated," a check of unchanged segmental defects is needed 6 months after the therapy.

3) Pulmonary angiography

At least one of 5 changes (1. pouch defects, 2. webs and bands, 3.intimal irregularities, 4. abrupt narrowing, 5. complete obstruction) is demonstrated as a change caused by chronic thrombosis.

4) Contrast-enhanced chest $\mathrm{CT}$

Contrast-enhanced CT reveals at least one of the 5 changes (1. mural defects, 2. webs and bands, 3. intimal irregularities, 4. abrupt narrowing, 5. complete obstruction) as a change caused by chronic thrombosis.

(2) Test Findings Valuable as Reference Information

1) Echocardiography

1. Right ventricular dilatation and septum flattening

2. Patterns characteristic of pulmonary hypertension or signs of high right ventricular systolic pressure (tricuspid regurgitation pressure gradient $\geq 40 \mathrm{mmHg}$ ) revealed by Doppler echocardiography

3. Reduction of tricuspid annual plane systolic excursion (TAPSE)

2) Arterial blood gas data

1. Hypoxia accompanied by hypocapnia $\left(\mathrm{PaCO}_{2} \leq 35\right.$ Torr, $\mathrm{PaO}_{2} \leq 70$ Torr $)$

2. $\mathrm{AaDO}_{2}$ enlargement $\left(\mathrm{AaDO}_{2} \geq 30\right.$ Torr $)$

3) Chest X-ray

1. Enlargement of pulmonary artery shadow at pulmonary hilum (protrusion of left second arch or dilatation of right pulmonary artery's descending branch: maximum diameter $\geq 18 \mathrm{~mm}$ )

2. Enlargement of cardiac shadow $(\mathrm{CTR} \geq 50 \%)$

3. Local difference in lung field vascular shadow (between right/left or upper/lower lung fields)

4) Electrocardiography

1. Right axis deviation and right atrium load

2. $\mathrm{R} \geq 5 \mathrm{~mm}$ or $\mathrm{R} / \mathrm{S}>1$ with $\mathrm{V} 1, \mathrm{~S} \geq 7 \mathrm{~mm}$ or $\mathrm{R} / \mathrm{S} \leq 1$ with $\mathrm{V} 5$

(3) Major Symptoms and Clinical Findings

1) Shortness of breath on exertion

2) Clinical symptoms known of acute cases (e.g., sudden dyspnea, chest pain, and syncope), previously seen at least once

3) Clinical symptoms suggesting deep vein thrombosis of legs (leg swelling and pain), previously seen at least once

4) Pulmonary vascular murmur present in the lung field

5) Abnormality suggesting pulmonary hypertension (at least one of IIp (II) sound enhancement, III/VI sound, pulmonary valve regurgitation sound, and tricuspid regurgitation sound) revealed by chest auscultation

\section{(4) Diseases to be Ruled Out}

The following conditions, other than chronic thromboembolic pulmonary hypertension, need to be ruled out because they can also cause pulmonary hypertension possibly leading to right ventricular hypertrophy and chronic cor pulmonale.

1. Idiopathic or heritable pulmonary arterial hypertension

2. Pulmonary arterial hypertension associated with collagen disease

3. Pulmonary arterial hypertension associated with shunt-involving heart disease

4. Pulmonary arterial hypertension associated with portal hypertension

5. Pulmonary arterial hypertension associated with HIV infection

6. Drug/toxin-induced pulmonary arterial hypertension

7. Pulmonary veno-occlusive disease, pulmonary capillary hemangiomatosis

8. Persistent pulmonary hypertension in the newborn

9. Pulmonary hypertension due to left heart disease

10. Pulmonary hypertension due to respiratory diseases and/or hypoxia

11. Other types of pulmonary hypertension (secondary pulmonary hypertension due to sarcoidosis, Langerhans cell histiocytosis, lymphangiomyomatosis, aortitis syndrome, congenital pulmonary vascular anomaly, primary pulmonary artery sarcoma, and external compression of pulmonary vessels)

(5) Criteria for Official Registration

All of the following requirements need to be satisfied.

1) Application for New Registration

1. Satisfying the right heart catheterization findings listed in the test findings for diagnosis 
2. Satisfying the ventilation/perfusion lung scan findings listed in the test findings for diagnosis

3. Satisfying the pulmonary angiography and/or contrast-enhanced chest CT findings listed in the test findings for diagnosis

4. Able to rule out all diseases that need to be ruled out

5. *Describing the month planned for surgery or BPA (PTPA) in cases in which such a procedure is planned

2) Application for Renewal of Registration

Cases are roughly divided into two groups, i.e., the group of operated cases and the group of cases having undergone BPA (PTPA).

1. Operated cases and cases having undergone BPA (PTPA)

a) Describing the date of operation or first session of BPA

b) Presenting any of the ventilation/perfusion lung scan findings, contrast-enhanced chest CT findings, or pulmonary angiography findings listed in the test findings for diagnosis (indispensable if the severity level is elevated from the previously registered level)

c) Satisfying the right heart catheterization findings or the echocardiography findings (listed as the reference test findings)

d) Able to rule out all diseases that need to be ruled out.

2. Non-operated cases

Cases requiring continuation of treatment although pulmonary hypertension has become less mild or returned to normal as compared to the condition upon application for new registration thanks to treatment (e.g., pulmonary vasodilator therapy with riociguat).

a) Presenting any of the ventilation/perfusion lung scan findings, contrast-enhanced chest CT findings, or pulmonary angiography findings listed in the test findings for diagnosis (indispensable if the severity level is elevated from the previously registered level)

b) Satisfying the right heart catheterization findings or the echocardiography findings (listed as the reference test findings)

c) Able to rule out all diseases that need to be ruled out.

\section{$<$ Severity Rating >}

Official registration is accepted in Stage 2 or advanced cases rated in accordance with the Study Group's severity classification based on NYHA Cardiac Function Classification and WHO Pulmonary Hypertension Functional Classification.

\section{(New Registration)}

\begin{tabular}{|c|c|c|c|c|c|}
\hline $\begin{array}{l}\text { Upon application } \\
\text { for registration }\end{array}$ & Subjective symptoms & $\begin{array}{l}\text { Mean pulmonary artery } \\
\text { pressure (mPAP) }\end{array}$ & $\begin{array}{l}\text { Pulmonary vascular } \\
\text { resistance (PVR) }\end{array}$ & $\begin{array}{l}\text { Resting/indoor } \\
\text { air } \mathrm{PaO}_{2} \text { (Torr) }\end{array}$ & $\begin{array}{l}\text { Use of pulmonary } \\
\text { vasodilators }\end{array}$ \\
\hline Stage 1 & $\begin{array}{l}\text { NYHA/WHO functional } \\
\text { class I }\end{array}$ & $\mathrm{mPAP} \geq 25 \mathrm{mmHg}$ & & & Regardless of use/non-use \\
\hline$\overline{\text { Stage }} \overline{2}-$ & $\begin{array}{l}\mathrm{NY} \overline{\mathrm{H}} \overline{\mathrm{A}} / \overline{\mathrm{W}} \overline{\mathrm{H}} \overline{\mathrm{O}} \text { functional } \\
\text { class II }\end{array}$ & $\overline{\mathrm{mPA}} \overline{\mathrm{P}} \geq \overline{25} \overline{\mathrm{mm}} \overline{\mathrm{mg}}$ & & $\overline{\mathrm{PaO}_{2}} \overline{\mathbf{7}} \overline{70} \overline{\text { torr }}$ & Regardless of use/non-use \\
\hline \multirow[t]{3}{*}{ Stage 3} & $\begin{array}{l}\text { NYHA/WHO functional } \\
\text { class II }\end{array}$ & $\mathrm{mPAP} \geq 25 \mathrm{mmHg}$ & & $\mathrm{PaO}_{2}<70$ torr & Regardless of use/non-use \\
\hline & $\begin{array}{l}\text { NYHA/WHO functional } \\
\text { class II }\end{array}$ & $\mathrm{mPAP} \geq 25 \mathrm{mmHg}$ & & & Used \\
\hline & $\begin{array}{l}\text { NYHA/WHO functional } \\
\text { class III/IV }\end{array}$ & $\mathrm{mPAP} \geq 25 \mathrm{mmHg}$ & & & Regardless of use/non-use \\
\hline Stage 4 & $\begin{array}{l}\text { NYHA/WHO functional } \\
\text { class III/IV }\end{array}$ & $\mathrm{mPAP} \geq 30 \mathrm{mmHg}$ & & & Regardless of use/non-use \\
\hline Stage 5 & $\begin{array}{l}\text { NYHA/WHO functional } \\
\text { class I/II/III/IV }\end{array}$ & & $\begin{array}{l}\mathrm{PVR} \geq 1,000 \text { dyne } \cdot \mathrm{s} \cdot \mathrm{cm}^{-5} \\
(12.5 \text { Wood Unit) }\end{array}$ & & Regardless of use/non-use \\
\hline
\end{tabular}

The highest stage satisfying all subjective symptoms, mPAP, PVR, resting/indoor air $\mathrm{PaO}_{2}$, and use of pulmonary vasodilators is selected. 
(Renewal of Registration)

\begin{tabular}{|c|c|c|c|c|}
\hline $\begin{array}{l}\text { Upon } \\
\text { renewal }\end{array}$ & Subjective symptoms & $\begin{array}{l}\text { Tricuspid regurgitation pressure } \\
\text { gradient (TRPG) measured by } \\
\text { echocardiography }\end{array}$ & $\begin{array}{l}\text { Mean pulmonary artery pressure } \\
\text { (mPAP) and pulmonary vascular } \\
\text { resistance (PVR) during right } \\
\text { heart catheterization }\end{array}$ & $\begin{array}{l}\text { Use of pulmonary } \\
\text { vasodilators or HOT }\end{array}$ \\
\hline Stage 1 & $\begin{array}{l}\text { NYHA/WHO functional } \\
\text { class I }\end{array}$ & & & \\
\hline$\overline{\text { Stage }} \overline{2}$ & 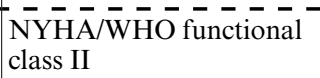 & $1-$ & - & Regardless of use/non-use \\
\hline \multirow[t]{2}{*}{ Stage 3} & $\begin{array}{l}\text { NYHA/WHO functional } \\
\text { class II/III/IV }\end{array}$ & TRPG $<40 \mathrm{mmHg}$ & $\mathrm{mPAP}<25 \mathrm{mmHg}$ & Used \\
\hline & $\begin{array}{l}\text { NYHA/WHO functional } \\
\text { class II }\end{array}$ & TRPG $\geq 40 \mathrm{mmHg}$ & $\mathrm{mPAP} \geq 25 \mathrm{mmHg}$ & Regardless of use/non-use \\
\hline Stage 4 & $\begin{array}{l}\text { NYHA/WHO functional } \\
\text { class III/IV }\end{array}$ & TRPG $\geq 40 \mathrm{mmHg}$ & $\mathrm{mPAP} \geq 25 \mathrm{mmHg}$ & Regardless of use/non-use \\
\hline \multirow[t]{2}{*}{ Stage 5} & $\begin{array}{l}\text { NYHA/WHO functional } \\
\text { class I/II/III/IV }\end{array}$ & & $\begin{array}{l}\text { PVR } \geq 1,000 \text { dyne } \cdot \mathrm{s} \cdot \mathrm{cm}^{-5} \\
\text { (12.5 Wood Unit) }\end{array}$ & Regardless of use/non-use \\
\hline & $\begin{array}{l}\text { NYHA/WHO functional } \\
\text { class III/IV }\end{array}$ & TRPG $\geq 60 \mathrm{mmHg}$ & & Regardless of use/non-use \\
\hline
\end{tabular}

The highest stage satisfying all subjective symptoms, TRPG, mPAP, PVR, and use of pulmonary vasodilators or HOT is selected.

(Reference information)

If cardiac catheterization was carried out upon renewal, the value of tricuspid regurgitation pressure gradient (TRPG) determined by cardiac catheterization is used if possible.

*Precautions in performing the diagnostic criteria and severity rating

1. If the diagnostic criteria include no particular provision about the clinical symptoms, or test findings to be used for disease diagnosis, the symptoms/findings obtained at any point of time may be used (although limited to clinical symptoms presenting the course of the illness concerned and possible to be confirmed).

2. In cases in which the severity rating is done after the start of treatment, the worst condition during the latest 6 months, observed during treatment under appropriate medical management, should be judged by the physician.

3. The public healthcare expense subsidy program also covers patients whose severity level does not fall under the preset level of the above-mentioned severity classification if continuation of high-cost clinical care is needed.

\section{Document 3 Pulmonary Veno-0cclusive Disease/Pulmonary Capillary Hemangiomatosis (Listed Intractable Disease No. 87)}

\section{$<$ Criteria for Clinical Diagnosis $>$}

Major Requirements

(1) Satisfaction of the diagnostic criteria for pulmonary arterial hypertension (PAH) by right heart catheterization findings

Right heart catheterization findings upon application for new registration

(a) Increased pulmonary artery pressure (mean pulmonary artery pressure at rest $\geq 25 \mathrm{mmHg}$, pulmonary vascular resistance $\geq 3$ Wood Unit, 240 dyne $\cdot \mathrm{sec} \cdot \mathrm{cm}^{-5}$ )

(b) Pulmonary artery wedge pressure (right atrial pressure) normal $(\leq 15 \mathrm{mmHg})$

(2) Presenting high-resolution chest CT (HRCT) findings suggesting PVOD/PCH (interlobular septum hypertrophy, granular shadow, restiform shadow, ground glass opacity, mediastinal lymph node swelling) and able to rule out chronic lung disease (e.g., interstitial lung disease) and collagen disease

(3) Lung congestion/edema induced by selective pulmonary vasodilators (ERA, PDE5 inhibitor, intravenous PGI2).

\section{Secondary Requirements}

(1) Reduction of arterial oxygen tension at rest $(\leq 70 \mathrm{mmHg})$

(2) Lung function test: Marked reduction of pulmonary diffusing capability ( $\%$ DLco $<55 \%)$

(3) Pulmonary perfusion scintigraphy: Segmental perfusion defect revealed or no abnormality.

\section{Reference Findings}

(1) Hemosiderin-laden macrophages found in bronchoalveolar lavage fluid

(2) Seen more frequently in males

(3) Seen more frequently in smokers

\section{<Differential Diagnosis>}

The following diseases need to be ruled out.

Idiopathic PAH, heritable PAH, drug/toxin-induced PAH, PAH associated with various diseases (e.g., collagen disease, portal hypertension, congenital heart disease), $\mathrm{PAH}$ associated with respiratory diseases, chronic thromboembolic pulmonary hypertension. 
<Categories for Official Registration of Listed Intractable Diseases>

Registration as listed intractable disease is permitted in "definitely diagnosed cases" and "clinically diagnosed cases" described below.

In "cases suspected of having PVOD/PCH," the application should be made as cases of PAH, as a rule.

"Definitely diagnosed cases"

- Major requirement (1)(2)+ pathologically diagnosed cases

"Clinically diagnosed cases"

Satisfying any of the following requirements

- Satisfying major requirement (1)(2)+major requirement (3)+two or more secondary requirements

- Satisfying major requirement (1)(2)+all secondary requirements

"Cases suspected of having PVOD/PCH"

- Satisfying major requirement (1)(2)+one secondary requirement

$<$ Pathological Findings>

PVOD: Diffuse and marked (affecting $30-90 \%$ of veins) occlusion visible in the peripheral lung veins (particularly interlobular veins)

PCH: Multi-layering and hyperplasia of alveolar wall capillary-like small vessels, occasionally accompanied by PVOD-like peripheral lung vein lesions.

$<$ Severity Rating $>$

Stage 3 or more advanced cases are covered.

\section{(New Registration)}

\begin{tabular}{|c|c|c|c|c|}
\hline $\begin{array}{l}\text { Upon application for } \\
\text { new registration }\end{array}$ & Subjective symptoms & $\begin{array}{l}\text { Mean pulmonary artery } \\
\text { pressure (mPAP) }\end{array}$ & $\begin{array}{l}\text { Cardiac index } \\
\text { (CI) }\end{array}$ & Use of pulmonary vasodilators \\
\hline Stage 1 & $\begin{array}{l}\text { NYHA/WHO functional } \\
\text { class I/II }\end{array}$ & $40>\mathrm{mPAP} \geq 25 \mathrm{mmHg}$ & & Not used \\
\hline Stage 2 & $\begin{array}{l}\text { NYHA/WHO functional } \\
\text { class I/II }\end{array}$ & $\mathrm{mPAP} \geq 40 \mathrm{mmHg}$ & & 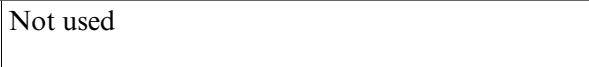 \\
\hline \multirow[t]{2}{*}{ Stage $\overline{3}$} & $\begin{array}{l}\text { NY} \overline{\mathrm{H}} \overline{\mathrm{A}} / \overline{\mathrm{W}} \overline{\mathrm{H} O} \text { functional } \\
\text { class I/II }\end{array}$ & $\overline{\mathrm{mPA}} \overline{\mathrm{P}} \geq \overline{2} \overline{\mathrm{mm}} \overline{\mathrm{Hg}}--$ & -- & Used (including past use) \\
\hline & $\begin{array}{l}\text { NYHA/WHO functional } \\
\text { class III/IV }\end{array}$ & $\mathrm{mPAP} \geq 25 \mathrm{mmHg}$ & $\begin{array}{l}\text { CI } \\
\geq 2.5 \mathrm{~L} / \mathrm{min} / \mathrm{m}^{2} \\
\end{array}$ & Regardless of use/non-use \\
\hline Stage 4 & $\begin{array}{l}\text { NYHA/WHO functional } \\
\text { class III/IV }\end{array}$ & $\mathrm{mPAP} \geq 25 \mathrm{mmHg}$ & $\begin{array}{l}\text { CI } \\
<2.5 \mathrm{~L} / \mathrm{min} / \mathrm{m}^{2}\end{array}$ & Regardless of use/non-use \\
\hline \multirow[t]{2}{*}{ Stage 5} & $\begin{array}{l}\text { NYHA/WHO functional } \\
\text { class IV }\end{array}$ & $\mathrm{mPAP} \geq 40 \mathrm{mmHg}$ & & Regardless of use/non-use \\
\hline & & & & $\begin{array}{l}\text { Stage 5, regardless of subjective symptom severity } \\
\text { and mPAP, if PGI } 2 \text { serial intravenous infusion/ } \\
\text { continued subcutaneous injection is needed }\end{array}$ \\
\hline
\end{tabular}

The highest stage satisfying all subjective symptoms, mPAP, CI, and use of pulmonary vasodilators is selected.

In cases in which the condition aggravated after use of selective pulmonary vasodilators, forcing the medication to be discontinued, Stage 3 or more advanced cases are covered even when no pulmonary vasodilator is being used (history of pulmonary vasodilator use needs to be described at the time of registration). 
(Renewal of Registration)

\begin{tabular}{|c|c|c|c|}
\hline $\begin{array}{l}\text { Upon } \\
\text { renewal }\end{array}$ & Subjective symptoms & $\begin{array}{l}\text { Tricuspid regurgitation pressure gradient } \\
\text { (TRPG) measured by echocardiography }\end{array}$ & Use of pulmonary vasodilators \\
\hline Stage 1 & $\begin{array}{l}\text { NYHA/WHO functional } \\
\text { class I/II }\end{array}$ & $\begin{array}{l}\text { TRPG }<40 \mathrm{mmHg} \\
\text { or no significant TR }\end{array}$ & Not used \\
\hline \multirow[t]{2}{*}{ Stage 2} & $\begin{array}{l}\text { NYHA/WHO functional } \\
\text { class I/II }\end{array}$ & $\mathrm{TRPG} \geq 40 \mathrm{mmHg}$ & Not used \\
\hline & $\begin{array}{l}\text { NYHA/WHO functional } \\
\text { class I }\end{array}$ & $\begin{array}{l}\text { TRPG }<40 \mathrm{mmHg} \\
\text { or no significant TR }\end{array}$ & $\begin{array}{l}\text { Used } \\
\text { (- }-1--1-1-1-1-1-1\end{array}$ \\
\hline \multirow[t]{3}{*}{ Stage $\overline{3}$} & $\begin{array}{l}\text { NY} \overline{\mathrm{H}} \overline{\mathrm{A}} / \overline{\mathrm{W}} \overline{\mathrm{H} O} \overline{\mathrm{O}} \text { functional } \\
\text { class I/II }\end{array}$ & $\overline{\mathrm{TR}} \overline{\mathrm{PG}} \geq \overline{40} \overline{\mathrm{mm}} \overline{\mathrm{Hg}}^{-}$ & Used (including past use) \\
\hline & $\begin{array}{l}\text { NYHA/WHO functional } \\
\text { class III }\end{array}$ & TRPG $\geq 40 \mathrm{mmHg}$ & Not used \\
\hline & $\begin{array}{l}\text { NYHA/WHO functional } \\
\text { class II/III }\end{array}$ & TRPG $<40 \mathrm{mmHg}$ & Used \\
\hline \multirow[t]{2}{*}{ Stage 4} & $\begin{array}{l}\text { NYHA/WHO functional } \\
\text { class II/III }\end{array}$ & $\mathrm{TRPG} \geq 60 \mathrm{mmHg}$ & Regardless of use/non-use \\
\hline & $\begin{array}{l}\text { NYHA/WHO functional } \\
\text { class IV }\end{array}$ & TRPG $<60 \mathrm{mmHg}$ & Regardless of use/non-use \\
\hline Stage 5 & $\begin{array}{l}\text { NYHA/WHO functional } \\
\text { class IV }\end{array}$ & $\mathrm{TRPG} \geq 60 \mathrm{mmHg}$ & $\begin{array}{l}\text { Regardless of use/non-use } \\
\text { If PGI } 2 \text { serial intravenous infusion/continued subcutaneous } \\
\text { injection is needed, the stage is rated at 5, regardless of } \\
\text { WHO-PH classification and mPAP }\end{array}$ \\
\hline
\end{tabular}

The highest stage satisfying all subjective symptoms, TRPG, and use of pulmonary vasodilators is selected.

In cases in which the condition aggravated after use of selective pulmonary vasodilators, forcing the medication to be discontinued, Stage 3 or more advanced cases are covered even when no pulmonary vasodilator is being used (history of pulmonary vasodilator use needs to be described at the time of registration).

(Reference information)

- In Stage 3 or more advanced cases, it is desirable to perform evaluation by cardiac catheterization at least once in 2 years. However, in patients at elevated risk involved in cardiac catheterization (e.g., children, elderly patients, and patients with multiple accompanying diseases), it is acceptable to perform echocardiographic evaluation depending on the conditions of individual cases.

- TRPG $40 \mathrm{mmHg}$ is equivalent to $\mathrm{mPAP} 25 \mathrm{mmHg}$. TRPG $60 \mathrm{mmHg}$ is equivalent to $\mathrm{mPAP} 40 \mathrm{mmHg}$.

*Precautions in performing the diagnostic criteria and severity rating

1. If the diagnostic criteria include no particular provision about the clinical symptoms, or test findings to be used for disease diagnosis, the symptoms/findings obtained at any point of time may be used (although limited to clinical symptoms presenting the course of the illness concerned and possible to be confirmed).

2. In cases in which the severity rating is done after the start of treatment, the worst condition during the latest 6 months observed during treatment under appropriate medical management should be judged by the physician.

3. The public healthcare expense subsidy program also covers patients whose severity level does not fall under the preset level of the above-mentioned severity classification if continuation of high-cost clinical care is needed.

\section{References}

1. Galiè N, Humbert M, Vachiery JL, et al. 2015 ESC/ERS Guidelines for the diagnosis and treatment of pulmonary hypertension: The Joint Task Force for the Diagnosis and Treatment of Pulmonary Hypertension of the European Society of Cardiology (ESC) and the European Respiratory Society (ERS): Endorsed by: Association for European Paediatric and Congenital Cardiology (AEPC), International Society for Heart and Lung Transplantation (ISHLT). Eur Heart J 2016; 37: 67-119. PMID: 26320113

2. Galiè N, Humbert M, Vachiery JL, et al. 2015 ESC/ERS Guidelines for the diagnosis and treatment of pulmonary hypertension: The Joint Task Force for the Diagnosis and Treatment of Pulmonary Hypertension of the European Society of Cardiology (ESC) and the European Respiratory Society (ERS): Endorsed by: Association for European Paediatric and Congenital Cardiology (AEPC), International Society for Heart and Lung Transplantation (ISHLT). Eur Respir J 2015; 46: 903-975. PMID: 26318161

3. Badesch DB, Champion HC, Sanchez MA, et al. Diagnosis and assessment of pulmonary arterial hypertension. $\mathrm{J}$ Am Coll
Cardiol 2009; 54: S55-S66. PMID: 19555859

4. Kovacs G, Berghold A, Scheidl S, et al. Pulmonary arterial pressure during rest and exercise in healthy subjects: A systematic review. Eur Respir J 2009; 34: 888-894. PMID: 19324955

5. Simonneau G, Galiè N, Rubin LJ, et al. Clinical classification of pulmonary hypertension. J Am Coll Cardiol 2004; 43: 5S-12S. PMID: 15194173

6. Rubin LJ. American College of Chest Physicians. Diagnosis and management of pulmonary arterial hypertension: ACCP evidence-based clinical practice guidelines. Chest 2004; 126: 7S-10S. PMID: 15249491

7. Simonneau G, Robbins IM, Beghetti M, et al. Updated clinical classification of pulmonary hypertension. $\mathrm{J}$ Am Coll Cardiol 2009; 54: S43-S54. PMID: 19555858

8. Simonneau G, Gatzoulis MA, Adatia I, et al. Updated clinical classification of pulmonary hypertension. $\mathrm{J}$ Am Coll Cardiol 2013; 62: D34-D41. PMID: 24355639

9. Lane KB, Machado RD, Pauciulo MW, et al. International PPH Consortium. Heterozygous germline mutations in BMPR2, encoding a TGF-beta receptor, cause familial primary pulmonary hypertension. Nat Genet 2000; 26: 81-84. PMID: 10973254

10. Trembath RC, Thomson JR, Machado RD, et al. Clinical and 
molecular genetic features of pulmonary hypertension in patients with hereditary hemorrhagic telangiectasia. $N$ Engl $J$ Med 2001; 345: 325-334. PMID: 11484689

11. Humbert M, Sitbon O, Chaouat A, et al. Pulmonary arterial hypertension in France: Results from a national registry. Am J Respir Crit Care Med 2006; 173: 1023-1030. PMID: 16456139

12. Hachulla E, Gressin V, Guillevin L, et al. Early detection of pulmonary arterial hypertension in systemic sclerosis: A French nationwide prospective multicenter study. Arthritis Rheum 2005; 52: 3792-3800. PMID: 16320330

13. Mukerjee D, St George D, Coleiro B, et al. Prevalence and outcome in systemic sclerosis associated pulmonary arterial hypertension: Application of a registry approach. Ann Rheum Dis 2003; 62: 1088-1093. PMID: 14583573

14. Yoshida S, Fukaya S. Prevalence and physiopathology of pulmonary hypertension associated with collagen vascular disease: The 2003 summary and project report of the study group on the treatment and diagnosis of intractable systemic autoimmune diseases in the Health and Labor Sciences Research Grant project for the prevention and treatment of immune-allergic diseases 2004: 40-43. [in Japanese]

15. Sitbon O, Lascoux-Combe C, Delfraissy JF, et al. Prevalence of HIV-related pulmonary arterial hypertension in the current antiretroviral therapy era. Am J Respir Crit Care Med 2008; 177: 108-113. PMID: 17932378

16. Krowka MJ, Swanson KL, Frantz RP, et al. Portopulmonary hypertension: Results from a 10-year screening algorithm. Hepatology 2006; 44: 1502-1510. PMID: 17133488

17. Hervé $\mathrm{P}$, Lebrec $\mathrm{D}$, Brenot $\mathrm{F}$, et al. Pulmonary vascular disorders in portal hypertension. Eur Respir J 1998; 11: 1153-1166. PMID: 9648972

18. Galie N, Manes A, Palazzini M, et al. Management of pulmonary arterial hypertension associated with congenital systemicto-pulmonary shunts and Eisenmenger's syndrome. Drugs 2008; 68: 1049 -1066. PMID: 18484798

19. Chaves E. The pathology of the arterial pulmonary vasculature in manson's schistosomiasis. Dis Chest 1966; 50: 72-77. PMID: 5916488

20. Montani D, Price LC, Dorfmuller P, et al. Pulmonary venoocclusive disease. Eur Respir J 2009; 33: 189-200. PMID: 19118230

21. Chaouat A, Bugnet AS, Kadaoui N, et al. Severe pulmonary hypertension and chronic obstructive pulmonary disease. $A m J$ Respir Crit Care Med 2005; 172: 189-194. PMID: 15831842

22. Colman $\mathrm{R}$, Whittingham $\mathrm{H}$, Tomlinson $\mathrm{G}$, et al. Utility of the physical examination in detecting pulmonary hypertension: A mixed methods study. PLoS One 2014; 9: e108499. PMID: 25343585

23. Leuchte HH, Holzapfel M, Baumgartner RA, et al. Clinical significance of brain natriuretic peptide in primary pulmonary hypertension. J Am Coll Cardiol 2004; 43: 764-770. PMID: 14998614

24. Fijalkowska A, Kurzyna M, Torbicki A, et al. Serum N-terminal brain natriuretic peptide as a prognostic parameter in patients with pulmonary hypertension. Chest 2006; 129: 1313-1321. PMID: 16685024

25. Voelkel MA, Wynne KM, Badesch DB, et al. Hyperuricemia in severe pulmonary hypertension. Chest 2000; 117: 19-24. PMID: 10631193

26. Li JH, Safford RE, Aduen JF, et al. Pulmonary hypertension and thyroid disease. Chest 2007; 132: 793-797. PMID: 17646226

27. Rich JD, Thenappan T, Freed B, et al. QTc prolongation is associated with impaired right ventricular function and predicts mortality in pulmonary hypertension. Int J Cardiol 2013; 167: 669-676. PMID: 22459397

28. Sun PY, Jiang X, Gomberg-Maitland M, et al. Prolonged QRS duration: A new predictor of adverse outcome in idiopathic pulmonary arterial hypertension. Chest 2012; 141: 374-380. PMID: 21778258

29. Rudski LG, Lai WW, Afilalo J, et al. Guidelines for the echocardiographic assessment of the right heart in adults: A report from the American Society of Echocardiography endorsed by the European Association of Echocardiography, a registered branch of the European Society of Cardiology, and the Canadian Society of Echocardiography. J Am Soc Echocardiogr 2010; 23: 685-713; quiz 786-788. PMID: 20620859

30. Forfia PR, Vachiéry JL. Echocardiography in pulmonary arterial hypertension. Am J Cardiol 2012; 110: 16S-24S. PMID: 22921027
31. Cottin V, Nunes H, Brillet PY, et al. Groupe d'Etude et de Recherche sur les Maladies Orphelines Pulmonaires (GERM O P). Combined pulmonary fibrosis and emphysema: A distinct underrecognised entity. Eur Respir J 2005; 26: 586-593. PMID: 16204587

32. Hughes JM, Pride NB. Examination of the carbon monoxide diffusing capacity $(\mathrm{DL}(\mathrm{CO}))$ in relation to its $\mathrm{KCO}$ and VA components. Am J Respir Crit Care Med 2012; 186: 132-139. PMID: 22538804

33. Trip P, Nossent EJ, de Man FS, et al. Severely reduced diffusion capacity in idiopathic pulmonary arterial hypertension: Patient characteristics and treatment responses. Eur Respir J 2013; 42: 1575-1585. PMID: 23949959

34. Nagaya N, Uematsu M, Satoh T, et al. Serum uric acid levels correlate with the severity and the mortality of primary pulmonary hypertension. Am J Respir Crit Care Med 1999; 160: 487-492. PMID: 10430718

35. McNicholas WT. Chronic obstructive pulmonary disease and obstructive sleep apnoea: The overlap syndrome. J Thorac Dis 2016; 8: 236-242. PMID: 26904264

36. Sugiura T, Tanabe N, Matsuura Y, et al. Role of 320-slice CT imaging in the diagnostic workup of patients with chronic thromboembolic pulmonary hypertension. Chest 2013; 143: 1070-1077. PMID: 23100061

37. Dong C, Zhou M, Liu D, et al. Diagnostic accuracy of computed tomography for chronic thromboembolic pulmonary hypertension: A systematic review and meta-analysis. PLoS One 2015; 10: $\mathrm{e}$ 0126985. PMID: 25923810

38. Bergin CJ, Sirlin C, Deutsch R, et al. Predictors of patient response to pulmonary thromboendarterectomy. AJR Am J Roentgenol 2000; 174: 509-515. PMID: 10658732

39. Resten A, Maitre S, Humbert M, et al. Pulmonary hypertension: $\mathrm{CT}$ of the chest in pulmonary venoocclusive disease. AJR Am J Roentgenol 2004; 183: 65-70. PMID: 15208112

40. Ley S, Kreitner KF, Fink C, et al. Assessment of pulmonary hypertension by CT and MR imaging. Eur Radiol 2004; 14: 359-368. PMID: 14740163

41. Edwards PD, Bull RK, Coulden R. CT measurement of main pulmonary artery diameter. $\mathrm{Br} J$ Radiol 1998; 71: 1018-1020. PMID: 10211060

42. Shin S, King CS, Brown AW, et al. Pulmonary artery size as a predictor of pulmonary hypertension and outcomes in patients with chronic obstructive pulmonary disease. Respir Med 2014; 108: 1626-1632. PMID: 25225149

43. Matsuoka S, Washko GR, Yamashiro T, et al. National Emphysema Treatment Trial Research Group. Pulmonary hypertension and computed tomography measurement of small pulmonary vessels in severe emphysema. Am J Respir Crit Care Med 2010; 181: 218-225. PMID: 19875683

44. Swift AJ, Rajaram S, Campbell MJ, et al. Prognostic value of cardiovascular magnetic resonance imaging measurements corrected for age and sex in idiopathic pulmonary arterial hypertension. Circ Cardiovasc Imaging 2014; 7: 100-106. PMID: 24275955

45. Baggen VJ, Leiner T, Post MC, et al. Cardiac magnetic resonance findings predicting mortality in patients with pulmonary arterial hypertension: A systematic review and meta-analysis. Eur Radiol 2016; 26: 3771 -3780. PMID: 26847041

46. Yamasaki Y, Nagao M, Abe K, et al. Balloon pulmonary angioplasty improves interventricular dyssynchrony in patients with inoperable chronic thromboembolic pulmonary hypertension: A cardiac MR imaging study. Int $J$ Cardiovasc Imaging 2017; 33: 229-239. PMID: 27672065

47. Sato H, Ota H, Sugimura K, et al. Balloon pulmonary angioplasty improves biventricular functions and pulmonary flow in chronic thromboembolic pulmonary hypertension. Circ J 2016; 80: 1470 - 1477. PMID: 27097557

48. Rajaram S, Swift AJ, Telfer A, et al. 3D contrast-enhanced lung perfusion MRI is an effective screening tool for chronic thromboembolic pulmonary hypertension: Results from the ASPIRE Registry. Thorax 2013; 68: 677-678. PMID: 23349220

49. Sanz J, García-Alvarez A, Fernández-Friera L, et al. Right ventriculo-arterial coupling in pulmonary hypertension: A magnetic resonance study. Heart 2012; 98: 238-243. PMID: 21917658

50. Freed BH, Gomberg-Maitland M, Chandra S, et al. Late gadolinium enhancement cardiovascular magnetic resonance predicts clinical worsening in patients with pulmonary hypertension. J Cardiovasc Magn Reson 2012; 14: 11. PMID: 22296860

51. Freed BH, Collins JD, François CJ, et al. MR and CT imaging 
for the evaluation of pulmonary hypertension. JACC Cardiovasc Imaging 2016; 9: 715-732. PMID: 27282439

52. Hoeper MM, Lee SH, Voswinckel R, et al. Complications of right heart catheterization procedures in patients with pulmonary hypertension in experienced centers. J Am Coll Cardiol 2006; 48: 2546-2552. PMID: 17174196

53. Ranu H, Smith K, Nimako K, et al. A retrospective review to evaluate the safety of right heart catheterization via the internal jugular vein in the assessment of pulmonary hypertension. Clin Cardiol 2010; 33: 303-306. PMID: 20513069

54. Taylor CJ, Derrick G, McEwan A, et al. Risk of cardiac catheterization under anaesthesia in children with pulmonary hypertension. Br J Anaesth 2007; 98: 657-661. PMID: 17401143

55. Halpern SD, Taichman DB. Misclassification of pulmonary hypertension due to reliance on pulmonary capillary wedge pressure rather than left ventricular end-diastolic pressure. Chest 2009; 136: 37-43. PMID: 19255293

56. Hoeper MM, Bogaard HJ, Condliffe R, et al. Definitions and diagnosis of pulmonary hypertension. J Am Coll Cardiol 2013; 62: D42-D50. PMID: 24355641

57. Fujimoto N, Borlaug BA, Lewis GD, et al. Hemodynamic responses to rapid saline loading: The impact of age, sex, and heart failure. Circulation 2013; 127: 55-62. PMID: 23172838

58. Hoeper MM, Maier R, Tongers J, et al. Determination of cardiac output by the Fick method, thermodilution, and acetylene rebreathing in pulmonary hypertension. Am J Respir Crit Care Med 1999; 160: 535-541. PMID: 10430725

59. Gerges C, Gerges M, Lang MB, et al. Diastolic pulmonary vascular pressure gradient: A predictor of prognosis in "out-ofproportion" pulmonary hypertension. Chest 2013; 143: 758 766. PMID: 23580984

60. Tedford RJ, Beaty CA, Mathai SC, et al. Prognostic value of the pre-transplant diastolic pulmonary artery pressure-topulmonary capillary wedge pressure gradient in cardiac transplant recipients with pulmonary hypertension. J Heart Lung Transplant 2014; 33: 289-297. PMID: 24462554

61. Sitbon O, Humbert M, Jais X, et al. Long-term response to calcium channel blockers in idiopathic pulmonary arterial hypertension. Circulation 2005; 111: 3105-3111. PMID: 15939821

62. Stein PD, Athanasoulis C, Alavi A, et al. Complications and validity of pulmonary angiography in acute pulmonary embolism. Circulation 1992; 85: 462-468. PMID: 1735144

63. Yamaki S, Kumate M, Yonesaka S, et al. Lung biopsy diagnosis of operative indication in secundum atrial septal defect with severe pulmonary vascular disease. Chest 2004; 126: 1042-1047. PMID: 15486361

64. Yamaki S. Pulmonary vascular disease associated with pulmonary hypertension in 445 patients: Diagnosis from lung biopsy and autopsy. Gen Thorac Cardiovasc Surg 2013; 61: 24-31. PMID: 23011519

65. Yamaki S, Ando M, Fukumoto Y, et al. Histopathological examination by lung biopsy for the evaluation of operability and postoperative prognosis in patients with chronic thromboembolic pulmonary hypertension. Circ $J$ 2014; 78: 476-482. PMID: 24284920

66. Jujo T, Sakao S, Ishibashi-Ueda H, et al. Evaluation of the microcirculation in chronic thromboembolic pulmonary hypertension patients: The impact of pulmonary arterial remodeling on postoperative and follow-up pulmonary arterial pressure and vascular resistance. PLoS One 2015; 10: e0133167. PMID: 26252755

67. Yamaki S, Tezuka F. Quantitative analysis of pulmonary vascular disease in complete transposition of the great arteries. Circulation 1976; 54: 805-809. PMID: 975478

68. Heath D, Edwards JE. The pathology of hypertensive pulmonary vascular disease; a description of six grades of structural changes in the pulmonary arteries with special reference to congenital cardiac septal defects. Circulation 1958; 18: 533-547. PMID: 13573570

69. Yamaki S, Horiuchi T, Miura M, et al. Secundum atrial septal defect with severe pulmonary hypertension: Open lung biopsy diagnosis of operative indication. Chest 1987; 91: 33-38. PMID: 3792083

70. Yamaki S, Ikemoto H. Operative indication based on the lung biopsy diagnosis. Clinic All-Round 2009; 58: 2216-2223. [in Japanese]

71. Miyamoto S, Nagaya N, Satoh T, et al. Clinical correlates and prognostic significance of six-minute walk test in patients with primary pulmonary hypertension: Comparison with cardiopul- monary exercise testing. Am J Respir Crit Care Med 2000; 161: 487-492. PMID: 10673190

72. Savarese G, Paolillo S, Costanzo P, et al. Do changes of 6-minute walk distance predict clinical events in patients with pulmonary arterial hypertension?: A meta-analysis of 22 randomized trials. J Am Coll Cardiol 2012; 60: 1192-1201. PMID: 22995024

73. Benza RL, Miller DP, Gomberg-Maitland M, et al. Predicting survival in pulmonary arterial hypertension: Insights from the Registry to Evaluate Early and Long-Term Pulmonary Arterial Hypertension Disease Management (REVEAL). Circulation 2010; 122: 164-172. PMID: 20585012

74. Sitbon O, Humbert M, Nunes H, et al. Long-term intravenous epoprostenol infusion in primary pulmonary hypertension: Prognostic factors and survival. J Am Coll Cardiol 2002; 40: 780-788. PMID: 12204511

75. Gabler NB, French B, Strom BL, et al. Validation of 6-minute walk distance as a surrogate end point in pulmonary arterial hypertension trials. Circulation 2012; 126: 349-356. PMID: 22696079

76. Fritz JS, Blair C, Oudiz RJ, et al. Baseline and follow-up 6-min walk distance and brain natriuretic peptide predict 2-year mortality in pulmonary arterial hypertension. Chest 2013; 143: 315-323. PMID: 22814814

77. Wensel R, Opitz CF, Anker SD, et al. Assessment of survival in patients with primary pulmonary hypertension: Importance of cardiopulmonary exercise testing. Circulation 2002; 106: 319324. PMID: 12119247

78. Oudiz RJ, Midde R, Hovenesyan A, et al. Usefulness of rightto-left shunting and poor exercise gas exchange for predicting prognosis in patients with pulmonary arterial hypertension. Am J Cardiol 2010; 105: 1186-1191. PMID: 20381675

79. McLaughlin VV, Gaine SP, Howard LS, et al. Treatment goals of pulmonary hypertension. J Am Coll Cardiol 2013; 62: D73-D81. PMID: 24355644

80. Barst RJ, McGoon M, Torbicki A, et al. Diagnosis and differential assessment of pulmonary arterial hypertension. J Am Coll Cardiol 2004; 43(12 Suppl S): 40S-47S. PMID: 15194177

81. Rich S, Dantzker DR, Ayres SM, et al. Primary pulmonary hypertension: A national prospective study. Ann Intern Med 1987; 107: 216-223. PMID: 3605900

82. D'Alonzo GE, Barst RJ, Ayres SM, et al. Survival in patients with primary pulmonary hypertension: Results from a national prospective registry. Ann Intern Med 1991; 115: 343-349. PMID: 1863023

83. Austin ED, Ma L, LeDuc C, et al. Whole exome sequencing to identify a novel gene (caveolin-1) associated with human pulmonary arterial hypertension. Circ Cardiovasc Genet 2012; 5: 336-343. PMID: 22474227

84. Thomson JR, Machado RD, Pauciulo MW, et al. Sporadic primary pulmonary hypertension is associated with germline mutations of the gene encoding BMPR-II, a receptor member of the TGF-beta family. J Med Genet 2000; 37: 741-745. PMID: 11015450

85. Nasim MT, Ogo T, Ahmed M, et al. Molecular genetic characterization of SMAD signaling molecules in pulmonary arterial hypertension. Hum Mutat 2011; 32: 1385-1389. PMID: 21898662

86. The International Primary Pulmonary Hypertension Study (IPPHS). Chest 1994; 105: 37S-41S. PMID: 8306807

87. Badesch DB, Raskob GE, Elliott CG, et al. Pulmonary arterial hypertension: Baseline characteristics from the REVEAL Registry. Chest 2010; 137: 376-387. PMID: 19837821

88. Vonk Noordegraaf A, Groeneveldt JA, Bogaard HJ. Pulmonary hypertension. Eur Respir Rev 2016; 25: 4-11. PMID: 26929415

89. Humbert M, Ghofrani HA. The molecular targets of approved treatments for pulmonary arterial hypertension. Thorax 2016; 71: 73-83. PMID: 26219978

90. Humbert M, Lau EM, Montani D, et al. Advances in therapeutic interventions for patients with pulmonary arterial hypertension. Circulation 2014; 130: 2189-2208. PMID: 25602947

91. Evans JD, Girerd B, Montani D, et al. BMPR2 mutations and survival in pulmonary arterial hypertension: An individual participant data meta-analysis. Lancet Respir Med 2016; 4: 129-137. PMID: 26795434

92. Atkinson C, Stewart S, Upton PD, et al. Primary pulmonary hypertension is associated with reduced pulmonary vascular expression of type II bone morphogenetic protein receptor. Circulation 2002; 105: 1672-1678. PMID: 11940546

93. Spiekerkoetter E, Sung YK, Sudheendra D, et al. Low-dose FK506 (Tacrolimus) in end-stage pulmonary arterial hyperten- 
sion. Am J Respir Crit Care Med 2015; 192: 254-257. PMID: 26177174

94. Long L, Ormiston ML, Yang X, et al. Selective enhancement of endothelial BMPR-II with BMP9 reverses pulmonary arterial hypertension. Nat Med 2015; 21: 777-785. PMID: 26076038

95. Okada O, Tanabe N, Yasuda J, et al. Prediction of life expectancy in patients with primary pulmonary hypertension. A retrospective nationwide survey from 1980-1990. Intern Med 1999; 38: 12-16. PMID: 10052735

96. Humbert M, Sitbon O, Yaïci A, et al. French Pulmonary Arterial Hypertension Network. Survival in incident and prevalent cohorts of patients with pulmonary arterial hypertension. Eur Respir J 2010; 36: 549-555. PMID: 20562126

97. Benza RL, Miller DP, Barst RJ, et al. An evaluation of longterm survival from time of diagnosis in pulmonary arterial hypertension from the REVEAL Registry. Chest 2012; 142: 448-456. PMID: 22281797

98. Ogawa A, Satoh T, Tamura Y, et al. Survival of Japanese patients with idiopathic/heritable pulmonary arterial hypertension. Am J Cardiol 2017; 119: 1479-1484. PMID: 28267959

99. Oudiz RJ, Barst RJ, Hansen JE, et al. Cardiopulmonary exercise testing and six-minute walk correlations in pulmonary arterial hypertension. Am J Cardiol 2006; 97: 123-126. PMID: 16377296

100. Forfia PR, Fisher MR, Mathai SC, et al. Tricuspid annular displacement predicts survival in pulmonary hypertension. $\mathrm{Am}$ J Respir Crit Care Med 2006; 174: 1034-1041. PMID: 16888289

101. Nagaya N, Nishikimi T, Uematsu M, et al. Plasma brain natriuretic peptide as a prognostic indicator in patients with primary pulmonary hypertension. Circulation 2000; 102: 865-870. PMID: 10952954

102. Benza RL, Gomberg-Maitland M, Miller DP, et al. The REVEAL Registry risk score calculator in patients newly diagnosed with pulmonary arterial hypertension. Chest 2012; 141: $354-362$. PMID: 21680644

103. Ogawa A, Ejiri K, Matsubara H. Long-term patient survival with idiopathic/heritable pulmonary arterial hypertension treated at a single center in Japan. Life Sci 2014; 118: 414-419. PMID: 24530872

104. Fujiwara M, Yagi H, Matsuoka R, et al. Implications of mutations of activin receptor-like kinase 1 gene (ALK1) in addition to bone morphogenetic protein receptor II gene (BMPR2) in children with pulmonary arterial hypertension. Circ J 2008; 72: 127-133. PMID: 18159113

105. Shintani M, Yagi H, Nakayama T, et al. A new nonsense mutation of SMAD8 associated with pulmonary arterial hypertension. J Med Genet 2009; 46: 331 -337. PMID: 19211612

106. Li X, Zhang X, Leathers R, et al. Notch3 signaling promotes the development of pulmonary arterial hypertension. Nat Med 2009; 15: 1289-1297. PMID: 19855400

107. Yu Y, Keller SH, Remillard CV, et al. A functional singlenucleotide polymorphism in the TRPC6 gene promoter associated with idiopathic pulmonary arterial hypertension. Circulation 2009; 119: 2313-2322. PMID: 19380626

108. Katano H, Ito K, Shibuya K, et al. Lack of human herpesvirus 8 infection in lungs of Japanese patients with primary pulmonary hypertension. J Infect Dis 2005; 191: 743-745. PMID: 15688289

109. Soubrier F, Chung WK, Machado R, et al. Genetics and genomics of pulmonary arterial hypertension. J Am Coll Cardiol 2013; 62: D13-D21. PMID: 24355637

110. Kataoka M, Aimi Y, Yanagisawa R, et al. Alu-mediated nonallelic homologous and nonhomologous recombination in the BMPR2 gene in heritable pulmonary arterial hypertension. Genet Med 2013; 15: 941-947. PMID: 23579436

111. Momose Y, Aimi Y, Hirayama T, et al. De novo mutations in the BMPR2 gene in patients with heritable pulmonary arterial hypertension. Ann Hum Genet 2015; 79: 85-91. PMID: 25612240

112. Isobe $\mathrm{S}$, Kataoka $\mathrm{M}$, Aimi $\mathrm{Y}$, et al. Improved survival of patients with pulmonary arterial hypertension with BMPR2 mutations in the last decade. Am J Respir Crit Care Med 2016; 193: 1310 -1314. PMID: 27248591

113. Mereles D, Ehlken N, Kreuscher S, et al. Exercise and respiratory training improve exercise capacity and quality of life in patients with severe chronic pulmonary hypertension. Circulation 2006; 114: 1482-1489. PMID: 16982941

114. Weinstein AA, Chin LM, Keyser RE, et al. Effect of aerobic exercise training on fatigue and physical activity in patients with pulmonary arterial hypertension. Respir Med 2013; 107: 778 784. PMID: 23478192

115. Chan L, Chin LMK, Kennedy M, et al. Benefits of intensive treadmill exercise training on cardiorespiratory function and quality of life in patients with pulmonary hypertension. Chest 2013; 143: 333-343. PMID: 22922554

116. Ehlken N, Lichtblau M, Klose $\mathrm{H}$, et al. Exercise training improves peak oxygen consumption and haemodynamics in patients with severe pulmonary arterial hypertension and inoperable chronic thrombo-embolic pulmonary hypertension: A prospective, randomized, controlled trial. Eur Heart J 2016; 37: 35-44. PMID: 26231884

117. de Man FS, Handoko ML, Groepenhoff H, et al. Effects of exercise training in patients with idiopathic pulmonary arterial hypertension. Eur Respir J 2009; 34: 669-675. PMID: 19720810

118. Grünig E, Ehlken N, Ghofrani A, et al. Effect of exercise and respiratory training on clinical progression and survival in patients with severe chronic pulmonary hypertension. Respiration 2011; 81: 394-401. PMID: 21311162

119. Grünig E, Maier F, Ehlken N, et al. Exercise training in pulmonary arterial hypertension associated with connective tissue diseases. Arthritis Res Ther 2012; 14: R148. PMID: 22709477

120. Grünig E, Lichtblau M, Ehlken N, et al. Safety and efficacy of exercise training in various forms of pulmonary hypertension. Eur Respir J 2012; 40: 84-92. PMID: 22323570

121. Handoko ML, de Man FS, Happé CM, et al. Opposite effects of training in rats with stable and progressive pulmonary hypertension. Circulation 2009; 120: 42-49. PMID: 19546388

122. Thorne S, Nelson-Piercy C, MacGregor A, et al. Pregnancy and contraception in heart disease and pulmonary arterial hypertension. J Fam Plann Reprod Health Care 2006; 32: 75-81. PMID: 16824295

123. Meyer S, McLaughlin VV, Seyfarth HJ, et al. Outcomes of noncardiac, nonobstetric surgery in patients with PAH: An international prospective survey. Eur Respir J 2013; 41: 1302 1307. PMID: 23143546

124. Weitzenblum E, Sautegeau A, Ehrhart M, et al. Long-term oxygen therapy can reverse the progression of pulmonary hypertension in patients with chronic obstructive pulmonary disease. Am Rev Respir Dis 1985; 131: 493-498. PMID: 3922267

125. Rich S, Kaufmann E, Levy PS. The effect of high doses of calcium-channel blockers on survival in primary pulmonary hypertension. N Engl J Med 1992; 327: 76-81. PMID: 1603139

126. Fuster V, Steele PM, Edwards WD, et al. Primary pulmonary hypertension: Natural history and the importance of thrombosis. Circulation 1984; 70: 580-587. PMID: 6148159

127. Frank H, Mlczoch J, Huber K, et al. The effect of anticoagulant therapy in primary and anorectic drug-induced pulmonary hypertension. Chest 1997; 112: 714-721. PMID: 9315805

128. Tamura Y, Kimura M, Takei M, et al. Oral vasopressin receptor antagonist tolvaptan in right heart failure due to pulmonary hypertension. Eur Respir J 2015; 46: 283-286. PMID: 25929949

129. Sato K, Okada O, Tanabe N, et al. Pulmonary homodynamic and gas exchange effects of various oxygen concentrations in patients with severe pulmonary hypertension primarily affecting the pulmonary vasculature. The Journal of the Japanese Respiratory Society 1999; 37: 449-457. [in Japanese]

130. Roberts DH, Lepore JJ, Maroo A, et al. Oxygen therapy improves cardiac index and pulmonary vascular resistance in patients with pulmonary hypertension. Chest 2001; 120: $1547-$ 1555. PMID: 11713133

131. Sandoval J, Aguirre JS, Pulido T, et al. Nocturnal oxygen therapy in patients with the Eisenmenger syndrome. Am J Respir Crit Care Med 2001; 164: 1682-1687. PMID: 11719310

132. Kim YS, Aviado DM. Digitalis and the pulmonary circulation. Am Heart J 1961; 62: 680-686. PMID: 14455984

133. Rich S, Seidlitz M, Dodin E, et al. The short-term effects of digoxin in patients with right ventricular dysfunction from pulmonary hypertension. Chest 1998; 114: 787-792. PMID: 9743167

134. Rhodes CJ, Howard LS, Busbridge M, et al. Iron deficiency and raised hepcidin in idiopathic pulmonary arterial hypertension: Clinical prevalence, outcomes, and mechanistic insights. $J$ Am Coll Cardiol 2011; 58: 300-309. PMID: 21737024

135. Farber HW, Miller DP, Poms AD, et al. Five-year outcomes of patients enrolled in the REVEAL Registry. Chest 2015; 148: 1043-1054. PMID: 26066077

136. Tamura Y, Kumamaru H, Satoh T, et al. Effectiveness and outcome of pulmonary arterial hypertension-specific therapy in Japanese patients with pulmonary arterial hypertension. Circ J 2017; 82: 275-282. PMID: 28747612

137. Sitbon O, Channick R, Chin KM, et al. GRIPHON Investigators. Selexipag for the treatment of pulmonary arterial hypertension. N Engl J Med 2015; 373: 2522-2533. PMID: 26699168 
138. Pulido T, Adzerikho I, Channick RN, et al. SERAPHIN Investigators. Macitentan and morbidity and mortality in pulmonary arterial hypertension. $N$ Engl J Med 2013; 369: 809-818. PMID: 23984728

139. Langleben $\mathrm{D}$, Galiè $\mathrm{N}, \mathrm{He} \mathrm{J}$, et al. Use of clinically relevant responder threshold criteria to evaluate the response to treatment in the phase III PATENT-1 study. J Heart Lung Transplant 2015; 34: 338-347. PMID: 25703961

140. Rubin LJ, Galiè N, Grimminger F, et al. Riociguat for the treatment of pulmonary arterial hypertension: A long-term extension study (PATENT-2). Eur Respir J 2015; 45: 1303 1313. PMID: 25614164

141. Nickel N, Golpon H, Greer M, et al. The prognostic impact of follow-up assessments in patients with idiopathic pulmonary arterial hypertension. Eur Respir J 2012; 39: 589-596. PMID: 21885392

142. McLaughlin VV, Presberg KW, Doyle RL, et al. American College of Chest Physicians. Prognosis of pulmonary arterial hypertension: ACCP evidence-based clinical practice guidelines. Chest 2004; 126: 78S-92S. PMID: 15249497

143. Hoeper MM, Markevych I, Spiekerkoetter E, et al. Goaloriented treatment and combination therapy for pulmonary arterial hypertension. Eur Respir J 2005; 26: 858-863. PMID: 16264047

144. Galiè N, Barberà JA, Frost AE, et al. AMBITION Investigators. Initial use of ambrisentan plus tadalafil in pulmonary arterial hypertension. $N$ Engl J Med 2015; 373: 834-844. PMID: 26308684

145. Sitbon O, Jaïs X, Savale L, et al. Upfront triple combination therapy in pulmonary arterial hypertension: A pilot study. Eur Respir J 2014; 43: 1691-1697. PMID: 24627535

146. Venitz J, Zack J, Gillies H, et al. Clinical pharmacokinetics and drug-drug interactions of endothelin receptor antagonists in pulmonary arterial hypertension. J Clin Pharmacol 2012; 52: 1784-1805. PMID: 22205719

147. Actelion Pharmaceuticals Japan Ltd. Endothelin receptor antagonist Tracleer ${ }^{\circledR}$ tablets $62.5 \mathrm{mg}$ interview form 2013: 1-44. [in Japanese]

148. Corona G, Razzoli E, Forti G, et al. The use of phosphodiesterase 5 inhibitors with concomitant medications. J Endocrinol Invest 2008; 31: 799-808. PMID: 18997493

149. Burgess G, Hoogkamer H, Collings L, et al. Mutual pharmacokinetic interactions between steady-state bosentan and sildenafil. Eur J Clin Pharmacol 2008; 64: 43-50. PMID: 18040672

150. Paul GA, Gibbs JS, Boobis AR, et al. Bosentan decreases the plasma concentration of sildenafil when coprescribed in pulmonary hypertension. Br J Clin Pharmacol 2005; 60: 107-112. PMID: 15963102

151. Gruenig E, Michelakis E, Vachiéry JL, et al. Acute hemodynamic effects of single-dose sildenafil when added to established bosentan therapy in patients with pulmonary arterial hypertension: Results of the COMPASS-1 study. J Clin Pharmacol 2009; 49: 1343-1352. PMID: 19755415

152. McLaughlin V, Channick RN, Ghofrani HA, et al. Bosentan added to sildenafil therapy in patients with pulmonary arterial hypertension. Eur Respir J 2015; 46: 405-413. PMID: 26113687

153. Nippon Shinyaku Co. Phosphodiesterase 5 inhibitor Adcirca ${ }^{\circledR}$ tablets $20 \mathrm{mg}$ interview form 2011. [in Japanese]

154. GlaxoSmithKline K.K. Endothelin receptor antagonist Volibris $\mathbb{}$ tablets $2.5 \mathrm{mg}$ interview form 2014: 1-60. [in Japanese]

155. Spence R, Mandagere A, Harrison B, et al. No clinically relevant pharmacokinetic and safety interactions of ambrisentan in combination with tadalafil in healthy volunteers. J Pharm Sci 2009; 98: 4962-4974. PMID: 19455620

156. Spence R, Mandagere A, Dufton C, et al. Pharmacokinetics and safety of ambrisentan in combination with sildenafil in healthy volunteers. J Clin Pharmacol 2008; 48: 1451-1459. PMID: 18832294

157. Pfizer Japan Inc. Phosphodiesterase 5 inhibitor Revatio $₫$ tablets $20 \mathrm{mg}$ interview form 2016. [in Japanese]

158. European Medicines Agency. Adempus (riociguat) tablets. Aneex I: summary of product charcteristics. Adempas: EPAR - Product Information. http://www.ema.europa.eu/docs/en_GB/document library/EPAR_-_Product_Information/human/002737/ WC500165034.pdf

159. Bayer HealthCare Pharmaceuticals. Adempus (riociguat) tablets. Prescribing information. http://labeling.bayerhealthcare.com/ html/products/pi/Adempas_PI.pdf

160. Hoeper MM, Granton J. Intensive care unit management of patients with severe pulmonary hypertension and right heart failure. Am J Respir Crit Care Med 2011; 184: 1114-1124. PMID: 21700906

161. Braun EB, Palin CA, Hogue CW. Vasopressin during spinal anesthesia in a patient with primary pulmonary hypertension treated with intravenous epoprostenol. Anesth Analg 2004; 99: 36-37. PMID: 15281498

162. Price LC, Forrest P, Sodhi V, et al. Use of vasopressin after Caesarean section in idiopathic pulmonary arterial hypertension. Br J Anaesth 2007; 99: 552-555. PMID: 17660458

163. Tayama E, Ueda T, Shojima T, et al. Arginine vasopressin is an ideal drug after cardiac surgery for the management of low systemic vascular resistant hypotension concomitant with pulmonary hypertension. Interact Cardiovasc Thorac Surg 2007; 6: 715-719. PMID: 17704123

164. Olsson KM, Simon A, Strueber M, et al. Extracorporeal membrane oxygenation in nonintubated patients as bridge to lung transplantation. Am J Transplant 2010; 10: 2173-2178. PMID: 20636463

165. Rosenzweig EB, Brodie D, Abrams DC, et al. Extracorporeal membrane oxygenation as a novel bridging strategy for acute right heart failure in group 1 pulmonary arterial hypertension. ASAIO J 2014; 60: 129-133. PMID: 24299971

166. Pavlushkov E, Berman M, Valchanov K. Cannulation techniques for extracorporeal life support. Ann Transl Med 2017; 5: 70. PMID: 28275615

167. Weill D, Benden C, Corris PA, et al. A consensus document for the selection of lung transplant candidates: 2014--an update from the Pulmonary Transplantation Council of the International Society for Heart and Lung Transplantation. J Heart Lung Transplant 2015; 34: 1-15. PMID: 25085497

168. Yusen RD, Edwards LB, Dipchand AI, et al. International Society for Heart and Lung Transplantation. The Registry of the International Society for Heart and Lung Transplantation: Thirty-third adult lung and heart-lung transplant report-2016; Focus theme: Primary diagnostic indications for transplant. $J$ Heart Lung Transplant 2016; 35: 1170-1184. PMID: 27772669

169. Daliento L, Somerville J, Presbitero P, et al. Eisenmenger syndrome. Factors relating to deterioration and death. Eur Heart J 1998; 19: 1845-1855. PMID: 9886728

170. Spray TL, Mallory GB, Canter CE, et al. Pediatric lung transplantation for pulmonary hypertension and congenital heart disease. Ann Thorac Surg 1992; 54: 216-223; discussion 224225. PMID: 1637208

171. Showkathali R, Tayebjee MH, Grapsa J, et al. Right atrial flutter isthmus ablation is feasible and results in acute clinical improvement in patients with persistent atrial flutter and severe pulmonary arterial hypertension. Int J Cardiol 2011; 149: 279-280. PMID: 21420184

172. Tongers J, Schwerdtfeger B, Klein G, et al. Incidence and clinical relevance of supraventricular tachyarrhythmias in pulmonary hypertension. Am Heart J 2007; 153: 127-132. PMID: 17174650

173. Żyłkowska J, Kurzyna M, Pietura R, et al. Recurrent hemoptysis: An emerging life-threatening complication in idiopathic pulmonary arterial hypertension. Chest 2011; 139: 690-693. PMID: 21362657

174. Żyłkowska J, Kurzyna M, Florczyk M, et al. Pulmonary artery dilatation correlates with the risk of unexpected death in chronic arterial or thromboembolic pulmonary hypertension. Chest 2012; 142: 1406-1416. PMID: 22797193

175. Russo V, Zompatori M, Galiè N. Extensive right pulmonary artery dissection in a young patient with chronic pulmonary hypertension. Heart 2012; 98: 265-266. PMID: 22155730

176. Demerouti EA, Manginas AN, Athanassopoulos GD, et al. Complications leading to sudden cardiac death in pulmonary arterial hypertension. Respir Care 2013; 58: 1246-1254. PMID: 23271814

177. Lee MS, Oyama J, Bhatia R, et al. Left main coronary artery compression from pulmonary artery enlargement due to pulmonary hypertension: A contemporary review and argument for percutaneous revascularization. Catheter Cardiovasc Interv 2010; 76: 543-550. PMID: 20506194

178. Doran AK, Ivy DD, Barst RJ, et al. Scientific Leadership Council of the Pulmonary Hypertension Association. Guidelines for the prevention of central venous catheter-related blood stream infections with prostanoid therapy for pulmonary arterial hypertension. Int J Clin Pract Suppl 2008; (160): 5-9. PMID: 18638170

179. Guidelines for Diagnosis and Treatment of Cardiovascular Diseases. Statement for end-stage cardiovascular care (JCS 2010). http://www.j-circ.or.jp/guideline/pdf/JCS2010_nonogi_h. 
pdf [in Japanese]

180. Grinnan DC, Swetz KM, Pinson J, et al. The end-of-life experience for a cohort of patients with pulmonary arterial hypertension. J Palliat Med 2012; 15: 1065-1070. PMID: 22845004

181. Tonelli AR, Arelli V, Minai OA, et al. Causes and circumstances of death in pulmonary arterial hypertension. Am J Respir Crit Care Med 2013; 188: 365-369. PMID: 23600433

182. The Ministry of Health, Labour and Welfare. Guidelines for decision making with end-of-life care (Published in May 2007 and revised in March 2015) http://www.mhlw.go.jp/file/ 06-Seisakujouhou-10800000-Iseikyoku/0000078981.pdf [in Japanese]

183. Saunders C. The management of terminal malignant disease, 2nd edn. Edward Arnold, 1984, 232-241.

184. Eric R. Fenstad ER, Wordingham SE, Swetz KM. Pulmonary hypertension and palliative care: What, when, where, and why? Adv Pulm Hypertens 2016; 15: 26-31.

185. Wordingham SE, Swetz KM. Overview of palliative care and hospice services. Clin Liver Dis 2015; 6: 30-32.

186. Delcroix M, Howard L. Pulmonary arterial hypertension: The burden of disease and impact on quality of life. Eur Respir Rev 2015; 24: 621-629. PMID: 26621976

187. Abernethy AP, Currow DC, Frith P, et al. Randomised, double blind, placebo controlled crossover trial of sustained release morphine for the management of refractory dyspnoea. $B M J$ 2003; 327: 523-528. PMID: 12958109

188. Ekström MP, Abernethy AP, Currow DC. The management of chronic breathlessness in patients with advanced and terminal illness. BMJ 2015; 349: g7617. PMID: 25556037

189. Rival G, Lacasse Y, Martin S, et al. Effect of pulmonary arterial hypertension-specific therapies on health-related quality of life: A systematic review. Chest 2014; 146: 686-708. PMID: 24626791

190. Guillevin L, Armstrong I, Aldrighetti R, et al. Understanding the impact of pulmonary arterial hypertension on patients' and carers' lives. Eur Respir Rev 2013; 22: 535-542. PMID: 24293469

191. M M Vanhoof J, Delcroix M, Vandevelde E, et al. Emotional symptoms and quality of life in patients with pulmonary arterial hypertension. J Heart Lung Transplant 2014; 33: 800-808. PMID: 24854567

192. Palliative Medicine Guideline Preparation Committee of the Japanese Society for Palliative Medicine. Guidelines for palliative sedation 2010. https://www.jspm.ne.jp/guidelines/sedation/2010/ index.php [in Japanese]

193. Maeda I, Morita T, Yamaguchi T, et al. Effect of continuous deep sedation on survival in patients with advanced cancer (J-Proval): A propensity score-weighted analysis of a prospective cohort study. Lancet Oncol 2016; 17: 115-122. PMID: 26610854

194. Schermuly RT, Dony E, Ghofrani HA, et al. Reversal of experimental pulmonary hypertension by PDGF inhibition. J Clin Invest 2005; 115: 2811 -2821. PMID: 16200212

195. Hoeper MM, Barst RJ, Bourge RC, et al. Imatinib mesylate as add-on therapy for pulmonary arterial hypertension: Results of the randomized IMPRES study. Circulation 2013; 127: 1128-1138. PMID: 23403476

196. Gomberg-Maitland M, Bull TM, Saggar R, et al. New trial designs and potential therapies for pulmonary artery hypertension. J Am Coll Cardiol 2013; 62: D82-D91. PMID: 24355645

197. Pietra GG, Capron F, Stewart S, et al. Pathologic assessment of vasculopathies in pulmonary hypertension. $\mathrm{J}$ Am Coll Cardiol 2004; 43: 25S-32S. PMID: 15194175

198. Tuder RM, Abman SH, Braun T, et al. Development and pathology of pulmonary hypertension. J Am Coll Cardiol 2009; 54: S3-S9. PMID: 19555856

199. Todorovich-Hunter L, Johnson DJ, Ranger P, et al. Altered elastin and collagen synthesis associated with progressive pulmonary hypertension induced by monocrotaline: A biochemical and ultrastructural study. Lab Invest 1988; 58: 184-195. PMID: 3123799

200. Yang X, Mardekian J, Sanders KN, et al. Prevalence of pulmonary arterial hypertension in patients with connective tissue diseases: A systematic review of the literature. Clin Rheumatol 2013; 32: 1519-1531. PMID: 23780636

201. Tojo K, Akiya K, Torikai K, et al. A nationwide epidemiological survey on the complication rate of pulmonary hypertension in patients with mixed connective tissue disease, systemic lupus erythematosus, systemic sclerosis and polymyositis dermatomyositis. The 1998 report of the mixed connective tissue disease taskforce of the skin and connective tissue disease study group of the MHLW 1999: 3-6. [in Japanese]
202. Allcock RJ, Forrest I, Corris PA, et al. A study of the prevalence of systemic sclerosis in northeast England. Rheumatology (Oxford) 2004; 43: 596-602. PMID: 14872101

203. Mayes MD. Scleroderma epidemiology. Rheum Dis Clin North Am 2003; 29: 239-254. PMID: 12841293

204. Avouac J, Airò P, Meune C, et al. Prevalence of pulmonary hypertension in systemic sclerosis in European Caucasians and metaanalysis of 5 studies. J Rheumatol 2010; 37: 2290-2298. PMID: 20810505

205. Pérez-Peñate GM, Rúa-Figueroa I, Juliá-Serdá G, et al. Pulmonary arterial hypertension in systemic lupus erythematosus: Prevalence and predictors. J Rheumatol 2016; 43: 323-329. PMID: 26669915

206. Li M, Wang Q, Zhao J, et al. CSTAR co-authors. Chinese SLE Treatment and Research group (CSTAR) registry: II. Prevalence and risk factors of pulmonary arterial hypertension in Chinese patients with systemic lupus erythematosus. Lupus 2014; 23: 1085-1091. PMID: 24651670

207. Launay D, Hachulla E, Hatron PY, et al. Pulmonary arterial hypertension: A rare complication of primary Sjögren syndrome: Report of 9 new cases and review of the literature. Medicine (Baltimore) 2007; 86: 299-315. PMID: 17873760

208. Condliffe R, Kiely DG, Peacock AJ, et al. Connective tissue disease-associated pulmonary arterial hypertension in the modern treatment era. Am J Respir Crit Care Med 2009; 179: 151-157. PMID: 18931333

209. Shirai Y, Yasuoka H, Okano Y, et al. Clinical characteristics and survival of Japanese patients with connective tissue disease and pulmonary arterial hypertension: A single-centre cohort. Rheumatology (Oxford) 2012; 51: 1846-1854. PMID: 22740623

210. Giordano N, Montella A, Corallo C, et al. Pulmonary hypertension: A correct diagnosis for a suitable therapy in scleroderma patients. Clin Exp Rheumatol 2015; 33: S182-S189. PMID: 26339897

211. Hachulla E, de Groote P, Gressin V, et al. Itinér AIRSclérodermie Study Group. The three-year incidence of pulmonary arterial hypertension associated with systemic sclerosis in a multicenter nationwide longitudinal study in France. Arthritis Rheum 2009; 60: 1831-1839. PMID: 19479881

212. Hinchcliff M, Fischer A, Schiopu E, et al. PHAROS Investigators. Pulmonary Hypertension Assessment and Recognition of Outcomes in Scleroderma (PHAROS): Baseline characteristics and description of study population. J Rheumatol 2011; 38: 2172-2179. PMID: 21844142

213. Hurdman J, Condliffe R, Elliot CA, et al. ASPIRE registry: Assessing the Spectrum of Pulmonary hypertension Identified at a REferral centre. Eur Respir J 2012; 39: 945-955. PMID: 21885399

214. Rubenfire M, Huffman MD, Krishnan S, et al. Survival in systemic sclerosis with pulmonary arterial hypertension has not improved in the modern era. Chest 2013; 144: 1282-1290. PMID: 23744060

215. Chung L, Liu J, Parsons L, et al. Characterization of connective tissue disease-associated pulmonary arterial hypertension from REVEAL: Identifying systemic sclerosis as a unique phenotype. Chest 2010; 138: 1383-1394. PMID: 20507945

216. Asano Y, Jinnin M, Kawaguchi Y, et al. Guidelines for diagnosis, severity classification, and treatment of systemic scleroderma. The Japanese Journal of Dermatology 2016; 126: 1831 - 1896. [in Japanese]

217. Humbert M, Yaici A, de Groote P, et al. Screening for pulmonary arterial hypertension in patients with systemic sclerosis: Clinical characteristics at diagnosis and long-term survival. Arthritis Rheum 2011; 63: 3522-3530. PMID: 21769843

218. Launay D, Sitbon O, Hachulla E, et al. Survival in systemic sclerosis-associated pulmonary arterial hypertension in the modern management era. Ann Rheum Dis 2013; 72: 1940-1946. PMID: 23178295

219. Campo A, Mathai SC, Le Pavec J, et al. Hemodynamic predictors of survival in scleroderma-related pulmonary arterial hypertension. Am J Respir Crit Care Med 2010; 182: 252-260. PMID: 20339143

220. Hinchcliff M, Khanna S, Hsu VM, et al. PHAROS Investigators. Survival in systemic sclerosis-pulmonary arterial hypertension by serum autoantibody status in the Pulmonary Hypertension Assessment and Recognition of Outcomes in Scleroderma (PHAROS) Registry. Semin Arthritis Rheum 2015; 45: 309-314. PMID: 26210782

221. Sobanski V, Giovannelli J, Lynch BM, et al. Characteristics and survival of anti-U1 RNP antibody-positive patients with 
connective tissue disease-associated pulmonary arterial hypertension. Arthritis Rheum 2016; 68: 484-493. PMID: 26415038

222. Torikai K, Fukaya S, Matsumoto Y, et al. Prognosis of patients with pulmonary hypertension associated with mixed connective tissue disease: The 1998 report of the mixed connective tissue disease taskforce of the skin and connective tissue disease study group of the MHLW 1999: 20-23. [in Japanese]

223. Fischer A, Bull TM, Steen VD. Practical approach to screening for scleroderma-associated pulmonary arterial hypertension. Arthritis Care Res (Hoboken) 2012; 64: 303-310. PMID: 22076819

224. Stupi AM, Steen VD, Owens GR, et al. Pulmonary hypertension in the CREST syndrome variant of systemic sclerosis. Arthritis Rheum 1986; 29: 515-524. PMID: 3707629

225. Steen V, Medsger TA. Predictors of isolated pulmonary hypertension in patients with systemic sclerosis and limited cutaneous involvement. Arthritis Rheum 2003; 48: 516-522. PMID: 12571862

226. Coghlan JG, Denton CP, Grünig E, et al. DETECT study group. Evidence-based detection of pulmonary arterial hypertension in systemic sclerosis: The DETECT study. Ann Rheum Dis 2014; 73: 1340-1349. PMID: 23687283

227. Shah AA, Wigley FM, Hummers LK. Telangiectases in scleroderma: A potential clinical marker of pulmonary arterial hypertension. J Rheumatol 2010; 37: 98-104. PMID: 19955048

228. Hurabielle C, Avouac J, Lepri G, et al. Skin telangiectasia and the identification of a subset of systemic sclerosis patients with severe vascular disease. Arthritis Care Res (Hoboken) 2016; 68: 1021 - 1027. PMID: 26474084

229. Mukerjee D, St George D, Knight C, et al. Echocardiography and pulmonary function as screening tests for pulmonary arterial hypertension in systemic sclerosis. Rheumatology (Oxford) 2004; 43: 461-466. PMID: 15024134

230. Steen VD, Graham G, Conte C, et al. Isolated diffusing capacity reduction in systemic sclerosis. Arthritis Rheum 1992; 35: 765-770. PMID: 1622414

231. Hsu VM, Chung L, Hummers LK, et al. Development of pulmonary hypertension in a high-risk population with systemic sclerosis in the Pulmonary Hypertension Assessment and Recognition of Outcomes in Scleroderma (PHAROS) cohort study. Semin Arthritis Rheum 2014; 44: 55-62. PMID: 24709277

232. Cavagna L, Caporali R, Klersy C, et al. Comparison of brain natriuretic peptide (BNP) and NT-proBNP in screening for pulmonary arterial hypertension in patients with systemic sclerosis. J Rheumatol 2010; 37: 2064-2070. PMID: 20634241

233. Dimitroulas T, Giannakoulas G, Dimitroula H, et al. Significance of serum uric acid in pulmonary hypertension due to systemic sclerosis: A pilot study. Rheumatol Int 2011; 31: 263-267. PMID: 20658290

234. Kim KJ, Baek IW, Park YJ, et al. High levels of uric acid in systemic lupus erythematosus is associated with pulmonary hypertension. Int $J$ Rheum Dis 2015; 18: 524-532. PMID: 24428867

235. Yoshida S, Fukaya S, Kyotani S, et al. Revised guidelines for the diagnosis of pulmonary arterial hypertension (PAH) associated with mixed connective tissue disease (MCTD): The 2010 report of the study group on mixed connective tissue disease in the Health and Labor Sciences Research Grant for intractable diseases. 2011, 7-13. [in Japanese]

236. Overbeek MJ, Vonk MC, Boonstra A, et al. Pulmonary arterial hypertension in limited cutaneous systemic sclerosis: A distinctive vasculopathy. Eur Respir J 2009; 34: 371-379. PMID: 19282344

237. Günther S, Jaïs X, Maitre S, et al. Computed tomography findings of pulmonary venoocclusive disease in scleroderma patients presenting with precapillary pulmonary hypertension. Arthritis Rheum 2012; 64: 2995-3005. PMID: 22549387

238. Fernandes F, Ramires FJ, Arteaga E, et al. Cardiac remodeling in patients with systemic sclerosis with no signs or symptoms of heart failure: An endomyocardial biopsy study. J Card Fail 2003; 9: 311-317. PMID: 13680552

239. Valentini G, Vitale DF, Giunta A, et al. Diastolic abnormalities in systemic sclerosis: Evidence for associated defective cardiac functional reserve. Ann Rheum Dis 1996; 55: 455-460. PMID: 8774164

240. Tzelepis GE, Kelekis NL, Plastiras SC, et al. Pattern and distribution of myocardial fibrosis in systemic sclerosis: A delayed enhanced magnetic resonance imaging study. Arthritis Rheum 2007; 56: 3827-3836. PMID: 17968945

241. Bulkley BH, Ridolfi RL, Salyer WR, et al. Myocardial lesions of progressive systemic sclerosis: A cause of cardiac dysfunction.
Circulation 1976; 53: 483-490. PMID: 1248080

242. Steen VD, Follansbee WP, Conte CG, et al. Thallium perfusion defects predict subsequent cardiac dysfunction in patients with systemic sclerosis. Arthritis Rheum 1996; 39: 677-681. PMID: 8630120

243. Fox BD, Shimony A, Langleben D, et al. High prevalence of occult left heart disease in scleroderma-pulmonary hypertension. Eur Respir J 2013; 42: 1083-1091. PMID: 23258775

244. Yoshida S, Treatment guidelines for different types of MCTD: Pulmonary hypertension. Taskforce to clarify the pathophysiology of and establish the treatment for mixed connective tissue disease. Guidelines for the treatment of mixed connective tissue disease (3rd revision) 2011: 27-32. [in Japanese]

245. Sanchez O, Sitbon O, Jaiis X, et al. Immunosuppressive therapy in connective tissue diseases-associated pulmonary arterial hypertension. Chest 2006; 130: 182-189. PMID: 16840400

246. Jais X, Launay D, Yaici A, et al. Immunosuppressive therapy in lupus- and mixed connective tissue disease-associated pulmonary arterial hypertension: A retrospective analysis of twentythree cases. Arthritis Rheum 2008; 58: 521-531. PMID: 18240255

247. Miyamichi-Yamamoto S, Fukumoto Y, Sugimura K, et al. Intensive immunosuppressive therapy improves pulmonary hemodynamics and long-term prognosis in patients with pulmonary arterial hypertension associated with connective tissue disease. Circ J 2011; 75: 2668-2674. PMID: 21873802

248. Kato M, Kataoka H, Odani T, et al. The short-term role of corticosteroid therapy for pulmonary arterial hypertension associated with connective tissue diseases: Report of five cases and a literature review. Lupus 2011; 20: 1047-1056. PMID: 21676917

249. Badesch DB, Tapson VF, McGoon MD, et al. Continuous intravenous epoprostenol for pulmonary hypertension due to the scleroderma spectrum of disease: A randomized, controlled trial. Ann Intern Med 2000; 132: 425-434. PMID: 10733441

250. Shirai Y, Yasuoka H, Takeuchi T, et al. Intravenous epoprostenol treatment of patients with connective tissue disease and pulmonary arterial hypertension at a single center. Mod Rheumatol 2013; 23: 1211-1220. PMID: 23359006

251. Rubin LJ, Badesch DB, Barst RJ, et al. Bosentan therapy for pulmonary arterial hypertension. N Engl J Med 2002; 346: 896-903. PMID: 11907289

252. Ghofrani HA, Galiè N, Grimminger F, et al. PATENT-1 Study Group. Riociguat for the treatment of pulmonary arterial hypertension. $N$ Engl $J$ Med 2013; 369: 330-340. PMID: 23883378

253. Badesch DB, Hill NS, Burgess G, et al. SUPER Study Group. Sildenafil for pulmonary arterial hypertension associated with connective tissue disease. $J$ Rheumatol 2007; 34: 2417-2422. PMID: 17985403

254. Coghlan JG, Galiè N, Barberà JA, et al. AMBITION investigators. Initial combination therapy with ambrisentan and tadalafil in connective tissue disease-associated pulmonary arterial hypertension (CTD-PAH): Subgroup analysis from the AMBITION trial. Ann Rheum Dis 2017; 76: 1219-1227. PMID: 28039187

255. Shirai Y, Kuwana M. Complex pathophysiology of pulmonary hypertension associated with systemic sclerosis: Potential unfavorable effects of vasodilators. $J$ Scleroderma Relat Disord 2017; 2: 92-99.

256. Humbert M, Coghlan JG, Ghofrani HA, et al. Riociguat for the treatment of pulmonary arterial hypertension associated with connective tissue disease: Results from PATENT-1 and PATENT-2. Ann Rheum Dis 2017; 76: 422-426. PMID: 27457511

257. Hoeper MM, McLaughlin VV, Barberá JA, et al. Initial combination therapy with ambrisentan and tadalafil and mortality in patients with pulmonary arterial hypertension: A secondary analysis of the results from the randomised, controlled AMBITION study. Lancet Respir Med 2016; 4: 894-901. PMID: 27745818

258. Krowka MJ, Fallon MB, Kawut SM, et al. International Liver Transplant Society Practice Guidelines: Diagnosis and management of hepatopulmonary syndrome and portopulmonary hypertension. Transplantation 2016; 100: 1440-1452. PMID: 27326810

259. Rodríguez-Roisin R, Krowka MJ, Hervé P, et al. ERS Task Force Pulmonary-Hepatic Vascular Disorders (PHD) Scientific Committee. Pulmonary-Hepatic vascular Disorders (PHD). Eur Respir J 2004; 24: 861-880. PMID: 15516683

260. Hadengue A, Benhayoun MK, Lebrec D, et al. Pulmonary hypertension complicating portal hypertension: Prevalence and 
relation to splanchnic hemodynamics. Gastroenterology 1991; 100: 520-528. PMID: 1985048

261. Zopey R, Susanto I, Barjaktarevic I, et al. Transition from hepatopulmonary syndrome to portopulmonary hypertension: A case series of 3 patients. Case Rep Pulmonol 2013; 2013: 561870. PMID: 24324910

262. Kawut SM, Taichman DB, Ahya VN, et al. Hemodynamics and survival of patients with portopulmonary hypertension. Liver Transpl 2005; 11: 1107-1111. PMID: 16123953

263. Roberts KE, Fallon MB, Krowka MJ, et al. Pulmonary Vascular Complications of Liver Disease Study Group. Genetic risk factors for portopulmonary hypertension in patients with advanced liver disease. Am J Respir Crit Care Med 2009; 179: 835-842. PMID: 19218192

264. Krowka MJ, Miller DP, Barst RJ, et al. Portopulmonary hypertension: A report from the US-based REVEAL Registry. Chest 2012; 141: 906-915. PMID: 21778257

265. Swanson KL, Wiesner RH, Nyberg SL, et al. Survival in portopulmonary hypertension: Mayo Clinic experience categorized by treatment subgroups. Am J Transplant 2008; 8: 2445-2453. PMID: 18782292

266. Austin MJ, McDougall NI, Wendon JA, et al. Safety and efficacy of combined use of sildenafil, bosentan, and iloprost before and after liver transplantation in severe portopulmonary hypertension. Liver Transpl 2008; 14: 287-291. PMID: 18306330

267. Porres-Aguilar M, Zuckerman MJ, Figueroa-Casas JB, et al. Portopulmonary hypertension: State of the art. Ann Hepatol 2008; 7: 321 - 330. PMID: 19034231

268. Saleemi S. Portopulmonary hypertension. Ann Thorac Med 2010; 5: 5-9. PMID: 20351954

269. Hoeper MM, Krowka MJ, Strassburg CP. Portopulmonary hypertension and hepatopulmonary syndrome. Lancet 2004; 363: $1461-1468$. PMID: 15121411

270. Robalino BD, Moodie DS. Association between primary pulmonary hypertension and portal hypertension: Analysis of its pathophysiology and clinical, laboratory and hemodynamic manifestations. J Am Coll Cardiol 1991; 17: 492-498. PMID: 1991908

271. Provencher S, Herve P, Jais X, et al. Deleterious effects of betablockers on exercise capacity and hemodynamics in patients with portopulmonary hypertension. Gastroenterology 2006; 130: $120-126$. PMID: 16401475

272. Hoeper MM, Seyfarth HJ, Hoeffken G, et al. Experience with inhaled iloprost and bosentan in portopulmonary hypertension. Eur Respir J 2007; 30: 1096-1102. PMID: 17652314

273. Kuo PC, Johnson LB, Plotkin JS, et al. Continuous intravenous infusion of epoprostenol for the treatment of portopulmonary hypertension. Transplantation 1997; 63: 604-606. PMID: 9047158

274. Reichenberger F, Voswinckel R, Steveling E, et al. Sildenafil treatment for portopulmonary hypertension. Eur Respir $J$ 2006; 28: $563-567$. PMID: 16807265

275. Halank M, Knudsen L, Seyfarth HJ, et al. Ambrisentan improves exercise capacity and symptoms in patients with portopulmonary hypertension. Z Gastroenterol 2011; 49: 1258 1262. PMID: 21887662

276. Benjaminov FS, Prentice M, Sniderman KW, et al. Portopulmonary hypertension in decompensated cirrhosis with refractory ascites. Gut 2003; 52: 1355-1362. PMID: 12912870

277. Cartin-Ceba R, Swanson K, Iyer V, et al. Safety and efficacy of ambrisentan for the treatment of portopulmonary hypertension. Chest 2011; 139: 109-114. PMID: 20705798

278. Krowka MJ, Plevak DJ, Findlay JY, et al. Pulmonary hemodynamics and perioperative cardiopulmonary-related mortality in patients with portopulmonary hypertension undergoing liver transplantation. Liver Transpl 2000; 6: 443-450. PMID: 10915166

279. Raevens S, De Pauw M, Reyntjens K, et al. Oral vasodilator therapy in patients with moderate to severe portopulmonary hypertension as a bridge to liver transplantation. Eur $J$ Gastroenterol Hepatol 2013; 25: 495-502. PMID: 23242127

280. Plotkin JS, Kuo PC, Rubin LJ, et al. Successful use of chronic epoprostenol as a bridge to liver transplantation in severe portopulmonary hypertension. Transplantation 1998; 65: $457-$ 459. PMID: 9500616

281. Galiè N, Corris PA, Frost A, et al. Updated treatment algorithm of pulmonary arterial hypertension. J Am Coll Cardiol 2013; 62: D60-D72. PMID: 24355643

282. Shiina Y, Toyoda T, Kawasoe Y, et al. Prevalence of adult patients with congenital heart disease in Japan. Int J Cardiol
2011; 146: 13-16. PMID: 19493578

283. Engelfriet PM, Duffels MG, Möller T, et al. Pulmonary arterial hypertension in adults born with a heart septal defect: The Euro Heart Survey on adult congenital heart disease. Heart 2007; 93: 682-687. PMID: 17164490

284. van Riel AC, Schuuring MJ, van Hessen ID, et al. Contemporary prevalence of pulmonary arterial hypertension in adult congenital heart disease following the updated clinical classification. Int J Cardiol 2014; 174: 299-305. PMID: 24794056

285. Barst RJ, Ivy DD, Foreman AJ, et al. Four- and seven-year outcomes of patients with congenital heart disease-associated pulmonary arterial hypertension (from the REVEAL Registry). Am J Cardiol 2014; 113: 147-155. PMID: 24176071

286. Galiè N, Beghetti M, Gatzoulis MA, et al. Bosentan Randomized Trial of Endothelin Antagonist Therapy-5 (BREATHE-5) Investigators. Bosentan therapy in patients with Eisenmenger syndrome: A multicenter, double-blind, randomized, placebocontrolled study. Circulation 2006; 114: 48-54. PMID: 16801459

287. Kuijpers JM, Mulder BJ, Bouma BJ. Secundum atrial septal defect in adults: A practical review and recent developments. Neth Heart J 2015; 23: 205-211. PMID: 25884091

288. Dimopoulos K, Inuzuka R, Goletto S, et al. Improved survival among patients with Eisenmenger syndrome receiving advanced therapy for pulmonary arterial hypertension. Circulation 2010; 121: 20-25. PMID: 20026774

289. Cha KS, Cho KI, Seo JS, et al. Effects of inhaled iloprost on exercise capacity, quality of life, and cardiac function in patients with pulmonary arterial hypertension secondary to congenital heart disease (the Eisenmenger syndrome) (from the EIGER Study). Am J Cardiol 2013; 112: 1834-1839. PMID: 24012036

290. Yang SI, Chung WJ, Jung SH, et al. Effects of inhaled iloprost on congenital heart disease with Eisenmenger syndrome. Pediatr Cardiol 2012; 33: 744-748. PMID: 22349672

291. Diller GP, Körten MA, Bauer UM, et al. German Competence Network for Congenital Heart Defects Investigators. Current therapy and outcome of Eisenmenger syndrome: Data of the German National Register for congenital heart defects. Eur Heart J 2016; 37: 1449-1455. PMID: 26843280

292. Manes A, Palazzini M, Leci E, et al. Current era survival of patients with pulmonary arterial hypertension associated with congenital heart disease: A comparison between clinical subgroups. Eur Heart J 2014; 35: 716-724. PMID: 23455361

293. Kijima Y, Akagi T, Takaya Y, et al. Treat and repair strategy in patients with atrial septal defect and significant pulmonary arterial hypertension. Circ $J$ 2016; 80: 227-234. PMID: 26567484

294. Izquierdo MT, Almenar L, Morales P, et al. Mortality after heart-lung transplantation experience in a reference center. Transplant Proc 2007; 39: 2360-2361. PMID: 17889189

295. Goerler H, Simon A, Gohrbandt B, et al. Heart-lung and lung transplantation in grown-up congenital heart disease: Long-term single centre experience. Eur J Cardiothorac Surg 2007; 32: 926-931. PMID: 17900918

296. Waddell TK, Bennett L, Kennedy R, et al. Heart-lung or lung transplantation for Eisenmenger syndrome. J Heart Lung Transplant 2002; 21: 731-737. PMID: 12100899

297. Stoica SC, McNeil KD, Perreas K, et al. Heart-lung transplantation for Eisenmenger syndrome: Early and long-term results. Ann Thorac Surg 2001; 72: 1887-1891. PMID: 11789765

298. Kay JM, Smith P, Heath D. Aminorex and the pulmonary circulation. Thorax 1971; 26: 262-270. PMID: 5089490

299. Simonneau G, Fartoukh M, Sitbon O, et al. Primary pulmonary hypertension associated with the use of fenfluramine derivatives. Chest 1998; 114: 195S-199S. PMID: 9741568

300. Souza R, Humbert M, Sztrymf B, et al. Pulmonary arterial hypertension associated with fenfluramine exposure: Report of 109 cases. Eur Respir J 2008; 31: 343 -348. PMID: 17959632

301. Savale L, Chaumais MC, Cottin V, et al. Pulmonary hypertension associated with benfluorex exposure. Eur Respir J 2012; 40: 1164-1172. PMID: 22523367

302. Chin KM, Channick RN, Rubin LJ. Is methamphetamine use associated with idiopathic pulmonary arterial hypertension? Chest 2006; 130: 1657-1663. PMID: 17166979

303. Dhillon S, Kaker A, Dosanjh A, et al. Irreversible pulmonary hypertension associated with the use of interferon alpha for chronic hepatitis C. Dig Dis Sci 2010; 55: 1785-1790. PMID: 20411421

304. Caravita S, Secchi MB, Wu SC, et al. Sildenafil therapy for interferon- $\beta$-1a-induced pulmonary arterial hypertension: A case report. Cardiology 2011; 120: 187-189. PMID: 22269997 
305. Kieler H, Artama M, Engeland A, et al. Selective serotonin reuptake inhibitors during pregnancy and risk of persistent pulmonary hypertension in the newborn: Population based cohort study from the five Nordic countries. BMJ 2012; 344: d8012. PMID: 22240235

306. Montani D, Bergot E, Günther S, et al. Pulmonary arterial hypertension in patients treated by dasatinib. Circulation 2012; 125: 2128 -2137. PMID: 22451584

307. Quilot FM, Georges M, Favrolt N, et al. Pulmonary hypertension associated with ponatinib therapy. Eur Respir J 2016; 47: 676-679. PMID: 26743481

308. The Compliance and Narcotics Division and the Inspection and Safety Division of the Pharmaceutical Safety and Environmental Health Bureau, MHLW. Health hazards (suspected cases) due to the use of plant products (December 27, 2016). http://www. mhlw.go.jp/file/06-Seisakujouhou-11130500-Shokuhinanzenbu/ 0000147453.pdf [in Japanese]

309. Kanmogne GD, Primeaux C, Grammas P. Induction of apoptosis and endothelin-1 secretion in primary human lung endothelial cells by HIV-1 gp120 proteins. Biochem Biophys Res Commun 2005; 333: 1107-1115. PMID: 15979050

310. Petitpretz P, Brenot F, Azarian R, et al. Pulmonary hypertension in patients with human immunodeficiency virus infection: Comparison with primary pulmonary hypertension. Circulation 1994; 89: 2722-2727. PMID: 8205687

311. Degano B, Guillaume M, Savale L, et al. HIV-associated pulmonary arterial hypertension: Survival and prognostic factors in the modern therapeutic era. AIDS 2010; 24: 67-75. PMID: 19770696

312. Sitbon O, Yaici A, Cottin V, et al. The changing picture of patients with pulmonary arterial hypertension in France. Eur Heart J 2011; 32: 675-676.

313. Montani D, Savale L, Natali D, et al. Long-term response to calcium-channel blockers in non-idiopathic pulmonary arterial hypertension. Eur Heart $J$ 2010; 31: 1898-1907. PMID: 20543192

314. Sitbon O, Gressin V, Speich R, et al. Bosentan for the treatment of human immunodeficiency virus-associated pulmonary arterial hypertension. Am J Respir Crit Care Med 2004; 170: 1212-1217. PMID: 15317666

315. Schumacher YO, Zdebik A, Huonker M, et al. Sildenafil in HIV-related pulmonary hypertension. AIDS 2001; 15: $1747-$ 1748. PMID: 11546958

316. Ghofrani HA, Friese G, Discher T, et al. Inhaled iloprost is a potent acute pulmonary vasodilator in HIV-related severe pulmonary hypertension. Eur Respir J 2004; 23: 321-326. PMID: 14979511

317. Nunes H, Humbert M, Sitbon O, et al. Prognostic factors for survival in human immunodeficiency virus-associated pulmonary arterial hypertension. Am J Respir Crit Care Med 2003; 167: 1433-1439. PMID: 12615632

318. Zuber JP, Calmy A, Evison JM, et al. Swiss HIV Cohort Study Group. Pulmonary arterial hypertension related to HIV infection: Improved hemodynamics and survival associated with antiretroviral therapy. Clin Infect Dis 2004; 38: 1178-1185. PMID: 15095226

319. Opravil M, Pechère $M$, Speich R, et al. HIV-associated primary pulmonary hypertension. A case control study. Swiss HIV Cohort Study. Am J Respir Crit Care Med 1997; 155: 990-995. PMID: 9117037

320. Pugliese A, Isnardi D, Saini A, et al. Impact of highly active antiretroviral therapy in HIV-positive patients with cardiac involvement. J Infect 2000; 40: 282-284. PMID: 10908024

321. Mandel J, Mark EJ, Hales CA. Pulmonary veno-occlusive disease. Am J Respir Crit Care Med 2000; 162: 1964-1973. PMID: 11069841

322. Lantuéjoul S, Sheppard MN, Corrin B, et al. Pulmonary venoocclusive disease and pulmonary capillary hemangiomatosis: A clinicopathologic study of 35 cases. Am J Surg Pathol 2006; 30: 850-857. PMID: 16819327

323. Holcomb BW, Loyd JE, Ely EW, et al. Pulmonary venoocclusive disease: a case series and new observations. Chest 2000; 118: 1671 -1679. PMID: 11115457

324. Almagro $\mathrm{P}$, Julià $\mathrm{J}$, Sanjaume M, et al. Pulmonary capillary hemangiomatosis associated with primary pulmonary hypertension: Report of 2 new cases and review of 35 cases from the literature. Medicine (Baltimore) 2002; 81: 417-424. PMID: 12441898

325. Eyries M, Montani D, Girerd B, et al. EIF2AK4 mutations cause pulmonary veno-occlusive disease, a recessive form of pulmonary hypertension. Nat Genet 2014; 46: 65-69. PMID: 24292273

326. Best DH, Sumner KL, Austin ED, et al. EIF2AK4 mutations in pulmonary capillary hemangiomatosis. Chest 2014; 145: 231 -236. PMID: 24135949

327. Ranchoux B, Günther S, Quarck R, et al. Chemotherapyinduced pulmonary hypertension: Role of alkylating agents. Am J Pathol 2015; 185: 356-371. PMID: 25497573

328. Perros F, Günther S, Ranchoux B, et al. Mitomycin-induced pulmonary veno-occlusive disease: Evidence from human disease and animal models. Circulation 2015; 132: 834-847. PMID: 26130118

329. Ishibashi-Ueda H. Pathology of pulmonary veno-occlusive disease. The Japanese Journal of Chest Diseases 2014; 73: 289 299. [in Japanese]

330. Ogawa A, Miyaji K, Yamadori I, et al. Safety and efficacy of epoprostenol therapy in pulmonary veno-occlusive disease and pulmonary capillary hemangiomatosis. Circ J 2012; 76: 1729-1736. PMID: 22481098

331. Miura A, Nakamura K, Kusano KF, et al. Three-dimensional structure of pulmonary capillary vessels in patients with pulmonary hypertension. Circulation 2010; 121: 2151-2153. PMID: 20479166

332. Montani D, Jaiis X, Price LC, et al. Cautious epoprostenol therapy is a safe bridge to lung transplantation in pulmonary veno-occlusive disease. Eur Respir J 2009; 34: 1348-1356. PMID: 19541723

333. Abramson SV, Burke JF, Kelly JJ, et al. Pulmonary hypertension predicts mortality and morbidity in patients with dilated cardiomyopathy. Ann Intern Med 1992; 116: 888-895. PMID: 1580444

334. Ghio S, Gavazzi A, Campana C, et al. Independent and additive prognostic value of right ventricular systolic function and pulmonary artery pressure in patients with chronic heart failure. J Am Coll Cardiol 2001; 37: 183-188. PMID: 11153735

335. Lam CS, Roger VL, Rodeheffer RJ, et al. Pulmonary hypertension in heart failure with preserved ejection fraction: A communitybased study. J Am Coll Cardiol 2009; 53: 1119-1126. PMID: 19324256

336. Tatebe S, Fukumoto Y, Sugimura K, et al. Clinical significance of reactive post-capillary pulmonary hypertension in patients with left heart disease. Circ J 2012; 76: 1235-1244. PMID: 22313804

337. Ibe T, Wada H, Sakakura K, et al. Pulmonary hypertension due to left heart disease: The prognostic implications of diastolic pulmonary vascular pressure gradient. J Cardiol 2016; 67: 555-559. PMID: 26299611

338. Leung CC, Moondra V, Catherwood E, et al. Prevalence and risk factors of pulmonary hypertension in patients with elevated pulmonary venous pressure and preserved ejection fraction. $\mathrm{Am}$ J Cardiol 2010; 106: 284-286. PMID: 20599017

339. Kjaergaard J, Akkan D, Iversen KK, et al. Prognostic importance of pulmonary hypertension in patients with heart failure. Am J Cardiol 2007; 99: 1146-1150. PMID: 17437745

340. Plazak W, Zabinska-Plazak E, Wojas-Pelc A, et al. Heart structure and function in systemic sclerosis. Eur $J$ Dermatol 2002; 12: 257-262. PMID: 11978567

341. Dupont M, Mullens W, Skouri HN, et al. Prognostic role of pulmonary arterial capacitance in advanced heart failure. Circ Heart Fail 2012; 5: 778-785. PMID: 23087402

342. Dragu R, Rispler S, Habib M, et al. Pulmonary arterial capacitance in patients with heart failure and reactive pulmonary hypertension. Eur J Heart Fail 2015; 17: 74-80. PMID: 25388783

343. Sugimoto K, Yoshihisa A, Nakazato K, et al. Pulmonary arterial capacitance predicts cardiac events in pulmonary hypertension due to left heart disease. PLoS One 2016; 11: e0165603. PMID: 27875533

344. Al-Naamani N, Preston IR, Paulus JK, et al. Pulmonary arterial capacitance is an importantpredictor of mortality in heart failure with a preserved ejection fraction. JACC Heart Fail 2015; 3: 467-474. PMID: 26046840

345. Califf RM, Adams KF, McKenna WJ, et al. A randomized controlled trial of epoprostenol therapy for severe congestive heart failure: The Flolan International Randomized Survival Trial (FIRST). Am Heart J 1997; 134: 44-54. PMID: 9266782

346. Kalra PR, Moon JC, Coats AJ. Do results of the ENABLE (Endothelin Antagonist Bosentan for Lowering Cardiac Events in Heart Failure) study spell the end for non-selective endothelin antagonism in heart failure? Int J Cardiol 2002; 85: 195-197. PMID: 12208583

347. Clinical study to evaluate the safety and tolerability of 
macitentan in subjects with combined pre- and post-capillary pulmonary hypertension $(\mathrm{CpcPH})$ due to left ventricular dysfunction (MELODY-1). https://clinicaltrials.gov/ct2/show/ NCT02070991

348. Guazzi M, Vicenzi M, Arena R, et al. Pulmonary hypertension in heart failure with preserved ejection fraction: A target of phosphodiesterase-5 inhibition in a 1-year study. Circulation 2011; 124: 164-174. PMID: 21709061

349. Redfield MM, Chen HH, Borlaug BA, et al. RELAX Trial. Effect of phosphodiesterase-5 inhibition on exercise capacity and clinical status in heart failure with preserved ejection fraction: A randomized clinical trial. JAMA 2013; 309: 1268-1277. PMID: 23478662

350. Hoendermis ES, Liu LC, Hummel YM, et al. Effects of sildenafil on invasive haemodynamics and exercise capacity in heart failure patients with preserved ejection fraction and pulmonary hypertension: A randomized controlled trial. Eur Heart J 2015; 36: $2565-2573$. PMID: 26188003

351. Andersen KH, Iversen M, Kjaergaard J, et al. Prevalence, predictors, and survival in pulmonary hypertension related to end-stage chronic obstructive pulmonary disease. $J$ Heart Lung Transplant 2012; 31: 373-380. PMID: 22226804

352. Carlsen J, Hasseriis Andersen K, Boesgaard S, et al. Pulmonary arterial lesions in explanted lungs after transplantation correlate with severity of pulmonary hypertension in chronic obstructive pulmonary disease. $J$ Heart Lung Transplant 2013; 32: 347-354. PMID: 23265910

353. Weitzenblum E, Sautegeau A, Ehrhart M, et al. Long-term course of pulmonary arterial pressure in chronic obstructive pulmonary disease. Am Rev Respir Dis 1984; 130: 993-998. PMID: 6439091

354. Burrows B, Kettel LJ, Niden AH, et al. Patterns of cardiovascular dysfunction in chronic obstructive lung disease. $N$ Engl $J$ Med 1972; 286: 912-918. PMID: 5013974

355. Oswald-Mammosser M, Weitzenblum E, Quoix E, et al. Prognostic factors in COPD patients receiving long-term oxygen therapy: Importance of pulmonary artery pressure. Chest 1995; 107: 1193 -1198. PMID: 7750305

356. Arcasoy SM, Christie JD, Ferrari VA, et al. Echocardiographic assessment of pulmonary hypertension in patients with advanced lung disease. Am J Respir Crit Care Med 2003; 167: 735-740. PMID: 12480614

357. Fisher MR, Criner GJ, Fishman AP, et al. NETT Research Group. Estimating pulmonary artery pressures by echocardiography in patients with emphysema. Eur Respir J 2007; 30: 914-921. PMID: 17652313

358. Leuchte HH, Baumgartner RA, Nounou ME, et al. Brain natriuretic peptide is a prognostic parameter in chronic lung disease. Am J Respir Crit Care Med 2006; 173: 744-750. PMID: 16415273

359. Boerrigter BG, Bogaard HJ, Trip P, et al. Ventilatory and cardiocirculatory exercise profiles in COPD: The role of pulmonary hypertension. Chest 2012; 142: 1166-1174. PMID: 22556320

360. Weinmann GG, Chiang YP, Sheingold S. The National Emphysema Treatment Trial (NETT): A study in agency collaboration. Proc Am Thorac Soc 2008; 5: 381-384. PMID: 18453343

361. Horn M, Ries A, Neveu C, et al. Restrictive ventilatory pattern in precapillary pulmonary hypertension. Am Rev Respir Dis 1983; 128: 163-165. PMID: 6870057

362. Burke CM, Glanville AR, Morris AJ, et al. Pulmonary function in advanced pulmonary hypertension. Thorax 1987; 42: $131-$ 135. PMID: 3433237

363. Spiekerkoetter E, Fabel H, Hoeper MM. Effects of inhaled salbutamol in primary pulmonary hypertension. Eur Respir $J$ 2002; 20: 524-528. PMID: 12358324

364. Meyer FJ, Ewert R, Hoeper MM, et al. German PPH Study Group. Peripheral airway obstruction in primary pulmonary hypertension. Thorax 2002; 57: 473-476. PMID: 12037220

365. Seeger W, Adir Y, Barberà JA, et al. Pulmonary hypertension in chronic lung diseases. J Am Coll Cardiol 2013; 62: D109D116. PMID: 24355635

366. Albert RK, Au DH, Blackford AL, et al. Long-Term Oxygen Treatment Trial Research Group. A randomized trial of longterm oxygen for COPD with moderate desaturation. $N$ Engl J Med 2016; 375: 1617-1627. PMID: 27783918

367. Barberà JA, Blanco I. Pulmonary hypertension in patients with chronic obstructive pulmonary disease: Advances in pathophysiology and management. Drugs 2009; 69: 1153-1171. PMID: 19537834
368. Boeck L, Tamm M, Grendelmeier P, et al. Acute effects of aerosolized iloprost in COPD related pulmonary hypertension: A randomized controlled crossover trial. PLoS One 2012; 7: e52248. PMID: 23300624

369. Stolz D, Rasch H, Linka A, et al. A randomised, controlled trial of bosentan in severe COPD. Eur Respir J 2008; 32: 619-628. PMID: 18448495

370. Valerio G, Bracciale P, Grazia D'Agostino A. Effect of bosentan upon pulmonary hypertension in chronic obstructive pulmonary disease. Ther Adv Respir Dis 2009; 3: 15-21. PMID: 19293199

371. Blanco I, Gimeno E, Munoz PA, et al. Hemodynamic and gas exchange effects of sildenafil in patients with chronic obstructive pulmonary disease and pulmonary hypertension. Am J Respir Crit Care Med 2010; 181: 270-278. PMID: 19875684

372. Rietema H, Holverda S, Bogaard HJ, et al. Sildenafil treatment in COPD does not affect stroke volume or exercise capacity. Eur Respir J 2008; 31: 759-764. PMID: 18094009

373. Lederer DJ, Bartels MN, Schluger NW, et al. Sildenafil for chronic obstructive pulmonary disease: A randomized crossover trial. COPD 2012; 9: 268-275. PMID: 22360383

374. Blanco I, Santos S, Gea J, et al. Sildenafil to improve respiratory rehabilitation outcomes in COPD: A controlled trial. Eur Respir J 2013; 42: 982-992. PMID: 23429918

375. Rao RS, Singh S, Sharma BB, et al. Sildenafil improves sixminute walk distance in chronic obstructive pulmonary disease: A randomised, double-blind, placebo-controlled trial. Indian $J$ Chest Dis Allied Sci 2011; 53: 81-85. PMID: 21545068

376. Hurdman J, Condliffe R, Elliot CA, et al. Pulmonary hypertension in COPD: Results from the ASPIRE registry. Eur Respir $J$ 2013; 41: 1292-1301. PMID: 23018917

377. Brewis MJ, Church AC, Johnson MK, et al. Severe pulmonary hypertension in lung disease: Phenotypes and response to treatment. Eur Respir J 2015; 46: 1378-1389. PMID: 26293503

378. Fuehner T, Kuehn C, Hadem J, et al. Extracorporeal membrane oxygenation in awake patients as bridge to lung transplantation. Am J Respir Crit Care Med 2012; 185: 763-768. PMID: 22268135

379. Hamada K, Nagai S, Tanaka S, et al. Significance of pulmonary arterial pressure and diffusion capacity of the lung as prognosticator in patients with idiopathic pulmonary fibrosis. Chest 2007; 131: 650-656. PMID: 17317730

380. Kimura M, Taniguchi H, Kondoh Y, et al. Pulmonary hypertension as a prognostic indicator at the initial evaluation in idiopathic pulmonary fibrosis. Respiration $2013 ; \mathbf{8 5}$ : 456-463. PMID: 23257350

381. Behr J, Ryu JH. Pulmonary hypertension in interstitial lung disease. Eur Respir J 2008; 31: 1357-1367. PMID: 18515559

382. Minai OA, Santacruz JF, Alster JM, et al. Impact of pulmonary hemodynamics on 6-min walk test in idiopathic pulmonary fibrosis. Respir Med 2012; 106: 1613-1621. PMID: 22902266

383. Nathan SD, Shlobin OA, Ahmad S, et al. Serial development of pulmonary hypertension in patients with idiopathic pulmonary fibrosis. Respiration 2008; 76: 288-294. PMID: 18216461

384. Shorr AF, Wainright JL, Cors CS, et al. Pulmonary hypertension in patients with pulmonary fibrosis awaiting lung transplant. Eur Respir J 2007; 30: 715-721. PMID: 17626111

385. Nathan SD, Shlobin OA, Ahmad S, et al. Pulmonary hypertension and pulmonary function testing in idiopathic pulmonary fibrosis. Chest 2007; 131: 657-663. PMID: 17356077

386. Tanabe N, Taniguchi H, Tsujino I, et al. Japanese Respiratory Society Lung Disease Pulmonary Hypertension Study Group. Current trends in the management of pulmonary hypertension associated with respiratory disease in institutions approved by the Japanese Respiratory Society. Respir Investig 2014; 52: 167-172. PMID: 24853016

387. Lettieri CJ, Nathan SD, Barnett SD, et al. Prevalence and outcomes of pulmonary arterial hypertension in advanced idiopathic pulmonary fibrosis. Chest 2006; 129: 746-752. PMID: 16537877

388. King TE Jr, Tooze JA, Schwarz MI, et al. Predicting survival in idiopathic pulmonary fibrosis: Scoring system and survival model. Am J Respir Crit Care Med 2001; 164: 1171-1181. PMID: 11673205

389. Nadrous HF, Pellikka PA, Krowka MJ, et al. Pulmonary hypertension in patients with idiopathic pulmonary fibrosis. Chest 2005; 128: 2393-2399. PMID: 16236900

390. Tanabe N, Taniguchi H, Tsujino I, et al. JRS Lung Disease PH Study Group. Multi-institutional retrospective cohort study of patients with severe pulmonary hypertension associated with respiratory diseases. Respirology 2015; 20: 805-812. PMID: 


\section{4}

391. Weitzenblum E, Ehrhart M, Rasaholinjanahary J, et al. Pulmonary hemodynamics in idiopathic pulmonary fibrosis and other interstitial pulmonary diseases. Respiration 1983; 44: 118-127. PMID: 6836187

392. Renzoni EA, Walsh DA, Salmon M, et al. Interstitial vascularity in fibrosing alveolitis. Am J Respir Crit Care Med 2003; 167: 438-443. PMID: 12406847

393. Sturani C, Papiris S, Galavotti V, et al. Pulmonary vascular responsiveness at rest and during exercise in idiopathic pulmonary fibrosis: Effects of oxygen and nifedipine. Respiration 1986; 50: $117-129$. PMID: 3749613

394. Colombat M, Mal H, Groussard O, et al. Pulmonary vascular lesions in end-stage idiopathic pulmonary fibrosis: Histopathologic study on lung explant specimens and correlations with pulmonary hemodynamics. Hum Pathol 2007; 38: 60-65. PMID: 16949908

395. Nathan SD, Shlobin OA, Barnett SD, et al. Right ventricular systolic pressure by echocardiography as a predictor of pulmonary hypertension in idiopathic pulmonary fibrosis. Respir Med 2008; 102: 1305-1310. PMID: 18619825

396. Boutou AK, Pitsiou GG, Trigonis I, et al. Exercise capacity in idiopathic pulmonary fibrosis: The effect of pulmonary hypertension. Respirology 2011; 16: 451 - 458. PMID: 21122030

397. Corte TJ, Keir GJ, Dimopoulos K, et al. BPHIT Study Group. Bosentan in pulmonary hypertension associated with fibrotic idiopathic interstitial pneumonia. Am J Respir Crit Care Med 2014; 190: 208-217. PMID: 24937643

398. Zisman DA, Schwarz M, Anstrom KJ, et al. Idiopathic Pulmonary Fibrosis Clinical Research Network. A controlled trial of sildenafil in advanced idiopathic pulmonary fibrosis. $N$ Engl $J$ Med 2010; 363: 620-628. PMID: 20484178

399. Han MK, Bach DS, Hagan PG, et al. IPFnet Investigators. Sildenafil preserves exercise capacity in patients with idiopathic pulmonary fibrosis and right-sided ventricular dysfunction. Chest 2013; 143: 1699-1708. PMID: 23732584

400. King TE Jr, Behr J, Brown KK, et al. BUILD-1: A randomized placebo-controlled trial of bosentan in idiopathic pulmonary fibrosis. Am J Respir Crit Care Med 2008; 177: 75-81. PMID: 17901413

401. King TE Jr, Brown KK, Raghu G, et al. BUILD-3: A randomized, controlled trial of bosentan in idiopathic pulmonary fibrosis. Am J Respir Crit Care Med 2011; 184: 92-99. PMID: 21474646

402. Raghu G, Behr J, Brown KK, et al. ARTEMIS-IPF Investigators. Treatment of idiopathic pulmonary fibrosis with ambrisentan: A parallel, randomized trial. Ann Intern Med 2013; 158: 641-649. PMID: 23648946

403. Raghu G, Million-Rousseau R, Morganti A, et al. MUSIC Study Group. Macitentan for the treatment of idiopathic pulmonary fibrosis: The randomised controlled MUSIC trial. Eur Respir J 2013; 42: 1622-1632. PMID: 23682110

404. Teramachi R, Taniguchi H, Kondoh Y, et al. Progression of mean pulmonary arterial pressure in idiopathic pulmonary fibrosis with mild to moderate restriction. Respirology 2017; 22: 986-990. PMID: 28117530

405. Cottin V, Le Pavec J, Prévot G, et al. GERM"O"P. Pulmonary hypertension in patients with combined pulmonary fibrosis and emphysema syndrome. Eur Respir J 2010; 35: 105-111. PMID: 19643948

406. Sato T, Tsujino I, Tanino M, et al. Broad and heterogeneous vasculopathy in pulmonary fibrosis and emphysema with pulmonary hypertension. Respirol Case Rep 2013; 1: 10-13. PMID: 25473528

407. Mercurio V, Carlomagno G, Fazio S. Response to pulmonary vasodilator treatment in a former smoker with combined interstitial lung disease complicated by pulmonary hypertension: Case report and review of the literature. Heart Lung 2012; 41: 512-517. PMID: 22054725

408. Cottin V. Clinical case: Combined pulmonary fibrosis and emphysema with pulmonary hypertension--clinical management. BMC Res Notes 2013; 6: S2. PMID: 23734851

409. Sato T, Tsujino I, Sugimoto A, et al. The effects of pulmonary vasodilating agents on right ventricular parameters in severe group 3 pulmonary hypertension: A pilot study. Pulm Circ 2016; 6: 524-531. PMID: 28090294

410. Tatsumi K. A research study on respiratory failure. The 2014 summary and project report of the study group on respiratory failure in the Health and Labor Sciences Research Grant for intractable diseases 2015: 48-51. [in Japanese]

411. Weese-Mayer DE, Silvestri JM, Menzies LJ, et al. Congenital central hypoventilation syndrome: Diagnosis, management, and long-term outcome in thirty-two children. J Pediatr 1992; 120: 381 -387. PMID: 1538284

412. Idiopathic congenital central hypoventilation syndrome: Diagnosis and management. American Thoracic Society. Am $J$ Respir Crit Care Med 1999; 160: 368 -373. PMID: 10390427

413. Diep B, Wang A, Kun S, et al. Diaphragm pacing without tracheostomy in congenital central hypoventilation syndrome patients. Respiration 2015; 89: 534-538. PMID: 25924848

414. Doblas A, Herrera M, Venegas J, et al. Successful diaphragmatic pacing for idiopathic alveolar hypoventilation. Intensive Care Med 1990; 16: 469-471. PMID: 2269719

415. Morélot-Panzini C, Gonzalez-Bermejo J, Straus C, et al. Reversal of pulmonary hypertension after diaphragm pacing in an adult patient with congenital central hypoventilation syndrome. Int $J$ Artif Organs 2013; 36: 434-438. PMID: 23653299

416. Tatsumi K, Sakuma T, Kuriyama T, et al. Pulmonary circulation disorder and sleep-disordered breathing: Pathophysiology and treatment. Kekkaku 2005; 80: 663-666. [in Japanese]

417. Sasaki Y, Yamagishi F, Suzuki K, et al. Survival and pulmonary hemodynamics in patients with sequelae of pulmonary tuberculosis who received home oxygen therapy. Nihon Kyobu Shikkan Gakkai Zasshi 1997; 35: 511-517. [in Japanese]

418. Klinger JR. Group III Pulmonary Hypertension: Pulmonary hypertension associated with lung disease: Epidemiology, pathophysiology, and treatments. Cardiol Clin 2016; 34: $413-$ 433. PMID: 27443138

419. Kuriyama T. Summary report: The 1997 report of the respiratory failure study group of the MHLW 1998: 1-11. [in Japanese]

420. Han MK, McLaughlin VV, Criner GJ, et al. Pulmonary diseases and the heart. Circulation 2007; 116: 2992-3005. PMID: 18086941

421. Kessler R, Chaouat A, Schinkewitch P, et al. The obesityhypoventilation syndrome revisited: A prospective study of 34 consecutive cases. Chest 2001; 120: 369-376. PMID: 11502631

422. Sugerman HJ, Baron PL, Fairman RP, et al. Hemodynamic dysfunction in obesity hypoventilation syndrome and the effects of treatment with surgically induced weight loss. Ann Surg 1988; 207: 604-613. PMID: 3377570

423. Fein DG, Zaidi AN, Sulica R. Pulmonary hypertension due to common respiratory conditions: Classification, evaluation and management strategies. J Clin Med 2016; 5: 75. PMID: 27571110

424. Held M, Walthelm J, Baron S, et al. Functional impact of pulmonary hypertension due to hypoventilation and changes under noninvasive ventilation. Eur Respir J 2014; 43: 156-165. PMID: 23722614

425. Piper AJ, Grunstein RR. Obesity hypoventilation syndrome: Mechanisms and management. Am J Respir Crit Care Med 2011; 183: 292-298. PMID: 21037018

426. Guidelines for Diagnosis and Treatment of Cardiovascular Diseases. Guidelines for diagnosis and treatment of sleep disordered breathing in cardiovascular disease (JCS 2010). http:// www.j-circ.or.jp/guideline/pdf/JCS2010,momomura.h.pdf [in Japanese]

427. Sajkov D, Wang T, Saunders NA, et al. Continuous positive airway pressure treatment improves pulmonary hemodynamics in patients with obstructive sleep apnea. Am J Respir Crit Care Med 2002; 165: 152-158. PMID: 11790646

428. Arias MA, García-Río F, Alonso-Fernández A, et al. Pulmonary hypertension in obstructive sleep apnoea: Effects of continuous positive airway pressure: A randomized, controlled cross-over study. Eur Heart J 2006; 27: 1106-1113. PMID: 16497687

429. Fletcher EC, Schaaf JW, Miller J, et al. Long-term cardiopulmonary sequelae in patients with sleep apnea and chronic lung disease. Am Rev Respir Dis 1987; 135: 525-533. PMID: 3826878

430. Tal A, Leiberman A, Margulis G, et al. Ventricular dysfunction in children with obstructive sleep apnea: Radionuclide assessment. Pediatr Pulmonol 1988; 4: 139-143. PMID: 2836784

431. Zohar Y, Talmi YP, Frenkel $\mathrm{H}$, et al. Cardiac function in obstructive sleep apnea patients following uvulopalatopharyngoplasty. Otolaryngol Head Neck Surg 1992; 107: 390-394. PMID: 1383911

432. Madani MM, Auger WR, Pretorius V, et al. Pulmonary endarterectomy: Recent changes in a single institution's experience of more than 2,700 patients. Ann Thorac Surg 2012; 94: 97-103; discussion 103. PMID: 22626752

433. Ghofrani HA, D'Armini AM, Grimminger F, et al. CHEST-1 Study Group. Riociguat for the treatment of chronic thrombo- 
embolic pulmonary hypertension. $N$ Engl J Med 2013; 369: 319-329. PMID: 23883377

434. Sugimura K, Fukumoto Y, Satoh K, et al. Percutaneous transluminal pulmonary angioplasty markedly improves pulmonary hemodynamics and long-term prognosis in patients with chronic thromboembolic pulmonary hypertension. Circ J 2012; 76: 485-488. PMID: 22185711

435. Mizoguchi H, Ogawa A, Munemasa M, et al. Refined balloon pulmonary angioplasty for inoperable patients with chronic thromboembolic pulmonary hypertension. Circ Cardiovasc Interv 2012; 5: 748-755. PMID: 23192917

436. Kataoka M, Inami $\mathrm{T}$, Hayashida $\mathrm{K}$, et al. Percutaneous transluminal pulmonary angioplasty for the treatment of chronic thromboembolic pulmonary hypertension. Circ Cardiovasc Interv 2012; 5: 756-762. PMID: 23132237

437. Blauwet LA, Edwards WD, Tazelaar HD, et al. Surgical pathology of pulmonary thromboendarterectomy: A study of 54 cases from 1990 to 2001. Hum Pathol 2003; 34: 1290-1298. PMID: 14691915

438. Bernard J, Yi ES. Pulmonary thromboendarterectomy: A clinicopathologic study of 200 consecutive pulmonary thromboendarterectomy cases in one institution. Hum Pathol 2007; 38: 871-877. PMID: 17350667

439. Hosokawa K, Ishibashi-Ueda H, Kishi T, et al. Histopathological multiple recanalized lesion is critical element of outcome after pulmonary thromboendarterectomy. Int Heart J 2011; 52: 377-381. PMID: 22188712

440. Nakajima N, Kawazoe K, Ando M, et al. Surgical treatment for chronic pulmonary embolism. The Japanese Journal of Chest Diseases 1986; 34: 524-531.

441. Daily PO, Johnston GG, Simmons CJ, et al. Surgical management of chronic pulmonary embolism: Surgical treatment and late results. J Thorac Cardiovasc Surg 1980; 79: 523-531. PMID: 7359931

442. Daily PO, Dembitsky WP, Iversen S, et al. Risk factors for pulmonary thromboendarterectomy. $J$ Thorac Cardiovasc Surg 1990; 99: 670-678. PMID: 2319789

443. Jamieson SW, Auger WR, Fedullo PF, et al. Experience and results with 150 pulmonary thromboendarterectomy operations over a 29-month period. J Thorac Cardiovasc Surg 1993; 106: 116-126; discussion 126-127. PMID: 8320990

444. Jamieson SW, Kapelanski DP, Sakakibara N, et al. Pulmonary endarterectomy: Experience and lessons learned in 1,500 cases. Ann Thorac Surg 2003; 76: 1457-1462; discussion 1462-1464. PMID: 14602267

445. Ando M, Takamoto S, Okita Y, et al. Operation for chronic pulmonary thromboembolism accompanied by thrombophilia in 8 patients. Ann Thorac Surg 1998; 66: 1919-1924. PMID: 9930469

446. Ando M, Okita Y, Tagusari O, et al. Surgical treatment for chronic thromboembolic pulmonary hypertension under profound hypothermia and circulatory arrest in 24 patients. $J$ Card Surg 1999; 14: 377-385. PMID: 10875595

447. Masuda M, Nakajima N. Our experience of surgical treatment for chronic pulmonary thromboembolism. Ann Thorac Cardiovasc Surg 2001; 7: 261-265. PMID: 11743851

448. Masuda M, Okumura M, Doki Y, et al. Committee for Scientific Affairs, The Japanese Association for Thoracic Surgery. Thoracic and cardiovascular surgery in Japan during 2014: Annual report by The Japanese Association for Thoracic Surgery. Gen Thorac Cardiovasc Surg 2016; 64: 665-697. PMID: 27590348

449. Thistlethwaite PA, Mo M, Madani MM, et al. Operative classification of thromboembolic disease determines outcome after pulmonary endarterectomy. J Thorac Cardiovasc Surg 2002; 124: $1203-1211$. PMID: 12447188

450. Ogino H, Ando M, Matsuda H, et al. Japanese single-center experience of surgery for chronic thromboembolic pulmonary hypertension. Ann Thorac Surg 2006; 82: 630-636. PMID: 16863777

451. Madani MM. Surgical treatment of chronic thromboembolic pulmonary hypertension: Pulmonary thromboendarterectomy. Methodist Debakey Cardiovasc J 2016; 12: 213-218. PMID: 28289496

452. Hartz RS, Byrne JG, Levitsky S, et al. Predictors of mortality in pulmonary thromboendarterectomy. Ann Thorac Surg 1996; 62: 1255-1259; discussion 1259-1260. PMID: 8893554

453. Thistlethwaite PA, Kemp A, Du L, et al. Outcomes of pulmonary endarterectomy for treatment of extreme thromboembolic pulmonary hypertension. J Thorac Cardiovasc Surg 2006; 131:
307-313. PMID: 16434258

454. Reesink HJ, Marcus JT, Tulevski II, et al. Reverse right ventricular remodeling after pulmonary endarterectomy in patients with chronic thromboembolic pulmonary hypertension: Utility of magnetic resonance imaging to demonstrate restoration of the right ventricle. J Thorac Cardiovasc Surg 2007; 133: 58-64. PMID: 17198781

455. Menzel T, Kramm T, Wagner S, et al. Improvement of tricuspid regurgitation after pulmonary thromboendarterectomy. Ann Thorac Surg 2002; 73: 756-761. PMID: 11899178

456. Sadeghi HM, Kimura BJ, Raisinghani A, et al. Does lowering pulmonary arterial pressure eliminate severe functional tricuspid regurgitation?: Insights from pulmonary thromboendarterectomy. J Am Coll Cardiol 2004; 44: 126-132. PMID: 15234420

457. Tscholl D, Langer F, Wendler O, et al. Pulmonary thromboendarterectomy--risk factors for early survival and hemodynamic improvement. Eur J Cardiothorac Surg 2001; 19: 771-776. PMID: 11404129

458. Mo M, Kapelanski DP, Mitruka SN, et al. Reoperative pulmonary thromboendarterectomy. Ann Thorac Surg 1999; 68: 1770-1776; discussion 1776-1777. PMID: 10585057

459. Madani MM, Wittine LM, Auger WR, et al. Chronic thromboembolic pulmonary hypertension in pediatric patients. $J$ Thorac Cardiovasc Surg 2011; 141: 624-630. PMID: 20800245

460. Thistlethwaite PA, Auger WR, Madani MM, et al. Pulmonary thromboendarterectomy combined with other cardiac operations: Indications, surgical approach, and outcome. Ann Thorac Surg 2001; 72: 13-17; discussion 17-19. PMID: 11465167

461. Nagaya N, Sasaki N, Ando M, et al. Prostacyclin therapy before pulmonary thromboendarterectomy in patients with chronic thromboembolic pulmonary hypertension. Chest 2003; 123: 338 -343. PMID: 12576349

462. Jensen KW, Kerr KM, Fedullo PF, et al. Pulmonary hypertensive medical therapy in chronic thromboembolic pulmonary hypertension before pulmonary thromboendarterectomy. Circulation 2009; 120: 1248-1254. PMID: 19752321

463. Mayer E, Jenkins D, Lindner J, et al. Surgical management and outcome of patients with chronic thromboembolic pulmonary hypertension: Results from an international prospective registry. J Thorac Cardiovasc Surg 2011; 141: 702-710. PMID: 21335128

464. Jenkins D, Mayer E, Screaton N, et al. State-of-the-art chronic thromboembolic pulmonary hypertension diagnosis and management. Eur Respir Rev 2012; 21: 32-39. PMID: 22379172

465. Kunihara T, Gerdts J, Groesdonk H, et al. Predictors of postoperative outcome after pulmonary endarterectomy from a 14-year experience with 279 patients. Eur J Cardiothorac Surg 2011; 40: 154-161. PMID: 21353588

466. Ishida $\mathrm{K}$, Masuda $\mathrm{M}$, Tanaka $\mathrm{H}$, et al. Mid-term results of surgery for chronic thromboembolic pulmonary hypertension. Interact Cardiovasc Thorac Surg 2009; 9: 626-629. PMID: 19608561

467. Freed DH, Thomson BM, Berman M, et al. Survival after pulmonary thromboendarterectomy: Effect of residual pulmonary hypertension. J Thorac Cardiovasc Surg 2011; 141: $383-$ 387. PMID: 20471039

468. Ishida K, Masuda M, Tanabe $\mathrm{N}$, et al. Long-term outcome after pulmonary endarterectomy for chronic thromboembolic pulmonary hypertension. J Thorac Cardiovasc Surg 2012; 144: 321-326. PMID: 21992851

469. Galiè N, Kim NH. Pulmonary microvascular disease in chronic thromboembolic pulmonary hypertension. Proc Am Thorac Soc 2006; 3: 571-576. PMID: 16963536

470. Simonneau G, D'Armini AM, Ghofrani HA, et al. Predictors of long-term outcomes in patients treated with riociguat for chronic thromboembolic pulmonary hypertension: Data from the CHEST-2 open-label, randomised, long-term extension trial. Lancet Respir Med 2016; 4: 372-380. PMID: 27067478

471. Bresser P, Fedullo PF, Auger WR, et al. Continuous intravenous epoprostenol for chronic thromboembolic pulmonary hypertension. Eur Respir J 2004; 23: 595-600. PMID: 15083760

472. Cabrol S, Souza R, Jais X, et al. Intravenous epoprostenol in inoperable chronic thromboembolic pulmonary hypertension. J Heart Lung Transplant 2007; 26: 357-362. PMID: 17403477

473. Ono F, Nagaya N, Okumura H, et al. Effect of orally active prostacyclin analogue on survival in patients with chronic thromboembolic pulmonary hypertension without major vessel obstruction. Chest 2003; 123: 1583-1588. PMID: 12740277

474. Olschewski H, Simonneau G, Galiè N, et al. Aerosolized Iloprost Randomized Study Group. Inhaled iloprost for severe pulmonary hypertension. N Engl J Med 2002; 347: 322-329. 
PMID: 12151469

475. Skoro-Sajer N, Bonderman D, Wiesbauer F, et al. Treprostinil for severe inoperable chronic thromboembolic pulmonary hypertension. J Thromb Haemost 2007; 5: 483-489. PMID: 17319903

476. Reichenberger F, Voswinckel R, Enke B, et al. Long-term treatment with sildenafil in chronic thromboembolic pulmonary hypertension. Eur Respir J 2007; 30: 922-927. PMID: 17690123

477. Jaï X, D'Armini AM, Jansa P, et al. Bosentan Effects in iNopErable Forms of chronIc Thromboembolic pulmonary hypertension Study Group. Bosentan for treatment of inoperable chronic thromboembolic pulmonary hypertension: BENEFiT (Bosentan Effects in iNopErable Forms of chronIc Thromboembolic pulmonary hypertension), a randomized, placebocontrolled trial. J Am Coll Cardiol 2008; 52: 2127-2134. PMID: 19095129

478. Reesink HJ, Surie S, Kloek JJ, et al. Bosentan as a bridge to pulmonary endarterectomy for chronic thromboembolic pulmonary hypertension. J Thorac Cardiovasc Surg 2010; 139: 85-91. PMID: 19660388

479. Riedel M, Stanek V, Widimsky J, et al. Longterm follow-up of patients with pulmonary thromboembolism: Late prognosis and evolution of hemodynamic and respiratory data. Chest 1982; 81: 151-158. PMID: 7056079

480. Feinstein JA, Goldhaber SZ, Lock JE, et al. Balloon pulmonary angioplasty for treatment of chronic thromboembolic pulmonary hypertension. Circulation 2001; 103: 10-13. PMID: 11136677

481. Statement for balloon pulmonary angioplasty for chronic thromboembolic pulmonary hypertension (JCS 2014). http:// www.j-circ.or.jp/guideline/pdf/JCS2014_ito_d.pdf [in Japanese]

482. The Japanese Circulation Society. Research on clinical practice for patients with cardiovascular disorders (conducted and published in 2016). http://www.j-circ.or.jp/jittai_chosa/jittai_ chosa2015web.pdf [in Japanese]

483. Thistlethwaite PA, Madani M, Jamieson SW. Outcomes of pulmonary endarterectomy surgery. Semin Thorac Cardiovasc Surg 2006; 18: 257-264. PMID: 17185189

484. Andreassen AK, Ragnarsson A, Gude E, et al. Balloon pulmonary angioplasty in patients with inoperable chronic thromboembolic pulmonary hypertension. Heart 2013; 99: 1415-1420. PMID: 23846611

485. Kurzyna M, Darocha S, Koteja A, et al. Balloon pulmonary angioplasty for chronic thromboembolic pulmonary hypertension. Postepy Kardiol Interwencyjnej 2015; 11: 1-4. PMID: 25848362

486. Kawakami T, Ogawa A, Miyaji K, et al. Novel angiographic classification of each vascular lesion in chronic thromboembolic pulmonary hypertension based on selective angiogram and results of balloon pulmonary angioplasty. Circ Cardiovasc Interv 2016; 9: e003318. PMID: 27729418

487. Inami T, Kataoka M, Shimura N, et al. Pressure-wire-guided percutaneous transluminal pulmonary angioplasty: A breakthrough in catheter-interventional therapy for chronic thromboembolic pulmonary hypertension. JACC Cardiovasc Interv 2014; 7: 1297-1306. PMID: 25459043

488. Bacha EA, Wright CD, Grillo HC, et al. Surgical treatment of primary pulmonary sarcomas. Eur J Cardiothorac Surg 1999; 15: 456-460. PMID: 10371121

489. Nakahira A, Ogino H, Sasaki H, et al. Long-term survival of a pulmonary artery sarcoma produced by aggressive surgical resection and adjuvant chemoradiotherapy. Eur J Cardiothorac Surg 2007; 32: 388-390. PMID: 17566754

490. Stella F, Davoli F, Brandolini J, et al. Pulmonary artery leiomyosarcoma successfully treated by right pneumonectomy. Asian Cardiovasc Thorac Ann 2009; 17: 513-515. PMID: 19917796

491. Tavora F, Miettinen M, Fanburg-Smith J, et al. Pulmonary artery sarcoma: A histologic and follow-up study with emphasis on a subset of low-grade myofibroblastic sarcomas with a good long-term follow-up. Am J Surg Pathol 2008; 32: 1751-1761. PMID: 18779732

492. Kreutzer J, Landzberg MJ, Preminger TJ, et al. Isolated peripheral pulmonary artery stenoses in the adult. Circulation 1996; 93: 1417-1423. PMID: 8641032

493. Yanagisawa R, Kataoka M, Inami T, et al. Intravascular imagingguided percutaneous transluminal pulmonary angioplasty for peripheral pulmonary stenosis and pulmonary Takayasu arteritis. J Heart Lung Transplant 2016; 35: 537-540. PMID: 26776920

494. Pulmonary pulseless disease: Pulmonary involvement in socalled Takayasu's disease. Clinical conference in cardiology from the third medical division, Kyoto University Hospital, Kyoto, Japan. Chest 1978; 73: 651-657. PMID: 25748

495. Erkan F, Gül A, Tasali E. Pulmonary manifestations of Behçet's disease. Thorax 2001; 56: 572-578. PMID: 11413359

496. Tunaci M, Ozkorkmaz B, Tunaci A, et al. CT findings of pulmonary artery aneurysms during treatment for Behçet's disease. AJR Am J Roentgenol 1999; 172: 729-733. PMID: 10063870

497. Saji T, Monma K, Shibata T, et al. Results of a nation-wide survey on primary pulmonary hypertension in children - Conditions of patients indicated for lung transplantation (First report). Pediatric Cardiology and Cardiac Surgery 2000; 16: 230-237. [in Japanese]

498. Moledina S, Hislop AA, Foster H, et al. Childhood idiopathic pulmonary arterial hypertension: A national cohort study. Heart 2010; 96: 1401-1406. PMID: 20406768

499. van Loon RL, Roofthooft MT, Hillege HL, et al. Pediatric pulmonary hypertension in the Netherlands: Epidemiology and characterization during the period 1991 to 2005. Circulation 2011; 124: 1755-1764. PMID: 21947294

500. Berger RM, Beghetti M, Humpl T, et al. Clinical features of paediatric pulmonary hypertension: A registry study. Lancet 2012; 379: 537-546. PMID: 22240409

501. Goncharova EA, Gladwin MT, Kawut SM. Update in pulmonary vascular diseases 2014. Am J Respir Crit Care Med 2015; 192: 544-550. PMID: 26561677

502. Sandoval J, Bauerle O, Gomez A, et al. Primary pulmonary hypertension in children: Clinical characterization and survival. $J$ Am Coll Cardiol 1995; 25: 466-474. PMID: 7829802

503. Hill KD, Lim DS, Everett AD, et al. Assessment of pulmonary hypertension in the pediatric catheterization laboratory: Current insights from the magic registry. Catheter Cardiovasc Interv 2010; 76: 865-873. PMID: 20549685

504. Beghetti M, Schulze-Neick I, Berger RM, et al. TOPP Investigators. Haemodynamic characterisation and heart catheterisation complications in children with pulmonary hypertension: Insights from the Global TOPP Registry (tracking outcomes and practice in paediatric pulmonary hypertension). Int J Cardiol 2016; 203: 325-330. PMID: 26583838

505. Matsuura H. Cardiac catheterization in children with pulmonary arterial hypertension. Pediatr Int 2017; 59: 3-9. PMID: 27599454

506. Jone PN, Ivy DD. Echocardiography in pediatric pulmonary hypertension. Front Pediatr 2014; 2: 124. PMID: 25429362

507. Barst RJ, McGoon MD, Elliott CG, et al. Survival in childhood pulmonary arterial hypertension: Insights from the registry to evaluate early and long-term pulmonary arterial hypertension disease management. Circulation 2012; 125: 113-122. PMID: 22086881

508. Abman SH, Hansmann G, Archer SL, et al. American Heart Association Council on Cardiopulmonary, Critical Care, Perioperative and Resuscitation; Council on Clinical Cardiology; Council on Cardiovascular Disease in the Young; Council on Cardiovascular Radiology and Intervention; Council on Cardiovascular Surgery and Anesthesia; and the American Thoracic Society. Pediatric Pulmonary Hypertension: Guidelines From the American Heart Association and American Thoracic Society. Circulation 2015; 132: 2037-2099. PMID: 26534956

509. Hansmann G, Apitz C. Treatment of children with pulmonary hypertension: Expert consensus statement on the diagnosis and treatment of paediatric pulmonary hypertension: The European Paediatric Pulmonary Vascular Disease Network, endorsed by ISHLT and DGPK. Heart 2016; 102: ii67-ii85. PMID: 27053700

510. Douwes JM, Hegeman AK, van der Krieke MB, et al. Sixminute walking distance and decrease in oxygen saturation during the six-minute walk test in pediatric pulmonary arterial hypertension. Int J Cardiol 2016; 202: 34-39. PMID: 26386916

511. Chida A, Shintani M, Yagi H, et al. Outcomes of childhood pulmonary arterial hypertension in BMPR 2 and ALK1 mutation carriers. Am J Cardiol 2012; 110: 586-593. PMID: 22632830

512. Takatsuki S, Nakayama T, Jone PN, et al. Tissue Doppler imaging predicts adverse outcome in children with idiopathic pulmonary arterial hypertension. J Pediatr 2012; 161: $1126-$ 1131. PMID: 22748515

513. Saji T, Nakayama T, Takatsuki S, et al. The real world of medical treatment of pulmonary arterial hypertension: Small evidence, but heavy cornerstone. Pediatric Cardiology and Cardiac Surgery 2015; 31: 157-183. [in Japanese]

514. Abman SH, Kinsella JP, Rosenzweig EB, et al. Pediatric Pulmonary Hypertension Network (PPHNet): Implications of 
the U.S. Food and Drug Administration warning against the use of sildenafil for the treatment of pediatric pulmonary hypertension. Am J Respir Crit Care Med 2013; 187: 572-575. PMID: 23220921

515. Baruteau AE, Serraf A, Lévy M, et al. Potts shunt in children with idiopathic pulmonary arterial hypertension: Long-term results. Ann Thorac Surg 2012; 94: 817-824. PMID: 22704329

516. Satoh M, Aso K, Nakayama T, et al. Autoimmune thyroid disease in children and adolescents with idiopathic pulmonary arterial hypertension. Circ J 2010; 74: 371 -374. PMID: 20009390

517. Trapp CM, Elder RW, Gerken AT, et al. Pediatric pulmonary arterial hypertension and hyperthyroidism: A potentially fatal combination. J Clin Endocrinol Metab 2012; 97: 2217-2222. PMID: 22622024

518. Roofthooft MT, Douwes JM, Vrijlandt EJ, et al. Frequency and prognostic significance of hemoptysis in pediatric pulmonary arterial hypertension. Am J Cardiol 2013; 112: 1505-1509. PMID: 23972345

519. Haworth SG, Hislop AA. Treatment and survival in children with pulmonary arterial hypertension: The UK pulmonary hypertension service for children 2001-2006. Heart 2009; 95: 312-317. PMID: 18952635

520. Fraisse A, Jais X, Schleich JM, et al. Characteristics and prospective 2-year follow-up of children with pulmonary arterial hypertension in France. Arch Cardiovasc Dis 2010; 103: 66-74. PMID: 20226425

521. Barst RJ, Ertel SI, Beghetti M, et al. Pulmonary arterial hypertension: A comparison between children and adults. Eur Respir $J$ 2011; 37: 665-677. PMID: 21357924

522. Yamaki S, Horiuchi T, Takahashi T. Pulmonary changes in congenital heart disease with Down's syndrome: Their significance as a cause of postoperative respiratory failure. Thorax 1985; 40: 380-386. PMID: 3161204

523. Wood P. The Eisenmenger syndrome or pulmonary hypertension with reversed central shunt: I. Br Med J 1958; 2: 701-709. PMID: 13572874

524. Ivy DD, Abman SH, Barst RJ, et al. Pediatric pulmonary hypertension. J Am Coll Cardiol 2013; 62: D117-D126. PMID: 24355636

525. Naeije R, Vachiery JL, Yerly P, et al. The transpulmonary pressure gradient for the diagnosis of pulmonary vascular disease. Eur Respir J 2013; 41: 217-223. PMID: 22936712

526. Chowdhury UK, Govindappa RM, Das P, et al. Histomorphometric analysis of intrapulmonary vessels in patients undergoing bidirectional Glenn shunt and total cavopulmonary connection. J Thorac Cardiovasc Surg 2010; 140: 1251-1256.e1-e14. PMID: 20621309

527. Ishida $\mathrm{H}$, Kogaki $\mathrm{S}$, Ichimori $\mathrm{H}$, et al. Overexpression of endothelin-1 and endothelin receptors in the pulmonary arteries of failed Fontan patients. Int J Cardiol 2012; 159: 34-39. PMID: 21356562

528. Hebert A, Mikkelsen UR, Thilen U, et al. Bosentan improves exercise capacity in adolescents and adults after Fontan operation: The TEMPO (Treatment With Endothelin Receptor Antagonist in Fontan Patients, a Randomized, PlaceboControlled, Double-Blind Study Measuring Peak Oxygen Consumption) study. Circulation 2014; 130: 2021-2030. PMID: 25446057

529. Derk G, Houser L, Miner P, et al. Efficacy of endothelin blockade in adults with Fontan physiology. Congenit Heart Dis 2015; 10: E11-E16. PMID: 24890846

530. Cerro MJ, Abman S, Diaz G, et al. A consensus approach to the classification of pediatric pulmonary hypertensive vascular disease: Report from the PVRI Pediatric Taskforce, Panama 2011. Pulm Circ 2011; 1: 286-298. PMID: 21874158

531. Ivy D. Pulmonary Hypertension in Children. Cardiol Clin 2016; 34: 451-472. PMID: 27443141

532. Latus H, Kuehne T, Beerbaum P, et al. Cardiac MR and CT imaging in children with suspected or confirmed pulmonary hypertension/pulmonary hypertensive vascular disease. Expert consensus statement on the diagnosis and treatment of paediatric pulmonary hypertension. The European Paediatric Pulmonary Vascular Disease Network, endorsed by ISHLT and DGPK. Heart 2016; 102: ii30-ii35. PMID: 27053695

533. McLaughlin VV, Archer SL, Badesch DB, et al. American College of Cardiology Foundation Task Force on Expert Consensus Documents. ACCF/AHA 2009 expert consensus document on pulmonary hypertension a report of the American College of Cardiology Foundation Task Force on Expert Consensus Documents and the American Heart Association developed in collaboration with the American College of Chest Physicians; American Thoracic Society, Inc.; and the Pulmonary Hypertension Association. J Am Coll Cardiol 2009; 53: $1573-$ 1619. PMID: 19389575

534. Lopes AA, Barst RJ, Haworth SG, et al. Repair of congenital heart disease with associated pulmonary hypertension in children: What are the minimal investigative procedures?: Consensus statement from the Congenital Heart Disease and Pediatric Task Forces, Pulmonary Vascular Research Institute (PVRI). Pulm Circ 2014; 4: 330-341. PMID: 25006452

535. Kozlik-Feldmann R, Hansmann G, Bonnet D, et al. Pulmonary hypertension in children with congenital heart disease (PAHCHD, PPHVD-CHD). Expert consensus statement on the diagnosis and treatment of paediatric pulmonary hypertension. The European Paediatric Pulmonary Vascular Disease Network, endorsed by ISHLT and DGPK. Heart 2016; 102: ii42-ii48. PMID: 27053697

536. Pattathu J, Gorenflo M, Hilgendorff A, et al. Genetic testing and blood biomarkers in paediatric pulmonary hypertension. Expert consensus statement on the diagnosis and treatment of paediatric pulmonary hypertension. The European Paediatric Pulmonary Vascular Disease Network, endorsed by ISHLT and DGPK. Heart 2016; 102: ii36-ii41. PMID: 27053696

537. Barst RJ. Pharmacologically induced pulmonary vasodilatation in children and young adults with primary pulmonary hypertension. Chest 1986; 89: 497-503. PMID: 2869919

538. Sitbon O, Humbert M, Ioos V, et al. Who benefits from longterm calcium-channel blocker therapy in primary pulmonary hypertension? (abstr). Am J Respir Crit Care Med 2003; 167: A440.

539. Zijlstra WM, Douwes JM, Rosenzweig EB, et al. Survival differences in pediatric pulmonary arterial hypertension: Clues to a better understanding of outcome and optimal treatment strategies. J Am Coll Cardiol 2014; 63: 2159-2169. PMID: 24681143

540. Rosenkranz S, Ghofrani HA, Beghetti M, et al. Riociguat for pulmonary arterial hypertension associated with congenital heart disease. Heart 2015; 101: 1792-1799. PMID: 26135803

541. Apostolopoulou SC, Papagiannis J, Rammos S. Bosentan induces clinical, exercise and hemodynamic improvement in a pre-transplant patient with plastic bronchitis after Fontan operation. J Heart Lung Transplant 2005; 24: 1174-1176. PMID: 16102469

542. Goldberg DJ, French B, McBride MG, et al. Impact of oral sildenafil on exercise performance in children and young adults after the fontan operation: A randomized, double-blind, placebocontrolled, crossover trial. Circulation 2011; 123: 1185-1193. PMID: 21382896

543. Giardini A, Balducci A, Specchia S, et al. Effect of sildenafil on haemodynamic response to exercise and exercise capacity in Fontan patients. Eur Heart $J$ 2008; 29: 1681-1687. PMID: 18534975

544. Ovaert C, Thijs D, Dewolf D, et al. The effect of bosentan in patients with a failing Fontan circulation. Cardiol Young 2009; 19: 331 -339. PMID: 19519964

545. Hirono K, Yoshimura N, Taguchi M, et al. Bosentan induces clinical and hemodynamic improvement in candidates for rightsided heart bypass surgery. $J$ Thorac Cardiovasc Surg 2010; 140: 346-351. PMID: 20434177

546. Beghetti M. Fontan and the pulmonary circulation: A potential role for new pulmonary hypertension therapies. Heart 2010; 96: 911-916. PMID: 20538665

547. Hebert A, Jensen AS, Idorn L, et al. The effect of bosentan on exercise capacity in Fontan patients; rationale and design for the TEMPO study. BMC Cardiovasc Disord 2013; 13: 36. PMID: 23663658

548. Walsh-Sukys MC, Tyson JE, Wright LL, et al. Persistent pulmonary hypertension of the newborn in the era before nitric oxide: Practice variation and outcomes. Pediatrics 2000; 105: 14-20. PMID: 10617698

549. Aikio O, Metsola J, Vuolteenaho R, et al. Transient defect in nitric oxide generation after rupture of fetal membranes and responsiveness to inhaled nitric oxide in very preterm infants with hypoxic respiratory failure. J Pediatr 2012; 161: $397-$ 403.e1. PMID: 22554621

550. Ramachandrappa A, Rosenberg ES, Wagoner S, et al. Morbidity and mortality in late preterm infants with severe hypoxic respiratory failure on extra-corporeal membrane oxygenation. $J$ Pediatr 2011; 159: 192 -198.e3. PMID: 21459387

551. Konduri GG, Vohr B, Robertson C, et al. Neonatal Inhaled 
Nitric Oxide Study Group. Early inhaled nitric oxide therapy for term and near-term newborn infants with hypoxic respiratory failure: Neurodevelopmental follow-up. J Pediatr 2007; 150: 235-240.e1. PMID: 17307536

552. Hernández-Díaz S, Van Marter LJ, Werler MM, et al. Risk factors for persistent pulmonary hypertension of the newborn. Pediatrics 2007; 120: e272-e282. PMID: 17671038

553. Van Marter LJ, Hernandez-Diaz S, Werler MM, et al. Nonsteroidal antiinflammatory drugs in late pregnancy and persistent pulmonary hypertension of the newborn. Pediatrics 2013; 131: 79-87. PMID: 23209104

554. Grigoriadis S, Vonderporten EH, Mamisashvili L, et al. Prenatal exposure to antidepressants and persistent pulmonary hypertension of the newborn: Systematic review and meta-analysis. BMJ 2014; 348: f6932. PMID: 24429387

555. Stankiewicz P, Sen P, Bhatt SS, et al. Genomic and genic deletions of the FOX gene cluster on 16q24.1 and inactivating mutations of FOXF1 cause alveolar capillary dysplasia and other malformations. Am J Hum Genet 2009; 84: 780-791. PMID: 19500772

556. Shah N, Natarajan G, Aggarwal S. B-type natriuretic peptide: Biomarker of persistent pulmonary hypertension of the newborn? Am J Perinatol 2015; 32: 1045-1049. PMID: 26368790

557. Hilgendorff A, Apitz C, Bonnet D, et al. Pulmonary hypertension associated with acute or chronic lung diseases in the preterm and term neonate and infant: The European Paediatric Pulmonary Vascular Disease Network, endorsed by ISHLT and DGPK. Heart 2016; 102: ii49-ii56. PMID: 27053698

558. Castillo A, Sola A, Baquero H, et al. Pulse oxygen saturation levels and arterial oxygen tension values in newborns receiving oxygen therapy in the neonatal intensive care unit: Is $85 \%$ to $93 \%$ an acceptable range? Pediatrics 2008; 121: 882-889. PMID: 18450890

559. El Shahed AI, Dargaville PA, Ohlsson A, et al. Surfactant for meconium aspiration syndrome in term and late preterm infants. Cochrane Database Syst Rev 2014; (12): CD002054. PMID: 25504256

560. Finer NN, Barrington KJ. Nitric oxide for respiratory failure in infants born at or near term. Cochrane Database Syst Rev 2006; (4): CD000399. PMID: 17054129

561. Askie LM, Ballard RA, Cutter GR, et al. Meta-analysis of Preterm Patients on Inhaled Nitric Oxide Collaboration. Inhaled nitric oxide in preterm infants: An individual-patient data meta-analysis of randomized trials. Pediatrics 2011; 128: 729-739. PMID: 21930540

562. Kinsella JP. Inhaled nitric oxide in the term newborn. Early Hum Dev 2008; 84: 709-716. PMID: 18930613

563. Clark RH, Kueser TJ, Walker MW, et al. Clinical Inhaled Nitric Oxide Research Group. Low-dose nitric oxide therapy for persistent pulmonary hypertension of the newborn. $N$ Engl $J$ Med 2000; 342: 469-474. PMID: 10675427

564. Extracorporeal Life Support Organization (ELSO). Guidelines for Neonatal Respiratory Failure. https://www.elso.org/ Portals/0/IGD/Archive/FileManager/8588d1a580cusersshyerd ocumentselsoguidelinesforneonatalrespiratoryfailure13.pdf

565. Baquero H, Soliz A, Neira F, et al. Oral sildenafil in infants with persistent pulmonary hypertension of the newborn: A pilot randomized blinded study. Pediatrics 2006; 117: 1077-1083. PMID: 16585301

566. Kelly LE, Ohlsson A, Shah PS. Sildenafil for pulmonary hypertension in neonates. Cochrane Database Syst Rev 2017; 8: CD005494. PMID: 28777888

567. Kelly LK, Porta NF, Goodman DM, et al. Inhaled prostacyclin for term infants with persistent pulmonary hypertension refractory to inhaled nitric oxide. J Pediatr 2002; 141: 830-832. PMID: 12461501

568. Rosenberg AA, Kennaugh J, Koppenhafer SL, et al. Elevated immunoreactive endothelin-1 levels in newborn infants with persistent pulmonary hypertension. J Pediatr 1993; 123: $109-$ 114. PMID: 8320603

569. Mohamed WA, Ismail M. A randomized, double-blind, placebocontrolled, prospective study of bosentan for the treatment of persistent pulmonary hypertension of the newborn. J Perinatol 2012; 32: 608-613. PMID: 22076415

570. Steinhorn RH, Fineman J, Kusic-Pajic A, et al. FUTURE-4 study investigators. Bosentan as Adjunctive Therapy for Persistent Pulmonary Hypertension of the Newborn: Results of the Randomized Multicenter Placebo-Controlled Exploratory Trial. $J$ Pediatr 2016; 177: 90-96.e3. PMID: 27502103

571. The Ministry of Health, Labour and Welfare. Intractable diseases designated as of January 1, 2015 (new and revised diseases): Pulmonary arterial hypertension (Notice No. 86), Descriptions and diagnostic criteria. http://www.mhlw.go.jp/ file/06-Seisakujouhou-10900000-Kenkoukyoku/0000157754. docx (Accessed in December 2017). [in Japanese]

572. The Ministry of Health, Labour and Welfare. Intractable diseases designated as of January 1, 2015 (new and revised diseases): Chronic thromboembolic pulmonary hypertension (Notice No. 88), Descriptions and diagnostic criteria. http://www. mhlw.go.jp/file/06-Seisakujouhou-10900000-Kenkoukyoku/ 0000157770.docx (Accessed in December 2017). [in Japanese]

573. The Ministry of Health, Labour and Welfare. Intractable diseases designated as of January 1, 2015 (new and revised diseases): Pulmonary vein occlusion/pulmonary capillary haemangiomatosis (Notice No. 87), Descriptions and diagnostic criteria. http://www.mhlw.go.jp/file/06-Seisakujouhou-109000 00-Kenkoukyoku/0000157867.docx (Accessed in December 2017). [in Japanese]

\section{Appendix 1}

Group Leader:

- Keiichi Fukuda, Department of Cardiology, Keio University School of Medicine

Members:

- Hiroshi Date, Department of Thoracic Surgery, Graduate School of Medicine, Kyoto University

- Shozaburo Doi, Department of Pediatrics, Perinatal and Maternal Medicine, Graduate School, Tokyo Medical and Dental University

- Yoshihiro Fukumoto, Division of Cardiovascular Medicine, Department of Internal Medicine, Kurume University School of Medicine

- Norihide Fukushima, Department of Transplant Medicine, National Cerebral and Cardiovascular Center

- Masaru Hatano, Department of Cardiovascular Medicine/Therapeutic Strategy for Heart Failure, The University of Tokyo Hospital

- Hiroshi Ito, Department of Cardiovascular Medicine, Field of Functional Physiology, Okayama University Graduate School of Medicine

- Masataka Kuwana, Department of Allergy and Rheumatology, Nippon Medical School

- Hiromi Matsubara, Department of Clinical Science, National Hospital Organization Okayama Medical Center

- Shin-ichi Momomura, Cardiovascular Medicine, Saitama Medical Center, Jichi Medical University

- Masaharu Nishimura, Department of Respiratory Medicine,
Hokkaido University Graduate School of Medicine

- Hitoshi Ogino, Department of Cardiovascular Surgery, Tokyo Medical University

- Toru Satoh, Internal Medicine II, Kyorin University School of Medicine

- Hiroaki Shimokawa, Department of Cardiovascular Medicine, Tohoku University Graduate School of Medicine

- Keiko Yamauchi-Takihara, Health and Counseling Center and Department of Cardiovascular Medicine, Osaka University Graduate School of Medicine

- Koichiro Tatsumi, Department of Respirology, Graduate School of Medicine, Chiba University

- Hatsue Ishibashi-Ueda, Department of Pathology, National Cerebral and Cardiovascular Center

- Norikazu Yamada, Cardiovascular Center, Kuwana City Medical Center

- Shunji Yoshida, Department of Rheumatology and Infectious Diseases, Fujita Health University Hospital

\section{Collaborators:}

- Kohtaro Abe, Department of Cardiovascular Medicine, Kyushu University Hospital

- Aiko Ogawa, Department of Clinical Science, National Hospital Organization Okayama Medical Center

- Takeshi Ogo, Division of Pulmonary Circulation, Department of Cardiovascular Medicine/Department of Advanced Medicine for 
Pulmonary Hypertension, National Cerebral and Cardiovascular Center

- Takatoshi Kasai, Department of Cardiovascular Medicine, Cardiovascular Respiratory Sleep Medicine, Juntendo University Graduate School of Medicine

- Masaharu Kataoka, Department of Cardiology, Keio University School of Medicine

- Takashi Kawakami, Department of Cardiology, Keio University School of Medicine

- Shigetoyo Kogaki, Department of Pediatrics and Neonatology, Osaka General Medical Center

- Mashio Nakamura, Nakamura Medical Clinic

- Tomotaka Nakayama, Department of Pediatrics, Toho University Medical Center Omori Hospital

- Mari Nishizaki, Department of Rehabilitation, National Hospital Organization, Okayama Medical Center

- Koichiro Sugimura, Department of Cardiovascular Medicine, Tohoku University Graduate School of Medicine

- Nobuhiro Tanabe, Department of Advanced Medicine in Pulmonary Hypertension, Graduate School of Medicine, Chiba University
- Ichizo Tsujino, Department of Respiratory Medicine, Faculty of Medicine and Graduate School of Medicine, Hokkaido University

- Atsushi Yao, Division for Health Service Promotion, The University of Tokyo

External Evaluation Committee Members:

- Takashi Akasaka, Department of Cardiovascular Medicine, Wakayama Medical University

- Motomi Ando, Daiyukai General Hospital Cardiovascular Center

- Takeshi Kimura, Department Cardiovascular Medicine, Graduate School of Medicine and Faculty of Medicine, Kyoto University

- Takayuki Kuriyama, Health Care Clinic Kuriyama

- Norifumi Nakanishi, Department of Cardiovascular Medicine, Minami Osaka Hospital

- Toshio Nakanishi, Department of Pediatric Cardiology, Tokyo Women's Medical University

- Hiroyuki Tsutsui, Department of Cardiovascular Medicine, Kyushu University Graduate School of Medical Sciences

(The affiliations of the members are as of March 2018)

Appendix 2 Disclosure of Potential Conflicts of Interest (COI): Guidelines for Treatment of Pulmonary Hypertension (JCS 2017/JPCPHS 2017)

\begin{tabular}{|c|c|c|c|c|c|c|c|c|c|}
\hline Author & $\begin{array}{l}\text { Employer/ } \\
\text { leadership } \\
\text { position } \\
\text { (private } \\
\text { company) }\end{array}$ & $\begin{array}{l}\text { Stake- } \\
\text { holder }\end{array}$ & $\begin{array}{l}\text { Patent } \\
\text { royalty }\end{array}$ & Honorarium & $\begin{array}{l}\text { Payment } \\
\text { for } \\
\text { manu- } \\
\text { scripts }\end{array}$ & Research grant & $\begin{array}{c}\text { Scholarship } \\
\text { (educational) } \\
\text { grant/endowed } \\
\text { chair }\end{array}$ & $\begin{array}{c}\text { Other } \\
\text { rewards }\end{array}$ & $\begin{array}{c}\text { Potential } \\
\text { COI of the } \\
\text { marital } \\
\text { partner, } \\
\text { first-degree } \\
\text { family } \\
\text { members, } \\
\text { or those } \\
\text { who share } \\
\text { income and } \\
\text { property }\end{array}$ \\
\hline $\begin{array}{l}\text { Group } \\
\text { leader: } \\
\text { Keiichi } \\
\text { Fukuda }\end{array}$ & $\begin{array}{l}\text { I'rom Co.,Ltd. } \\
\text { Heartseed Inc. }\end{array}$ & & & $\begin{array}{l}\text { Bayer Yakuhin, } \\
\text { Ltd. } \\
\text { Mitsubishi } \\
\text { Tanabe Pharma } \\
\text { Corporation }\end{array}$ & & $\begin{array}{l}\text { Sysmex } \\
\text { Corporation } \\
\text { Daiichi Sankyo } \\
\text { Company, } \\
\text { Limited. } \\
\text { Heartseed } \\
\text { Nipro } \\
\text { Corporation } \\
\text { AnGes, Inc. } \\
\text { Ajinomoto Co., } \\
\text { Inc. } \\
\text { Clinical trial: } \\
\text { Bayer } \\
\text { Yakuhin, Ltd. } \\
\text { Commissioned } \\
\text { study: Bayer } \\
\text { Yakuhin, Ltd. }\end{array}$ & $\begin{array}{l}\text { Bristol-Myers Squibb } \\
\text { Otsuka Pharmaceutical } \\
\text { Co., Ltd. } \\
\text { Orbusneich Medical K.K. } \\
\text { Mitsubishi Tanabe } \\
\text { Pharma Corporation } \\
\text { Pfizer Japan Inc. } \\
\text { Astellas Pharma Inc. } \\
\text { Takeda Pharmaceutical } \\
\text { Company Limited. } \\
\text { Kowa Pharmaceutical } \\
\text { Co., Ltd. } \\
\text { Nipro Corporation } \\
\text { Shinnihonseiyaku Co., } \\
\text { Ltd. }\end{array}$ & & \\
\hline
\end{tabular}




\begin{tabular}{|c|c|c|c|c|c|c|c|c|c|}
\hline Author & $\begin{array}{c}\text { Employer/ } \\
\text { leadership } \\
\text { position } \\
\text { (private } \\
\text { company) }\end{array}$ & $\begin{array}{l}\text { Stake- } \\
\text { holder }\end{array}$ & $\begin{array}{l}\text { Patent } \\
\text { royalty }\end{array}$ & Honorarium & $\begin{array}{l}\text { Payment } \\
\text { for } \\
\text { manu- } \\
\text { scripts }\end{array}$ & Research grant & $\begin{array}{c}\text { Scholarship } \\
\text { (educational) } \\
\text { grant/endowed } \\
\text { chair }\end{array}$ & $\begin{array}{l}\text { Other } \\
\text { rewards }\end{array}$ & $\begin{array}{c}\text { Potential } \\
\text { COI of the } \\
\text { marital } \\
\text { partner, } \\
\text { first-degree } \\
\text { family } \\
\text { members, } \\
\text { or those } \\
\text { who share } \\
\text { income and } \\
\text { property }\end{array}$ \\
\hline $\begin{array}{l}\text { Member: } \\
\text { Hiroshi Ito }\end{array}$ & & & & $\begin{array}{l}\text { Daiichi Sankyo } \\
\text { Company, } \\
\text { Limited. } \\
\text { Mitsubishi } \\
\text { Tanabe Pharma } \\
\text { Corporation } \\
\text { MSD K.K. } \\
\text { Bayer Yakuhin, } \\
\text { Ltd. } \\
\text { Boehringer } \\
\text { Ingelheim Corp. } \\
\text { Kowa } \\
\text { Pharmaceutical } \\
\text { Co., Ltd. }\end{array}$ & & \begin{tabular}{|l} 
Novartis \\
Pharma K.K. \\
Kowa \\
Pharmaceutical \\
Co., Ltd. \\
\\
\end{tabular} & $\begin{array}{l}\text { Daiichi Sankyo Company, } \\
\text { Limited. } \\
\text { Takeda Pharmaceutical } \\
\text { Company Limited. } \\
\text { Mitsubishi Tanabe } \\
\text { Pharma Corporation } \\
\text { MSD K.K. } \\
\text { Bayer Yakuhin, Ltd. } \\
\text { Boehringer Ingelheim } \\
\text { Corp. } \\
\text { Kowa Pharmaceutical } \\
\text { Co., Ltd. } \\
\text { Otsuka Pharmaceutical } \\
\text { Co., Ltd. } \\
\text { Sanofi K.K. } \\
\text { Mochida } \\
\text { Pharmaceutical } \\
\text { Co., Ltd. } \\
\text { Actelion Pharmaceuticals } \\
\text { Japan Ltd. } \\
\text { Boston Scientific } \\
\text { Corporation } \\
\text { BIOTRONIK Japan, Inc. } \\
\text { Fukuda Denshi Co., Ltd. } \\
\text { St. Jude Medical Japan } \\
\text { Co., Ltd. } \\
\text { Medtronic }\end{array}$ & & \\
\hline $\begin{array}{l}\text { Member: } \\
\text { Hitoshi } \\
\text { Ogino }\end{array}$ & $\begin{array}{l}\text { TERUMO } \\
\text { CORPORATION } \\
\text { JCT Co., Ltd. }\end{array}$ & & & & & & & & \\
\hline $\begin{array}{l}\text { Member: } \\
\text { Masataka } \\
\text { Kuwana }\end{array}$ & & & $\begin{array}{l}\text { Medical \& } \\
\text { Biological } \\
\text { Laboratories } \\
\text { Co., Ltd }\end{array}$ & $\begin{array}{l}\text { Actelion } \\
\text { Pharmaceuticals } \\
\text { Japan Ltd. } \\
\text { Pfizer Japan Inc. } \\
\text { Mitsubishi } \\
\text { Tanabe Pharma } \\
\text { Corporation } \\
\text { Ono } \\
\text { Pharmaceutical } \\
\text { Co., Ltd. } \\
\text { Nippon Shinyaku } \\
\text { Co., Ltd. } \\
\text { Eisai Co., Ltd. } \\
\text { Chugai } \\
\text { Pharmaceutical } \\
\text { Co.,Ltd. } \\
\text { Astellas Pharma } \\
\text { In. }\end{array}$ & & & $\begin{array}{l}\text { Actelion } \\
\text { Pharmaceuticals Japan } \\
\text { Ltd. } \\
\text { Pfizer Japan Inc. } \\
\text { Mitsubishi Tanabe } \\
\text { Pharma Corporation }\end{array}$ & & \\
\hline $\begin{array}{l}\text { Member: } \\
\text { Toru } \\
\text { Satoh }\end{array}$ & & & & $\begin{array}{l}\text { Actelion } \\
\text { Pharmaceuticals } \\
\text { Japan Ltd. }\end{array}$ & & \begin{tabular}{|l|} 
Actelion \\
Pharmaceuticals \\
Japan Ltd.
\end{tabular} & & & \\
\hline
\end{tabular}




\begin{tabular}{|c|c|c|c|c|c|c|c|c|c|}
\hline Author & $\begin{array}{l}\text { Employer/ } \\
\text { leadership } \\
\text { position } \\
\text { (private } \\
\text { company) }\end{array}$ & $\begin{array}{l}\text { Stake- } \\
\text { holder }\end{array}$ & $\begin{array}{l}\text { Patent } \\
\text { royalty }\end{array}$ & Honorarium & $\begin{array}{l}\text { Payment } \\
\text { for } \\
\text { manu- } \\
\text { scripts }\end{array}$ & Research grant & $\begin{array}{c}\text { Scholarship } \\
\text { (educational) } \\
\text { grant/endowed } \\
\text { chair }\end{array}$ & $\begin{array}{c}\text { Other } \\
\text { rewards }\end{array}$ & $\begin{array}{c}\text { Potential } \\
\text { COI of the } \\
\text { marital } \\
\text { partner, } \\
\text { first-degree } \\
\text { family } \\
\text { members, } \\
\text { or those } \\
\text { who share } \\
\text { income and } \\
\text { property }\end{array}$ \\
\hline $\begin{array}{l}\text { Member: } \\
\text { Hiroaki } \\
\text { Shimokawa }\end{array}$ & & & & $\begin{array}{l}\text { Bayer Yakuhin, } \\
\text { Ltd. } \\
\text { Boehringer } \\
\text { Ingelheim Corp. } \\
\text { Daiichi Sankyo } \\
\text { Company, } \\
\text { Limited. }\end{array}$ & & $\begin{array}{l}\text { Bayer Yakuhin, } \\
\text { Ltd. }\end{array}$ & $\begin{array}{l}\text { MSD K.K. (grant) } \\
\text { Nippon Shinyaku Co., } \\
\text { Ltd. } \\
\text { Daiichi Sankyo Company, } \\
\text { Limited. } \\
\text { Mitsubishi Tanabe } \\
\text { Pharma Corporation } \\
\text { AstraZeneca K.K. } \\
\text { Kyowa Hakko Kirin } \\
\text { Co., Ltd. } \\
\text { Boehringer Ingelheim } \\
\text { Corp. } \\
\text { Sumitomo Dainippon } \\
\text { Pharma Co., Ltd. } \\
\text { Novartis Pharma K.K. } \\
\text { Bayer Yakuhin, Ltd. } \\
\text { Daiichi Sankyo Company, } \\
\text { Limited. } \\
\text { Astellas Pharma Inc. } \\
\text { MSD K.K. (endowed } \\
\text { chair) } \\
\text { Otsuka Pharmaceutical } \\
\text { Co., Ltd. } \\
\text { GlaxoSmithKline K.K. } \\
\text { Kowa Pharmaceutical } \\
\text { Co., Ltd. } \\
\text { Shionogi \& Co., Ltd. } \\
\text { Takeda Pharmaceutical } \\
\text { Company Limited. } \\
\text { Chugai Pharmaceutical } \\
\text { Co.,Ltd. } \\
\text { Mochida Pharmaceutical } \\
\text { Co.,Ltd. } \\
\text { Nihon Kohden Corp. } \\
\text { Asahi Kasei Pharma Corp. } \\
\text { Terumo Corporation } \\
\text { TESCO Co. Ltd. } \\
\text { Medtronic } \\
\text { Japan Lifeline Co.,Ltd. } \\
\text { Abbott Vascular Japan } \\
\text { Co., Ltd. } \\
\text { St. Jude Medical Japan } \\
\text { Co., Ltd. } \\
\text { Hitachi, Ltd. } \\
\text { Hiara }\end{array}$ & & \\
\hline $\begin{array}{l}\text { Member: } \\
\text { Keiko } \\
\text { Yamauchi- } \\
\text { Takihara }\end{array}$ & & & & & & & $\begin{array}{l}\text { Actelion Pharmaceuticals } \\
\text { Japan Ltd. }\end{array}$ & & \\
\hline $\begin{array}{l}\text { Member: } \\
\text { Koichiro } \\
\text { Tatsumi }\end{array}$ & & & & $\begin{array}{l}\text { Actelion } \\
\text { Pharmaceuticals } \\
\text { Japan Ltd. } \\
\text { Boehringer } \\
\text { Ingelheim } \\
\text { Japan, Inc. }\end{array}$ & & $\begin{array}{l}\text { Teijin Home } \\
\text { Healthcare } \\
\text { Limited. } \\
\text { Ono } \\
\text { Pharmaceutical } \\
\text { Co., Ltd. } \\
\text { Kihara Memorial } \\
\text { Yokohama } \\
\text { Foundation } \\
\text { for the } \\
\text { Advancement } \\
\text { of Life Sciences } \\
\text { D. Western } \\
\text { Therapeutics } \\
\text { Institute, Inc. } \\
\text { Boehringer } \\
\text { Ingelheim } \\
\text { Japan, Inc. }\end{array}$ & $\begin{array}{l}\text { Pfizer Japan Inc. } \\
\text { Boehringer Ingelheim } \\
\text { Japan, Inc. } \\
\text { Astellas Pharma Inc. } \\
\end{array}$ & & \\
\hline
\end{tabular}




\begin{tabular}{|c|c|c|c|c|c|c|c|c|c|}
\hline Author & $\begin{array}{c}\text { Employer/ } \\
\text { leadership } \\
\text { position } \\
\text { (private } \\
\text { company) }\end{array}$ & $\begin{array}{l}\text { Stake- } \\
\text { holder }\end{array}$ & $\begin{array}{l}\text { Patent } \\
\text { royalty }\end{array}$ & Honorarium & $\begin{array}{l}\text { Payment } \\
\text { for } \\
\text { manu- } \\
\text { scripts }\end{array}$ & Research grant & $\begin{array}{c}\text { Scholarship } \\
\text { (educational) } \\
\text { grant/endowed } \\
\text { chair }\end{array}$ & $\begin{array}{l}\text { Other } \\
\text { rewards }\end{array}$ & $\begin{array}{l}\text { Potential } \\
\text { COI of the } \\
\text { marital } \\
\text { partner, } \\
\text { first-degree } \\
\text { family } \\
\text { members, } \\
\text { or those } \\
\text { who share } \\
\text { income and } \\
\text { property }\end{array}$ \\
\hline $\begin{array}{l}\text { Member: } \\
\text { Hiroshi } \\
\text { Date }\end{array}$ & & & & $\begin{array}{l}\text { Johnson \& } \\
\text { Johnson K.K. } \\
\text { Covidien plc }\end{array}$ & & & $\begin{array}{l}\text { Taiho Pharmaceutical } \\
\text { Co., Ltd. }\end{array}$ & & \\
\hline $\begin{array}{l}\text { Member: } \\
\text { Shozaburo } \\
\text { Doi }\end{array}$ & & & & $\begin{array}{l}\text { Actelion } \\
\text { Pharmaceuticals } \\
\text { Japan Ltd. }\end{array}$ & & & JA Ibaraki kouseiren & & \\
\hline $\begin{array}{l}\text { Member: } \\
\text { Masaharu } \\
\text { Nishimura }\end{array}$ & & & & & & & $\begin{array}{l}\text { Actelion Pharmaceuticals } \\
\text { Japan Ltd. }\end{array}$ & & \\
\hline $\begin{array}{l}\text { Member: } \\
\text { Masaru } \\
\text { Hatano }\end{array}$ & & & & $\begin{array}{l}\text { Actelion } \\
\text { Pharmaceuticals } \\
\text { Japan Ltd. } \\
\text { Nippon Shinyaku } \\
\text { Co., Ltd. } \\
\text { Bayer Yakuhin, } \\
\text { Ltd. } \\
\text { Pfizer Japan Inc. }\end{array}$ & & & $\begin{array}{l}\text { GlaxoSmithKline K.K. } \\
\text { Nippon Shinyaku Co., } \\
\text { Ltd. } \\
\text { Bayer Yakuhin, Ltd. }\end{array}$ & & \\
\hline $\begin{array}{l}\text { Member: } \\
\text { Yoshihiro } \\
\text { Fukumoto }\end{array}$ & & & & $\begin{array}{l}\text { Otsuka } \\
\text { Pharmaceutical } \\
\text { Co., Ltd. } \\
\text { Kowa } \\
\text { Pharmaceutical } \\
\text { Co., Ltd. } \\
\text { Daiichi Sankyo } \\
\text { Company, } \\
\text { Limited. } \\
\text { Nippon Shinyaku } \\
\text { Co., Ltd. } \\
\text { Boehringer } \\
\text { Ingelheim } \\
\text { Japan, Inc. } \\
\text { Bayer Yakuhin, } \\
\text { Ltd. } \\
\text { Pfizer Japan Inc. } \\
\end{array}$ & & $\begin{array}{l}\text { Sanwa Kagaku } \\
\text { Kenkyusho } \\
\text { Co., Ltd. }\end{array}$ & $\begin{array}{l}\text { Novartis Pharma K.K. } \\
\text { Japan Lifeline Co.,Ltd. } \\
\text { Bristol-Myers Squibb } \\
\text { Pfizer Japan Inc. } \\
\text { Takeda Pharmaceutical } \\
\text { Company Limited. } \\
\text { Astellas Pharma Inc. } \\
\text { Actelion Pharmaceuticals } \\
\text { Japan Ltd. } \\
\text { MSD K.K. } \\
\text { Teijin Pharma Limited. } \\
\text { Shionogi \& Co. Ltd. }\end{array}$ & & \\
\hline $\begin{array}{l}\text { Member: } \\
\text { Hiromi } \\
\text { Matsubara }\end{array}$ & & & & $\begin{array}{l}\text { Actelion } \\
\text { Pharmaceuticals } \\
\text { Japan Ltd. } \\
\text { Bayer Yakuhin, } \\
\text { Ltd. }\end{array}$ & & & $\begin{array}{l}\text { Nippon Shinyaku Co., } \\
\text { Ltd. }\end{array}$ & & \\
\hline $\begin{array}{l}\text { Member: } \\
\text { Shin-ichi } \\
\text { Momomura }\end{array}$ & & & & $\begin{array}{l}\text { Novartis Pharma } \\
\text { K.K. } \\
\text { Boehringer } \\
\text { Ingelheim } \\
\text { Japan, Inc. } \\
\text { Bayer Yakuhin, } \\
\text { Ltd. } \\
\text { Daiichi Sankyo } \\
\text { Company, } \\
\text { Limited. } \\
\text { Bristol-Myers } \\
\text { Squibb }\end{array}$ & & & Roche Diagnostics K.K. & & \\
\hline $\begin{array}{l}\text { Member: } \\
\text { Norikazu } \\
\text { Yamada }\end{array}$ & & & & $\begin{array}{l}\text { Bayer Yakuhin, } \\
\text { Ltd. } \\
\text { Daiichi Sankyo } \\
\text { Company, } \\
\text { Limited. } \\
\text { Kawasumi } \\
\text { Laboratories. } \\
\text { Inc. } \\
\end{array}$ & & & & & \\
\hline $\begin{array}{l}\text { Member: } \\
\text { Shunji } \\
\text { Yoshida }\end{array}$ & & & & & & & Astellas Pharma Inc. & & \\
\hline $\begin{array}{l}\text { Collaborator: } \\
\text { Kohtaro } \\
\text { Abe }\end{array}$ & & & & $\begin{array}{l}\text { Actelion } \\
\text { Pharmaceuticals } \\
\text { Japan Ltd. }\end{array}$ & & & & & \\
\hline
\end{tabular}




\begin{tabular}{|c|c|c|c|c|c|c|c|c|c|}
\hline Author & $\begin{array}{l}\text { Employer/ } \\
\text { leadership } \\
\text { position } \\
\text { (private } \\
\text { company) }\end{array}$ & $\begin{array}{l}\text { Stake- } \\
\text { holder }\end{array}$ & $\begin{array}{l}\text { Patent } \\
\text { royalty }\end{array}$ & Honorarium & $\begin{array}{l}\text { Payment } \\
\text { for } \\
\text { manu- } \\
\text { scripts }\end{array}$ & Research grant & $\begin{array}{c}\text { Scholarship } \\
\text { (educational) } \\
\text { grant/endowed } \\
\text { chair }\end{array}$ & $\begin{array}{l}\text { Other } \\
\text { rewards }\end{array}$ & $\begin{array}{c}\text { Potential } \\
\text { COI of the } \\
\text { marital } \\
\text { partner, } \\
\text { first-degree } \\
\text { family } \\
\text { members, } \\
\text { or those } \\
\text { who share } \\
\text { income and } \\
\text { property }\end{array}$ \\
\hline $\begin{array}{l}\text { Collaborator: } \\
\text { Takeshi } \\
\text { Ogo }\end{array}$ & & & & $\begin{array}{l}\text { Actelion } \\
\text { Pharmaceuticals } \\
\text { Japan Ltd. } \\
\text { Nippon Shinyaku } \\
\text { Co., Ltd. } \\
\text { Bayer Yakuhin, } \\
\text { Ltd. }\end{array}$ & & & $\begin{array}{l}\text { Actelion Pharmaceuticals } \\
\text { Japan Ltd. }\end{array}$ & & \\
\hline $\begin{array}{l}\text { Collaborator: } \\
\text { Aiko } \\
\text { Ogawa }\end{array}$ & & & & & & & $\begin{array}{l}\text { Nippon Shinyaku Co., } \\
\text { Ltd. }\end{array}$ & & \\
\hline $\begin{array}{l}\text { Collaborator: } \\
\text { Takatoshi } \\
\text { Kasai }\end{array}$ & & & & & & $\begin{array}{l}\text { Sanwa Kagaku } \\
\text { Kenkyusho } \\
\text { Co., Ltd. } \\
\text { Asahi Kasei } \\
\text { Corporation. }\end{array}$ & $\begin{array}{l}\text { Koninklijke Philips N.V. } \\
\text { Fukuda Denshi Co., Ltd. } \\
\text { ResMed }\end{array}$ & & \\
\hline $\begin{array}{l}\text { Collaborator: } \\
\text { Masaharu } \\
\text { Kataoka }\end{array}$ & & & & & & & $\begin{array}{l}\text { Actelion Pharmaceuticals } \\
\text { Japan Ltd. }\end{array}$ & & \\
\hline $\begin{array}{l}\text { Collaborator: } \\
\text { Takashi } \\
\text { Kawakami }\end{array}$ & & & & $\begin{array}{l}\text { Bayer Yakuhin, } \\
\text { Ltd. }\end{array}$ & & & $\begin{array}{l}\text { Actelion Pharmaceuticals } \\
\text { Japan Ltd. }\end{array}$ & & \\
\hline $\begin{array}{l}\text { Collaborator: } \\
\text { Shigetoyo } \\
\text { Kogaki }\end{array}$ & & & & $\begin{array}{l}\text { Actelion } \\
\text { Pharmaceuticals } \\
\text { Japan Ltd. }\end{array}$ & & & & & \\
\hline $\begin{array}{l}\text { Collaborator: } \\
\text { Koichiro } \\
\text { Sugimura }\end{array}$ & & & & $\begin{array}{l}\text { Daiichi Sankyo } \\
\text { Company, } \\
\text { Limited. } \\
\text { Bayer Yakuhin, } \\
\text { Ltd. } \\
\text { Sumitomo } \\
\text { Dainippon } \\
\text { Pharma Co., } \\
\text { Ltd. } \\
\text { Otsuka } \\
\text { Pharmaceutical } \\
\text { Co., Ltd. } \\
\text { Sanofi K.K. }\end{array}$ & & & & & \\
\hline $\begin{array}{l}\text { Collaborator: } \\
\text { Nobuhiro } \\
\text { Tanabe }\end{array}$ & & & & $\begin{array}{l}\text { Actelion } \\
\text { Pharmaceuticals } \\
\text { Japan Ltd. } \\
\text { Nippon Shinyaku } \\
\text { Co., Ltd. } \\
\text { Bayer Yakuhin, } \\
\text { Ltd. } \\
\text { Daiichi Sankyo } \\
\text { Company, } \\
\text { Limited. } \\
\text { Pfizer Japan Inc. }\end{array}$ & & & $\begin{array}{l}\text { Nippon Shinyaku Co., } \\
\text { Ltd. } \\
\text { Actelion Pharmaceuticals } \\
\text { Japan Ltd. }\end{array}$ & & \\
\hline $\begin{array}{l}\text { Collaborator: } \\
\text { Ichizo } \\
\text { Tsujino }\end{array}$ & & & & $\begin{array}{l}\text { Actelion } \\
\text { Pharmaceuticals } \\
\text { Japan Ltd. }\end{array}$ & & & $\begin{array}{l}\text { Actelion Pharmaceuticals } \\
\text { Japan Ltd. }\end{array}$ & & \\
\hline $\begin{array}{l}\text { Collaborator: } \\
\text { Mashio } \\
\text { Nakamura }\end{array}$ & & & & $\begin{array}{l}\text { Daiichi Sankyo } \\
\text { Company, } \\
\text { Limited. }\end{array}$ & & & & & \\
\hline
\end{tabular}

Companies are listed only by name.

The following members and collaborators have no relevant COIs.

Member: Hatsue Ishibashi-Ueda, none

Member: Norihide Fukushima, none

Collaborator: Tomotaka Nakayama, none

Collaborator: Mari Nishizaki, none

Collaborator: Atsushi Yao, none 